volume 3

ISSUE 4

2015 Ottobre

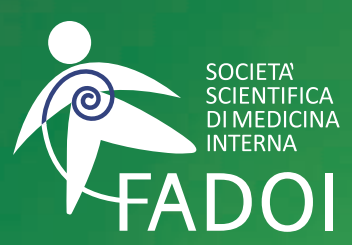

FEDERAZIONE

DELLE ASSOCIAZIONI

DEI DIRIGENTI

OSPEDALIERI

INTERNISTI

Editor in Chief

Roberto Nardi

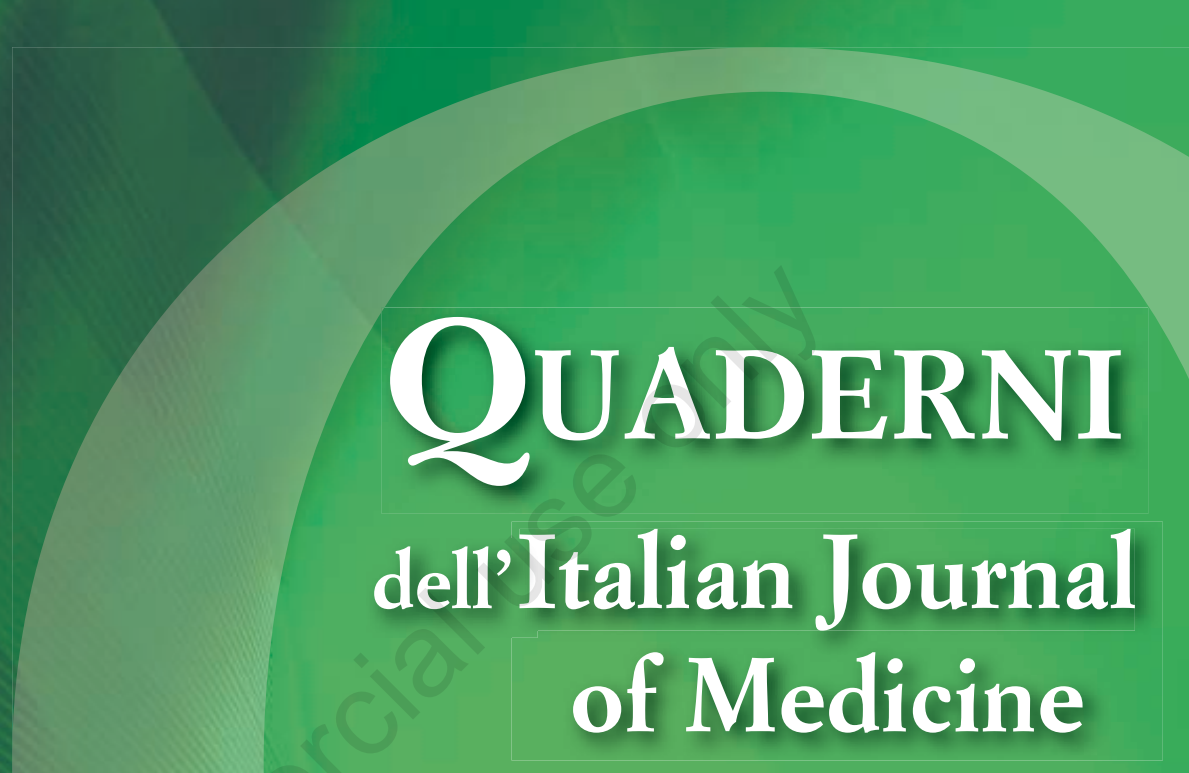

A Journal of Hospital

and Internal Medicine

The official journal of the Federation of Associations of Hospital Doctors on Internal Medicine (FADOI)

\title{
Position paper FADOI sulla prevenzione cardiovascolare nei pazienti complessi a rischio
}

Guest Editors: M. Campanini, R. Nardi, G. Pinna 
Mauro Campanini, Novara, Italy

\section{PRESIDENTE ELETTO}

Andrea Fontanella, Napoli, Italy

\section{PAST PRESIDENT}

Carlo Nozzoli, Firenze, Italy

\section{SEGRETARIO}

Giuseppe Augello, Canicattì (AG), Italy

\section{TESORIERE}

Dario Manfellotto, Roma, Italy

DIRETTORE RIVISTA

ITALIAN JOURNAL OF MEDICINE

Roberto Nardi, Bologna, Italy
RESPONSABILE RAPPORTI SOCIETÀ SCIENTIFICHE E ISTITUZIONI

Antonino Mazzone, Legnano (MI), Italy

RESPONSABILE SISTEMA GESTIONE QUALITÀ

Franco Berti, Roma, Italy

RESPONSABILE SITO NAZIONALE

Francesco Cipollini, Ascoli Piceno, Italy

DELEGATO FADOI ITALIAN STROKE ORGANIZATION

Michele Stornello, Siracusa, Italy

COMMISSIONE FADOI GIOVANI

Paola Gnerre, Savona, Italy

\section{Fondazione FADOI - Organigramma}

PRESIDENTE

Giorgio Vescovo, Padova, Italy

\section{COORDINATORE}

Domenico Panuccio, Bologna, Italy

\section{DIRETTORE SCIENTIFICO}

Gualberto Gussoni, Milano, Italy

DIRETTORE DIPARTIMENTO PER LA FORMAZIONE ED AGGIORNAMENTO

Mauro Silingardi, Guastalla (RE), Italy
DIRETTORE DIPARTIMENTO PER LA RICERCA CLINICA

Giancarlo Agnelli, Perugia, Italy

\section{CONSIGLIO DI AMMINISTRAZIONE}

Consigliere

Consigliere

Consigliere

Consigliere

Consigliere

Consigliere

Consigliere

Consigliere

Consigliere
Paolo Arullani, Roma, Italy

Davide Croce, Castellanza (VA), Italy

Francesco D'Amore, Roma, Italy

Salvatore Di Rosa, Palermo, Italy

Sandro Fontana, Biella, Italy

Ranieri Guerra, Roma, Italy

Ido Iori, Reggio Emilia, Italy

Giovanni Mathieu, Pinerolo (TO), Italy

Cecilia Politi, Isernia, Italy 


\section{QUADERNI - Italian Journal of Medicine}

Position paper FADOI (Federazione delle Associazioni dei Dirigenti Ospedalieri Internisti) sulla prevenzione cardiovascolare nei pazienti complessi a rischio .

M. Campanini, G. Pinna, R. Nardi

La prevenzione secondaria nella cardiopatia ischemica

D. Panuccio, P. Verdecchia

La prevenzione cardiovascolare nello scompenso cardiaco

R. Cavaliere, F. Gallucci, G. Mathieu

Prevenzione secondaria degli eventi cerebrovascolari aterotrombotici

M. Paciaroni, R. Frediani, M. Stornello, G. Agnelli

Prevenzione cardiovascolare secondaria e nefropatia

D. Manfellotto, G. Vescovo

Arteriopatia obliterante periferica cronica degli arti inferiori ....

F. Dentali, A. Mazzone

La prevenzione cardiovascolare secondaria nel paziente con diabete mellito 


\title{
Position paper FADOI (Federazione delle Associazioni dei Dirigenti Ospedalieri Internisti) sulla prevenzione cardiovascolare nei pazienti complessi a rischio
}

\author{
Mauro Campanini, ${ }^{1}$ Giuliano Pinna, ${ }^{2}$ Roberto $\mathrm{Nardi}^{3}$ \\ ${ }^{1}$ Medicina Interna 2, AOU Maggiore della Carità, Novara; ${ }^{2}$ Medicina Interna, Asti; ${ }^{3}$ Medicina Interna, Bologna, Italy
}

\section{Presentazione}

Gli obiettivi della medicina sono quelli di promuovere o preservare la salute, ovvero di ripristinarla quando compromessa, per ridurre al minimo la sofferenza e la disabilità dei nostri pazienti. Le malattie cardiovascolari (CV) rappresentano la prima causa di decessi in Europa e nel mondo. Anche in Italia costituiscono uno dei maggiori problemi di salute pubblica, con imponenti implicazioni sulle risorse sanitarie e economiche, causa rilevante di mortalità, morbilità ed invalidità. Le più frequenti patologie CV comprese fra le cosiddette chronic noncommunicable diseases sono, in particolare, le cardiopatie ischemiche (infarto acuto del miocardio e angina pectoris), le malattie cerebrovascolari (ictus ischemico ed emorragico), l'arteriopatia periferica e la malattia nefro-vascolare cronica, cui si aggiungono alcune condizioni morbose gravate da elevata prevalenza ed incidenza quali il diabete mellito, le malattie metaboliche e lo scompenso cardiaco. Da uno studio di confronto effettuato da OEC/HES (Osservatorio Epidemiologico Cardio-vascolare/ Health Epidemiologic Study) emerge che nel corso del decennio 1998-2008 nella popolazione di età compresa tra i 35 e i 69 anni il rischio cardiovascolare globale, ovvero la probabilità di andare incontro $\mathrm{a}$ un primo evento cardiovascolare maggiore nei successivi

Corrispondente: Roberto Nardi, via C. Pavese 16/2, 40141 Bologna, Italy.

Tel.: +39.335.8291342.

E-mail: nardidoc48@gmail.com

Articolo pubblicato secondo la Creative Commons Attribution NonCommercial 3.0 License (CC BY-NC 3.0).

CCopyright M. Campanini et al., 2015

Licensee PAGEPress, Italy

QUADERNI - Italian Journal of Medicine 2015; 3:309-318
Neanche una mela al giorno leva il medico di torno? ${ }^{1}$

10 anni, stimato con la funzione del Progetto Cuore, si è ridotto nelle donne, passando dal 3,2 al 2,7\%, risultando invece invariato, intorno all' $8 \%$ circa, negli uomini. ${ }^{2}$ Purtroppo pochi dati sono disponibili negli anziani, anche se è stato recentemente evidenziato che una terapia evidence based nella prevenzione $\mathrm{CV}$ secondaria è utile nelle fasce di età più avanzate, ad esempio, dopo un infarto miocardico acuto. ${ }^{3}$

\section{Rapporto rischio assoluto/rischio relativo}

Il rapporto tra rischio assoluto e rischio relativo (RR) è illustrato dalla seguente equazione:

$\mathrm{RR}=\frac{\text { Incidenza negli esposti }}{\text { Incidenza nei non esposti }}$

dove:

rischio assoluto $=$ probabilità, osservata o calcolata, $\mathrm{di}$ un evento cardiovascolare, fatale o non fatale, in un soggetto o in una popolazione in studio;

rischio relativo $=$ il rapporto fra il rischio di un evento cardiovascolare, fatale o non fatale, negli esposti rispetto ai non esposti.

\section{Come intervenire sui fattori di rischio cardiovascolare?}

Le strategie rivolte alla correzione dei fattori di rischio per una malattia all'interno di una popolazione sono fondamentalmente tre: i) rivolte a tutta la popolazione (strategia di comunità); ii) rivolte a singoli soggetti caratterizzati da alti livelli di un singolo fattore di rischio; iii) rivolte a singoli soggetti caratterizzati da alti livelli di rischio complessivo (vale a dire da un mix di diversi livelli d'esposizione a diversi fattori di rischio) (Tabella 1) ${ }^{4-7}$

Si conferma la necessità di una strategia nella gestione delle situazioni di rischio, sulla base dei principi fondanti della prevenzione (Tabella 2). ${ }^{8-11}$ 
In un'epoca molto attenta all'impiego appropriato delle risorse, si è aggiunto agli elementi riportati in Tabella 2 l'ulteriore concetto di prevenzione quaternaria ${ }^{10,11}$ ovvero di tutte le misure adottate per prevenire, ridurre e/o alleviare i danni causati da attività sanitarie, in grado, di fatto, di produrre non solamente benefici, ma anche danni, ad esempio per attività mediche inutili o con l'impiego inappropriato di misure di prevenzione di non comprovata efficacia. In sostanza la protezione rivolta ai nostri pazienti dovrebbe

Tabella 1. Le possibili strategie finalizzate alla correzione dei fattori di rischio.

\begin{tabular}{|c|c|c|}
\hline \multicolumn{2}{|l|}{ Strategia } & Intervento \\
\hline \multicolumn{2}{|c|}{ Population based o strategia di comunità } & $\begin{array}{l}\text { Agire su tutti i soggetti indipendentemente dai livelli di esposizione al fattore di rischio } \\
\text { La strategia di comunità segue l'approccio di Geoffrey Rose, in base al postulato che gli } \\
\text { interventi sulla popolazione, pur se con un piccolo beneficio individuale, possono avere } \\
\text { un grande effetto collettivo, particolarmente quando il rischio è diffuso tra tutta la } \\
\text { popolazione }\end{array}$ \\
\hline \multirow[t]{2}{*}{ Patient based } & Single risk factor strategy & Agire solo sui soggetti con elevati livelli di esposizione ad un determinato fattore di rischio \\
\hline & High baseline risk strategy & $\begin{array}{l}\text { Agire solo sui soggetti caratterizzati da esposizione a diversi fattori di rischio, con } \\
\text { conseguenti alti livelli di rischio complessivo }{ }^{6}\end{array}$ \\
\hline
\end{tabular}

Modificata da Giustini e Battaggia.?

Tabella 2. Differenze concettuali tradizionali tra prevenzione primordiale, primaria, secondaria e terziaria.

\begin{tabular}{|c|c|c|c|}
\hline Livello di prevenzione $e^{9-11}$ & Definizione & Tipo di risposta & $\begin{array}{l}\text { Possibili opportunità su cui intervenire } \\
\text { in tema di rischio } \mathrm{CV}\end{array}$ \\
\hline Prevenzione primordiale & $\begin{array}{l}\text { Prevenzione dei fattori } \\
\text { di rischio nel soggetto che } \\
\text { non ha né fattori di rischio, } \\
\text { né danni d'organo, né eventi } \\
\text { cardiovascolari }\end{array}$ & $\begin{array}{l}\text { Presuppone azioni e misure } \\
\text { di promozione della salute } \\
\text { finalizzate ad inibire l'emergere } \\
\text { di possibili fattori di rischio CV, } \\
\text { nel contesto ambientale, } \\
\text { economico e sociale, con } \\
\text { proposizione di modelli culturali } \\
\text { di vita comportamentali adeguati } \\
\text { Molti problemi di salute degli } \\
\text { adulti (come obesità ed ipertensione) } \\
\text { hanno le loro prime origini } \\
\text { nell'infanzia, quando si formano } \\
\text { gli stili di vita, per esempio, riguardo } \\
\text { il fumo, le abitudini alimentari, } \\
\text { l'esercizio fisico }\end{array}$ & $\begin{array}{l}\text { Predisposizione (costituzione) famigliare } \\
\text { Fattori psico-sociali } \\
\text { Peso ideale/peso forma } \\
\text { Alimentazione salutare } \\
\text { Limitazione dell'alcool } \\
\text { Attività fisica }\end{array}$ \\
\hline Prevenzione primaria & $\begin{array}{l}\text { Prevenzione dei fattori di } \\
\text { rischio nel soggetto che non } \\
\text { ha né fattori di rischio } \\
\text { né danni d'organo, né eventi } \\
\text { cardiovascolari }\end{array}$ & $\begin{array}{l}\text { Qualsiasi azione intrapresa prima } \\
\text { dell'inizio della malattia finalizzata } \\
\text { ad evitare che la malattia insorga } \\
\text { (prevention of occurrence), } \\
\text { eliminando i fattori causali delle } \\
\text { malattie e trattando gli stati di rischio } \\
\text { (ad esempio con cambiamenti } \\
\text { comportamentali), o aumentando la } \\
\text { resistenza dell'organismo, ad esempio } \\
\text { con il ricorso alla vaccinazione o } \\
\text { attraverso modifiche ambientali }\end{array}$ & $\begin{array}{l}\text { Tutti gli elementi considerati nella } \\
\text { prevenzione primordiale } \\
\text { Sospensione del fumo } \\
\text { Limitazione dell'alcool } \\
\text { Diagnosi e gestione del diabete mellito, } \\
\text { dell'ipertensione arteriosa, } \\
\text { delle dislipidemie }\end{array}$ \\
\hline Prevenzione secondaria & $\begin{array}{l}\text { Prevenzione di ulteriori } \\
\text { eventi cardiovascolari nel } \\
\text { paziente che ha già avuto } \\
\text { almeno un evento } \\
\text { cardiovascolare }\end{array}$ & $\begin{array}{l}\text { Si focalizza sui pazienti che hanno } \\
\text { già avuto una manifestazione di } \\
\text { malattia e che rischiano un } \\
\text { deterioramento clinico e/o ulteriori } \\
\text { complicazioni }\end{array}$ & $\begin{array}{l}\text { Tutti gli elementi considerati nella } \\
\text { prevenzione primaria } \\
\text { Follow-up e controllo } \\
\text { Farmaci }\end{array}$ \\
\hline Prevenzione terziaria & $\begin{array}{l}\text { Prevenzione delle } \\
\text { complicanze e/o delle } \\
\text { recidive di un evento }\end{array}$ & $\begin{array}{l}\text { Presuppone tutte le misure disponibili } \\
\text { per ridurre o limitare qualsiasi } \\
\text { menomazione o disabilità, nonché } \\
\text { promuovere la gestione dei pazienti } \\
\text { già colpiti da malattia }\end{array}$ & $\begin{array}{l}\text { Tutti gli elementi considerati nella } \\
\text { prevenzione secondaria } \\
\text { Follow up e controllo } \\
\text { Riabilitazione }\end{array}$ \\
\hline
\end{tabular}

CV, cardiovascolare. Fonte Benjamin et al., 2002. ${ }^{8}$ 
considerare anche i pazienti a rischio di diagnosi inappropriata o di sovra-trattamento, allo scopo di proporre, al contrario, trattamenti realmente utili.

\section{Ha ancora senso oggi parlare di prevenzione primaria e secondaria?}

La differenziazione fra prevenzione primordiale, primaria (evitare l'insorgenza della malattia nei sani) e prevenzione secondaria e terziaria (evitare la progressione della patologia e le complicanze negli ammalati) non è affatto un concetto scontato o semplice. Essa richiede una definizione del concetto di malattia tale da consentire di distinguere tra soggetti sani e malati. Le categorie indicate sono arbitrarie e vengono utilizzate al fine di sistematizzare una materia complessa per l'organizzazione del sistema sanitario, ma non possono essere escluse possibili sovrapposizioni tra l'una e l'altra. ${ }^{12}$ Il concetto di prevenzione primaria e secondaria non si correla al riconoscimento che l'aterosclerosi è un continuo processo in evoluzione: il tema posto del danno d'organo subclinico (e non l'evento) come spartiacque dovrebbe per l'appunto essere finalizzato a tutelare precocemente il paziente in cui vengono rilevati elementi di rischio. La malattia subclinica è un tema di grande rilevanza epidemiologica anche per $1^{1}$ Internista, ${ }^{13}$ che deve avere la capacità di ricercare le patologie ad iceberg o sottese, in base ad una valutazione globale. L'introduzione di diverse tecniche diagnostiche ha consentito di porre diagnosi di malattia anche in assenza di sintomatologia manifesta. ${ }^{14}$ Il discrimine fra tali situazioni era tradizionalmente definito dalla presenza o assenza di eventi cardiovascolari maggiori clinicamente manifesti con sintomi espliciti (dolore toracico, sintomi neurologici focali, claudicatio, ecc.): oggi, grazie alla diagnostica strumentale anche non invasiva, è possibile rilevare danni d'organo e/o situazioni patologiche sub-cliniche, spesso ancora asintomatiche, che vanno dalla presenza di ispessimento medio-intimale, al rilievo di alterazione del rapporto pressorio caviglia/braccio, alla presenza di calcificazioni coronariche e/o di placche aterosclerotiche, alla presenza di microalbuminuria. ${ }^{15}$ Proprio per tali motivi, le priorità di valutazione vengono proposte dalla letteratura secondo quattro livelli: pazienti con malattia diagnosticata, individui asintomatici ad alto rischio di mortalità $\mathrm{CV}$, parenti di primo grado di pazienti con malattia $\mathrm{CV}$ prematura e altri pazienti incontrati nella pratica clinica di routine. ${ }^{16}$ Da qui la necessità di interventi di prevenzione basati sulla valutazione individuale del rischio $\mathrm{CV}$ di ogni singolo paziente, quindi con interventi sui molteplici fattori di rischio e col trattamento delle condizioni cliniche associate: la strategia dovrebbe essere in sostanza quella di trattare il livello di rischio, piuttosto che il fattore di rischio. In altri termini, per ridurre il rischio $\mathrm{CV}$ globale è necessario identificare e trattare tutti i fattori di rischio coesistenti nello stesso individuo, proponendo interventi multifattoriali, personalizzati per ogni paziente e definendo obiettivi espliciti. ${ }^{17}$ In Medicina Interna la gestione dei pazienti ad elevato rischio $\mathrm{CV}$ o con malattia $\mathrm{CV}$ accertata è resa particolarmente problematica in ragione della complessità dei pazienti, che condiziona fortemente il processo decisionale del medico. Secondo un recente studio cross-sectional condotto in soggetti ultra 45enni con cardiopatia coronarica sono in causa diversi elementi, quali: i) la coesistenza di altre malattie croniche: artrosi (nel 56,7\% dei soggetti esaminati), scompenso cardiaco (nel 29\%), COPD e diabete mellito (nel 25\% dei soggetti), stroke (nel 13,8\%); ii) alterazione dei dati di laboratorio e presenza di fattori indicativi di complessità clinica: ridotta filtrazione glomerulare (nel 24,4\% dei soggetti), anemia (nel $10,1 \%$ ), alterazione delle transaminasi (nel 5,9\%), assunzione di più di 4 farmaci (nel 54,5\% dei casi), incontinenza urinaria (nel 48,6\%), vertigini o cadute (nel 34,8\%), terapia anticoagulante orale (nel 10,2\%); iii) fattori generali indicativi di un alterato stato di salute: deficit cognitivo (presente nel $29,9 \%$ dei soggetti esaminati), difficoltà di movimento $(40,4 \%$ dei casi), difetto del visus o dell'udito (nel 16,7 e nel 17,9\% dei casi, rispettivamente).$^{18} \mathrm{Nel}$ paziente complesso le comorbilità comportano interrelazioni rilevanti tra fattori di rischio comuni, il cui controllo può facilitarne la gestione. Un esempio eclatante è costituito dalla broncopneumopatia cronica ostruttiva (BPCO), in cui sono dimostrate un'aumentata morbilità e mortalità CV. ${ }^{19,20}$ Vanno considerate le possibili diseguaglianze nell'accesso alle cure (uno strumento introdotto in merito è quello del Townsend deprivation score), ${ }^{21}$ le associazioni e i clusters di malattia, che possono influenzare la prognosi dei pazienti, ${ }^{22}$ come, ad esempio, nel caso di un'anamnesi contrassegnata da episodi di fibrillazione atriale. Le comorbilità possono comportare un aumentato rischio cardiovascolare non solo per i possibili fattori di rischio comuni alle diverse malattie, come il fumo, l'obesità, l'ipertensione e l'abuso di alcol, ma anche per la fisiopatologia stessa delle malattie, quali, per es., l'HIV, indipendentemente dal trattamento (l'infezione, di per sé, amplifica meccanismi addizionali pro-aterogenici ${ }^{23} \mathrm{o}$ in relazione al trattamento, ad esempio in corso di terapia antiretrovirale (highly active antiretroviral therapy) ${ }^{24} \mathrm{o}$ con corticosteroidi, acitretina e ciclosporina. Similmente, anche l'infezione da virus dell'epatite C può comportare un aumentato rischio $\mathrm{CV}^{25,26}$ Non va infine sottovalutato il ruolo dell'infiammazione, delle citochine pro-infiammatorie e della leptina ${ }^{27}$ nella patogenesi dell'aterosclerosi, che accomuna tale patologia a diverse altre condizioni apparentemente non 
correlate tra loro, ma che condividono meccanismi patogenetici analoghi, tanto da delineare un ambito nosologico specifico [i cosiddetti immune mediated inflammatory disorders (IMIDs)], che nella popolazione generale possono raggiungere una prevalenza fino al 5-7\%. Lo spettro delle malattie croniche IMID correlate è molto ampio: allergia, asma, malattia di Beçhet, BPCO, malattia di Crohn, rettocolite ulcerosa, sclerosi multipla, psoriasi, artrite reumatoide, spondiloartropatie, sarcoidosi, uveite, vasculiti, ecc. ${ }^{28}$ L'Internista dovrebbe essere in sostanza in grado di attuare il case finding dei pazienti a rischio $\mathrm{CV}^{29}$

\section{Le società scientifiche di cardiologia e le iniziative di FADOI}

L'American Heart Association (AHA), l'American College of Cardiology (ACC), l'American College of Chest Physicians e la Canadian Cardiovascular Society hanno recentemente enfatizzato l'attenzione sulla prevenzione delle malattie cardiovascolari, affrontando il tema dal punto di vista dello stile di vita, della presenza di sovrappeso e/o di obesità e di ipercolesterolemia negli adulti, nonché sull'importanza della terapia antitrombotica. A tali valutazioni si sono aggiunte le linee guida della European Atherosclerosis Society (EAS) e dalla European Society of Cardiology (ESC), con le raccomandazioni emergenti sull'importanza della stratificazione del rischio CV e della riduzione del colesterolo con lipoproteine a bassa densità (c-LDL) nella prevenzione primaria e secondaria. ${ }^{30-36}$ In particolare sono state individuate quattro categorie di soggetti a rischio, meritevoli di specifica attenzione da parte dei medici ai fini della prevenzione $\mathrm{CV}:{ }^{37} \mathrm{i}$ ) soggetti con malattia aterosclerotica cardiovascolare clinicamente manifesta; ii) soggetti con livelli di colesterolo LDL >190 mg/dL e con ipercolesterolemia familiare; iii) i diabetici di età compresa tra 40-75 anni, con livelli di colesterolo LDL tra i 70 e $189 \mathrm{mg} / \mathrm{dL}$ e senza evidenza di malattia cardiovascolare aterosclerotica; iv) soggetti senza evidenza di malattia cardiovascolare o diabete, ma che hanno livelli di colesterolo LDL tra i 70 e $189 \mathrm{mg} / \mathrm{dL}$ e un rischio a 10 anni di malattia cardiovascolare aterosclerotica $>7,5 \%$.

Da tempo la Federazione delle Associazioni dei Dirigenti Ospedalieri Internisti (FADOI) si occupa di rischio cardiovascolare. ${ }^{38,39}$ In due studi (FAPOI-1 del 1996 e FAPOI-2 del 1999) nell'analisi di confronto delle modalità di trattamento di pazienti a rischio $\mathrm{CV}$ dimessi da reparti di Medicina Interna, rispettivamente 6450 (235 reparti) e 8133 pazienti (345 reparti) è emersa una maggiore propensione all'impiego di statine, ma con ancora evidente sottotrattamento di alcune situazioni di rischio, specialmente dell'ipertensione arteriosa, particolarmente nel sesso femminile. ${ }^{40}$ Nello studio NIMEC (National Internal Medicine Equivalent/Complex C-V-@Risk study), ${ }^{41}$ effettuato su 902 soggetti reclutati nel rispetto dei criteri di inclusione ed esclusione $[457 \mathrm{M}, 445 \mathrm{~F}$, età media 72 anni, indice di massa corporea $26,7 \mathrm{~kg} / \mathrm{m}^{2}$ (range: 26,3 $\left.\left.27,1 \mathrm{~kg} / \mathrm{m}^{2}\right)\right]$ su 1316 pazienti totali ricoverati in reparti di Medicina Interna, la prevalenza di sindrome metabolica (SM) secondo i criteri dell' Adult Treatment Panel III (ATP III) è risultata pari al 45,3\% [intervallo di confidenza (IC) 95\%: 41,6-49,1]. Secondo i criteri dell'International Diabetes Federation (IDF) ${ }^{42}$ invece, la prevalenza di SM è risultata pari al 38,6\% (IC 95\%: 34,9-42,3). Stratificando per assenza/presenza di sindrome metabolica secondo ATP III, è risultata una differenza statisticamente significativa relativamente a diverse variabili. Secondo i criteri ATP III, i valori medi di sodiemia, potassiemia e fosfatasi alcalina sono risultati inferiori nel gruppo sindrome metabolica, mentre aspartato aminotransferasi, alanina aminotransferasi (ALT) ed emoglobina sono risultati significativamente superiori. La proporzione di ex fumatori è risultata significativamente superiore nel gruppo sindrome metabolica così come il numero di anni di fumo e il numero di sigarette die. Tra le altre differenze riscontrate veniva sottolineata una maggiore frequenza di fibrillazione atriale parossistica, BPCO (bronco-pneumopatia cronica ostruttiva), insufficienza renale cronica, sincope, angina instabile, vasculopatia periferica, patologie valvolari di grado medio/severo e una frequenza maggiore di placche ateromasiche all'eco-color-Doppler dei tronchi sovra-aortici. L'utilizzo dei criteri IDF confermava, nei soggetti con sindrome metabolica, più alti livelli di ALT ed emoglobina, di vasculopatia periferica e di placche ateromasiche (rilevate all'eco Doppler periferico e dei tronchi sovra-aortici), ma con una maggiore frequenza di scompenso cardiaco, oltre che di fibrillazione atriale parossistica, BPCO, insufficienza renale cronica, sincope e angina instabile. ${ }^{41}$ Dall'analisi epidemiologica congiunta effettuata nel 2015 da FADOI-SIMI (Società Italiana di Medicina Interna) a livello territoriale in Italia su 1877 persone nel 2015, intitolata un metro per la vita, considerando la misura della circonferenza vita, pressione sanguigna e livelli di zucchero nel sangue, è stata evidenziata una prevalenza della SM nel 17,4\% degli uomini e nell' $11,4 \%$ delle femmine. ${ }^{43}$

\section{Nella valutazione del rischio cardiovascolare del paziente internistico è necessario un approccio globale}

Alla luce di tali elementi e nel concetto dell'approccio globale al paziente internistico, riteniamo che nei pazienti complessi, in cui il rapporto rischio/beneficio degli interventi preventivoterapeutici può essere influenzato dalle comorbilità 
concorrenti e dai molteplici bisogni assistenziali, la stratificazione del rischio debba essere migliorata ulteriormente, in alcuni casi con cure più aggressive su pazienti specifici, anche se asintomatici, particolarmente quando siano presenti comorbilità che assumono la configurazione di equivalenti di rischio cardio-vascolare, considerando la reale età vascolare dei pazienti. ${ }^{44}$ Il National Cholesterol Education Program (NCEP) Expert Panel on Detection, Evaluation, and Treatment of High Blood Cholesterol in Adults (ATP III) aveva individuato nel 2004 come equivalenti di rischio $\mathrm{CV}$ alcune manifestazioni cliniche di malattia aterosclerotica: la malattia vascolare periferica, l'aneurisma dell'aorta addominale, la malattia delle arterie carotidi sintomatica, il diabete mellito e tutti i fattori di rischio multiplo che conferiscono un rischio a 10 anni di malattia cardiovascolare superiore al $20 \%$ secondo il Framingham risk score. ${ }^{40,45}$ L'ATP III aveva altresì già identificato le principali caratteristiche della sindrome metabolica: obesità addominale, dislipidemia aterogenica, ipertensione arteriosa, insulinoresistenza \pm intolleranza al glucosio. Aveva anche proposto di inserire uno stato proinfiammatorio e uno stato protrombotico, ma non era riuscita a trovare sufficienti evidenze per proporli di routine. Uno stato proinfiammatorio (riconoscibile clinicamente con l'aumento della proteina C-reattiva) ed uno stato protrombotico (caratterizzato dall'aumento del PAI-1inibitore-1 dell'attivatore del plasminogeno e del fibrinogeno $)^{46}$ sono spesso presenti nella sindrome metabolica e possono essere responsabili non solamente di un aumentato rischio $\mathrm{CV}$, ma anche in grado di sottendere altre condizioni, quali l'obesità sarcopenica, ${ }^{41,47}$ la sindrome dell'ovaio policistico, la steatosi epatica, la litiasi biliare colesterinica, l'asma e alcune forme di cancro. A queste condizioni patologiche potrebbero aggiungersi anche la presenza in anamnesi fisiologica di una nascita pretermine con un basso peso (low birthweight) espressione di un fenotipo parsimonioso, ovvero di un thrifty phenotype ${ }^{48}$ quale fattore di rischio di obesità, sindrome metabolica, insulino-resistenza, diabete di tipo 2, ipertensione e variabilità pressoria in età adulta ${ }^{49,50} \mathrm{o}$, tra le abitudini voluttuarie, oltre che il tabagismo, la propensione/attitudine alcoolica. Nella valutazione complessiva del rischio $\mathrm{CV}$ dovrebbero essere considerate le malattie coesistenti e recenti, ad esempio la presenza (anche sub-clinica) di fibrillazione atriale, di carotidopatia asintomatica, di depressione e/o di disfunzione cognitiva e/o di demenza su base vascolare, d'insufficienza renale anche misconosciuta - da malattia renale cronica, ${ }^{51,52}$ di microalbuminuria, di una sindrome delle apnee ostruttive del sonno ${ }^{53} \mathrm{e}$ di disfunzione erettile,$^{54}$ tutti questi fattori emergenti di rischio cardiovascolare, nonché una precedente ospedalizzazione per polmonite. ${ }^{55}$ Controverso, anche riguardo ai costi/benefici di un dosaggio laboratoristico, rimane il ruolo del deficit di testosterone $e^{56,57}$ e, più recentemente, del deficit di vitamina D. ${ }^{58,59}$ Non bisogna dimenticare, infine, altri aspetti quasi del tutto ignorati, come ad esempio, la parodontopatia con conseguente edentulia precoce, condizioni correlate ad aumentato rischio $\mathrm{CV}^{60,61}$ In sostanza, la valutazione clinica globale, insieme alla considerazione delle comorbilità associate, oltre che di alcune valutazioni antropometriche e di pochi iniziali esami di laboratorio (cui ovviamente possono aggiungersi accertamenti eco-ultrasonografici per indagare lo stato delle arterie) è fondamentale. Ciò a conferma della peculiarità dell'internista di saper dirimere - con una visione sistemica - $\mathrm{i}$ casi di rischio $\mathrm{CV}$ elevato, meritevoli di prevenzione e trattamento (Figura 1). ${ }^{62}$

Una volta individuato il paziente portatore di una malattia aterosclerotica si rende necessaria una prevenzione secondaria, per la quale è richiesto un giudizio clinico complessivo. Nella Figura $2^{63}$ viene schematizzato un algoritmo per ridurre gli eventi cardiovascolari secondari in Medicina Interna.

\section{Quesiti posti agli esperti}

I quesiti posti ai diversi Esperti ai fini della prevenzione per ciascuna singola patologia sono i seguenti: i) Quali modalità di valutazione del rischio e di stratificazione prognostica (anche rispetto alle comorbilità esistenti)? ii) Quali azioni da implementare, ritagliate sul singolo paziente?

\section{Conclusioni e questioni aperte}

La prevenzione rappresenta un tema di grande attualità, da cui nessun sistema sanitario moderno può prescindere. ${ }^{64,65} \mathrm{La}$ discussione sul concetto di prevenzione $\mathrm{CV}$ è molto ampia $\mathrm{e}$ dura da molto tempo: in tale contesto la ricerca non si è mai fermata. Ad esempio, il ruolo della metformina ${ }^{66,67}$ e degli omega-3 in tale ambito è ancora in discussione. ${ }^{68}$ Per raggiungere gli obiettivi terapeutici correlati alla riduzione del colesterolo LDL nella prevenzione $\mathrm{CV}$ con statine sono in causa diversi problemi, quali la corretta conoscenza delle linee guida da parte dei medici; l'uso di statine più efficaci in base alla loro efficacia alta, moderata o bassa intensità nel ridurre c-LDL, anche aumentando dosaggi di statine; il ricorso a strategie di associazione ed una maggiore attenzione alla persistenza in trattamento da parte dei pazienti. Anche recentemente è stata confermata l'utilità incrementale nella riduzione del cLDL dell'aggiunta di ezetimibe alla terapia con statine nei pazienti con precedente sindrome coronarica acuta, 
particolarmente nei diabetici. ${ }^{69,70}$ Oggi si stagliano ulteriori opzioni terapeutiche, nei soggetti a rischio CV molto elevato, basate sull'impiego degli anticorpi monoclonali anti-PCSK9, su cui dovranno essere effettuate ulteriori valutazioni, alla luce delle evidenze disponibili $^{71,72}$ o la terapia con oligonucletidi antisense. ${ }^{73}$ La classificazione schematica dei diversi tipi di prevenzione, così come poste dalla letteratura, pone evidenti limiti che mal si adattano al paziente complesso internistico. In una logica di evidence based medicine dovremmo fare riferimento alle linee guida (LG) disponibili, in base a studi rigorosi condotti su pazienti del mondo reale. Purtroppo la qualità evidence based delle LG è ben lungi dall'essere ottimale, anche quando proposte da Società scientifiche di tutto rispetto. L'AHA e l'ACC avevano già documentato il fatto che la proporzione delle raccomandazioni sviluppate su livelli più bassi di evidenza o basate sul parere di esperti, senza prove conclusive, è in progressiva crescita. ${ }^{74}$ Le LG si basano spesso su studi effettuati in pazienti ideali, a conferma del postulato che le evidenze per l'implementazione delle LG in circostanze complesse sono inadeguate, proprio per mancanza di prove. L'entusiasmo per le LG deve essere sempre temperato con la consapevolezza che esse possono essere fallibili. Ad esempio, anche se recentemente si è iniziato a considerare il problema della comorbilità, sono poche le linee guida che considerano la possibilità che alcuni pazienti possano essere affetti da altre malattie concomitanti, trattate con farmaci ulteriori, con rischio di interazioni farmacologiche e di reazioni avverse, ${ }^{75,76}$ come, ad esempio, in caso di terapia antipertensiva multipla. ${ }^{77}$ In altri termini, è sempre necessario un attento giudizio del medico sul singolo caso, prima di applicare le indicazioni generali delle LG. $^{78,79}$ Ai fini di una valutazione multidimensionale del rischio CV nel paziente complesso non è sufficiente una high baseline risk strategy: dovremmo dimensionare i nostri parametri di valutazione al mondo reale, secondo una strategia di valutazione multidimensionale del rischio cardiovascolare elevato (high multidimensional CV complexity assessment risk strategy), in relazione ad un approccio antropologico alla complessità delle persone. ${ }^{80}$ In sostanza gli internisti ospedalieri sono chiamati ad esercitare un ruolo proattivo di esperto su ciascun singolo paziente complesso, anche nella prevenzione cardio-vascolare. ${ }^{81}$ È necessario individuare i livelli di rischio nel singolo

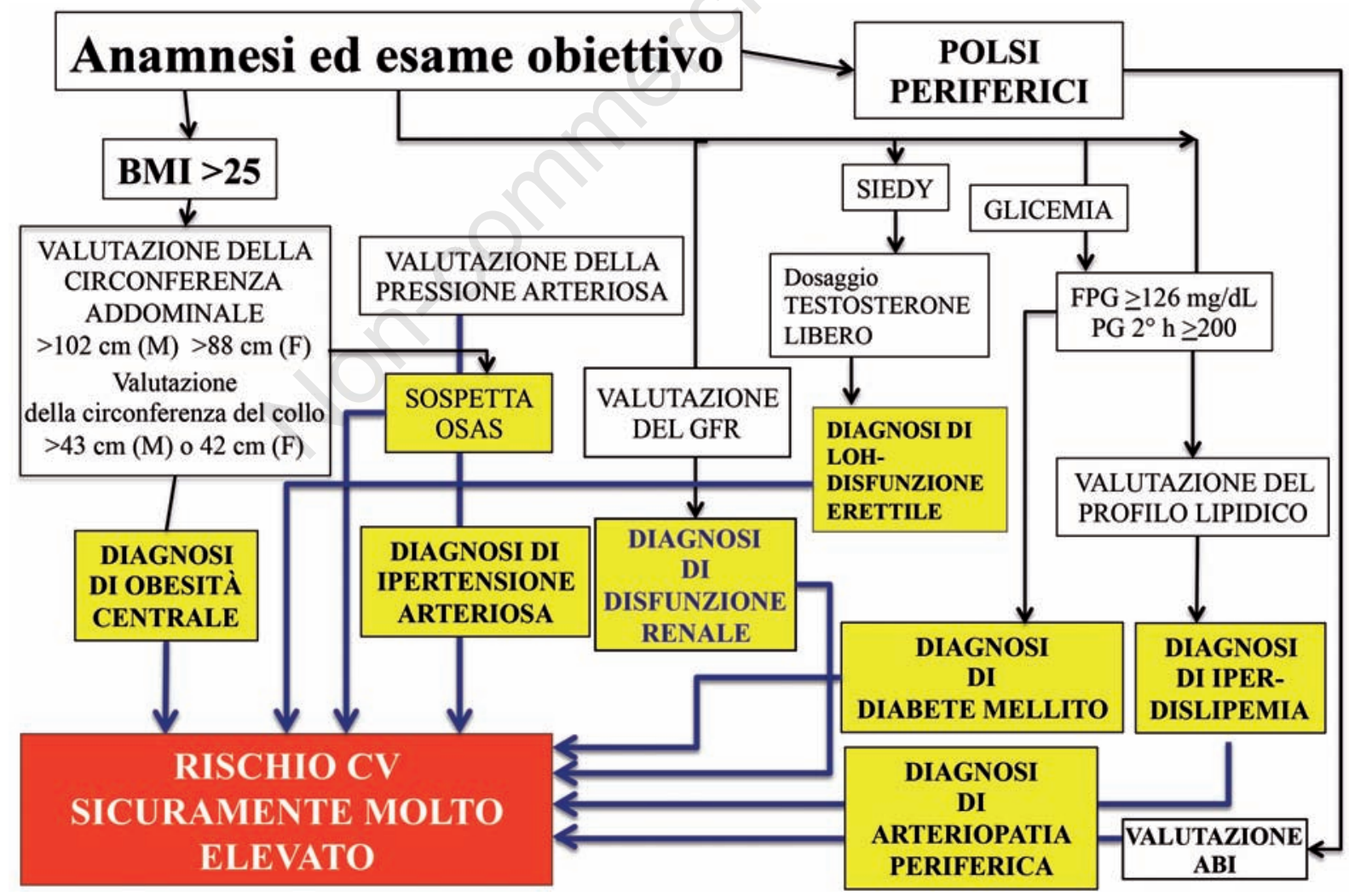

Figura 1. Proposta di un approccio diagnostico frugale, sulla base di un iniziale giudizio clinico multidimensionale, nella valutazione del rischio cardio-vascolare nel paziente internistico complesso. BMI, body mass index; SIEDY, structured interview on erectile dysfunction; OSAS, obstructive sleep apnea syndrome; FPG, fasting plasma glucose; PG, post-prandial blood glucose; LOH, late onset hypogonadism; GFR, glomerular filtration rate; ABI, ankle brachial index. 


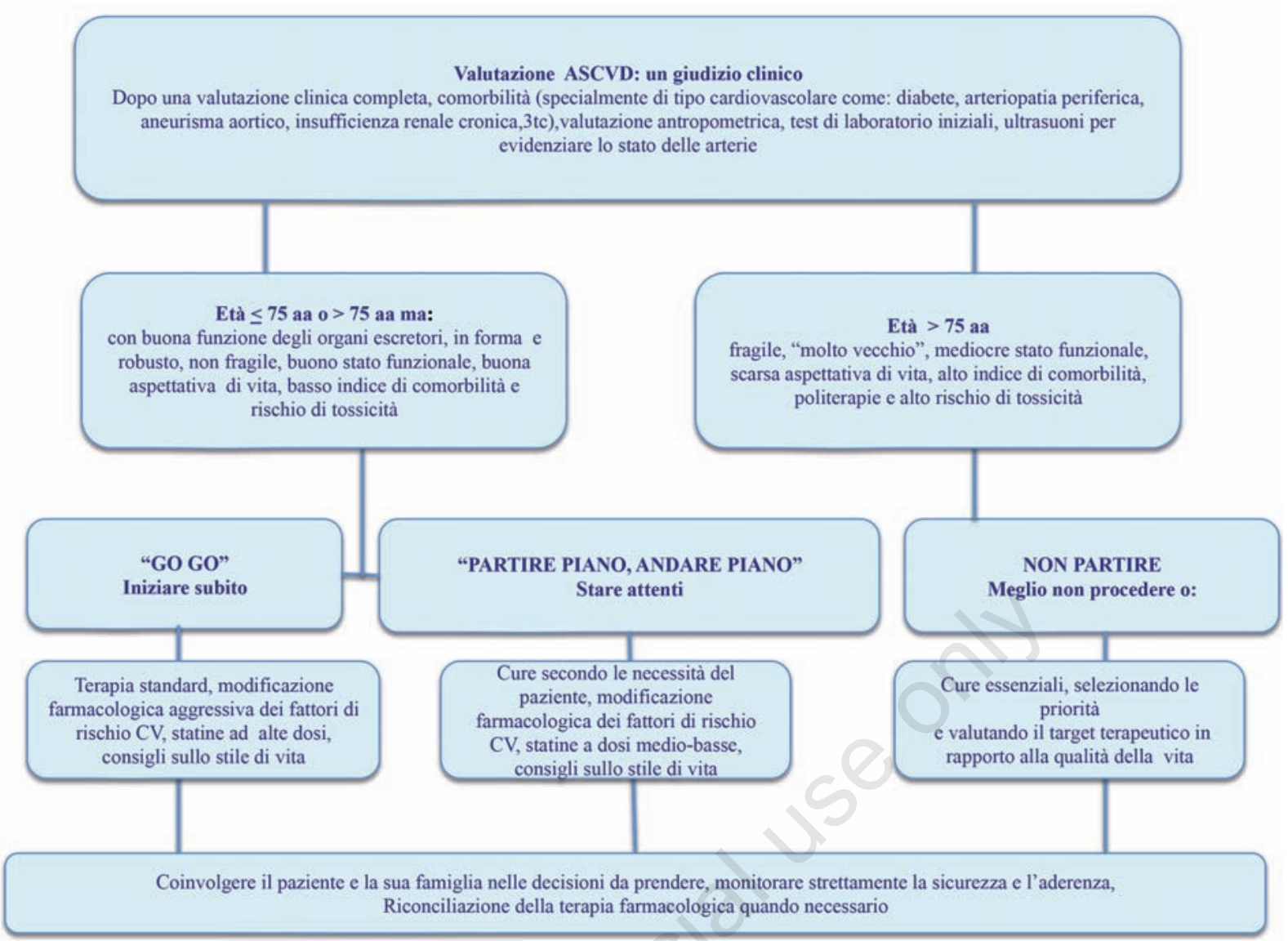

Figura 2. Algoritmo generale per ridurre il rischio di eventi cardiovascolari secondari nella malattia cardiovascolare aterosclerotica. ASCVD, atherosclerotic cardiovascular disease. Tradotta da Campanini et al., $2015 .^{63}$

soggetto, con una valutazione multidimensionale dei problemi in causa, un'attenta supervisione e selezione delle priorità esistenti, nonché sulla reale aderenza a terapie efficaci, con un sistematico esercizio di riconciliazione terapeutica in ogni occasione di cura, implementando il counseling e i trattamenti idonei. ${ }^{82}$ Proprio per evitare derive apodittiche o autoreferenziali, affidiamo alla comunità scientifica il tema della complessità del paziente internistico per un'opportuna discussione, ai fini di un'efficace prevenzione cardiovascolare nel paziente reale complesso.

\section{Bibliografia}

1. Davis MA, Bynum JPW, Sirovich BE. Association between apple consumption and physician visits: appealing the conventional wisdom that an apple a day keeps the doctor away. JAMA Intern Med 2015;175: 777-83.

2. Istituto Superiore di Sanità. Il Progetto Cuore. Disponibile su: http://www.cuore.iss.it/fattori/RischioGlobale Assoluto.asp

3. Lenzi J, Rucci P, Castaldini I, et al. Does age modify the relationship between adherence to secondary prevention medications and mortality after acute myocardial infarction? A nested case-control study. Eur J Clin Pharmacol 2015;71:243-50.

4. Last JM. A Dictionary of epidemiology, 3rd ed. Oxford: Oxford University Press; 1995.

5. Rose GA. The strategy of preventive medicine. Vol. XII. Oxford, New York: Oxford University Press; 1993. pp 138.

6. Manuel DG, Lim J, Tanuseputro P, et al. Revisiting Rose: strategies for reducing coronary heart disease. BMJ 2006;332:659-62.

7. Giustini S, Battaggia A. Statine, rischi e soglie di rischio: domande e risposte aperte ... fra medici di medicina generale. Disponibile su: http://www.farmacovigilanza. org/corsi/070430-01.asp

8. Benjamin EJ, Smith SC Jr, Cooper RS, et al. Task Force \#1 - magnitude of the prevention problem: opportunities and challenges. 33rd Bethesda Conference. J Am Coll Cardiol 2002;40:579-651.

9. Leavell H, Clark E. Preventive medicine for the doctor in his community an epidemiologic approach. 1st ed. New York: McGraw-Hill; 1958.

10. Kuehlein T, Sghedoni D, Visentin G, et al. Quaternary prevention: a task of the general practitioner; 2009. Available from: http://orbi.ulg.ac.be/bitstream/2268/ 177926/1/2010-18-368_ELPS_engl.pdf 
11. Géervas J. Quaternary prevention in the elderly. Rev Esp Geriatr Gerontol 2012;47:266-9.

12. World Health Organization. Prevention of cardiovascular disease - guidelines for assessment and management of cardiovascular risk. Geneva: WHO; 2007. Available from: http://www.who.int/cardiovascu lar_diseases/guidelines/Full\%20text.pdf

13. Mohamad TN, Ali YS. Primary and secondary prevention of coronary artery disease; 2014. Available from: http://emedicine.medscape.com/article/164214overview\#aw2aab6b3

14. Pearson T. New tools for coronary risk assessment what are their advantages and limitations? Circulation 2002;105:886-92.

15. Koenig W. Update on integrated biomarkers for assessment of long-term risk of cardiovascular complications in initially healthy subjects and patients with manifest atherosclerosis. Ann Med 2009;41:332-43.

16. Perk J, De Backer G, Gohlke H, et al. European Guidelines on cardiovascular disease prevention in clinical practice. The Fifth Joint Task Force of the European Society of Cardiology and Other Societies on Cardiovascular Disease Prevention in Clinical Practice (constituted by representatives of nine societies and by invited experts. Eur Heart J 2012;33:1635-701.

17. Comunicato congiunto da parte di ANMCO, FIC, SIC e SISA. Nuove linee guida americane 2013 ACC/AHA Guidelines on the treatment of blood cholesterol to reduce atherosclerotic cardiovascular risk: Confronto con le raccomandazioni EAS/ESC per la gestione delle dislipidemie. Available from: http://www.sisa.it/upload/ Comunicato_Congiunto_lineeguida_EAS_USA.pdf

18. Boyd CM, Leff B, Wolff JL, et al. Informing clinical practice guideline developement and implementation: prevalence of coexisting conditions among adults with coronary heart disease. J Am Geriatr Soc 2011;59:797.

19. Fabbri LM, Luppi F, Beghé B, Rabe KF. Complex chronic comorbidities of COPD. Eur Respir J 2008;31: 204-12.

20. Nozzoli C, Beghè B, Boschetto P, Fabbri LM. Identifying and treating COPD in cardiac patients. Chest 2013;144:723-6.

21. Townsend P, Phillimore P, Beattie A. Health and deprivation: inequality and the north. London: Routledge; 1988.

22. Aryal S, Diaz-Guzman E, Mannino DM. Epidemiology of comorbidities in chronic obstructive pulmonary disease: clusters, phenotypes and outcomes. Ital J Med 2012;6:276-84.

23. Baker JV, Lundgren JD. Cardiovascular implications from untreated human immunodeficiency virus infection. Eur Heart J 2011;32:945-51.

24. Hemkens LG, Bucher HC. HIV infection and cardiovascular disease. Eur Heart J 2014;35:1373-81.

25. Oliveira CP, Kappel CR, Siqueira ER, et al. Effects of hepatitis $\mathrm{C}$ virus on cardiovascular risk in infected patients: a comparative study. Int J Cardiol 2013;164:221-6.

26. Wubiee F, Howell C. Hepatitis C infection is associated with an increase in cardiovascular diseases. Presented at the International Liver Congress, Vienna, Austria. Available from: https://ilc-congress.eu/programmeebook/files/basic-html/page250.html

27. Beltowski J. Leptin and atherosclerosis. Atherosclerosis 2006;189:47-60.
28. Minelli M, Arsieni A, Iadarola G, et al. Rapporto IMID 2007: Documento di base sulla possibile adozione di un modello pluridisciplinare integrato nelle malattie croniche immunomediate. South Immunol J 2007;2:32-43.

29. UK Department of Health - DH Cardiovascular Disease Team. Cardiovascular disease outcomes strategy - improving outcomes for people with or at risk of cardiovascular disease; 2013. Available from: https://www.gov.uk/ government/uploads/system/uploads/attachment_data/file /217118/9387-2900853-CVD-Outcomes_web1.pdf

30. Goff DC Jr, Lloyd-Jones DM, Bennett G, et al. 2013 ACC/AHA guideline on the assessment of cardiovascular risk: a report of the American College of Cardiology/American Heart Association Task Force on Practice Guidelines. Circulation 2014;129:S49-73.

31. Eckel RH, Jakicic JM, Ard JD, et al. 2013 AHA/ACC guideline on lifestyle management to reduce cardiovascular risk: a report of the American College of Cardiology/American Heart Association Task Force on Practice Guidelines. J Am Coll Cardiol 2014;63:2960-84.

32. Stone NJ, Robinson J, Lichtenstein AH, et al. 2013 ACC/AHA guideline on the treatment of blood cholesterol to reduce atherosclerotic cardiovascular risk in adults: a report of the American College of Cardiology/American Heart Association Task Force on Practice Guidelines. Circulation 2014;129:S1-45.

33. Vandvik PO, Lincoff AM, Gore JM, et al. Primary and secondary prevention of cardiovascular disease: antithrombotic therapy and prevention of thrombosis, 9th ed: American College of Chest Physicians EvidenceBased Clinical Practice Guidelines. Chest 2012;141: e637S-68S.

34. Genest J, McPherson R, Frohlich J, et al. 2009 Canadian Cardiovascular Society/Canadian guidelines for the diagnosis and treatment of dyslipidemia and prevention of cardiovascular disease in the adult - 2009 recommendations. Can J Cardiol 2009;25:567-79.

35. ESC/EAS Guidelines for the management of dyslipidaemias: the task force for the management of dyslipidaemias of the European Society of Cardiology (ESC) and the European Atherosclerosis Society (EAS). Atherosclerosis 2011;217S:S1-S44.

36. Virani SS. What is new in the 2013 ACC/AHA guideline on the treatment of blood cholesterol to reduce atherosclerotic risk in adults? Texas Heart Inst J 2014; 41:304-5.

37. Stone NJ, Robinson J, Lichtenstein AH, et al. 2013 ACC/AHA guideline on the treatment of blood cholesterol to reduce atherosclerotic cardiovascular risk in adults: a report of the American College of Cardiology/American Heart Association Task Force on Practice Guidelines. Circulation 2014;129:S1-45.

38. Di Rosa S, Iori I, Vescovo G, et al. Trends in cardiovascular risk factors and their treatment among patients discharged from divisions of internal medicine. The FAPOI-1 and FADOI-2 studies. Ital Heart J 2003;4:460-7.

39. Nardi R, Blasi I, Alduino R, et al. Sindrome metabolica nei pazienti ricoverati in Medicina Interna: risultati dello studio pilota NIMEC (National Internal Medicine Equivalent/Complex C-V-@Risk). Ital J Med 2007;1: 21-31.

40. Expert Panel on Detection, Evaluation, and Treatment 
of High Blood Cholesterol in Adults. Executive summary of the third report of the National Cholesterol Education Program (NCEP) Expert Panel on Detection, Evaluation, and Treatment of High Blood Cholesterol in Adults (Adult Treatment Panel III). JAMA 2001;285: 2486-97.

41. Jensen GL. Inflammation: roles in aging and sarcopenia. JPEN J Parenter Enteral Nutr 2008;32:656-9.

42. Alberti KG, Zimmet P, Shaw J; IDF Epidemiology Task Force Consensus Group. The metabolic syndrome - a new worldwide definition. Lancet 2005;366:1059-62.

43. Gussoni G. Presentazione dei dati preliminari al Consiglio Direttivo FADOI, sulla "Giornata Nazionale della Medicina Interna - Un Metro per la Vita, 7 Giugno 2015", 17 settembre 2015, Bologna.

44. Martin SS, Abd TT, Jones SR, et al. 2013 ACC/AHA cholesterol treatment guidelinewhat was done well and what could be done better. J Am Coll Cardiol 2014;63: 2674-8.

45. Grundy SM, Cleeman JI, Bairey Merz CN, et al. Implications of recent clinical trials for the National Cholesterol Education Program Adult Treatment Panel III guidelines. Circulation 2004;110:227-39.

46. Third National Cholesterol Education Program (NCEP) Expert Panel on Detection, Evaluation, and Treatment of High Blood Cholesterol in Adults (Adult Treatment Panel III). Third report of the National Cholesterol Education Program (NCEP) expert panel on detection, evaluation, and treatment of high blood cholesterol in adults (Adult Treatment Panel III). Final report. Circulation 2002;106:3143-421.

47. Dominguez LJ, Barbagallo M. The cardiometabolic syndrome and sarcopenic obesity in older persons. JCMS 2007;2:183-9.

48. Hales CN, Barker DJ. The thrifty phenotype hypothesis. Br Med Bull 2001;60:5-20.

49. Guardamagna O, Abello F, Cagliero P, Lughetti L. Impact of nutrition since early life on cardiovascular prevention. Ital J Pediatr 2012;38:73.

50. Sipola-Leppnen M, Karvonen R, Tikanmk MI, Matinolli HM. Ambulatory blood pressure and its variability in adults born preterm. Hypertension 2015;65:615-21.

51. Foley RN, Parfrey PS, Sarnak MJ. Clinical epidemiology of cardiovascular disease in chronic renal disease. Am J Kidney Dis 1998;32:S112-9.

52. Corsonello A, Pedone C, Lattanzio F, et al. Does concealed chronic kidney disease predict survival of older patients discharged from acute care hospitals? Rejuvenation Res 2010;13:539-45.

53. Angelico F, del Ben M, Augelletti T, et al. Obstructive sleep apnoea syndrome and the metabolic syndrome in an internal medicine setting. Eur J Intern Med 2010;21: 191-5.

54. Artom N, Pinna G. The erectile dysfunction as a cardiovascular risk factor. Ital J Med 2014;8:210-20.

55. Corrales-Medina VF, Alvarez KN, Weissfeld LA, et al. Association between hospitalization for pneumonia and subsequent risk of cardiovascular disease. JAMA 2015;313:264-74.

56. Garnick MB. Testosterone replacement therapy faces FDA scrutiny. JAMA 2015;316:563-4.

57. US Food and Drug Administration. 2014 Meeting materials of the bone, reproductive, and urologic drugs advisory committee (formerly Advisory Committee for Reproductive Health Drugs (ACRHD)). Available from: http://www.fda.gov/AdvisoryCommittees/Committees MeetingMaterials/Drugs/ReproductiveHealthDrugsAdv isoryCommittee/ucm 404895.htm Accessed: November 20, 2014.

58. Witham MD, Ireland S, Houston JG, et al. Vitamin D therapy to reduce blood pressure and left ventricular hypertrophy in resistant hypertension randomized, controlled trial. Hypertension 2014;63:706-12.

59. Montagnani A, Nardi R, Cercignani M, Verdiani V. Potential role of vitamin $\mathrm{D}$ in prevention of skeletal and extraskeletal diseases in older people. Ital J Med 2015 [In press].

60. Bahekar AA, Singh S, Saha S, et al. The prevalence and incidence of coronary heart disease is significantly increased in periodontitis: a meta-analysis. Am Heart J 2007; 154:830-7.

61. Dietrich T, Sharma P, Walter C, et al. The epidemiological evidence behind the association between periodontitis and incident atherosclerotic cardiovascular disease. J Periodontol 2013;84:S70-84.

62. Nardi R. Prescrizione e valutazione degli esami di laboratorio in ambito cardio-metabolico: strategie integrate per l'identificazione precoce dei pazienti a rischio cardiovascolare. Relazione presso Aula del Rettorato, Università degli Studi di Milano, 6 novembre 2009.

63. Campanini M, Pinna G, Nardi R. The FADOI (Federation of Associations of Hospital Doctors on Internal Medicine) position paper on cardiovascular prevention in the higher risk complex patients. Ital $\mathrm{J}$ Med 2015 [In press].

64. Iacobucci G. NHS plan calls for new models of care and greater emphasis on prevention. BMJ 2014;349:g6430.

65. Giugliano RP. Benefit of adding ezetimibe to statin therapy on cardiovascular outcomes and safety in patients with vs without diabetes: the IMPROVE-IT Trial, at Clinical Trial Update 1 - Cardiovascular diseases: prevention, outcomes, quality. ESC Congress, 30 August 2015, London, UK.

66. The Diabetes Prevention Program Research Group. Impact of intensive lifestyle and metformin therapy on cardiovascular disease risk factors in the diabetes prevention program. Diabet Care 2005;28:888-94.

67. Home P. Cardiovascular disease and oral agent glucoselowering therapies in the management of type 2 diabetes. Diabetes Technol Ther 2012; Suppl 1:S33-42.

68. Mazza A. PUFA Omega-3 e rischio cardiovascolare: dalla teoria alla pratica. Available from: http://www. fadoi.org/allegato_news/1259_Newsletter_FADOI_IBS A_PUFA.pdf

69. Cannon CP, Blazing MA, Giugliano RP, et al. Ezetimibe added to statin therapy after acute coronary syndromes. N Engl J Med 2015;372:2387-97.

70. Giugliano RP. Benefit of adding ezetimibe to statin therapy on cardiovascular outcomes and safety in patients with vs without diabetes: the IMPROVE-IT Trial, at Clinical Trial Update 1 - Cardiovascular diseases: prevention, outcomes, quality. ESC Congress, 30 August 2015, London, UK.

71. Robinson JG, Farnier M, Krempf M, et al. Efficacy and safety of alirocumab in reducing lipids and 
cardiovascular events. N Engl J Med 2015;372:1489-92.

72. Sabatine MS, Giugliano RP, Wiviott SD, et al. Efficacy and safety of evolocumab in reducing lipids and cardiovascular events. N Engl J Med 2015;372:1500-92.

73. Tsimikas S, Viney NJ, Hug SG, et al. Antisense oligonucleotides for treatment of dyslipidemia. Lancet 2015;373:438

74. Tricoci P, Allen JM, Kramer JM, et al. Scientific evidence underlying the ACC/AHA clinical practice guidelines. JAMA 2009;301:831-41.

75. Wise J. Polypharmacy: a necessary evil. BMJ 2013; 347:f7033.

76. Dumbreck S, Flynn A, Nairn M, et al. Drug-disease and drug-drug interactions: systematic examination of recommendations in $12 \mathrm{UK}$ national clinical guidelines. BMJ 2015;350:h949.

77. Benetos A, Labat C, Rossignol P, et al. Treatment with multiple blood pressure medications, achieved blood pressure, and mortality in older nursing home residents.
The PARTAGE Study. JAMA Intern Med 2015;175: 989-95.

78. [No authors listed]. When clinical practice guidelines fail. Lancet 2005;365:1440.

79. Grimshaw J, Eccles M, Thomas R, et al. Toward evidence-based quality improvement, evidence (and its limitations) of the effectiveness of guideline dissemination and implementation, strategies 19661998. J Gen Intern Med 2006;21:S14-20.

80. Campanini M. La Medicina Interna ospedaliera: tra complessità ed intensità assistenziale verso una medicina sostenibile. Quad Ital J Med 2014;2:1-2.

81. Beresford L. The role of hospitalists in preventive medicine. Medscape 2015. Available from: http://www. medscape.com/viewarticle/839159 print

82. Zuccheri P, Manfredi F, Serra A, et al. Medication reconciliation as a tool for reducing polypharmacy at hospital discharge in Internal Medicine Wards. Ital J Med 2014;8:232-7. 


\title{
La patogenesi dell'aterosclerosi
}

\author{
Chiara Nardin, Marcello Rattazzi, Paolo Pauletto \\ Dipartimento di Medicina Interna, Università degli Studi di Padova; Medicina Interna I, Ospedale Cà Foncello, Treviso, Italia
}

\section{Introduzione}

L'aterosclerosi è una patologia cronica multifattoriale che colpisce le arterie di grosso e medio calibro caratterizzata da lesioni intimali focali della tonaca intima che protrudono all'interno del lume arterioso, provocandone un progressivo restringimento. Le lesioni aterosclerotiche presentano generalmente una distribuzione focale all'interno delle singole arterie e interessano contemporaneamente più distretti arteriosi. ${ }^{1}$ Le arterie elastiche e muscolari di grande e medio calibro rappresentano le sedi maggiormente colpite dal processo aterogenico. Le localizzazioni principali in ordine di frequenza sono la porzione distale dell'aorta addominale, le coronarie, le arterie degli arti inferiori (iliache, femorali e poplitee), il tratto discendente dell'aorta toracica, le carotidi, le arterie vertebrali e il circolo del Willis. Mentre le lesioni intimali cominciano a svilupparsi già durante le prime decadi di vita, le manifestazioni cliniche compaiono in età adulta, quando vi è una compromissione significativa del flusso arterioso o in presenza di complicanze. L'infarto miocardico, cerebrale, gli aneurismi aortici e l'arteriopatia periferica rappresentano alcune delle più frequenti conseguenze cliniche della patologia aterosclerotica. ${ }^{2}$

\section{Epidemiologia}

Le patologie cardiovascolari rappresentano la più frequente causa globale di morte $(17,3$ milioni di morti/anno, secondo i dati dell'Organizzazione della Sanità del 2008) equivalente a circa il $30 \%$ della mortalità mondiale. Più dell' $80 \%$ di queste morti per cause

Corrispondente: Paolo Pauletto, Dipartimento di Medicina Interna, Università degli Studi di Padova, Ospedale Cà FoncelloULSS 9, piazza Ospitale 1, 31110 Treviso, Italia.

Tel.: +39.0422.322207 - Fax: +39.0422.22314.

E-mail: paolo.pauletto@unipd.it

Articolo pubblicato secondo la Creative Commons Attribution NonCommercial 3.0 License (CC BY-NC 3.0).

CCopyright C. Nardin et al., 2015

Licensee PAGEPress, Italy

QUADERNI - Italian Journal of Medicine 2015; 3:319-327 cardiovascolari avvengono nei Paesi in via di sviluppo. Sebbene in Europa Occidentale e nel Nord America la mortalità per malattie cardiovascolari negli ultimi anni abbia subito un lento declino dovuto al miglioramento delle strategie terapeutiche e di prevenzione, questa rimane la più frequente causa di morte $\mathrm{e}$ disabilità in tutte le regioni del mondo. ${ }^{3}$

Secondo i dati ISTAT in Italia le patologie cardiovascolari sono responsabili del 35\% dei decessi maschili e del $43 \%$ di quelli femminili. La spesa per gli interventi cardiochirurgici costituisce, da sola, 1' $1 \%$ della spesa sanitaria complessiva. I farmaci che agiscono sul sistema cardiovascolare sono i più utilizzati in assoluto per un importo, da parte del Sistema Sanitario Nazionale, di oltre 5 milioni di euro, il che rappresenta quasi il $93 \%$ della spesa complessiva.

Secondo i dati riportati dall'Istituto Superiore di Sanità nel 2012 l'incidenza di eventi coronarici è maggiore negli uomini rispetto alle donne $(6,3 \%$ per gli uomini e $1,4 \%$ per le donne), come anche l'incidenza di eventi cerebrovascolari $(2,7 \%$ per gli uomini e $1,2 \%$ per le donne). Sia per gli uomini che per le donne i tassi di incidenza aumentano significativamente con l'aumentare dell'età, in particolare al di sopra dei 55 anni. ${ }^{4}$

\section{Fattori di rischio}

I fattori di rischio che predispongono all'aterosclerosi e, conseguentemente alle patologie cardiovascolari, sono stati identificati nel corso di numerosi studi prospettici, condotti in gruppi di popolazione ben definiti, quali ad esempio il multiple risk factor intervention trial. ${ }^{5}$ In particolare si distinguono fattori di rischio modificabili, suscettibili di intervento farmacologico o sullo stile di vita, e fattori di rischio non modificabili (Tabella 1).

$\mathrm{Al}$ di là di alcune condizioni cliniche in cui l'apporto di un singolo fattore di rischio appare predominante, come nel caso dell'ipercolesterolemia familiare omozigote, nella maggioranza dei casi l'insorgenza, la progressione e le complicanze dell'aterosclerosi sono multifattoriali. I fenomeni possono essere spiegati come conseguenza di una complessa interazione fra predisposizione genetica, sia essa relativa all'assetto lipidico, emocoagulativo, metabolico o infiammatorio, e fattori ambientali, come la dieta, lo stile di 
vita e l'esposizione ad agenti infettivi. Lo studio INTERHEART ha comunque dimostrato che più del $90 \%$ degli eventi è attribuibile ai fattori di rischio noti (e modificabili). ${ }^{6}$

\section{Morfologia della placca aterosclerotica}

La classificazione istologica dell'American Heart Association (AHA) suddivide le lesioni aterosclerotiche in sei categorie. ${ }^{7}$

Le strie lipidiche (tipo II secondo la classificazione dell'AHA) rappresentano la lesione aterosclerotica più precoce; sono composte da cellule schiumose infarcite di lipidi e non causano alcuna alterazione di flusso. Esse sono già presenti in tutti $\mathrm{i}$ bambini di età superiore ai 10 anni indipendentemente dal sesso e dalla razza. Gli stessi fattori di rischio associati all'aterosclerosi, sono implicati nella genesi delle strie lipidiche che tuttavia si presentano spesso in aree non suscettibili allo sviluppo di ateromi. Quindi, sebbene le strie lipidiche possano precedere la placca, non tutte sono destinate a tale evoluzione. ${ }^{7}$

Le placche aterosclerotiche avanzate (grado IV, V e VI secondo la classificazione dell'AHA) istologicamente sono costituite da: i) cappuccio fibroso di rivestimento composto da cellule muscolari lisce e da tessuto connettivo relativamente denso; ii) spalle laterali localizzate al di sotto e di lato al cappuccio fibroso costituite da macrofagi, cellule muscolari lisce e linfociti T; iii) core centrale necrotico-lipidico che contiene lipidi (per lo più colesterolo ed esteri di colesterolo), detriti cellulari, depositi di calcio, fibrina, trombi variamente organizzati e proteine plasmatiche.

Soprattutto alla periferia delle lesioni si verificano processi di neovascolarizzazione, spesso ad origine avventiziale. $^{7}$

\section{Patogenesi}

\section{Accumulo di lipidi extracellulare e ossidazione delle lipoproteine a bassa densità}

Studi sperimentali hanno dimostrato che, in seguito all'aumento della concentrazione plasmatica di colesterolo, le lipoproteine a bassa densità (LDL) infiltrano la tonaca intima e interagiscono con i proteoglicani della matrice extracellulare. ${ }^{3}$ Il legame delle lipoproteine ai proteoglicani comporta l'accumulo delle stesse nella tonaca intima e aumenta la loro suscettibilità all'ossidazione. L'ossidazione delle LDL (ox-LDL), prima nella porzione lipidica e successivamente in quella proteica, è favorita dalle specie reattive dell'ossigeno (reactive oxygen species [ROS]) che si generano durante il passaggio nella tonaca intima. Anche le NADH/NADPH ossidasi prodotte dalle cellule endoteliali e le lipossigenasi espresse dai leuco- citi, contribuiscono a tale processo. ${ }^{8}$ L'accumulo delle LDL nello spazio subendoteliale è favorito quando esse sono piccole e dense. Si tratta dell'alterazione qualitativa delle LDL più frequentemente riscontrata nei pazienti con sindrome metabolica o diabete. Il nostro gruppo ha dimostrato come, a parità di livelli di LDL in circolo, le lesioni aterosclerotiche di pazienti con LDL piccole e dense presentano uno sviluppo caratterizzato da un maggior profilo di fenomeni infiammatori e di instabilità della placca..$^{9,10}$

\section{Attivazione endoteliale}

Un endotelio integro generalmente è impermeabile al passaggio dei leucociti, produce vasodilatatori endogeni, favorisce la fibrinolisi ed esprime enzimi, quali la superossido dismutasi, che degradano le ROS. Il danno endoteliale cronico o ripetuto indotto da alterazioni emodinamiche (il flusso laminare induce l'espressione di geni ateroprotettivi) e dall'esposizione a fattori proaterogenici quali le ox-LDL o proinfiammatori quali le citochine, comporta l'espressione endoteliale di molecole di adesione leucocitaria. ${ }^{11}$ Per esempio, la molecola di adesione vascolare di tipo 1 (vascular cell adhesion molecule-1) rappresenta un marker di attivazione endoteliale. Essa interagisce specificamente con molecole quali la molecola di adesione intercellulare di tipo 1 (intercellular adhesion molecule-1) e l'integrina $\alpha 4 \beta 1$ (very antigen-4) espresse dalle classi leucocitarie (monociti e linfociti T) riscontrabili precocemente negli ateromi. Anche la P-selectina è una molecola di attivazione endoteliale implicata nell'aterogenesi. ${ }^{11}$

\section{Reclutamento di leucociti all'interno della tonaca intima e differenziazione in macrofagi}

L'espressione endoteliale di molecole di adesione leucocitaria e la produzione di chemochine quali la proteina monocyte chemoattractant protein-1, determina il passaggio dei monociti nella tonaca intima e

Tabella 1. Fattori di rischio per lo sviluppo dell'aterosclerosi.

\begin{tabular}{ll}
\hline Non modificabili & Modificabili \\
\hline Familiarità & Dislipidemie \\
\hline Sesso maschile & Ipertensione arteriosa \\
\hline Menopausa & Diabete mellito \\
\hline Età & Fumo di sigaretta \\
\hline & Obesità \\
\hline & Sedentarietà \\
\hline Iperomocisteinemia \\
\hline
\end{tabular}


il loro accumulo nello spazio subendoteliale. ${ }^{11}$ Qui vanno incontro a proliferazione e a differenziazione in macrofagi. Diversi mediatori regolano tale processo, il più importante dei quali è il fattore stimolante le colonie monocitarie (monocyte-colony stimulating factor). L'accumulo dei fagociti mononucleati nella lesione primitiva non è mediato solo dall'espressione di molecole di adesione e dalle chemochine, ma anche da segnali di mantenimento all'interno della placca. ${ }^{3}$ Alcuni studi infatti hanno dimostrato che la netrina-1, prodotta dai macrofagi subendoteliali, agisce mediante segnali paracrini e autocrini mantenendo le cellule infiammatorie all'interno della lesione aterosclerotica. ${ }^{12}$

\section{Accumulo di lipidi intracellulare e formazione delle cellule schiumose}

La differenziazione dei monociti in macrofagi si associa all'aumentata espressione dei recettori scavenger (SRs). Questa classe di pattern recognition receptors (PRRs) comprende le proteine di transmembrana scavenger receptor A (SR-A), thrombospondin receptor, e lectin-like oxidized low-density lipoprotein receptor-1, che mediano la fagocitosi di endotossine, corpi apoptotici, frammenti microbici e ox-LDL. Il processo di endocitosi delle ox-LDL mediato dai recettori scavenger non è saturabile, perciò i macrofagi accumulano lipidi intracellulari e diventano cellule schiumose (foam cells). Il rigurgito di radicali liberi (regurgitation during feeding) generato durante la fagocitosi, favorisce l'ulteriore ossidazione delle LDL, mediante feedback positivo. L'accumulo progressivo di macrofagi e cellule schiumose esita nella progressione della lesione. Fenomeni di morte cellulare contribuiscono alla formazione di un core necrotico, costituito prevalentemente da corpi apoptotici e detriti cellulari. $^{3}$

\section{Migrazione e proliferazione delle cellule muscolari lisce}

L'attivazione endoteliale e il reclutamento dei leucociti intervengono nelle fasi iniziali dello sviluppo dell'ateroma. Le cellule muscolari lisce (SMC) della tonaca media sono coinvolte nella successiva evoluzione in placca aterosclerotica matura. Queste cellule, normalmente quiescenti, si attivano e migrano nella tonaca intima. Qui vanno incontro a proliferazione e acquisizione di un fenotipo di tipo sintetico, deputato alla sintesi di componenti della matrice extracellulare. ${ }^{13,14}$ Molti fattori di crescita sono implicati nella proliferazione e migrazione delle SMC. Il platelet-derived growth factor (PDGF), prodotto dalle cellule endoteliali, macrofagi e piastrine che hanno aderito all'endotelio attivato, è il principale fattore coinvolto. L'accumulo subendoteliale delle SMC e la loro proliferazione durante l'aterogenesi e la crescita della placca, non avviene in modo lineare, ma è variabile nel tempo. Anche la sintesi di matrice extracellulare non presenta andamento lineare ma è regolata dalla presenza di enzimi catalitici quali le metalloproteinasi (MMPs). La dissoluzione della matrice infatti stimola l'ulteriore migrazione delle SMC nella tonaca intima mediante feedback positivo. La progressione di questi processi determina lo sviluppo della placca fibro-adiposa matura avanzata che spesso va incontro a neovascolarizzazione periferica e calcificazione. Molti fattori angiogenetici infatti sono espressi nella placca. Tra i principali si annoverano acidic and basic fibroblast growth factors, vascular endothelial growth factor e oncostatina $\mathrm{M}^{8}{ }^{8}$

Le SMC, che contribuiscono alla formazione del cappuccio fibroso della placca matura, ne determinano anche la stabilità; maggiore infatti è lo spessore del cappuccio fibroso, maggiore è la stabilità della placca. È stato dimostrato anche dal nostro gruppo come le placche carotidee instabili presentino un ridotto contenuto di SMC rispetto a quelle stabili. ${ }^{13,14} \mathrm{La}$ quantità di tali cellule viene regolata anche dai processi di differenziazione dei miofibroblasti avventiziali che possono migrare dalla tonaca avventizia all'intima. ${ }^{15}$

\section{Ruolo dell'apoptosi}

L'accumulo di componenti cellulari, lipidi e matrice extracellulare contribuisce allo sviluppo della placca fibroadiposa che può crescere fino al progressivo restringimento del lume arterioso. L'incremento dei fenomeni apoptotici descritti, controbilanciando la proliferazione cellulare, potrebbe portare alla regressione della placca. Al contrario, un loro decremento favorirebbe lo sviluppo dell'iperplasia intimale. Differenti studi hanno dimostrato la presenza di un meccanismo antiapoptotico coinvolto nell'accumulo delle cellule schiumose. L'espressione del recettore SR-A, mediante alterate vie di trasduzione del segnale, determina una resistenza da parte delle cellule schiumose all'apoptosi indotta dalle ox-LDL. Nella placca aterosclerotica avanzata vi è tuttavia un'elevata percentuale di corpi apoptotici, che non si associano a regressione della lesione. Tale fenomeno si sviluppa perché i meccanismi di rimozione dei corpi apoptotici all'interno della placca sono carenti. L'accumulo di lipidi intracellulari infatti riduce la capacità dei macrofagi di rimuovere le cellule apoptotiche, che possono stabilizzarsi nella placca anche mediante interazioni tra proteine, acidi nucleici e carboidrati. ${ }^{16}$

\section{Il ruolo dell'infiammazione}

La malattia aterosclerotica rappresenta a tutti gli effetti una patologia infiammatoria cronica. ${ }^{17}$ Studi sperimentali infatti hanno dimostrato che l'infiammazione regola la genesi, la progressione e le complicanze cli- 
niche della placca ateromatosa. Il ruolo delle citochine, messaggeri infiammatori, fornisce una spiegazione di come i fattori di rischio possano alterare la biologia delle arterie e generare un background sistemico che favorisce il processo aterogenico. La scoperta delle basi immunologiche della cosiddetta allograft arteriosclerosis ha inoltre dimostrato che l'infiammazione da sola può condurre all'iperplasia arteriosa, anche in assenza dei tradizionali fattori di rischio. ${ }^{18}$

Durante l'aterogenesi si verifica una continua interazione tra immunità innata, immunità acquisita $\mathrm{e}$ cellule endoteliali. I linfociti T rispondono alla stimolazione antigenica elaborando citochine quali interferone $\gamma$ e tumor necrosis factor $\alpha$ che attivano le cellule effettrici dell'immunità innata. Queste a loro volta rilasciano mediatori dell'infiammazione quali interleuchina (IL)-1, IL-6, PDGF e transforming growth factor $\alpha(\mathrm{TGF} \alpha)$. Anche l'endotelio, in risposta alle ox-LDL e all'angiotensina II, anche al di fuori dell' ipertensione, elabora molecole pro-infiammatorie. Si ha dunque la produzione di segnali infiammatori autocrini e paracrini che accompagnano l'avvio del processo aterogenico. ${ }^{18,19}$

\section{Immunità innata}

Oltre ai monociti/macrofagi e i PRRs, di cui sopra si è discusso, anche altre cellule intervengono nel processo aterogenico e nelle sue complicanze. I mastociti e i polimorfonucleati si accumulano prevalentemente a livello del sito di rottura della placca, nella cui genesi essi sembrano avere un ruolo attivo. Frammenti di complemento sono stati inoltre riscontrati a livello della lesione precoce, ma il loro ruolo è contradditorio. Recenti studi suggeriscono una funzione protettiva del complemento attraverso le vie di attivazione classica e mediata da lectina. ${ }^{3,20}$

Anche le cellule dendritiche, normalmente presenti nelle tonache intima e avventizia dei vasi arteriosi, risultano incrementate nella placca aterosclerotica. Queste possono sia internalizzare le ox-LDL e diventare cellule schiumose, sia attivare i linfociti $\mathrm{T}$ mediante presentazione dell'antigene. $^{3}$

\section{Immunità acquisita}

Come già detto, i linfociti $\mathrm{T}$ hanno un ruolo decisivo nella regolazione dell'infiammazione durante l'aterogenesi. In particolare i linfociti Th1 CD4+ accelerano tale processo, mentre i linfociti T-regolatori, mediante la secrezione di transforming growth factor- $\beta$ (TGF $\beta)$ e di IL-10, lo limitano. Anche i linfociti B modulano gli eventi infiammatori. Evidenze sperimentali suggeriscono che le cellule B1, deputate alla produzione di anticorpi naturali, abbiano un ruolo protettivo, mentre i linfociti B2, accelerino l'aterogenesi. ${ }^{16}$ Le LDL e la heat shock protein-60 (Hsp60), sembrano essere gli autoantigeni maggiormente coin- volti nella stimolazione antigenica che attiva l'immunità acquisita. Le cellule endoteliali esposte a stress metabolico o ossidativo esprimono Hsp60 e studi sperimentali condotti sui topi hanno dimostrato che l'immunizzazione contro Hsp60 accelera l'aterosclerosi. ${ }^{3}$

\section{Inflammosoma}

L'accumulo di colesterolo intracellulare conduce alla formazione di cristalli, il cui sviluppo dipende dal rapporto tra colesterolo libero ed esterificato e dall'efflusso del colesterolo verso recettori extracellulari (come l'apoliproproteina E). I microcristalli di colesterolo possono condurre all'attivazione dell'inflammosoma (HLRP3), all'interno dei macrofagi. L'attivazione di questo complesso multiproteico è un importante amplificatore della risposta infiammatoria cellulare e rappresenta quindi un possibile pathway patogenetico che correla l'ipercolesterolemia con il processo infiammatorio cronico che caratterizza l'aterosclerosi. È stato infatti dimostrato che l'inattivazione di componenti dell'inflammosoma NLRP3 si accompagna ad una riduzione dell'aterosclerosi in modelli murini. ${ }^{3}$

\section{Ruolo della proteina C reattiva}

La proteina $\mathrm{C}$ reattiva $(\mathrm{CRP})$ è una pentraxina a catena corta ed è considerata la proteina di fase acuta più importante. Prodotta dal fegato, aumenta rapidamente nel plasma in risposta agli stimoli infiammatori, mediati principalmente dall'IL-6. Il test denominato high-sensivity CRP (hs-CRP) è in grado di individuare livelli più ridotti ed è stato utilizzato come marcatore di rischio cardiovascolare indipendente. Aumentati livelli di hs-CRP sono predittivi di eventi cardiovascolari sia primari che secondari in aggiunta ai biomarkers di rischio tradizionali. ${ }^{3}$ Studi in vitro hanno inoltre dimostrato che la CRP può essere coinvolta direttamente nell'attivazione di meccanismi infiammatori a livello della placca (quali la produzione di molecole di adesione endoteliale, l'attivazione dei monociti, e delle SMC).$^{19}$ Tuttavia il ruolo della CRP come fattore patogenetico nell'uomo rimane incerto. Un potere predittivo quale marker di infarto miocardico è stato riconosciuto anche ad altre pentraxine a catena lunga. ${ }^{21}$

\section{Calcificazione}

La deposizione di calcio nelle lesioni aterosclerotiche avanzate rappresenta un evento frequente. La relazione esistente fra la calcificazione intimale e l'instabilità della placca è tuttora dibattuta. Alcuni studi istopatologici, infatti, mostrano che le lesioni aterosclerotiche calcifiche sono più instabili e vanno maggiormente incontro a rottura con aumentato rischio di complicanze trombotiche. ${ }^{22}$ 
Al contrario altri studi, tra cui alcuni condotti utilizzando l'intravascular ultrasound system (IVUS), correlano le lesioni instabili ad una minore deposizione di calcio suggerendone un ruolo protettivo. ${ }^{23-25}$ Alcuni Autori interpretano questi dati discordanti ritenendo che il ruolo della calcificazione intimale sia di tipo bifasico, determinando instabilità della placca solo nelle fasi iniziali, quando l'interfaccia tra aree calcifiche e non calcifiche, è maggiore.

Alcuni studi descrivono meccanismi fisiopatologici comuni tra la calcificazione della placca e il rimodellamento osseo ${ }^{26}$ e differenti ipotesi patogenetiche sono state formulate per spiegare l'origine della calcificazione intimale.

Secondo la teoria dell'inibizione la perdita di alcune molecole espresse a livello delle pareti vascolari induce la deposizione di calcio. Tra queste si annoverano soprattutto la matrix-gla-proteins, l'psteopontina e il pirofosfato. ${ }^{27}$

Secondo la teoria dei complessi cristallini circolanti alcuni dei fattori coinvolti nell'inibizione della calcificazione vascolare (in particolare la fetuina A) fanno parte di complessi circolanti di nucleazione, rilasciati durante il turnover osseo, i cui livelli sierici correlano con la calcificazione della parete vascolare.

La teoria dell'osteogenesi si basa sul fatto che sono stati descritti dei precursori condro-osteogenici all'interno della placca sin dalle fasi precoci del processo di calcificazione. Non è chiaro se questi elementi cellulari derivino dalla transdifferenziazione delle SMC, oppure dall'attivazione/reclutamento di cellule progenitrici con potenziale osteogenico (circolanti o residenti nella parete vascolare). ${ }^{28-31}$

\section{Complicanze}

La genesi e lo sviluppo della placca aterosclerotica avvengono generalmente nell'arco di anni o decenni, durante i quali l'individuo è asintomatico. In questa fase la crescita della lesione avviene in modo discontinuo con periodi di relativa quiescenza, ed altri di rapido incremento con progressiva riduzione del lume arterioso e stenosi. ${ }^{8}$ La placca fibroadiposa matura può infine evolvere in una lesione complicata che diviene clinicamente sintomatica.

\section{Trombosi luminale}

I pazienti con sindrome coronarica acuta si presentano con angina instabile, infarto miocardico acuto con o senza elevazione del tratto ST, o morte cardiaca improvvisa. La maggior parte di queste sindromi è il risultato dello sviluppo di una trombosi luminale su placca aterosclerotica. Studi condotti post mortem hanno descritto tre differenti entità morfologiche determinanti lo sviluppo di trombosi luminale: la rottura
(55-65\% dei casi), l'erosione (30-35\% dei casi) e i noduli calcifici (2-7\% dei casi). ${ }^{32}$

\section{Rottura di placea}

La rottura della placca determina l'esposizione dei lipidi e del fattore tissutale del core necrotico sottostante; la conseguente attivazione della cascata coagulativa genera il trombo luminale. Il fibroateroma a cappuccio sottile (thin cap fibroatheroma [TCFA]), definito anche placca instabile o vulnerabile, rappresenta la lesione che maggiormente va incontro a rottura. Essa si caratterizza per la presenza di un core necrotico centrale sovrastato da un sottile cappuccio fibroso (differenti studi hanno dimostrato che il 95\% delle placche rotte presenta un cappuccio fibroso di spessore $<65 \mu \mathrm{m})^{33}$ infiltrato da macrofagi e linfociti $\mathrm{T}$ (Figura 1). ${ }^{34} \mathrm{Il}$ cappuccio fibroso è costituito per lo più da fibre collagene di tipo $\mathrm{I} e$ da poche SMC. Si ritiene che la rottura della placca avvenga generalmente nel punto di maggior debolezza, ossia a livello delle spalle laterali. Differenti fattori promuovono la progressiva degradazione del cappuccio, tra i quali la produzione di MMPs (MMP-1, MMP-8 e MMP-13), l'elevato shear stress, l'elevato rapporto tra colesterolo libero ed esterificato, l'accumulo di ferro e la morte cellulare dei macrofagi. ${ }^{32}$

Come per la genesi e la progressione della placca, l'infiammazione regola anche l'integrità del cappuccio fibroso. Le citochine pro-infiammatorie infatti, da un lato incrementano la produzione di MMPs, dall'altro stimolano l'espressione di fattori tissutali procoagulanti (per es., CD40 ligando). ${ }^{18}$

Anche le emorragie intralesionali promuovono la fissurazione della placca. Il rivestimento endoteliale discontinuo e la mancanza di muscolatura liscia dei microvasi ne facilitano la rottura. Le emorragie intralesionali rappresentano uno stimolo infiammatorio, ampliando il core necrotico e aumentando la densità dei macrofagi. Infatti l'emoglobina libera può legare e inattivare l'ossido nitrico, che normalmente riduce l'espressione di molecole di attivazione endoteliale. ${ }^{32}$

\section{Erosione di placea}

L'erosione della placca comporta la formazione di un trombo che si sviluppa direttamente a contatto con una tonaca intima ricca di SMC e proteoglicani della matrice, ma priva del rivestimento endoteliale. L'ispessimento medio intimale (patological intimal thickening) e il TCFA, si riscontrano con uguale frequenza nella placca erosa. Mentre nella rottura di placca la tonaca media risulta spesso degradata, soprattutto a livello della membrana elastica interna, nell'erosione questa rimane intatta. L'assenza di macrofagi e linfociti a livello del sito di erosione dimostra che l'infiammazione svolge in questo caso un ruolo marginale. ${ }^{32}$

In uno studio condotto su pazienti vittime di morte 
cardiaca improvvisa, Virmani et al., hanno evidenziato che più dell' $85 \%$ dei trombi coronarici derivati da erosione della placca mostravano segni tardivi di risoluzione del trombo, rispetto a quelli originati da rottura della placca, presenti in meno del $50 \%{ }^{35}$

\section{Noduli calcifici}

I noduli calcifici si sviluppano solitamente nelle arterie calcifiche in soggetti generalmente maschi e anziani. Sono circondati da fibrina e contengono un piccolo trombo piastrinico luminale non occludente. Sono generalmente eccentrici, protrudono nel lume, in assenza di endotelio e collagene. Sebbene il processo rimanga sconosciuto, la presenza di fibrina, rari osteoclasti e cellule infiammatorie, indica che la frammentazione dei noduli calcifici, con conseguente emorragia intralesionale, possa attivare la trombosi intralesionale. ${ }^{32}$

\section{Ristenosi}

Nell'ambito del trattamento della patologia aterosclerotica, la ristenosi rappresenta tuttora una significativa limitazione all'efficacia delle procedure di rivascolarizzazione percutanea. L'incidenza di tale complicanza è variabile e dipende dal calibro del vaso coinvolto e dalla procedura stessa di rivascolarizzazione. Mentre per lo sviluppo della lesione aterosclerotica primitiva $\mathrm{i}$ fattori di rischio tradizionali svolgono un ruolo determinante, nella ristenosi e nello sviluppo della neointima essi sembrano avere una minore rilevanza. Il rimodellamento delle tonache avventiziale, media e intima sembra infatti rappresentare una risposta alle procedure di rivascolarizzazione. ${ }^{34,36,37}$ Tra i fattori potenzialmente coinvolti nel processo di ristenosi si annoverano l'infezione, l'infiammazione, l'espressione di fattori di crescita e citochine, e la produzione di MMPs, ovvero di fattori che agiscono localmente a livello del vaso coinvolto. ${ }^{36} \mathrm{Il}$ follow up in vivo, effettuato mediante tecniche ultrasonografiche ad elevata risoluzione combinate con lo studio morfologico delle placche sottoposte a endarterectomia carotidea, ha permesso di correlare le caratteristiche della placca con il rischio di ristenosi. In particolare, le evidenze sperimentali dimostrano che i macrofagi e i lipidi svolgono un ruolo determinante nella patogenesi dell'ateroma, ma non nello sviluppo della neointima dopo endarterectomia carotidea. ${ }^{34,37}$

Viceversa gli studi condotti su campioni di endar-

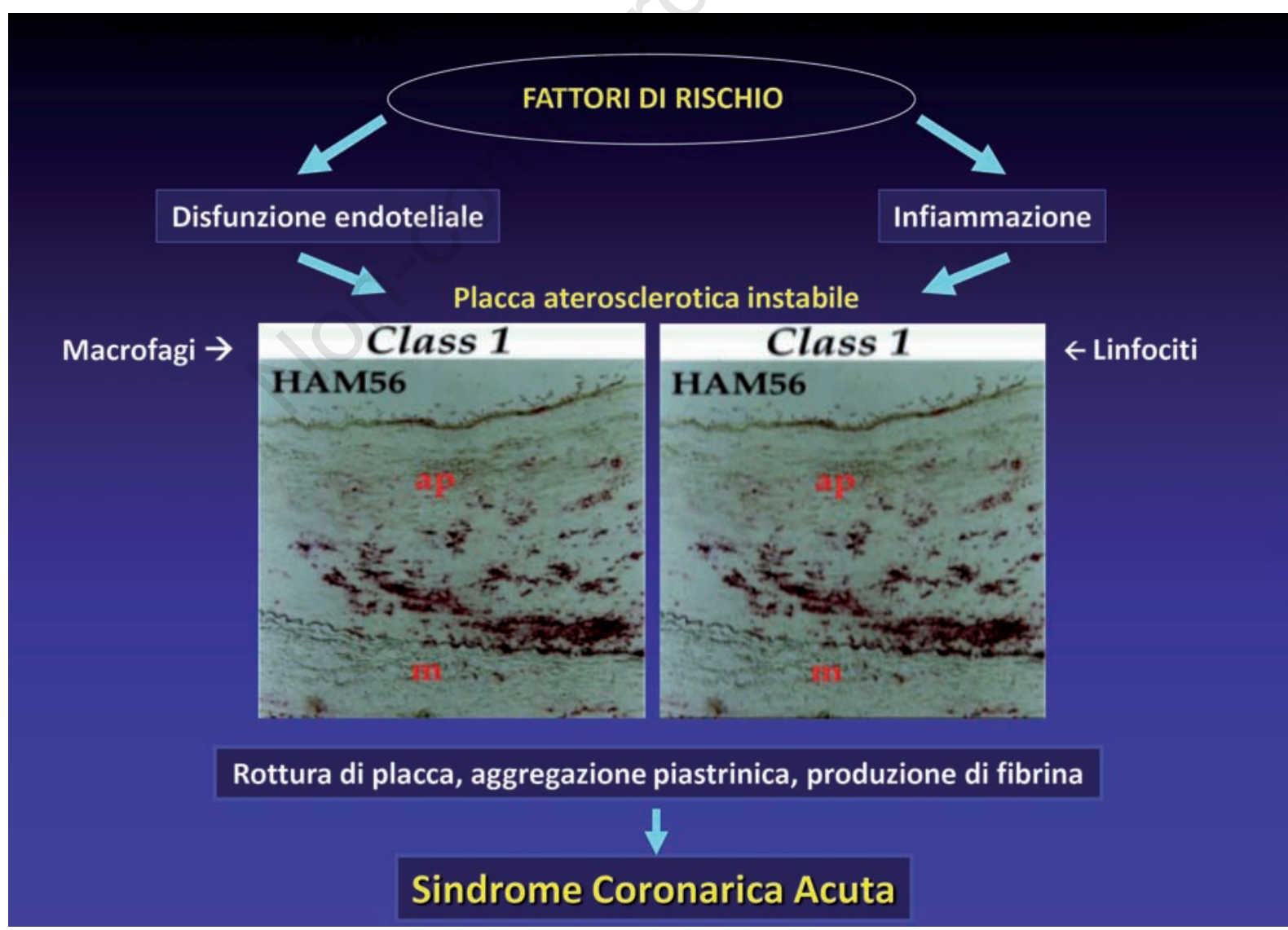

Figura 1. Patogenesi ed evoluzione della placca instabile. Modificata da Pauletto et al., 2000.34 
terectomia coronarica avvalorano la teoria che lesioni primitive ricche di lipidi e cellule infiammatorie siano associate ad un aumentato rischio di ristenosi nel distretto coronarico. ${ }^{36}$ Ciò indica come in distretti arteriosi diversi lo sviluppo di ristenosi dipenda da meccanismi legati a tipi cellulari differenti.

In questo ambito uno studio condotto dal nostro gruppo ha permesso di osservare come le placche carotidee primitive di pazienti che durante il follow-up sviluppavano ristenosi (neointima $>1.3 \mathrm{~mm}$ ), avevano un maggior contenuto di SMC a fronte di un ridotto numero di macrofagi e linfociti. Inoltre lo strato medio sottostante la placca si caratterizzava per una riduzione delle SMC con aumento della percentuale di quelle con fenotipo indifferenziato. Quest'ultima peculiare caratteristica suggerisce che queste cellule sono in grado di migrare e accumularsi nella tonaca intima in seguito al processo di rivascolarizzazione. ${ }^{15}$

Due fenotipi cellulari sono infatti coinvolti nello sviluppo della neointima, ovvero i miofibroblasti e le SMC indifferenziate. Queste ultime possono originare, oltre che dai miofibroblasti stessi, anche da un processo di dedifferenziazione delle SMC. La migrazione nello spazio subendoteliale di miofibroblasti e SMC indifferenziate in risposta al danno vascolare conduce allo sviluppo della neointima, responsabile del processo di ristenosi. ${ }^{15}$

Da queste evidenze sperimentali, basate sullo studio di campioni di endarterectomia nell'uomo, si evince dunque che la composizione cellulare della placca aterosclerotica primaria rappresenta un fattore di rischio indipendente per lo sviluppo di ristenosi.

\section{Statine e stabilizzazione della placca aterosclerotica}

Nell'ambito delle strategie di prevenzione primaria e secondaria degli eventi cardiovascolari, le statine rivestono oggi un ruolo ben consolidato, determinando una significativa riduzione della mortalità e morbilità ad essi correlate. Gli effetti fisiopatologici che questi farmaci esercitano sulla risposta infiammatoria e sulla morfologia della placca aterosclerotica sono tuttavia oggetto di dibattito. Non è chiaro infatti se le alterazioni morfologiche indotte dalle statine sulla placca siano dovute principalmente alla riduzione dei livelli di LDL, ai loro effetti pleiotropici antinfiammatori, oppure ad entrambi questi fattori. Molti studi sperimentali infatti dimostrano che, oltre al noto effetto di riduzione delle LDL, le statine presentano delle proprietà antinfiammatorie e antitrombotiche che sono indipendenti dall'effetto ipolipemizzante..$^{38}$

Alcuni studi prospettici hanno esaminato l'impatto delle statine sulla stabilità della placca carotidea. I pazienti sottoposti a trattamento intensivo con statine presentavano una placca più stabile, con un ridotto contenuto di cellule infiammatorie quali macrofagi e linfociti T, e un maggior numero di SMC..$^{39-41}$ Uno studio condotto dal nostro gruppo ha confrontato pazienti in trattamento ipocolesterolemizzante non statinico con pazienti in terapia con statine. Dopo tre mesi di terapia, in coloro che assumevano statine, si evidenziava una placca più stabile con un ridotto contenuto di macro-

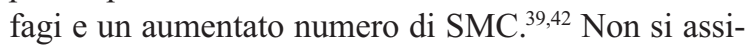
steva invece ad alcuna modifica dei linfociti T. Altri studi hanno evidenziato una significativa riduzione degli infarti miocardici peri-procedurali nei pazienti trattati con statine nei trenta giorni successivi a procedure cardiache invasive. Molte evidenze cliniche supportano quindi il ruolo antiinfiammatorio e di stabilizzazione delle statine sulla placca aterosclerotica, già dopo pochi mesi di terapia. Tale effetto è dimostrato dalla composizione istologica della placca che va incontro ad una significativa riduzione dei tipi cellulari e delle citochine responsabili delle complicanze..$^{38}$ Un'ulteriore riprova dell'impatto positivo delle statine sulla placca deriva dagli studi condotti da Nissen et al. usando l'IVUS. L'uso di questa tecnologia ha permesso di dimostrare in vivo, nell'uomo e individualmente, che il trattamento con statine è in grado di ridurre la dimensione della lesione aterosclerotica. Tuttavia questi studi non sono in grado di fornire dati diretti sulla stabilità della lesione stessa. ${ }^{43}$

In conclusione, la composizione cellulare della placca è modulata significativamente dal livello di LDL e dalla loro riduzione, mentre le proprietà antinfiammatorie delle statine e gli effetti di stabilizzazione della placca indipendenti dalle LDL sono ad oggi solo ipotizzabili sulla base di alcuni studi. Pertanto, il raggiungimento di livelli di LDL progressivamente più bassi resta attualmente il target terapeutico più efficace per la stabilizzazione delle lesioni aterosclerotiche e la conseguente riduzione del rischio cardiovascolare. ${ }^{38}$

\section{Bibliografia}

1. Puato MFE, Pauletto P, Pessina AC. La malattia aterosclerotica. In: Crepaldi G, Baritussio A, eds. Malattie del cuore e dei vasi. Padova: Editore Piccin; 2002.

2. Stary HC, Chandler AB, Glagov S, et al. A definition of initial, fatty streak, and intermediate lesions of atherosclerosis. A report from the Committee on Vascular Lesions of the Council on Arteriosclerosis, American Heart Association. Arterioscler Thromb 1994;14:840-56.

3. Libby P, Lichtman AH, Hansson GK. Immune effector mechanisms implicated in atherosclerosis: from mice to humans. Immunity 2013;38:1092-104.

4. Appelgren EC, Luzi P, eds. Convegno La salute degli italiani nei dati del Centro Nazionale di Epidemiologia, Sorveglianza e Promozione della Salute (CNESPS). Istituto Superiore di Sanità Roma, 16-17 giugno 2011. Atti. Roma: Istituto Superiore di Sanità; 2012. (Rapporti ISTISAN 12/05). p 10.

5. Stamler J, Neaton JD, Cohen JD, et al. Multiple risk fac- 
tor intervention trial revisited: a new perspective based on nonfatal and fatal composite endpoints, coronary and cardiovascular, during the trial. J Am Heart Assoc 2012; 1:e003640.

6. Yusuf S, Hawken S, Ounpuu S, et al. Effect of potentially modifiable risk factors associated with myocardial infarction in 52 countries (the INTERHEART study): case-control study. Lancet 2004;364:937-52.

7. Stary HC, Chandler AB, Dinsmore RE, et al. A definition of advanced types of atherosclerotic lesions and a histological classification of atherosclerosis. A report from the Committee on Vascular Lesions of the Council on Arteriosclerosis, American Heart Association. Arterioscler Thromb Vasc Biol 1995;15:1512-31.

8. Zipes DP, Libby P, Bonow RO, Braunwald E. Heart disease. Philadelphia, PA: Elsevier; 2005.

9. Faggin E, Zambon A, Puato M, et al. Association between the $-514 \mathrm{C} \rightarrow \mathrm{T}$ polymorphism of the hepatic lipase gene promoter and unstable carotid plaque in patients with severe carotid artery stenosis. J Am Coll Cardiol 2002;40:1059-66.

10. Zambon A, Puato M, Faggin E, et al. Lipoprotein remnants and dense LDL are associated with features of unstable carotid plaque: a flag for non-HDL-C. Atherosclerosis 2013;230:106-9.

11. Hansson GK. Inflammation, atherosclerosis, and coronary artery disease. N Engl J Med 2005;352:1685-95.

12. Ramkhelawon B, Yang Y, van Gils JM, et al. Hypoxia induces netrin-1 and Unc5b in atherosclerotic plaques: mechanism for macrophage retention and survival. Arterioscler Thromb Vasc Biol 2013;33:1180-8.

13. Zanellato AM, Borrione AC, Tonello M, et al. Myosin isoform expression and smooth muscle cell heterogeneity in normal and atherosclerotic rabbit aorta. Arteriosclerosis 1990;10:996-1009.

14. Giuriato L, Chiavegato A, Pauletto P, Sartore S. Correlation between the presence of an immature smooth muscle cell population in tunica media and the development of atherosclerotic lesion. A study on different-sized rabbit arteries from cholesterol-fed and Watanabe heritable hyperlipemic rabbits. Atherosclerosis 1995;116:77-92.

15. Sartore S, Chiavegato A, Faggin E, et al. Contribution of adventitial fibroblasts to neointima formation and vascular remodeling: from innocent bystander to active participant. Circ Res 2001;89:1111-21.

16. Geng YJ, Libby P. Progression of atheroma: a struggle between death and procreation. Arterioscler Thromb Vasc Biol 2002;22:1370-80.

17. Ross R. Atherosclerosis is an inflammatory disease. Am Heart J 1999;138:S419-20.

18. Libby P. Inflammation in atherosclerosis. Arterioscler Thromb Vasc Biol 2012;32:2045-51.

19. Rattazzi M, Puato M, Faggin E, et al. C-reactive protein and interleukin-6 in vascular disease: culprits or passive bystanders? J Hypertens 2003;21:1787-803.

20. Rattazzi M, Faggin E, Bertipaglia B, Pauletto P. Innate immunity and atherogenesis. Lupus 2005;14:747-51.

21. Latini R, Maggioni AP, Peri G, et al. Prognostic significance of the long pentraxin PTX3 in acute myocardial infarction. Circulation 2004;110:2349-54.

22. Gertz SD, Roberts WC. Hemodynamic shear force in rupture of coronary arterial atherosclerotic plaques. Am J Cardiol 1990;66:1368-72.
23. Mintz GS, Pichard AD, Popma JJ, et al. Determinants and correlates of target lesion calcium in coronary artery disease: a clinical, angiographic and intravascular ultrasound study. J Am Coll Cardiol 1997;29:268-74.

24. Wahlgren CM, Zheng W, Shaalan W, et al. Human carotid plaque calcification and vulnerability. Relationship between degree of plaque calcification, fibrous cap inflammatory gene expression and symptomatology. Cerebrovasc Dis 2009;27:193-200.

25. Giachelli CM. Vascular calcification mechanisms. J Am Soc Nephrol 2004;15:2959-64.

26. Price PA, Nguyen TM, Williamson MK. Biochemical characterization of the serum fetuin-mineral complex. J Biol Chem 2003;278:22153-60.

27. Price PA, Caputo JM, Williamson MK. Bone origin of the serum complex of calcium, phosphate, fetuin, and matrix Gla protein: biochemical evidence for the cancellous bone-remodeling compartment. J Bone Miner Res 2002;17:1171-9.

28. Buso G, Faggin E, Pauletto P, Rattazzi M. Osteoprotegerin in cardiovascular disease: ally or enemy? Curr Pharm Des 2014;20:5862-9.

29. Rattazzi M, Bennett BJ, Bea F, et al. Calcification of advanced atherosclerotic lesions in the innominate arteries of ApoE-deficient mice: potential role of chondrocyte-like cells. Arterioscler Thromb Vasc Biol 2005; 25:1420-5.

30. Rattazzi M, Iop L, Faggin E, et al. Clones of interstitial cells from bovine aortic valve exhibit different calcifying potential when exposed to endotoxin and phosphate. Arterioscler Thromb Vasc Biol 2008;28:2165-72.

31. Tintut Y, Patel J, Parhami F, Demer LL. Tumor necrosis factor-alpha promotes in vitro calcification of vascular cells via the cAMP pathway. Circulation 2000;102: 2636-42.

32. Sakakura K, Nakano M, Otsuka F, et al. Pathophysiology of atherosclerosis plaque progression. Heart Lung Circ 2013;22:399-411.

33. Finn AV, Nakano M, Narula J, et al. Concept of vulnerable/unstable plaque. Arterioscler Thromb Vasc Biol 2010;30:1282-92.

34. Pauletto P, Puato M, Faggin E, et al. Specific cellular features of atheroma associated with development of neointima after carotid endarterectomy: the carotid atherosclerosis and restenosis study. Circulation 2000;102: 771-8.

35. Kramer MC, Rittersma SZ, de Winter RJ, et al. Relationship of thrombus healing to underlying plaque morphology in sudden coronary death. J Am Coll Cardiol 2010;55:122-32.

36. Moreno PR, Bernardi VH, Lopez-Cuellar J, et al. Macrophage infiltration predicts restenosis after coronary intervention in patients with unstable angina. Circulation 1996;94:3098-102.

37. Hellings WE, Moll FL, De Vries JP, et al. Atherosclerotic plaque composition and occurrence of restenosis after carotid endarterectomy. JAMA 2008;299:547-54.

38. Puato M, Zambon A, Faggin E, et al. Statin treatment and carotid plaque composition: a review of clinical studies. Curr Vasc Pharmacol 2014;12:518-26.

39. Crisby M, Nordin-Fredriksson G, Shah PK, et al. Pravastatin treatment increases collagen content and decreases lipid content, inflammation, 
metalloproteinases, and cell death in human carotid plaques: implications for plaque stabilization. Circulation 2001;103:926-33.

40. Cortellaro M, Cofrancesco E, Arbustini E, et al. Atorvastatin and thrombogenicity of the carotid atherosclerotic plaque: the ATROCAP study. Thromb Haemost 2002; 88:41-7.

41. Martin-Ventura JL, Blanco-Colio LM, Gomez-Hernandez A, et al. Intensive treatment with atorvastatin reduces inflammation in mononuclear cells and human atheroscle- rotic lesions in one month. Stroke 2005;36: 1796-800.

42. Puato M, Faggin E, Rattazzi M, et al. Atorvastatin reduces macrophage accumulation in atherosclerotic plaques: a comparison of a nonstatin-based regimen in patients undergoing carotid endarterectomy. Stroke 2010;41: 1163-8.

43. Nissen SE, Tuzcu EM, Schoenhagen P, et al. Effect of intensive compared with moderate lipid-lowering therapy on progression of coronary atherosclerosis: a randomized controlled trial. JAMA 2004;291:1071-80. 


\title{
La prevenzione secondaria nella cardiopatia ischemica
}

\author{
Domenico Panuccio, ${ }^{1}$ Paolo Verdecchia ${ }^{2}$ \\ ${ }^{1}$ UOC Medicina Interna, Ospedale Maggiore, Bologna; ${ }^{2}$ UOC Medicina, Ospedale di Assisi, Assisi, Italia
}

\section{Premessa}

La Federazione delle Associazioni dei Dirigenti Ospedalieri Internisti (FADOI), con riferimento alla cardiopatia ischemica, intende focalizzare l'attenzione sulla prevenzione cardiovascolare secondaria, definita come prevenzione di un futuro evento cardiovascolare maggiore in pazienti a rischio cardiovascolare molto elevato (very high risk) $)^{1}$ in relazione alla presenza di cardiopatia ischemica manifesta, documentata da una o più delle seguenti condizioni: i) precedente diagnosi di sindrome coronarica acuta (angina instabile elo infarto miocardico con o senza sopra-slivellamento del tratto ST); ii) precedente diagnosi di ischemia miocardica (coronarografia, ergometria, scintigrafia, ecocardiografia da stress); iii) precedente esecuzione di by-pass aorto coronarico o angioplastica coronarica.

\section{Valutazione del rischio e stratificazione prognostica}

La European Society of Cardiology (ESC) ha ritenuto di accomunare a questi pazienti, nella definizione di rischio cardiovascolare molto elevato, anche i pazienti con precedente diagnosi di ictus cerebrale, ovvero di precedente diagnosi di arteriopatia periferica occlusiva, placche carotidee, diabete mellito tipo $1 \mathrm{o}$ 2 purchè in associazione con altri fattori di rischio oppure microalbuminuria (30-300 mg/24 ore), nefropatia cronica (velocità di filtrazione glomerulare $<30$ $\mathrm{mL} / \mathrm{min}$; rischio di mortalità a 10 anni $\geq 10 \%$ secondo la carta del rischio SCORE). ${ }^{1}$ Questo capitolo tratterà,

Corrispondente: Paolo Verdecchia, Ospedale di Assisi, Struttura Complessa di Medicina, via Valentin Müller 1, 06081 Assisi, Italia.

Fax: +39.075.8139301

E-mail: verdec@tin.it

Articolo pubblicato secondo la Creative Commons Attribution NonCommercial 3.0 License (CC BY-NC 3.0).

CCopyright D. Panuccio e P. Verdecchia, 2015

Licensee PAGEPress, Italy

QUADERNI - Italian Journal of Medicine 2015; 3:328-340 tuttavia, solo i pazienti con cardiopatia ischemica, anche in rapporto ad alcune co-morbilità molto frequenti nei reparti di medicina e che non vengono trattate in capitoli specifici.

\section{Entità del problema}

Le malattie cardiovascolari rappresentano la prima causa di morte a livello mondiale sia nel genere maschile che in quello femminile. Sebbene la mortalità cardiovascolare stia progressivamente e lentamente declinando nei paesi occidentali, la sua tendenza è invece in aumento nell'Europa dell'Est. Oltre il 50\% della riduzione della mortalità per cardiopatia ischemica è imputabile alla riduzione dei fattori di rischio, mentre la restante porzione è imputabile a trattamenti farmacologici e non farmacologici sempre più appropriati. ${ }^{1,2}$ Purtroppo, lo studio EOROASPIRE III, eseguito in 8966 pazienti reclutati in 22 Paesi europei, ha evidenziato che solo una piccola parte dei soggetti mostra un effettivo e soddisfacente controllo dei fattori di rischio cardiovascolare $^{1-3}$

\section{Raccomandazioni in pazienti con cardiopatia ischemica cronica}

\section{Strategie educative/comportamentali}

I pazienti con cardiopatia ischemica cronica possono beneficiare di programmi educazionali nei quali medici, infermieri e psicologi hanno l'opportunità di interagire con i pazienti stessi, ed i pazienti l'opportunità di interagire tra di loro, sui fattori di rischio, sulle modificazioni dello stile di vita e su una maggiore conoscenza delle terapie. Nonostante una larga parte della riduzione della mortalità per cardiopatia ischemica (tra il $44 \%$ ed il 76\%) sia attribuibile alle modificazioni dei fattori di rischio (riduzione fumo di sigaretta, riduzione pressione arteriosa, etc.), questi sono effettivamente sotto controllo (a goal) solo in una bassa percentuale (tra il $12 \%$ ed il $55 \%$ ) di cittadini europei. ${ }^{4-57}$ I risultati di varie metanalisi supportano questi provvedimenti, seppure in proporzioni e sotto aspetti diversi. ${ }^{4,5,26}$

Le 10 regole pratiche da seguire in questo tipo di strategie sono le seguenti: ${ }^{1}$ i) sviluppare un'alleanza 
anche di tipo psicologico con il paziente; ii) implementare tali strategie in tutti i pazienti con malattia cardiovascolare manifesta o ad alto rischio; iii) far comprendere ai pazienti i rapporti tra corretto stile di vita e salute/benessere; iv) aiutare i pazienti a comprendere le barriere che ostacolano le modificazioni comportamentali; v) motivare i pazienti perché modifichino gli stili di vita; vi) coinvolgere i pazienti nell'identificazione dei principali fattori di rischio da modificare; vii) impiegare varie strategie per rinforzare le capacità individuali a cambiare; viii) formalizzare un piano preciso di modificazione degli stili di vita; ix) coinvolgere altre figure professionali; $\mathrm{x}$ ) monitorare i progressi attraverso un attento follow-up.

\section{Fumo di sigaretta}

È ben noto che il fumo di sigaretta aumenta il rischio di morbilità e mortalità cardiovascolare in relazione diretta con l'esposizione e la durata dell'abitudine al fumo, ed indipendentemente dal fatto che si tratti di sigarette, sigari, pipa o sigarette con filtri particolari oppure che si tratti di fumo attivo o passivo. ${ }^{1}$ È altrettanto noto che la sospensione del fumo di sigaretta si associa ad una rapido miglioramento della prognosi. Una meta-analisi di studi eseguiti in prevenzione secondaria in pazienti sopravvissuti ad un infarto miocardico acuto, ha mostrato che la sospensione del fumo di sigaretta si accompagna ad una riduzione della mortalità del $36 \%$, indipendentemente da qualsiasi altro fattore confondente. ${ }^{58} \mathrm{Il}$ beneficio prognostico della sospensione del fumo di sigaretta appare già evidente a 6 mesi dopo l'evento acuto, sebbene occorrano 10-15 anni perché il rischio di eventi cardiovascolari divenga sovrapponibile a quello dei non fumatori. ${ }^{56} \mathrm{La}$ semplice riduzione quantitativa (senza sospensione completa) del fumo di sigaretta non dovrebbe essere mai consigliata, per la mancanza di dati credibili sulla sua efficacia. Qualsiasi esposizione al fumo attivo o passivo dovrebbe essere evitata sia in prevenzione secondaria che in prevenzione primaria (Raccomandazione di classe I, livello di evidenza B). A tutti i fumatori andrebbe offerta assistenza strutturata e qualificata per facilitare la cessazione completa del fumo, all'interno di programmi specifici (Raccomandazione di classe I, livello di evidenza A). ${ }^{59}$

\section{Dieta}

Una dieta sana, fondamentale per la prevenzione delle malattie cardiovascolari (Raccomandazione di classe I, livello di evidenza B), dovrebbe avere le seguenti caratteristiche: ${ }^{1}$ i) intake energetico totale determinato dagli acidi grassi saturi in ragione inferiore al 10\% (per la restante quota determinato dagli acidi grassi poli-insaturi); ii) meno di $5 \mathrm{~g}$ di sale per giorno; iii) almeno 30-45 g di fibre per giorno (frutta, verdura, cereali); iv) almeno $200 \mathrm{~g}$ di frutta e $200 \mathrm{~g}$ di vegetali per giorno (in 2-3 portate); v) un pasto di pesce almeno 2 volte per settimana; vi) non oltre due bicchieri di vino al giorno nei maschi ( $20 \mathrm{~g} /$ die di alcool) e non oltre 1 bicchiere di vino al giorno nelle femmine (10 $\mathrm{g}$ /die di alcool); vii) l'intake energetico dovrebbe essere sufficiente a mantenere un indice di massa corporea $<25 \mathrm{~kg} / \mathrm{m}^{2}$ di superficie corporea.

\section{Grassi saturi}

Il rischio di cardiopatia ischemica decresce del 2$3 \%$ per ogni $1 \%$ di intake energetico trasferito da grassi saturi a grassi polinsaturi. ${ }^{13}$

\section{Sale}

Una riduzione dell'apporto di sale da cucina di solo $1 \mathrm{~g}$ /die (corrispondente a $0,4 \mathrm{~g}$ di $\mathrm{Na}$ e $0,6 \mathrm{~g}$ di cloro) si accompagna ad una riduzione della pressione arteriosa di 3,1 mmHg. ${ }^{60} \mathrm{Si}$ sa che l'organismo umano contiene circa $110 \mathrm{~g}$ di sale e ne elimina giornalmente 0,25-1,52 g. Purtroppo, l'introito medio giornaliero di sale in Italia (ed Europa) è di circa $10 \mathrm{~g}$. Pertanto, l'introito giornaliero di sale andrebbe praticamente dimezzato. Una riduzione dell'apporto di sale di $3 \mathrm{~g} / \mathrm{die}$ potrebbe portare ad una riduzione della cardiopatia ischemica del 6-9\%, una riduzione dell'ictus del 5-8\% ed una riduzione della mortalità totale del $3-4 \%{ }^{61}$

\section{Pesce}

Un modesto incremento del consumo di pesce a 12 porzioni per settimana ridurrebbe l'incidenza della mortalità per cardiopatia ischemica del $36 \%{ }^{62}$

\section{Vino}

Per quanto riguarda il vino, in particolare quello rosso, ricco di polifenoli, è stato stimato che l'intake ottimale ai fini della riduzione della mortalità da tutte le cause è pari a $20 \mathrm{~g} / \mathrm{die}$ di alcool nei maschi e 10 $\mathrm{g} /$ die nelle femmine. ${ }^{14}$

\section{Attività fisica}

I pazienti con cardiopatia ischemica cronica accertata dovrebbero svolgere sedute di attività fisica almeno 3 volte per settimana, con durata di almeno 30 min per seduta: per tale obiettivo i pazienti sedentari dovrebbero essere incoraggiati ad iniziare un'attività fisica lieve dopo adeguata valutazione clinica di base (Raccomandazione di classe I, livello di evidenza A). ${ }^{63}$

Una meta-analisi di studi eseguiti in pazienti affetti in larga parte da cardiopatia ischemica cronica ha mostrato che un programma di esercizio fisico continuativo per almeno 3 mesi si accompagnava ad una riduzione del $30 \%$ della mortalità cardiovascolare, e del $35 \%$ della mortalità per cause coronariche. ${ }^{64,65}$ 


\section{Peso corporeo}

In generale, la riduzione del peso corporeo nei soggetti obesi (indice di massa corporea [IMC] $>30,0$ $\mathrm{kg} /$ altezza $[\mathrm{m}]^{2}$ ) o sovrappeso (IMC tra 25,0 e 29,9 $\mathrm{kg} /$ altezza $[\mathrm{m}]^{2}$ ) continua ad essere fortemente raccomandata, in quanto associata ad una riduzione della pressione arteriosa e della dislipidemia, con conseguente riduzione del rischio cardiovascolare (Raccomandazione di classe I, livello di evidenza A). ${ }^{1}$ Tuttavia, nei pazienti con cardiopatia ischemica cronica le evidenze sono piuttosto contraddittorie poiché alcuni studi hanno mostrato una sorta di effetto protettivo esercitato dall'obesità sul rischio cardiovascolare in prevenzione secondaria. ${ }^{66-68}$

\section{Pressione arteriosa}

Il trattamento antiipertensivo deve essere iniziato senza indugio in pazienti ipertesi con diagnosi di cardiopatia ischemica cronica (o altri eventi cardiovascolari maggiori) ed ipertensione in stadio I o II, alla stregua dei pazienti con ipertensione in stadio III (Raccomandazione di classe I, livello di evidenza C). Negli altri pazienti è consigliabile un periodo di attesa di qualche settimana o qualche mese, cercando di attuare misure dietetiche/comportamentali. ${ }^{1,30}$ L'orientamento attuale delle linee guida Europee è quello di ridurre la pressione arteriosa sistolica a valori inferiori ai 140 $\mathrm{mmHg}$, e la diastolica a valori inferiori ai $90 \mathrm{mmHg}$ in tutti i pazienti ipertesi, indipendentemente dall'anamnesi positiva o negativa per evento cardiovascolare maggiore (Raccomandazione di classe IIa, livello di evidenza A). ${ }^{23-25}$ Nei pazienti ultraottantenni, l'obbiettivo di una riduzione della pressione arteriosa sistolica al di sotto dei $150 \mathrm{mmHg}$ appare accettabile. ${ }^{69}$ Alcuni autori hanno anche sollevato la possibilità di una curva $J$, ovvero di un aumento paradosso del rischio cardiovascolare nei pazienti nei quali si raggiungevano valori pressori più bassi. ${ }^{70}$ Tuttavia, alcune analisi di meta-regressione e un'analisi specifica del mega-trial ONTARGET ristretta ai pazienti con diagnosi accertata di cardiopatia ischemica cronica hanno dimostrato che il raggiungimento di un target pressorio $<130 / 80 \mathrm{mmHg}$ in pazienti ad alto rischio cardiovascolare si associa ad una drammatica riduzione del rischio di ictus cerebrale senza alcun incremento $p a$ radosso del rischio di infarto miocardico. ${ }^{71}$ Quando presente, tale aumento paradosso sembra attribuibile a patologie concomitanti (cancro, scompenso cardiaco, epatopatie, ecc.) in grado sia di ridurre la pressione arteriosa, sia di peggiorare, per effetto proprio ed indipendente, la prognosi del paziente. L'estremo sinistro della curva $\mathrm{J}$ non sarebbe dunque colpa del trattamento antiipertensivo, ma di patologie concomitanti con effetto confondente. ${ }^{71}$ Quali farmaci impiegare preferenzialmente? L'orientamento delle linee guida Europee è che diuretici, ACE-inibitori, sartani, $\mathrm{Ca}^{++}$antagonisti e $\beta$-bloccanti non differiscono tra loro in termini di efficacia antiipertensiva. Quindi, queste 5 classi di farmaci possono essere considerate di pari valore per l'inizio ed il mantenimento della terapia (Raccomandazione di classe I, livello di evidenza A). ${ }^{1,72}$ Le stesse linee guida consigliano alcuni farmaci rispetto ad altri in base a condizioni cliniche e patologie associate.

\section{Colesterolo}

È ormai ampiamente accettato che: i) nei pazienti ospedalizzati per sindrome coronarica acuta la statina debba essere iniziata già durante il ricovero; ii) in tutti i pazienti con diagnosi di cardiopatia ischemica cronica o altro evento cardiovascolare maggiore (così come nei pazienti con pregresso ictus non trombo-embolico e nei pazienti con arteriopatia occlusiva periferica) la statina debba essere iniziata prontamente, con l'obiettivo di ridurre la colesterolemia con lipoproteine a bassa densità (c-LDL) a valori inferiori a 70 $\mathrm{mg} / \mathrm{dL}$ (Raccomandazione di classe I, livello di evidenza A): diverse meta-analisi hanno dimostrato che la riduzione del c-LDL a valori inferiori a $70 \mathrm{mg} / \mathrm{dL}$ si associa ai più bassi livelli di mortalità in pazienti con cardiopatia ischemica cronica. ${ }^{63}$

In pazienti con cardiopatia ischemica cronica, un trattamento con statine riduce non solo il rischio di infarto miocardico ricorrente, ma anche quello di ictus cerebrale..$^{41}$ Come è noto, le statine possono indurre un incremento, generalmente reversibile, degli enzimi epatici nel $5-10 \%$ dei pazienti, ma la rabdomiolisi vera e propria è estremamente rara. Purtroppo, solo una bassa percentuale $(<50 \%)$ di pazienti trattati con statine raggiunge i target lipidici raccomandati. ${ }^{73}$ Allo scopo di massimizzare l'efficacia delle statine una volta raggiunta la loro massima dose tollerata, queste possono essere combinate con farmaci sequestratori di acidi biliari, oppure con ezetimibe, che permette di raggiungere analoghi target lipidici a dosi più basse di statine di quelle che sarebbero necessarie utilizzando le statine in monoterapia. ${ }^{74}$

Recentemente, lo studio IMPROVE-IT, eseguito in pazienti con pregressa sindrome coronarica acuta e randomizzati ad un trattamento con simvastatina oppure con simvastatina+ezetimibe per un periodo di 7 anni, ha dimostrato una riduzione dell'end-point primario (composito di infarto miocardico, angina instabile richiedente rivascolarizzazione o ictus) del 6,4\% $(\mathrm{P}=0,016)$. La riduzione assoluta del rischio è stata del $2,0 \%$ (end-point primario registrato nel $32,7 \%$ dei pazienti trattati con la combinazione e nel $34,7 \%$ dei pazienti trattati con simvastatina da sola).

Mancano ancora vaste evidenze su una nuova classe di farmaci anticolesterolemici basata sull'impiego di anticorpi monoclonali anti PSK9 (proproteina 
convertasi subtilisina/kexina tipo 9), ma dati disponibili su Alirocumab e con Evolocumab hanno dimostrato che aggiunti alla terapia standard (statine o ezetimibe) hanno ridotto il c-LDL fino al 70\% e la lipoproteina (a) intorno al $32 \% .^{75,76}$

\section{Antitrombotici}

Come è noto, l'aspirina non deve essere somministrata in prevenzione primaria, neppure in soggetti ad alto rischio, perché il rischio di sanguinamenti, anche gravi, è superiore al beneficio atteso in termini antitrombotici. ${ }^{36}$

$\mathrm{Al}$ contrario, dopo una sindrome coronarica acuta, indipendentemente dal fatto che il paziente abbia eseguito procedure di rivascolarizzazione e/o abbia applicato stent, l'aspirina è indicata per una durata di 12 mesi in associazione con un agente inibitore dei recettori P2Y12 (ticagrelor o prasugrel) purché tale associazione non sia controindicata per eccessivo rischio di sanguinamento. ${ }^{77} \mathrm{Nei}$ pazienti che non possono ricevere ticagrelor o prasugrel è indicato il clopidogrel (600 $\mathrm{mg}$ di carico, $75 \mathrm{mg} /$ die successivamente) assieme all'aspirina. Dopo il $12^{\circ}$ mese, l'aspirina da sola deve essere somministrata a tempo indeterminato (Raccomandazione di classe I, livello di evidenza A). La raccomandazione di somministrare aspirina a tempo indeterminato è basata sui risultati di una ben nota metanalisi di studi eseguiti in 17.000 pazienti con precedente infarto miocardico, ictus cerebrale o diagnosi di arteriopatia ostruttiva, che ha dimostrato una riduzione dell'incidenza di eventi cardiovascolari maggiori nel lungo termine ad opera dell'aspirina dall' 8,2 al $6,7 \%$. In quest'analisi, l'aspirina ha anche ridotto significativamente, del 10\%, la mortalità da tutte le cause. ${ }^{36}$ La scelta tra l'aspirina ed il clopidogrel in monoterapia nel lungo termine potrebbe essere orientata dai risultati dello studio CAPRIE, che ha dimostrato che, in pazienti con pregresso infarto, ictus o arteriopatia occlusiva, l'incidenza di eventi maggiori è stata del 5,83\% per anno con aspirina, e del 5,32\% per anno con clopidogrel $(\mathrm{P}=0,043) .^{78}$

\section{Glicemia}

Sia in prevenzione secondaria che in primaria, l'HbAlc dovrebbe essere ridotta a livelli $<7,0 \%$ nei pazienti diabetici (Raccomandazione di classe I, livello di evidenza $A$ ), utilizzando la metformina come farmaco di prima scelta, se non controindicata (Raccomandazione di classe IIa, livello di evidenza B). ${ }^{1} \mathrm{E}$ necessario evitare tanto l'ipoglicemia, ${ }^{79}$ quanto l'incremento ponderale (Raccomandazione di classe I, livello di evidenza B) e tale raccomandazione è particolarmente valida in pazienti con cardiopatia ischemica cronica, che potrebbero risentire in maniera molto negativa di episodi di ipoglicemia. ${ }^{27,55}$

\section{Pazienti con cardiopatia ischemica cronica e altre comorbidità}

I pazienti di età $>45$ anni affetti da cardiopatia ischemica nota presentano frequentemente patologie croniche concomitanti che possono condizionare direttamente o indirettamente il trattamento della patologia coronarica: artrite/artrosi nel 56,7\%, deterioramento cognitivo nel $29,9 \%$, scompenso cardiaco nel $29 \%$, broncopneumopatia cronica ostruttiva (BPCO) nel $25,5 \%$, diabete mellito nel $24,8 \%$, turbe psichiche nel $14,3 \%$, esiti di stroke nel $13,8 \%$, anemia nel $10,1 \%$, disturbi della vista $(16,7 \%)$ o dell'udito $(17,9 \%) .{ }^{80} \mathrm{Al}-$ cune di queste comorbidità saranno oggetto di specifici paragrafi di approfondimento ai quali si rimanda. In questa sede riteniamo doveroso fare cenno, seppur breve, alle problematiche relative ad alcune comorbidità (artrosi, BPCO, depressione) che più di altre possono condizionare l'applicazione delle linee guida della prevenzione cardiovascolare secondaria o che possono interferire pesantemente sulla prognosi.

\section{Prevenzione cardiovascolare secondaria nei pazienti con artrite/artrosi}

Le artropatie croniche (artrosi ed artrite reumatoide) sono le comorbidità più frequenti nei soggetti con cardiopatia ischemica ${ }^{80}$ Sia l'artrosi ${ }^{81}$ che l'artrite reumatoide $(\mathrm{AR})^{82}$ sembrano avere legami di tipo fisiopatologico con la cardiopatia ischemica basati sull'infiammazione sistemica cronica e su uno stato protrombotico, ${ }^{83}$ ed espongono i pazienti ad un aumentato rischio di incidenza di malattia coronarica. Tale rischio può anche precedere nel tempo l'esordio clinico dell'AR, che è indipendente dai tradizionali fattori di rischio cardiovascolare ${ }^{82}$ e che, nella fase di malattia conclamata, è correlata all'intensità biologica della malattia stessa. ${ }^{84} \mathrm{La}$ mortalità nei pazienti con $\mathrm{AR}$ è di tre volte più elevata rispetto alla popolazione generale $\mathrm{e}$ nel $40 \%$ dei casi è dovuta a cause cardiovascolari. ${ }^{85} \mathrm{Il}$ trattamento di fondo dell'AR con farmaci immunosoppressivi (metotrexato) e con i più recenti farmaci biologici (inibitori dell'interleuchina 6) sembra avere effetti benefici anche su marcatori biologici ${ }^{86}$ ed emodinamici delle malattie cardiovascolari. ${ }^{87}$

\section{Il problema dei farmaci anti-infiammatori non steroidei}

La prevenzione coronarica secondaria nei pazienti affetti anche da artropatie croniche può essere influenzata in maniera importante dai farmaci anti-infiammatori non steroidei di cui molti pazienti fanno uso prolungato. Numerose ricerche, in prevalenza di tipo osservazionale, dimostrano una chiara correlazione tra uso di farmaci anti-infiammatori non steroidei (FANS) ed incidenza di eventi cardiovascolari, in popolazioni non selezionate. In maniera semplicistica possiamo riassumere questa 
correlazione in due concetti semplici: i) tutti i FANS possono aumentare l'incidenza di eventi cardiovascolari; ii) i FANS selettivi per le COX-2 (Coxib) comportano un rischio più elevato rispetto ai non selettivi.

Nell'ambito di entrambe queste due categorie di farmaci si può riscontrare una certa graduazione del rischio tra le varie molecole. Un'ampia meta-analisi pubblicata di recente ${ }^{88}$ ha calcolato che nei pazienti ad elevato rischio cardiovascolare l'uso di Coxib o di alte dosi di diclofenac o ibuprofene può comportare incidenza di 7-8 eventi cardiovascolari maggiori (di cui due fatali) ogni 1000 soggetti trattati per anno; mentre per il naprossene (pur con qualche riserva) sembra che questa associazione sia meno significativa. Il tipo di infarto più frequentemente correlato all'uso di FANS è quello senza sopraslivellamento del tratto ST (NSTEMI), ${ }^{89}$ che spesso si manifesta con caratteristiche atipiche e che pertanto più facilmente può essere misconosciuto.

Il rischio di mortalità e recidiva di infarto correlati all'uso dei FANS si verifica anche dopo solo pochissimi giorni di terapia e persiste elevato per tutto il periodo di trattamento, ${ }^{90}$ indipendentemente dal tempo trascorso dal primo evento coronarico. Entro 12 mesi da un primo infarto miocardico si verifica recidiva infartuale o morte coronarica nel $18 \%$ dei pazienti che fanno uso di FANS rispetto al $6 \%$ circa per quelli che non ne fanno uso. Anche a distanza di cinque o più anni il rischio coronarico correlato ai FANS si mantiene significativamente più elevato. ${ }^{91}$ Questi dati indicano che nei pazienti con pregresso infarto miocardico acuto non esiste una finestra terapeutica sicura per l'uso di FANS, in termini di tempo trascorso dall'evento coronarico, né in termini di durata del trattamento stesso.

In conclusione, mentre i farmaci per la terapia di fondo dell'AR (immunosoppressori e biologici) non hanno effetti negativi in ambito cardiovascolare, anzi migliorano alcuni end point surrogati, la terapia sintomatica dell'AR e dell'artrosi con FANS può avere un impatto negativo nei pazienti con pregresso infarto miocardico in quanto questi farmaci favoriscono le recidive infartuali ed aumentano la mortalità cardiovascolare. Pertanto i FANS vanno utilizzati con molta cautela o possibilmente evitati nei pazienti con pregresso evento coronarico acuto.

\section{Prevenzione cardiovascolare secondaria nei pazienti con broncopneumopatia cronica ostruttiva}

Cardiopatia ischemica e BPCO sono condizioni patologiche frequentemente presenti nello stesso paziente. Circa il $20 \%$ dei pazienti con BPCO sono affetti anche da patologie cardiovascolari ${ }^{92}$ e questa associazione comporta una prognosi peggiore in termini di aumentata mortalità,${ }^{93}$ indipendentemente da altri fattori di rischio come genere ed età. ${ }^{44}$ In tutti gli stadi di gravità della BPCO la principale causa di mor- talità è rappresentata proprio dalle malattie cardiovascolari, responsabili del 20-30\% dei decessi. ${ }^{95} \mathrm{Il}$ fumo è riconosciuto come importante fattore di rischio per entrambe queste patologie e la sua rimozione è mandatoria nella terapia e nella prevenzione di ciascuna di esse. Nella prevenzione cardiovascolare secondaria nei pazienti con BPCO va ribadito che le raccomandazioni generali delle linee guida relative allo stile di vita (abolizione del fumo, riduzione del sovrappeso corporeo, dieta equilibrata, attività fisica), così come la correzione dei vari fattori di rischio, vanno mantenute anche in questi pazienti.

Troppo spesso la BPCO condiziona una minore aderenza alle linee guida, ma bisogna riconoscere che le problematiche dei pazienti con BPCO e cardiopatia ischemica sono in genere poco affrontate nelle linee guida, con la sporadica eccezione dei $\beta$-bloccanti.

\section{$\beta$-bloccanti, inibitori del sistema renina angiotensina, statine}

I $\beta$-bloccanti sono farmaci di straordinaria importanza nei pazienti con pregresso infarto miocardico o con scompenso cardiaco. Entrambe queste patologie sono frequentemente presenti nei pazienti con BPCO nei quali per lungo tempo si è ritenuto che i $\beta$-bloccanti fossero controindicati, tanto da rappresentare un criterio di esclusione dai grandi trial. Oggi è invece accertato che nei pazienti con BPCO con associata una patologia cardiovascolare per la quale ne è raccomandato l'uso, i $\beta$-bloccanti cardioselettivi comportano benefici anche alla patologia respiratoria in termini di una minore incidenza di riacutizzazioni ${ }^{96}$ e di mortalità nel lungo periodo. ${ }^{97} \grave{E}$ anche dimostrato che l'uso contemporaneo di $\beta$-bloccanti e $\beta 2$-agonisti è sicuro ed ha un impatto positivo sulla prognosi. ${ }^{98}$

Anche altri farmaci utilizzati nella prevenzione cardiovascolare secondaria, come gli ACE-inibitori, i sartani e le statine hanno dimostrato effetti positivi. ${ }^{99-103}$

\section{$\beta$-stimolanti e anticolinergici}

Un tema molto dibattuto in letteratura è se i farmaci respiratori, in particolare $\beta$-stimolanti ed anticolinergici, utilizzati per la terapia cronica della BPCO possano avere effetti negativi a livello cardiovascolare. I $\beta$-stimolanti possono causare, soprattutto nelle fasi iniziali di trattamento e con le formulazioni a breve durata d'azione, aumento della frequenza cardiaca e sono stati associati ad una maggiore incidenza di aritmie ipercinetiche compresa la fibrillazione atriale, ${ }^{104}$ ma non sono stati riscontrati effetti negativi sulla prognosi cardiovascolare. Per i farmaci anticolinergici una metanalisi di qualche anno fa evidenziava un aumentato rischio di infarto miocardico e morte cardiovascolare che era statisticamente significativo per quelli a più breve durata d'azione, come l'ipratropio, e solo tendenziale per quelli a lunga durata d'azione come il tio- 
tropio. ${ }^{105}$ Un successivo studio randomizzato e controllato documentava una riduzione non significativa della mortalità per tutte le cause e, in un'analisi post hoc, una significativa riduzione della mortalità cardiovascolare e dell'infarto miocardico nei pazienti con BPCO trattati con tiotropio rispetto al placebo. ${ }^{106}$

In conclusione, si può ragionevolmente ritenere che nei pazienti con coronaropatia e BPCO tutti i provvedimenti, farmacologici e non farmacologici, necessari per la prevenzione secondaria della prima e per il trattamento della seconda vanno utilizzati senza remore, anzi con la consapevolezza che oltre ad esplicare i benefici effetti sulle rispettive patologie ne esplicano anche altri sulla comorbidità associata.

\section{Prevenzione cardiovascolare secondaria nei pazienti con depressione}

Circa il 20\% dei pazienti con cardiopatia ischemica ha sintomi depressivi o una forma conclamata di depressione. ${ }^{107}$ Seppure i dati sull'argomento siano molto eterogenei, una recente ampia metanalisi commissionata dall'American Heart Association (AHA) ha concluso che le evidenze disponibili hanno forza sufficiente per considerare la depressione un fattore di rischio per una prognosi peggiore nei pazienti sopravvissuti ad un evento coronarico acuto. ${ }^{108} \mathrm{La}$ depressione ha una correlazione con la cardiopatia ischemica non solo nei pazienti con precedente evento coronarico acuto, ma, come ha dimostrato lo studio INTERHEART, ${ }^{109}$ aumenta del $56 \%$ il rischio di un primo episodio di infarto miocardico acuto in maniera indipendente da altri fattori di rischio come età, genere e fumo. I meccanismi fisiopatologici che legano queste due patologie sono molteplici, ma schematicamente riconducibili a due categorie: interferenza con alcuni fattori di rischio cardiovascolare tradizionali (diabete tipo $2,{ }^{110}$ ipertensione, ${ }^{111}$ obesità $^{112}$ ) ed alterazioni neuro-endocrine. Le alterazioni neuroendocrine individuate sono complesse e numerose, ma certamente l'iperattività dell'asse ipotalamoipofisi-surrene è tra le più documentate ed importanti. Un'altra risposta allo stress cronico dovuto alla depressione è lo squilibrio del sistema nervoso autonomo con attivazione della componente adrenergica e conseguenti multipli effetti pro-trombotici nel sistema cardiovascolare. ${ }^{113}$ Le strette correlazioni epidemiologiche e fisiopatologiche, prima brevemente descritte, che danno robuste argomentazioni per considerare la depressione come fattore di rischio cardiovascolare, generano la logica aspettativa che il trattamento della depressione comporti una riduzione degli eventi cardiovascolari. Per quanto riguarda i pazienti con precedente evento coronarico, alcuni studi hanno cercato di valutare se il trattamento della depressione potesse ridurre l'incidenza di recidive o di mortalità. Una meta-analisi ${ }^{114}$ che ha analizzato i dati di tutti questi studi ha concluso che non vi sono sufficienti evidenze per affermare che il trattamento farmacologico e/o psicoterapeutico comporti una riduzione degli eventi coronarici statisticamente significativa, riscontrando solo un beneficio tendenziale. Va segnalato che alcuni di questi studi non erano scevri da problemi di tipo metodologico, ma anche lo studio ENRICHD, che più specificamente era stato disegnato per valutare l'efficacia del trattamento della depressione sulle recidive di eventi coronarici o mortalità in pazienti con pregresso infarto miocardico, ha fallito nel dimostrare significativi benefici nei trattati con antidepressivi inibitori del re-uptake della serotonina rispetto ai trattati con usual care ${ }^{115}$ e che altri studi ${ }^{116}$ hanno dimostrato che gli effetti benefici sugli eventi cardiovascolari si manifestavano solo nel sottogruppo di pazienti che rispondevano bene alla terapia antidepressiva. Nella stessa direzione vanno i risultati di successivi studi ${ }^{117} \mathrm{di}$ più modeste dimensioni che tuttavia ribadiscono il concetto che se il trattamento della depressione è efficace

Tabella 1. Modalità di valutazione del rischio e di stratificazione prognostica.

La valutazione del rischio e la stratificazione prognostica nella prevenzione cardio-vascolare nei pazienti con cardiopatia ischemica manifesta deve essere effettuata in base ad una valutazione globale, in base ad un'attenta valutazione delle co-morbilità associate, anche quando sottese e non clinicamente manifeste

Nei pazienti affetti da cardiopatia ischemica sono particolarmente rilevanti, ai fini della valutazione del rischio CV, le abitudini di vita, le attitudini voluttuarie e l'attività fisica, il controllo del peso e della pressione arteriosa ed i farmaci assunti

Vanno considerate le comorbilità esistenti (diabete mellito manifesto, insuffcienza renale, equivalenti di rischio cardio-vascolare, comorbilià complesse associate (vedi Introduzione)

Vanno considerate le terapie effettuate e i rischi ad esse connessi (ipoglicemia, ipotensione, emorragia, deterioramento della funzione renale, ecc.)

Nel trattamento antitrombotico, in particolare, devono essere adeguatamente ponderati il rischio trombotico ed emorragico in ciascun singolo paziente

Nella valutazione del rischio e nella stratificazione prognostica dovrebbero essere ben individuati ed esplicitati, anche ai fini della continuità e del coordinamento delle cure, i pazienti a rischio CV elevato, meritevoli di un controllo preventivo dedicato

$\mathrm{CV}$, cardiovascolare. 
Tabella 2. Azioni da implementare in base alle caratteristiche e comorbidità del singolo paziente.

Oltre il 50\% della riduzione della mortalità per cardiopatia ischemica è imputabile alla riduzione dei fattori di rischio, mentre la restante porzione è imputabile a trattamenti farmacologici e non farmacologici

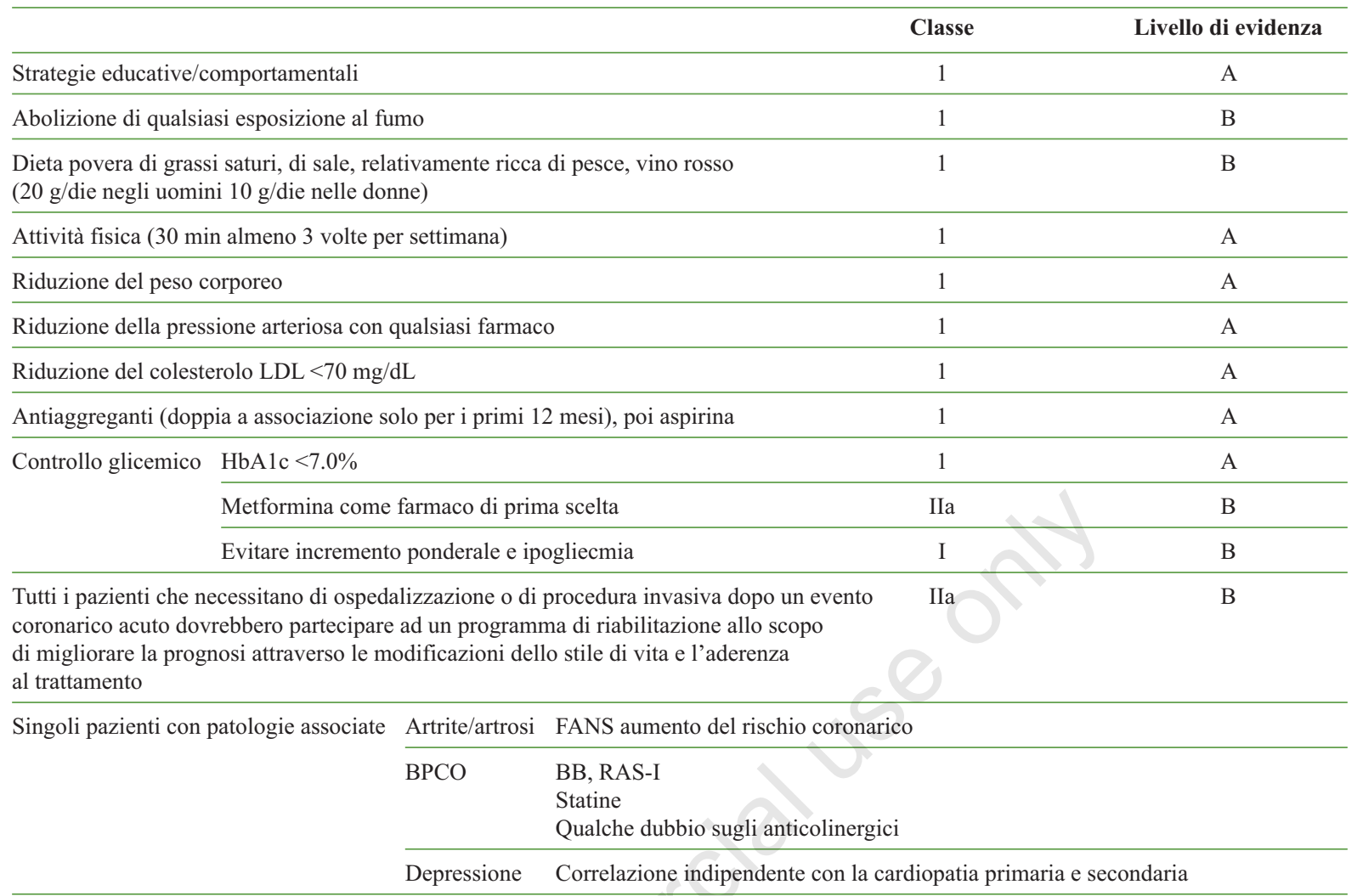

LDL, lipoproteine a bassa densità; FANS, farmaci anti-infiammatori non steroidei; BPCO, broncopneumopatia cronica ostruttiva; BB, $\beta$-bloccanti.

si possono ottenere benefici anche sul versante cardiovascolare.

\section{Conclusioni}

La proposta di un approccio omnicomprensivo (olistico) alla prevenzione cardiovascolare secondaria individualizzata sul singolo paziente è sintetizzata nelle Tabelle 1 (modalità di valutazione del rischio e di stratificazione prognostica) e 2 (azioni da implementare in base alle caratteristiche e comorbidità del singolo paziente).

\section{Bibliografia}

1. Perk J, De Backer G, Gohlke H, et al. European guidelines on cardiovascular disease prevention in clinical practice (version 2012). The Fifth Joint Task Force of the European Society of Cardiology and Other Societies on Cardiovascular Disease Prevention in Clinical Practice (constituted by representatives of nine societies and by invited experts). Eur Heart J 2012;33:1635-701.

2. Kotseva K, Wood D, De Backer G, et al. EUROASPIRE
III: a survey on the lifestyle, risk factors and use of cardioprotective drug therapies in coronary patients from 22 European countries. Eur J Cardiovasc Prev Rehabil 2009;16:121-37.

3. Kotseva K, Wood D, De Backer G, et al. EUROASPIRE III. Management of cardiovascular risk factors in asymptomatic high-risk patients in general practice: cross-sectional survey in 12 European countries. Eur J Cardiovasc Prev Rehabil 2010;17:530-40.

4. Dusseldorp E, van Elderen T, Maes S, et al. A metaanalysis of psychoeduational programs for coronary heart disease patients. Health Psychol 1999;18:506-19.

5. Rees K, Bennett P, West R, et al. Psychological interventions for coronary heart disease. Cochrane Database Syst Rev 2004;(2):CD002902.

6. Clark AM, Hartling L, Vandermeer B, McAlister FA. Meta-analysis: secondary prevention programs for patients with coronary artery disease. Ann Intern Med 2005; 143:659-72.

7. Auer R, Gaume J, Rodondi N, et al. Efficacy of in-hospital multidimensional interventions of secondary prevention after acute coronary syndrome: a systematic review and meta-analysis. Circulation 2008;117: 3109-17.

8. Linden W, Phillips MJ, Leclerc J. Psychological treatment of cardiac patients: a meta-analysis. Eur Heart J 2007;28:2972-84. 
9. Doll R, Peto R, Wheatley K, et al. Mortality in relation to smoking: 40 years' observations on male British doctors. BMJ 1994;309:901-11.

10. He J, Vupputuri S, Allen K, et al. Passive smoking and the risk of coronary heart disease - a meta-analysis of epidemiologic studies. N Engl J Med 1999;340:920-6.

11. Lancaster T, Stead L. Physician advice for smoking cessation. Cochrane Database Syst Rev 2004;(4):CD 000165 .

12. Stead LF, Perera R, Bullen C, et al. Nicotine replacement therapy for smoking cessation. Cochrane Database Syst Rev 2008;(1):CD000146.

13. Astrup A, Dyerberg J, Elwood P, et al. The role of reducing intakes of saturated fat in the prevention of cardiovascular disease: where does the evidence stand in 2010? Am J Clin Nutr 2011;93:684-8.

14. Corrao G, Bagnardi V, Zambon A, La Vecchia C. A meta-analysis of alcohol consumption and the risk of 15 diseases. Prev Med 2004;38:613-9.

15. Lollgen H, Bockenhoff A, Knapp G. Physical activity and all-cause mortality: an updated meta-analysis with different intensity categories. Int J Sports Med 2009;30:213-24.

16. Nocon M, Hiemann T, Muller-Riemenschneider F, et al. Association of physical activity with all-cause and cardiovascular mortality: a systematic review and metaanalysis. Eur J Cardiovasc Prev Rehabil 2008;15: 239-46.

17. Schnohr P, Scharling H, Jensen JS. Intensity versus duration of walking, impact on mortality: the Copenhagen City Heart Study. Eur J Cardiovasc Prev Rehabil 2007;14:72-8.

18. Piepoli MF, Davos C, Francis DP, Coats AJ. Exercise training meta-analysis of trials in patients with chronic heart failure (ExTraMATCH). BMJ 2004;328:189.

19. Taylor RS, Brown A, Ebrahim S, et al. Exercise-based rehabilitation for patients with coronary heart disease: systematic review and meta-analysis of randomized controlled trials. Am J Med 2004;116:682-92.

20. Berrington de Gonzalez A, Hartge P, Cerhan JR, et al. Body-mass index and mortality among 1.46 million white adults. N Engl J Med. 2010;363:2211-9.

21. Whitlock G, Lewington S, Sherliker P, et al. Body-mass index and cause-specific mortality in 900000 adults: collaborative analyses of 57 prospective studies. Lancet 2009;373:1083-96.

22. Zheng W, McLerran DF, Rolland B, et al. Association between body-mass index and risk of death in more than 1 million Asians. N Engl J Med. 2011;364:719-29.

23. Zanchetti A, Grassi G, Mancia G. When should antihypertensive drug treatment be initiated and to what levels should systolic blood pressure be lowered? A critical reappraisal. J Hypertens 2009;27:923-34.

24. Liu L, Zhang Y, Liu G, et al. The felodipine event reduction (FEVER) study: a randomized long-term placebo-controlled trial in Chinese hypertensive patients. J Hypertens 2005;23:2157-72.

25. Weber MA, Julius S, Kjeldsen SE, et al. Blood pressure dependent and independent effects of antihypertensive treatment on clinical events in the VALUE Trial. Lancet 2004;363:2049-51.

26. Neter JE, Stam BE, Kok FJ, et al. Influence of weight reduction on blood pressure: a meta-analysis of randomized controlled trials. Hypertension 2003;42:878-84.
27. Cornelissen VA, Fagard RH, Coeckelberghs E, Vanhees L. Impact of resistance training on blood pressure and other cardiovascular risk factors: a meta-analysis of randomized, controlled trials. Hypertension 2011;58:950-8.

28. McFadden CB, Brensinger CM, Berlin JA, Townsend RR. Systematic review of the effect of daily alcohol intake on blood pressure. Am J Hypertens 2005; 18:276-86.

29. He FJ, MacGregor GA. Salt reduction lowers cardiovascular risk: meta-analysis of outcome trials. Lancet 2011;378:380-2.

30. Mancia G, De Backer G, Dominiczak A, et al. 2007 Guidelines for the Management of Arterial Hypertension: The Task Force for the Management of Arterial Hypertension of the European Society of Hypertension (ESH) and of the European Society of Cardiology (ESC). J Hypertens 2007;25:1105-87.

31. Law MR, Morris JK, Wald NJ. Use of blood pressure lowering drugs in the prevention of cardiovascular disease: meta-analysis of 147 randomised trials in the context of expectations from prospective epidemiological studies. BMJ 2009;338:b1665.

32. Sever P. New hypertension guidelines from the National Institute for Health and Clinical Excellence and the British Hypertension Society. J Renin Angiotensin Aldosterone Syst 2006;7:61-3.

33. Piepoli MF, Corra U, Benzer W, et al. Secondary prevention through cardiac rehabilitation: from knowledge to implementation. A position paper from the Cardiac Rehabilitation Section of the European Association of Cardiovascular Prevention and Rehabilitation. Eur J Cardiovasc Prev Rehabil 2010;17:1-17.

34. Viberti G, Wheeldon NM. Microalbuminuria reduction with valsartan in patients with type 2 diabetes mellitus: a blood pressure-independent effect. Circulation 2002;106:672-8.

35. Turnbull F, Neal B, Algert C, et al. Effects of different blood pressure-lowering regimens on major cardiovascular events in individuals with and without diabetes mellitus: results of prospectively designed overviews of randomized trials. Arch Intern Med 2005;165:1410-9.

36. Baigent C, Blackwell L, Collins R, et al. Aspirin in the primary and secondary prevention of vascular disease: collaborative meta-analysis of individual participant data from randomised trials. Lancet 2009;373:1849-60.

37. Jardine MJ, Ninomiya T, Perkovic V, et al. Aspirin is beneficial in hypertensive patients with chronic kidney disease: a post-hoc subgroup analysis of a randomized controlled trial. J Am Coll Cardiol 2010;56:956-65.

38. de Lemos JA, Blazing MA, Wiviott SD, et al. Early intensive vs a delayed conservative simvastatin strategy in patients with acute coronary syndromes: phase $\mathrm{Z}$ of the A to Z trial. JAMA 2004;292:1307-16.

39. Ray KK, Cannon CP, McCabe CH, et al. Early and late benefits of high-dose atorvastatin in patients with acute coronary syndromes: results from the PROVE IT-TIMI 22 trial. J Am Coll Cardiol 2005;46:1405-10.

40. Schwartz GG, Olsson AG, Ezekowitz MD, et al. Atorvastatin for acute coronary syndromes. JAMA 2001; 286:533-5.

41. Amarenco P, Labreuche J. Lipid management in the prevention of stroke: review and updated meta-analysis of statins for stroke prevention. Lancet Neurol 2009; 8:453-63. 
42. Aung PP, Maxwell HG, Jepson RG, et al. Lipid-lowering for peripheral arterial disease of the lower limb. Cochrane Database Syst Rev 2007;(4):CD000123.

43. Sandhu S, Wiebe N, Fried LF, Tonelli M. Statins for improving renal outcomes: a meta-analysis. J Am Soc Nephrol. 2006;17:2006-16.

44. Gurbel PA, Bliden KP, Butler K, et al. Randomized double-blind assessment of the ONSET and OFFSET of the antiplatelet effects of ticagrelor versus clopidogrel in patients with stable coronary artery disease: the ONSET/OFFSET study. Circulation 2009;120:2577-85.

45. Wallentin L, Becker RC, Budaj A, et al. Ticagrelor versus clopidogrel in patients with acute coronary syndromes. N Engl J Med. 2009;361:1045-57.

46. Wiviott SD, Braunwald E, McCabe $\mathrm{CH}$, et al. Prasugrel versus clopidogrel in patients with acute coronary syndromes. N Engl J Med 2007;357:2001-15.

47. Chen ZM, Jiang LX, Chen YP, et al. Addition of clopidogrel to aspirin in 45,852 patients with acute myocardial infarction: randomised placebo-controlled trial. Lancet 2005;366:1607-21.

48. Sacco RL, Diener HC, Yusuf S, et al. Aspirin and extended-release dipyridamole versus clopidogrel for recurrent stroke. N Engl J Med. 2008;359:1238-51.

49. Diener HC, Bogousslavsky J, Brass LM, et al. Aspirin and clopidogrel compared with clopidogrel alone after recent ischaemic stroke or transient ischaemic attack in high-risk patients (MATCH): randomised, doubleblind, placebo-controlled trial. Lancet 2004;364: 331-7.

50. Mohr JP, Thompson JL, Lazar RM, et al. A comparison of warfarin and aspirin for the prevention of recurrent ischemic stroke. N Engl J Med. 2001;345:1444-51.

51. Liu M, Counsell C, Sandercock P. Anticoagulants for preventing recurrence following ischaemic stroke or transient ischaemic attack. Cochrane Database Syst Rev 2000;(2):CD000248.

52. Group U. Intensive blood-glucose control with sulphonylureas or insulin compared with conventional treatment and risk of complications in patients with type 2 diabetes (UKPDS 33). UK Prospective Diabetes Study (UKPDS) Group. Lancet 1998;352:837-53.

53. Patel A, MacMahon S, Chalmers J, et al. Intensive blood glucose control and vascular outcomes in patients with type 2 diabetes. N Engl J Med. 2008;358:2560-72.

54. [No authors listed]. Effect of intensive blood-glucose control with metformin on complications in overweight patients with type 2 diabetes (UKPDS 34). UK Prospective Diabetes Study (UKPDS) Group. Lancet 1998;352:854-65.

55. Duckworth W, Abraira C, Moritz T, et al. Glucose control and vascular complications in veterans with type 2 diabetes. N Engl J Med 2009;360:129-39.

56. Chow CK, Jolly S, Rao-Melacini P, et al. Association of diet, exercise, and smoking modification with risk of early cardiovascular events after acute coronary syndromes. Circulation 2010;121:750-8.

57. Bramlage P, Messer C, Bitterlich N, et al. The effect of optimal medical therapy on 1-year mortality after acute myocardial infarction. Heart 2010;96:604-9.

58. Critchley J, Capewell S. Smoking cessation for the secondary prevention of coronary heart disease. Cochrane Database Syst Rev 2004;(1):CD003041.
59. Rigotti NA, Munafo MR, Stead LF. Smoking cessation interventions for hospitalized smokers: a systematic review. Arch Intern Med 2008;168:1950-60.

60. He FJ, MacGregor GA. Effect of modest salt reduction on blood pressure: a meta-analysis of randomized trials. Implications for public health. J Hum Hypertens 2002;16:761-70.

61. Bibbins-Domingo K, Chertow GM, Coxson PG, et al. Projected effect of dietary salt reductions on future cardiovascular disease. N Engl J Med 2010;362:590-9.

62. Mozaffarian D, Rimm EB. Fish intake, contaminants, and human health: evaluating the risks and the benefits. JAMA 2006;296:1885-99.

63. Baigent C, Blackwell L, Emberson J, et al. Efficacy and safety of more intensive lowering of LDL cholesterol: a meta-analysis of data from 170,000 participants in 26 randomised trials. Lancet 2010;376:1670-81.

64. Heran BS, Chen JM, Ebrahim S, et al. Exercise-based cardiac rehabilitation for coronary heart disease. Cochrane Database Syst Rev 2011;(7):CD001800.

65. Franklin BA, Bonzheim K, Gordon S, Timmis GC. Safety of medically supervised outpatient cardiac rehabilitation exercise therapy: a 16-year follow-up. Chest 1998;114:902-6.

66. Romero-Corral A, Montori VM, Somers VK, et al. Association of bodyweight with total mortality and with cardiovascular events in coronary artery disease: a systematic review of cohort studies. Lancet 2006;368:666-78.

67. Hastie CE, Padmanabhan S, Slack R, et al. Obesity paradox in a cohort of 4880 consecutive patients undergoing percutaneous coronary intervention. Eur Heart J 2010;31:222-6.

68. Lavie CJ, Milani RV, Ventura HO. Obesity and cardiovascular disease: risk factor, paradox, and impact of weight loss. J Am Coll Cardiol 2009;53:1925-32.

69. Reboldi G, Gentile G, Angeli F, Verdecchia P. Blood pressure lowering in the oldest old. J Hypertens 2010;28:1373-6.

70. Messerli FH, Mancia G, Conti CR, et al. Dogma disputed: can aggressively lowering blood pressure in hypertensive patients with coronary artery disease be dangerous? Ann Intern Med 2006;144:884-93.

71. Verdecchia P, Reboldi G, Angeli F, et al. Systolic and diastolic blood pressure changes in relation with myocardial infarction and stroke in patients with coronary artery disease. Hypertension 2015;65:108-14.

72. Mancia G, Fagard R, Narkiewicz K, et al. 2013 ESH/ESC Guidelines for the management of arterial hypertension: The Task Force for the management of arterial hypertension of the European Society of Hypertension (ESH) and of the European Society of Cardiology (ESC). J Hypertens 2013;31:1281-357.

73. Gitt AK, Drexel H, Feely J, et al. Persistent lipid abnormalities in statin-treated patients and predictors of LDLcholesterol goal achievement in clinical practice in Europe and Canada. Eur J Prev Cardiol 2012;19:221-30.

74. Grigore L, Norata GD, Catapano AL. Combination therapy in cholesterol reduction: focus on ezetimibe and statins. Vasc Health Risk Manag 2008;4:267-78.

75. Robinson JG, Farnier M, Krempf M, et al. Efficacy and safety of alirocumab in reducing lipids and cardiovascular events. N Engl J Med 2015;372:1489-99.

76. Sabatine MS, Giugliano RP, Wiviott SD, et al. Efficacy 
and safety of evolocumab in reducing lipids and cardiovascular events. N Engl J Med 2015;372:1500-9.

77. Bhatt DL, Fox KA, Hacke W, et al. Clopidogrel and aspirin versus aspirin alone for the prevention of atherothrombotic events. N Engl J Med. 2006;354:1706-17.

78. CAPRIE Steering Committee. A randomised, blinded, trial of clopidogrel versus aspirin in patients at risk of ischaemic events (CAPRIE). CAPRIE Steering Committee. Lancet 1996;348:1329-39.

79. Borzì V, Fontanella A. The clinical impact of hypoglycemia in hospitalized patients. Ital J Med 2015;9:11-9.

80. Boyd CM, Leff B, Wolff JL, et al. Informing clinical practice guideline development and implementation: prevalence of coexisting conditions among adults with coronary heart disease. J Am Geriatr Soc 2011;59:797-805.

81. Haugen IK, Ramachandran VS, Misra D, et al. Hand osteoarthritis in relation to mortality and incidence of cardiovascular disease: data from the Framingham heart study. Ann Rheum Dis 2015;74:74-81.

82. Maradit-Kremers H, Crowson CS, Nicola PJ, et al. Increased unrecognized coronary heart disease and sudden deaths in rheumatoid arthritis: a population-based cohort study. Arthritis Rheum 2005;52:402-11.

83. Beinsberger J, Heemskerk JW, Cosemans JM. Chronic arthritis and cardiovascular disease: altered blood parameters give rise to a prothrombotic propensity. Semin Arthritis Rheum 2014;44:345-52.

84. Innala L, Moller B, Ljung L, et al. Cardiovascular events in early RA are a result of inflammatory burden and traditional risk factors: a five year prospective study. Arthritis Res Ther 2011;13:R131.

85. Kaplan MJ. Cardiovascular disease in rheumatoid arthritis. Curr Opin Rheumatol 2006;18:289-97.

86. Ikonomidis I, Lekakis JP, Nikolaou M, et al. Inhibition of interleukin- 1 by anakinra improves vascular and left ventricular function in patients with rheumatoid arthritis. Circulation 2008;117:2662-9.

87. Kobayashi H, Kobayashi Y, Giles JT, et al. Tocilizumab treatment increases left ventricular ejection fraction and decreases left ventricular mass index in patients with rheumatoid arthritis without cardiac symptoms: assessed using 3.0 tesla cardiac magnetic resonance imaging. J Rheumatol 2014;41:1916-21.

88. Bhala N, Emberson J, Merhi A, et al. Vascular and upper gastrointestinal effects of non-steroidal anti-inflammatory drugs: meta-analyses of individual participant data from randomised trials. Lancet 2013;382:769-79.

89. Grimaldi-Bensouda L, Rossignol M, Danchin N, et al. Risk of ST versus non-ST elevation myocardial infarction associated with non-steroidal anti-inflammatory drugs. Heart 2011;97:1834-40.

90. Schjerning Olsen AM, Fosbol EL, Lindhardsen J, et al. Duration of treatment with nonsteroidal anti-inflammatory drugs and impact on risk of death and recurrent myocardial infarction in patients with prior myocardial infarction: a nationwide cohort study. Circulation 2011;123:2226-35.

91. Olsen AM, Fosbol EL, Lindhardsen J, et al. Long-term cardiovascular risk of nonsteroidal anti-inflammatory drug use according to time passed after first-time myocardial infarction: a nationwide cohort study. Circulation 2012;126:1955-63.

92. Agarwal S, Rokadia H, Senn T, Menon V. Burden of cardiovascular disease in chronic obstructive pulmonary disease. Am J Prev Med 2014;47:105-14.

93. Miller J, Edwards LD, Agusti A, et al. Comorbidity, systemic inflammation and outcomes in the ECLIPSE cohort. Respir Med 2013;107:1376-84.

94. Bursi F, Vassallo R, Weston SA, et al. Chronic obstructive pulmonary disease after myocardial infarction in the community. Am Heart J 2010;160:95-101.

95. Camilli AE, Robbins DR, Lebowitz MD. Death certificate reporting of confirmed airways obstructive disease. Am J Epidemiol 1991;133:795-800.

96. Puente-Maestu L, Calle M, Ortega-Gonzalez A, et al. Multicentric study on the $\beta$-blocker use and relation with exacerbations in COPD. Respir Med 2014;108: 737-44.

97. Quint JK, Herrett E, Bhaskaran K, et al. Effect of $\beta$ blockers on mortality after myocardial infarction in adults with COPD: population based cohort study of UK electronic healthcare records. BMJ 2013;347: f6650.

98. Zeng LH, Hu YX, Liu L, et al. Impact of $\beta 2$-agonists, $\beta$-blockers, and their combination on cardiac function in elderly male patients with chronic obstructive pulmonary disease. Clin Interv Aging 2013;8:1157-65.

99. Mancini GB, Etminan M, Zhang B, et al. Reduction of morbidity and mortality by statins, angiotensin-converting enzyme inhibitors, and angiotensin receptor blockers in patients with chronic obstructive pulmonary disease. J Am Coll Cardiol 2006;47:2554-60.

100. Petersen H, Sood A, Meek PM, et al. Rapid lung function decline in smokers is a risk factor for COPD and is attenuated by angiotensin-converting enzyme inhibitor use. Chest 2014;145:695-703.

101. Horita N, Miyazawa N, Kojima R, et al. Statins reduce all-cause mortality in chronic obstructive pulmonary disease: a systematic review and meta-analysis of observational studies. Respir Res 2014;15:80.

102. Janda S, Park K, FitzGerald JM, et al. Statins in COPD: a systematic review. Chest 2009;136:734-43.

103. Criner GJ, Connett JE, Aaron SD, et al. Simvastatin for the prevention of exacerbations in moderate-to-severe COPD. N Engl J Med 2014;370:2201-10.

104. Wilchesky M, Ernst P, Brophy JM, et al. Bronchodilator use and the risk of arrhythmia in COPD: part 1: Saskatchewan cohort study. Chest 2012;142:298-304.

105. Singh S, Loke YK, Furberg CD. Inhaled anticholinergics and risk of major adverse cardiovascular events in patients with chronic obstructive pulmonary disease: a systematic review and meta-analysis. JAMA 2008;300: 1439-50.

106. Celli B, Decramer M, Kesten S, et al. Mortality in the 4-year trial of tiotropium (UPLIFT) in patients with chronic obstructive pulmonary disease. Am J Respir Crit Care Med 2009;180:948-55.

107. Carney RM, Freedland KE. Depression in patients with coronary heart disease. Am J Med 2008;121:S20-7.

108. Lichtman JH, Froelicher ES, Blumenthal JA, et al. Depression as a risk factor for poor prognosis among patients with acute coronary syndrome: systematic review and recommendations: a scientific statement from the American Heart Association. Circulation 2014;129: 1350-69.

109. Rosengren A, Hawken S, Ounpuu S, et al. Association of psychosocial risk factors with risk of acute myocar- 
dial infarction in 11119 cases and 13648 controls from 52 countries (the INTERHEART study): case-control study. Lancet 2004;364:953-62.

110. Engum A. The role of depression and anxiety in onset of diabetes in a large population-based study. J Psychosom Res 2007;62:31-8.

111. Davidson K, Jonas BS, Dixon KE, Markovitz JH. Do depression symptoms predict early hypertension incidence in young adults in the CARDIA study? Coronary Artery Risk Development in Young Adults. Arch Intern Med 2000;160:1495-500.

112. Louise S, Warrington NM, McCaskie PA, et al. Associations between anxious-depressed symptoms and cardiovascular risk factors in a longitudinal childhood study. Prev Med 2012;54:345-50.

113. Musselman DL, Evans DL, Nemeroff CB. The relationship of depression to cardiovascular disease: epidemiology, biology, and treatment. Arch Gen Psychiatry 1998;55:580-92.
114. Thombs BD, de Jonge P, Coyne JC, et al. Depression screening and patient outcomes in cardiovascular care: a systematic review. JAMA 2008;300:2161-71.

115. Berkman LF, Blumenthal J, Burg M, et al. Effects of treating depression and low perceived social support on clinical events after myocardial infarction: the Enhancing Recovery in Coronary Heart Disease Patients (ENRICHD) Randomized Trial. JAMA 2003; 289:3106-16.

116. de Jonge P, Honig A, van Melle JP, et al. Nonresponse to treatment for depression following myocardial infarction: association with subsequent cardiac events. Am J Psychiatry 2007;164:1371-8.

117. Davidson KW, Rieckmann N, Clemow L, et al. Enhanced depression care for patients with acute coronary syndrome and persistent depressive symptoms: coronary psychosocial evaluation studies randomized controlled trial. Arch Intern Med 2010;170:600-8. 


\title{
La prevenzione cardiovascolare nello scompenso cardiaco
}

\author{
Rodolfo Cavaliere, ${ }^{1}$ Fernando Gallucci, ${ }^{2}$ Giovanni Mathieu ${ }^{3}$ \\ ${ }^{1}$ Medicina Interna, FADOI Piemonte; ${ }^{2}$ UOC di Medicina Interna 3, AORN A. Cardarelli, Napoli; ${ }^{3}$ Medicina Interna, FADOI \\ Piemonte, Italia
}

\section{Definizione}

Lo scompenso cardiaco (SC) è una sindrome clinica complessa causata da un'anomalia strutturale e/o funzionale in grado di alterare il riempimento ventricolare e/o la fase di eiezione con conseguente perdita della capacità del cuore a pompare quantità di sangue adeguate alle richieste metaboliche dei tessuti se non attraverso un aumento della pressione telediastolica del ventricolo sinistro. ${ }^{1}$ Lo SC origina quindi da una compromissione della funzionalità miocardica con una possibile presenza contemporanea di disfunzione sistolica e diastolica del ventricolo sinistro. ${ }^{2,3}$

\section{Classificazione}

L'importanza attribuita alla frazione d'eiezione (FE) nella classificazione delle forme di SC è legata alla sua relazione con alcune caratteristiche del paziente (comorbidità, fattori prognostici e risposta alle diverse terapie $)^{4}$ ed al fatto che i pazienti arruolati nella maggior parte dei trials sono selezionati sulla base di questo parametro.

Si distingue pertanto: i) scompenso cardiaco con frazione di eiezione ridotta ( $\mathrm{HFrEF}$ o SC cosiddetto sistolico) con $\mathrm{FE} \leq 40 \%$; ii) scompenso cardiaco con frazione di eiezione conservata (HF $p$ EF o SC cosiddetto diastolico) con $\mathrm{FE} \geq 50$; iii) scompenso cardiaco con frazione di eiezione borderline (HF $p$ EF borderline) con FE compresa tra 41 e $49 \%$.

Corrispondente: Giovanni Mathieu, Past President nazionale FADOI, FADOI Piemonte, Corso Porporato 25, 10064 Pinerolo (TO), Italia.

E-mail: nanni.mathieu@tiscali.it

Articolo pubblicato secondo la Creative Commons Attribution NonCommercial 3.0 License (CC BY-NC 3.0).

CCopyright R. Cavaliere et al., 2015

Licensee PAGEPress, Italy

QUADERNI - Italian Journal of Medicine 2015; 3:339-355

\section{Stadi dello scompenso cardiaco}

Sia gli stadi dello SC secondo l'American College of Cardiology Foundation/American Heart Association (ACCF/AHA) ${ }^{3}$ che la classificazione funzionale della New York Heart Association (NYHA) ${ }^{5}$ forniscono informazioni utili circa la presenza e la severità della patologia.

I primi pongono l'attenzione sull'evoluzione anatomo-funzionale della malattia, tenendo conto dei fattori di rischio e delle anomalie della struttura cardiaca mentre le classi NYHA fanno riferimento allo stato sintomatico. Interventi terapeutici nei vari stadi dello $\mathrm{SC}$ tendono a modificare i fattori di rischio (stadio A), a trattare la patologia strutturale cardiaca (stadio B) ed a ridurre la morbidità e la mortalità (stadio $\mathrm{C}$ e D).

\section{Classificazione in stadi}

Stadio A: pazienti ad alto rischio di sviluppare uno SC ma senza danni strutturali del cuore.

Stadio B: pazienti con alterazioni strutturali del cuore in assenza di sintomi di SC.

Stadio C: pazienti che hanno avuto o hanno sintomi di SC associati ad una sottostante alterazione strutturale cardiaca.

Stadio D: pazienti con alterazioni strutturali avanzate e grave $\mathrm{SC}$, con sintomi a riposo, che richiedono trattamenti specifici come supporti circolatori meccanici, infusione continua di farmaci inotropi, trapianto cardiaco, ospedalizzazione.

Seppure con riproducibilità e validità discutibili, ${ }^{6}$ la classificazione funzionale NYHA rappresenta un predittore indipendente di mortalità. ${ }^{7}$

\section{Classificazione funzionale (NYHA)}

Classe I: nessuna limitazione nelle normali attività quotidiane; l'esercizio fisico ordinario non causa affaticamento, dispnea o cardiopalmo (disfunzione ventricolare asintomatica).

Classe II: limitazione delle attività ordinarie; l'attività fisica ordinaria determina affaticamento, palpitazioni o dispnea (disfunzione media).

Classe III: marcata limitazione delle attività ordinarie che determinano sintomi, con scomparsa degli stessi a riposo (disfunzione moderata). 
Classe IV: sintomi di SC presenti anche a riposo (disfunzione severa).

\section{Epidemiologia}

La prevalenza dello SC nella popolazione generale è compresa tra 2 e $3 \%$, con marcato incremento dopo i 75 anni (percentuali comprese tra il 10 ed il 20\%); dopo i 60 anni il $10 \%$ dei soggetti di sesso maschile ed il 6\% di sesso femminile presenta un quadro di SC. ${ }^{8}$ Nei pazienti più giovani lo $\mathrm{SC}$ è più frequente nel genere maschile, mentre nell'anziano la prevalenza è simile in entrambi i sessi. Nel sesso femminile predominano le forme di SC con funzione sistolica preservata $^{9-14}$ (eziologia prevalente: cardiopatia ipertensiva o cardiopatia diabetica).

Nei Paesi europei lo SC rappresenta il 5\% delle cause di ricovero ospedaliero; si stima che circa il 10\% dei posti-letto siano occupati da pazienti con questa patologia. La durata della degenza media è molto variabile ${ }^{15}$ a seconda delle realtà geografiche $(4$ giorni di degenza media negli Stati Uniti a fronte di 8 giorni nei Paesi europei), con tasso di mortalità intraospedaliera tra 3 e $9 \%$. Nel follow-up a 2-3 mesi il tasso di mortalità risulta elevato (9-13\%), con altrettanto elevato rischio di riospedalizzazione (24-30\%).

Lo SC è responsabile di elevati costi sanitari (circa il $2 \%$ della spesa sanitaria $)^{16,17}$ per via dei ricoveri ospedalieri, degli accessi al Pronto Soccorso (PS) e delle ripetute visite mediche.

La sopravvivenza dopo la diagnosi è migliorata nelle ultime decadi (riduzione del tasso di mortalità età-correlato e incremento dell'età media al momento del decesso). ${ }^{18-22}$ Nonostante i progressi nella terapia, la prognosi rimane a tutt'oggi sfavorevole, con mortalità a 5 anni dalla diagnosi assai elevata (pari a circa il 50\%), simile a quella di molte patologie oncologiche. ${ }^{23-25}$

Il profilo emodinamico dei pazienti con $\mathrm{SC}$ si è modificato nelle più recenti decadi. ${ }^{26}$ Tra i pazienti ricoverati per SC, il $40 \%$ ha una funzione sistolica conservata ( $\mathrm{FE}>50 \%$ ), specie nei soggetti anziani, di sesso femminile, obesi, ipertesi ed in fibrillazione atriale; questi pazienti hanno un tasso di mortalità solo lievemente più basso rispetto ai pazienti con funzione sistolica ridotta; inoltre le cause di morte nei pazienti con frazione d'eiezione conservata (HF $p \mathrm{EF}$ ) spesso non sono attribuibili a cause cardiovascolari, quanto piuttosto alle comorbilità presenti. ${ }^{27,28}$

Lo studio TEMISTOCLE, ${ }^{29}$ survey prospettica realizzata nel 2000, ha raccolto i dati relativi a 2127 pazienti del mondo reale ricoverati per SC in 167 reparti cardiologici ed in 250 reparti di Medicina Interna italiani. Il fenotipo dei pazienti con SC che afferivano ai reparti internistici è risultato ben diffe- rente rispetto a quello dei pazienti ricoverati nei reparti cardiologici: pazienti più anziani (età media 76 anni; $3 / 4$ over 70 anni); eziologia prevalentemente riconducibile ad una cardiopatia ischemica e/o ipertensiva; maggior prevalenza di soggetti di genere femminile; presenza di comorbilità nel $70-80 \%$ dei casi (elevate percentuali di fibrillazione atriale, insufficienza renale, diabete mellito, broncopneumopatia cronica ostruttiva [BPCO], anemia); $40 \%$ dei casi con funzione sistolica conservata ( $\mathrm{FE}>40 \%)$.

Nei pazienti osservati in entrambi i setting di ricovero, veniva confermata una mortalità intra-ricovero del 5 e del $15 \%$ dopo 6 mesi dalla dimissione, una riospedalizzazione a 6 mesi nel $45 \%$ dei casi, e solo poco più della metà di questi pazienti veniva inserito in un programma di controllo a distanza.

Nel corso del 2006-2007, la Federazione delle Associazioni dei Dirigenti Ospedalieri Internisti (FADOI) ha realizzato un secondo studio epidemiologico in area internistica, denominato CONFINE, ${ }^{30}$ arruolando 1411 pazienti ricoverati per SC in 91 reparti di Medicina Interna. L'età media dei pazienti ricoverati è risultata pari a 78,7 anni, con una percentuale di ultra settantenni dell' $83,5 \%$. I pazienti arruolati nello studio presentavano le seguenti caratteristiche: classe NYHA III-IV nell' $81,7 \%$ dei casi; cardiopatia ischemica e/o ipertensiva quale eziologia predominante; sovrappeso o obesità nel $62 \%$ della casistica; solo il $10 \%$ con SC isolato; comorbilità più frequenti riconducibili ad ipertensione arteriosa, insufficienza renale, anemia, diabete mellito, BPCO, demenza; fibrillazione atriale nel $42,7 \%$ dei casi; indice di Barthel medio pari a 56; alta percentuale di soggetti con deficit funzionale e con impatto negativo sulla qualità di vita (valutata con il Minnesota living with heart failure questionnaire).

In una sotto-analisi dello studio CONFINE ${ }^{31}$ è emerso che il $23,1 \%$ dei pazienti arruolati era di età superiore a 85 anni e l' $8,8 \%$ a 90 anni. Nel grande anziano prevalevano quali comorbilità insufficienza renale, anemia, fibrillazione atriale; era inoltre più elevata la presenza di cachessia $(13,4 \%)$, disabilità e demenza, con conseguente elevata mortalità e peggioramento dello SC durante il ricovero.

\section{Stratificazione prognostica}

La stratificazione prognostica di un paziente con SC è particolarmente complessa per l'associazione di altre patologie che spesso interferiscono negativamente con la prognosi. Un primo dato prognostico è collegato all'etiopatogenesi della cardiopatia con valori prognostici più negativi per la cardiomiopatia da HIV con mortalità dell' $80 \%$ a 5 anni, più favorevole fra le etiopatogenesi, con evoluzione prevalente verso la guarigione, è quella post partum. A livello intermedio si pongono le forme 
di cardiopatia ischemica e dilatativa. ${ }^{32}$ L'età è un fattore aggravante la prognosi con mortalità a 5 anni che raddoppia dall'età tra i 50 ed i 70 anni (33\% di mortalità) agli ultrasettantenni (50\%), ${ }^{33}$ mentre negli ultraottantenni è ulteriormente peggiorata. ${ }^{34}$

La prognosi è collegata alla classe funzionale NYHA, quindi con bassa mortalità in classe NYHA I (5-19\% a 5 anni) che diventa più elevata in classe NYHA IV (44-64\% a 6-12 mesi); la prognosi è intermedia nelle classi NYHA II e III (15-40\% a 4 anni). ${ }^{7}$

I valori di pressione arteriosa sistolica (PAS) sembrano influenzare la prognosi, con un tasso di mortalità che diminuisce nei ricoverati da valori pari al 7,2\% nei pazienti con PAS $<120 \mathrm{mmHg}$ ad una mortalità dell' $1,7 \%$ nei pazienti con PAS $>160 \mathrm{mmHg}{ }^{35}$

Molti dati di laboratorio sono stati usati per una valutazione prognostica; un peggioramento della prognosi viene correlato a valori di BNP e di troponina elevati ${ }^{36,37} \mathrm{e}$ con bassi valori di sodio (ESCAPE trial). ${ }^{38}$ La presenza di un allargamento del QRS all'elettrocardiogramma identifica una prognosi che peggiora tanto più si allarga la durata del QRS, con mortalità ad un anno del $60 \%$ con un QRS $>220 \mathrm{~ms}^{39}{ }^{39}$ Anche la presenza di fibrillazione atriale ha un indice prognostico negativo raddoppiando il rischio di mortalità. ${ }^{40}$ La presenza di comorbilità aggrava la prognosi nei pazienti anziani: quella che peggiora maggiormente la prognosi è l'insufficienza renale cronica (IRC). Se è presente una IRC il rischio relativo di mortalità è pari a 2,85 per un filtrato glomerulare (GFR) $<44 \mathrm{~mL} / \mathrm{min}$ se confrontato con un GFR $>76 \mathrm{~mL} / \mathrm{min}^{.}{ }^{41-43} \mathrm{Nel}$ DigTrial la mortalità in presenza di SC è risultato inversamente proporzionale al GFR calcolato. La presenza di albuminuria è stata identificata come un altro forte marcatore prognostico sfavorevole. In un sottostudio dello studio CHARM (candesartan in heart failure: assessment of reduction in mortality and morbidity), su 2310 pazienti con SC, è stato misurato il rapporto albumina-creatinina di base e durante il follow-up. Il $30 \%$ aveva microalbuminuria e $1 ' 11 \%$ macroalbuminuria, sia con funzione ventricolare conservata che depressa. La presenza di qualsiasi tipo di albuminuria aumentava il rischio di eventi cardiaci avversi. ${ }^{44}$

Altra patologia associata a un peggioramento prognostico è la BPCO sia per l'aumento del rischio di ospedalizzazione $(25,7 v s 21,6 \%)$ che per la mortalità nei ricoverati $\left(35,4\right.$ vs 29,1\%), ${ }^{45,46}$ dato risultato ancora più rilevante nello studio di Holguin ${ }^{47}$ ed in quello del Northern California Kaiser permanent medical care program (KPNC) con rischio relativo di mortalità nei pazienti con SC associato a BPCO pari al $3,53 .{ }^{48} \mathrm{La}$ presenza di sleep apnea peggiora la prognosi e aumenta la mortalità. ${ }^{49}$

Frequente è una anemia associata allo SC; molti studi considerano come anemia valori di emoglobina $\mathrm{Hb})$ differenti (la maggior parte considera un valore di cut-off di $\mathrm{Hb}<12 \mathrm{~g} / \mathrm{dL}$ ) ma sembra certo che l'anemia sia un fattore di rischio indipendente di mortalità e morbilità in questi pazienti. ${ }^{50} \mathrm{Nel}$ CHARM program e nel Renaissance study $y^{51}$ la mortalità passa dal $28 \%$ ad un anno nei soggetti anemici al $16 \%$ dei non anemici. L'incremento del rischio di mortalità nei pazienti anemici passa dal 16 al 38\% per ogni grammo di $\mathrm{Hb}$ al di sotto dei $12 \mathrm{~g} / \mathrm{dL} .{ }^{52}$

Il diabete peggiora la prognosi nei pazienti con SC, maggiormente nei soggetti con bassa FE. ${ }^{53,54}$ Una prognosi negativa sembra associata all'aumento dell'HbA1c ${ }^{55}$ (CIBIS II, BEST, COPERNICUS, MERIT-HF).

La depressione o un trattamento antidepressivo in atto sembrano peggiorare la prognosi dello SC con un rischio univocamente accertato, anche elevato, in numerosi studi, seppure di piccole dimensioni, dell'ordine di oltre il 50\%. ${ }^{56,57}$ Altra condizione clinica che peggiora in modo significativo la prognosi dello SC è rappresentata dalla presenza di cachessia cardiaca. ${ }^{58,59}$

Una sintesi dei fattori prognostici negativi nello $\mathrm{SC}$ è riportata in un lavoro di Georghiade ${ }^{60}$ che riguarda i pazienti con $\mathrm{SC}$ acuto.

Da questa somma di condizioni cliniche associate ad una prognosi peggiorativa sono stati elaborati numerosi score di valutazione prognostica dello SC cronico e del rischio di ospedalizzazione. Per quanto concerne il quadro di $\mathrm{SC}$ acuto lo score più semplice, rapido e rispondente alla clinica è lo schema del gruppo di Stevenson ${ }^{61}$ fondato sui segni e sintomi indicativi di congestione o bassa portata, valutabili al letto del malato.

\section{Obiettivi terapeutici nel paziente con scompenso cardiaco}

Gli obiettivi terapeutici nei pazienti con SC non differiscono in modo sostanziale da quelli di ogni altra condizione clinica cardiovascolare. ${ }^{62}$ Essi sono riassumibili nei seguenti punti: i) riduzione della mortalità (live longer): circa la metà dei pazienti con $\mathrm{SC}$ muore di morte improvvisa perlopiù per aritmia maligna e la restante metà per progressione verso una forma di $\mathrm{SC}$ refrattario; ii) riduzione della morbilità (feel better): riduzione di segni e sintomi; aumento della capacità di esercizio fisico; miglioramento della qualità di vita; riduzione delle ospedalizzazioni; impostazione di cure palliative laddove indicate; iii) prevenzione del peggioramento verso uno $\mathrm{SC}$ avanzato o terminale, attraverso la prevenzione della progressione del danno cardiaco e la riduzione del rimodellamento maladattativo del ventricolo sinistro.

Il percorso clinico-assistenziale per realizzare una prevenzione cardiovascolare a tutto campo nei pazienti con SC prevede le seguenti azioni: identificazione dei pazienti ad elevato rischio di sviluppare SC; 
identificazione dei pazienti portatori di cardiopatia strutturale ancora asintomatica, passibile di evoluzione peggiorativa; accesso in modo equo alle cure preventive dimostrate efficaci; applicazione delle migliori pratiche cliniche basate sulle evidenze.

Sono elementi cardine del management dello SC: educazione sanitaria dei pazienti e dei loro familiari, adozione di un corretto stile di vita; utilizzo dei farmaci supportati dalle evidenze (per meccanismo di azione e dosaggio) seguendo le raccomandazioni delle linee guida; aderenza alla terapia ed organizzazione delle cure mediche nel lungo periodo (continuità assistenziale tra i differenti setting di cura; follow-up; supporto clinico, socioeconomico, psicologico).

\section{Interventi non farmacologici}

I pazienti con $\mathrm{SC}$ dovrebbero ricevere un programma educazionale specifico ${ }^{2,3,63-68}$ che permetta di riconoscere e monitorare i sintomi della patologia, con incentivo a seguire le prescrizioni non farmacologiche (dieta, attività fisica, aderenza ai farmaci prescritti, monitoraggio quotidiano del peso corporeo). Tale programma educazionale migliora la conoscenza della patologia, l'autocontrollo, l'aderenza alla terapia farmacologica e riduce l'intervallo di tempo per una ri-ospedalizzazione e la durata della degenza ospedaliera. ${ }^{2}$ Risultati vantaggiosi si ottengono sia se il programma viene attuato al momento della dimissione (ridotta durata della degenza, minori costi, ridotta mortalità a 6 mesi) ${ }^{65}$ che a domicilio col coinvolgimento anche dei familiari (ridotta frequenza di accessi al Pronto Soccorso e di ricoveri non programmati). ${ }^{67-69}$

Numerose revisioni sistematiche, compresa una updated Cochrane review, ${ }^{70}$ e meta-analisi di piccoli studi hanno dimostrato che il training fisico migliora la tolleranza allo sforzo e la qualità di vita, riducendo il tasso di ospedalizzazione nei pazienti con SC. . $^{71-75}$

Anche il supporto sociale è rilevante per migliorare l'aderenza al trattamento e promuovere un sano stile di vita, ${ }^{76}$ essendo dimostrato che la perdita di rapporti sociali si associa a tassi più elevati di ospedalizzazione $^{77,78} \mathrm{e}$ ad un aumentato rischio di morte. ${ }^{79,80}$

Dati osservazionali provenienti da studi minori suggeriscono un'associazione tra l'entità dell 'apporto di sodio con la dieta ed il rischio di ospedalizzazione, ${ }^{81,82}$ in contrasto con altre esperienze che hanno segnalato un peggioramento del profilo neuro-ormonale in corso di restrizione di sodio. ${ }^{83-85} \mathrm{E}$ anche dimostrato che l'aderenza a questo regime dietetico è piuttosto scarsa. ${ }^{86}$ Le più recenti linee guida sullo SC considerano ragionevole un regime dietetico che preveda un apporto di $1,5 \mathrm{~g} /$ die di sodio per la maggior parte dei pazienti con $\mathrm{SC}$ in stadio A e B; $;^{87-92}$ per i pazienti appartenenti agli stadi C e D, pur non essendovi al momento dati suffi- cienti in letteratura, andrebbe considerato un apporto di sodio $<3 \mathrm{~g} /$ die per migliorare la sintomatologia. ${ }^{3}$

Allo stesso scopo, nei pazienti con SC grave va incoraggiata anche la riduzione dell 'introito giornaliero di liquidi (max 1,5-2 L/die) ${ }^{93}$ La restrizione idrica correlata al peso corporeo $(30 \mathrm{~mL} / \mathrm{kg}$ di peso corporeo ovvero $35 \mathrm{~mL} / \mathrm{kg}$ se il peso corporeo supera gli $85 \mathrm{~kg}$ ) può ridurre la sete.

La malnutrizione va prevenuta o corretta, con una sana ed adeguata alimentazione. I pazienti con SC che hanno un indice di masa corporea (BMI) tra 30 e 35 $\mathrm{kg} / \mathrm{m}^{2}$ hanno una prognosi migliore con minore mortalità e minori tassi di ospedalizzazione rispetto ai pazienti con BMI nei limiti della norma. ${ }^{94}$ Peraltro, i pazienti marcatamente obesi possono avere una prognosi peggiore rispetto a quelli normopeso, con una distribuzione ad U dei tassi di mortalità correlati al peso corporeo. ${ }^{95}$

Ad eccezione dei pazienti con cardiomiopatia alcolica o altre controindicazioni, nei pazienti con SC le indicazioni generali consigliano due bicchieri di vino al giorno nei maschi ed un bicchiere di vino al giorno nelle donne.

Il fumo di sigaretta e/o l'uso di droghe vanno, ovviamente, aboliti.

Il paziente con SC va incentivato ad uscire di casa ed organizzare il proprio tempo libero, secondo le proprie capacità fisiche.

I pazienti stabili possono avere una normale attività sessuale che non provochi sintomi indesiderati.

I pazienti con SC devono essere sottoposti annualmente a vaccinazione antinfluenzale ed antipneumococcica.

Va posta attenzione ai sintomi della depressione e/o di un deficit cognitivo, condizioni non infrequenti nei pazienti con SC, che vanno trattate prontamente ed adeguatamente.

I disturbi del sonno, frequenti nei pazienti con $\mathrm{SC},{ }^{96}$ vanno sistematicamente ricercati. Il trattamento con la ventilazione meccanica a pressione positiva (CPAP) notturna ha dimostrato di ridurre l'indice apnea-ipopnea, migliorare l'ossigenazione notturna, incrementare la FE, ridurre la attivazione adrenergica, incrementare l'autonomia di marcia. ${ }^{97}$ Studi minori hanno suggerito che la CPAP può migliorare la funzione cardiaca e la qualità di vita (HRQOL) nei pazienti con SC ed associata sindrome da apnee ostruttive nel sonno (OSAS) ${ }^{98,99}$ Andranno attuate anche le misure comportamentali preventive dell'OSAS. ${ }^{2}$

Le linee guida internazionali suggeriscono di evitare i farmaci anti-infiammatori non steroidei (FANS) nei pazienti affetti da SC cronico per l'aumento del rischio di ritenzione idro-salina; vi è, inoltre, un aumento delle prime ospedalizzazioni per SC nella popolazione generale. ${ }^{100}$ Secondo le raccomandazioni dell'AHA andrebbe evitata la somministrazione dei COXIB nei pazienti ad alto rischio cardiovascolare o 
con malattia cardiovascolare documentata e consigliato l'uso di analgesici alternativi. È stato dimostrato anche un incremento di mortalità e morbilità cardiovascolare dose-dipendente con l'uso di COXIB o FANS non selettivi per cui andrebbe evitata la loro somministrazione a qualunque dosaggio (con l'eccezione di ibuprofene e naprossene da non assumere a dosi elevate). ${ }^{101}$ Inoltre, vi sono evidenze circa l'associazione dell'uso di FANS con un aumentato rischio di comparsa di fibrillazione atriale. ${ }^{102}$

Anche l'uso dell'eritropoietina andrebbe evitato nei pazienti con SC, per l'aumento del rischio di mortalità e per l'incremento di eventi cardiovascolari. ${ }^{103}$

\section{Interventi farmacologici}

Gli interventi farmacologici nel paziente con SC sono volti a migliorare la sintomatologia, a prevenire l'ospedalizzazione ed a ridurre la mortalità. ${ }^{2}$ Questi ultimi due obiettivi sono indicatori della efficacia nel rallentare o prevenire il progressivo peggioramento della malattia. Sebbene l'attenzione dei trials sia focalizzata sulla mortalità, la prevenzione dell'ospedalizzazione viene attualmente riconosciuta quale obiettivo importante sia per i pazienti che per il Servizio Sanitario Nazionale. ${ }^{104}$

Per quanto riguarda la frazione d'eiezione ridotta (HFrEF), tre classi di farmaci antagonisti neuro-umorali sono di importanza fondamentale nel modificarne il decorso e la prognosi (con dosi basse all'inizio della terapia, da incrementare con gradualità fino al raggiungimento delle dosi target): gli ACE inibitori (ACE-I) o gli antagonisti recettoriali dell'angiotensina (ARBs), i $\beta$-bloccanti (BB) e gli anti aldosteronici (MRA), comunemente associati ad un diuretico per alleviare i segni ed i sintomi della congestione. ${ }^{2}$

È raccomandato l'uso di un ACE-I in aggiunta ad un BB, o di un BB ad un ACE-I (o ARB se l'ACE-I non è ben tollerato) nei pazienti con $\mathrm{FE} \leq 40 \%$ per ridurre il rischio di ospedalizzazioni per SC e di morte prematura. ${ }^{105-}$ ${ }^{116}$ L'aggiunta di un MRA al trattamento con ACE-I (o ARB se l'ACE-I non è ben tollerato) e BB è raccomandata allo stesso scopo in quelli con $\mathrm{FE} \leq 35 \%{ }^{117,118}$

I benefici della terapia a lungo termine con BB (bisoprololo, metoprololo a rilascio controllato e carvedilolo) sulla sintomatologia, sul miglioramento delle condizioni cliniche generali, sulla sopravvivenza e sul rischio combinato di morte o ospedalizzazione sono stati ampiamente dimostrati. ${ }^{119-128}$ Essi vanno prescritti precocemente a tutti i pazienti con $\mathrm{HF} r \mathrm{EF}$ stabile in assenza di controindicazioni o di intolleranza, ${ }^{126} \mathrm{con}$ particolare attenzione alla presenza di alcune comorbidità, molto frequenti nei pazienti complessi, come ad esempio la BPCO o le bradiaritmie; comunque, con l'avvento di BB $\beta 1$ selettivi se ne è reso possibile, con le opportune cautele, l'uso anche in pazienti con
BPCO severa. Il trattamento va iniziato con dosi molto basse da incrementare gradualmente, prestando attenzione, in particolare, a quattro eventi avversi possibili: ritenzione idrica con peggioramento dello SC, astenia profonda, bradicardia, ipotensione arteriosa. ${ }^{3}$

Gli effetti benefici dell'ace-inibizione sono stati osservati nei pazienti con e senza cardiopatia ischemica, in tutte le classi NYHA, con riduzione del rischio di ospedalizzazione per SC e di morte, anche in presenza di disfunzione sistolica del ventricolo sinistro asintomatica ed in pazienti più anziani con SC ed insufficienza renale cronica. ${ }^{129}$ Per i possibili effetti collaterali, dovrebbero essere prescritti con cautela in pazienti con marcata ipotensione arteriosa (PAS $<80$ $\mathrm{mmHg}$ ), elevati valori di creatininemia $(>3 \mathrm{mg} / \mathrm{dL}$ ), stenosi bilaterale delle arterie renali o elevati livelli di kaliemia $(>5.0 \mathrm{mEq} / \mathrm{L})$. Non si sono rilevate differenze significative in termini di miglioramento dei sintomi e della sopravvivenza tra $\mathrm{i}$ vari ACE-I attualmente disponibili. ${ }^{107} \mathrm{La}$ terapia va iniziata a basse dosi con graduale incremento della posologia se ben tollerata alle dosi minori; occorre, inoltre, effettuare un attento monitoraggio degli indici di funzionalità renale e della concentrazione sierica di $\mathrm{K}^{+}$.

L'uso dei diuretici è raccomandato nei pazienti con segni e sintomi di congestione, indipendentemente dalla FE, allo scopo di raggiungere e mantenere l'euvolemia (peso secco) con la minor dose di farmaco possibile, evitando il rischio di disidratazione. Nei pazienti ambulatoriali con $\mathrm{SC}$, la terapia diuretica viene generalmente iniziata con basse dosi, successivamente incrementate fino ad ottenere un aumento della diuresi ed una riduzione graduale del peso corporeo (pari a circa $0.5 / 1.0 \mathrm{~kg} / \mathrm{die})^{2,3}$

Gli $A R B s$ restano, ad oggi, un'alternativa nel trattamento dei pazienti intolleranti agli ACE- ${ }^{130}$ ma non sono più raccomandati come farmaci di prima scelta da introdurre nei pazienti con $\mathrm{HFrEF}$ ancora sintomatici nonostante un trattamento ottimale con ACE-I e BB. L'eplerenone ha dimostrato nello studio EMPHASIS$\mathrm{HF}^{131}$ di determinare una maggiore riduzione della morbilità e mortalità rispetto a quanto ottenuto in alcuni trials con l'aggiunta degli ARBs; inoltre, sempre nell'EMPHASIS-HF ${ }^{131}$ come pure nello studio RALES, ${ }^{132}$ il trattamento con MRA ha ridotto la mortalità per tutte le cause, a differenza dell'associazione con ARBs.

Gli MRA possono causare iperpotassiemia e peggioramento della funzionalità renale, effetti non comuni nei trials clinici randomizzati ma di frequente riscontro nella pratica clinica, specialmente in pazienti anziani e/o con alterata funzione renale. Attenzione va posta anche all'associazione con ACE-I. Andrebbero prescritti soltanto nei pazienti con adeguata funzionalità renale e valori normali di kaliemia, parametri da monitorare attentamente in corso di terapia.

Per ridurre il rischio di ospedalizzazione per SC 
nei pazienti con $\mathrm{FE} \leq 35 \%$ in ritmo sinusale e $\mathrm{FC} \geq 70$ $\mathrm{b} / \mathrm{m}$ ', con sintomatologia persistente (Classe NYHA II-IV) nonostante il trattamento con BB alla dose massima tollerata, ACE-I (o ARBs) ed un MRA ovvero in pazienti intolleranti ai $\mathrm{BB}$, viene considerato l'uso della ivabradina. ${ }^{133}$

La digossina può essere prescritta in aggiunta ad un ACE-I (o ARBs) e ad un MRA per ridurre il rischio di ospedalizzazione per SC nei pazienti, anche in ritmo sinusale, ancora sintomatici nonostante il trattamento con BB oppure intolleranti a questi. ${ }^{134}$ Diversi trials controllati con placebo hanno dimostrato che la digossina può migliorare la sintomatologia, la qualità di vita (valutata mediante lo score HRQOL) e la tolleranza all'esercizio in pazienti con SC lieve e moderato a prescindere dal ritmo, dall'eziologia dello SC e dalla terapia concomitante. ${ }^{135-141}$

L'idralazina e l'isosorbide dinitrato possono essere considerati un'alternativa agli ACE-I (o agli ARBs in caso di intolleranza), per ridurre il rischio di ospedalizzazione per SC o morte prematura nei pazienti con $\mathrm{FE} \leq 45 \%$ e dilatazione ventricolare sinistra (o $\mathrm{FE} \leq 35 \%$ ), in associazione ad un $\beta$-bloccante e ad un MRA, ${ }^{142,143}$ oppure in caso di persistenza della sintomatologia nonostante il trattamento con $\beta$-bloccante, ACE-I (o ARBs) e un MRA (o ARBs). ${ }^{144}$

Per ridurre ulteriormente il rischio di morte e di ospedalizzazione per SC può essere considerata l'aggiunta di $n-3$ PUFA al trattamento con ACE-I (o ARBs), BB e MRA (o ARBs). ${ }^{145}$

Secondo le più recenti linee guida sullo $\mathrm{SC}$ non vi è alcuna raccomandazione ad adoperare gli inibitori diretti della renina come alternativa agli ACE-I o ARB. ${ }^{3}$

Diversi trials clinici hanno dimostrato che i calcio antagonisti (CA) non hanno alcun effetto clinico favorevole o addirittura peggiorano la prognosi dei pazienti con SC..$^{146-150}$ I CA di prima generazione, non diidropiridinici, ma anche quelli diidropiridinici possiedono un'attività inotropa negativa. Nonostante la loro maggiore selettività per i canali del calcio della muscolatura liscia dei vasi, i CA di seconda generazione come l'amlodipina e la felodipina non hanno dimostrato alcun effetto benefico sulla funzionalità miocardica o sulla sopravvivenza nei pazienti con SC..$^{51-155}$ L'amlodipina può essere utilizzata nel trattamento dell'ipertensione arteriosa o della cardiopatia ischemica nei pazienti con $\mathrm{SC}$, in quanto ben tollerata e dotata di effetti neutri sulla morbidità e mortalità. La maggior parte dei CA, quindi, ad eccezione dell'amlodipina e della felodipina, non andrebbero adoperati nei pazienti con SC e frazione di eiezione ridotta per la loro azione inotropa negativa in grado di peggiorare lo SC. . $^{147,156}$

Quasi tutti i risultati di ampi trials randomizzati controllati hanno dimostrato che i farmaci antiaritmici aumentano la mortalità nei pazienti con SC. ${ }^{157-159}$ Soltanto l'amiodarone e la dofetilide hanno mostrato nei trials clinici di non influire sulla mortalità e sono quindi da preferire nel trattamento delle aritmie nei pazienti con SC. . $^{160-163}$

Pur presentando i pazienti con $\mathrm{HFrEF}$ un rischio trombo-embolico aumentato, ${ }^{164-166}$ con la eccezione dei pazienti con fibrillazione atriale, non vi è alcuna evidenza in questi soggetti che gli anticoagulanti orali riducano la morbilità o la mortalità, rispetto al placebo o all'aspirina (ASA). ${ }^{167}$

L'utilizzo dell' $A S A$ è utile nei pazienti con SC secondario a cardiopatia ischemica (prima causa di SC), ma analisi retrospettive e studi prospettici ne hanno evidenziato un aumento della morbilità nella altre forme di SC. In un recente studio, l'utilizzo di basse dosi di ASA ha migliorato l'outcome (riducendo morbilità e mortalità) nei pazienti con SC senza differenze tra i pazienti che assumevano dosi di aspirina $>75$ $\mathrm{mg} /$ die o che non l'assumevano. ${ }^{168}$

Diversamente dalle evidenze presenti per altre patologie cardiologiche, i dati della letteratura non sono a supporto dell'uso delle statine nei pazienti con SC cronico. ${ }^{169,170}$

Un nuovo farmaco, un'associazione di sacubitril (inibitore della neprilisina) con il valsartan, ha dimostrato in pazienti con $\mathrm{HFrEF}$ la sua superiorità rispetto all'enalapril nel ridurre il rischio di morte cardiovascolare e per tutte le cause e di ospedalizzazione per SC. Questo nuovo farmaco potrebbe rappresentare il primo in grado di sconvolgere il precedente algoritmo del trattamento dello SC, rimasto fondamentalmente invariato nell'ultimo decennio. ${ }^{171,172}$

Per quanto concerne specificatamente lo $\mathrm{HF} p \mathrm{EF}$, nessun trattamento farmacologico ha dimostrato finora, in maniera incontrovertibile, di ridurne la morbilità e la mortalità.

I diuretici sono utilizzati per controllare la congestione (vanno tuttavia somministrati con cautela, a dosi non elevate, per evitare una eccessiva riduzione del pre-carico).

È importante il trattamento dell'ipertensione arteriosa e dell'ischemia miocardica così come il controllo della frequenza ventricolare nei pazienti con fibrillazione atriale (FA).

Pochi studi molto limitati hanno dimostrato che il verapamil, CA non diidropiridinico con azione cronotropa negativa, può migliorare la tolleranza all'esercizio ed i sintomi nei pazienti con HF $p$ EF. ${ }^{173,174}$ I CA cronotropi negativi potrebbero essere utili anche per il controllo della frequenza ventricolare nei pazienti con FA ed in trattamento per ipertensione arteriosa e/o ischemia miocardica (a differenza dei pazienti con $\mathrm{HF} r \mathrm{EF}$ nei quali l'effetto inotropo negativo di questi farmaci potrebbe risultare dannoso).

Anche i BB possono essere utilizzati per il controllo della frequenza ventricolare nei pazienti con $\mathrm{HF} p \mathrm{EF}$ e FA. 
Nei pazienti con HFpEF vanno evitati gli stessi farmaci ritenuti dannosi nei pazienti con $\mathrm{HFrEF}$, ad eccezione, quindi, dei CA.

Le terapie antineoplastiche hanno, in diversi casi, trasformato il cancro in una malattia cronica facendo emergere gli effetti collaterali cardiovascolari di alcune terapie antitumorali (antracicline, come la doxorubicina, 5-fluorouracile, il suo profarmaco capecitabina ed il trastuzumab ${ }^{175}$ ), talora precocemente, ma anche diversi anni dopo la sospensione del farmaco, con comparsa di SC con disfunzione sistolica prevenibile con l'uso precoce di BB e/o ACE-I.

La sideropenia che può contribuire alla disfunzione miocardica nei pazienti con $\mathrm{HFrEF}$ e determinare uno stato anemico, andrebbe adeguatamente trattata. È stato, infatti, dimostrato che dopo sei mesi di terapia marziale, si assiste ad un miglioramento soggettivo delle condizioni generali del paziente, sia in termini di cenestesi che di classe NYHA. ${ }^{176}$ Sia il FAIR-HF che il CONFIRM-HF hanno recentemente confermato questi dati con la supplementazione endovenosa (e.v.) di ferro-carbossimaltosio in pazienti con $\mathrm{HFrEF}$, dimostrando anche che la somministrazione e.v. è meglio tollerata rispetto alla via orale. ${ }^{177}$ Non sono ancora conosciuti, invece, gli effetti della terapia marziale nei pazienti con $\mathrm{HF} p$ EF e la sua sicurezza nel lungo termine.

Per quanto concerne il diabete mellito, alcuni farmaci adoperati nella cura dello SC hanno dimostrato un effetto preventivo su questa importante comorbidità, come ad esempio gli ARBs e probabilmente gli ACE-I. ${ }^{178} \mathrm{I} \mathrm{BB}$, hanno dimostrato di migliorare la prognosi nei pazienti diabetici con $\mathrm{SC}$, anche se va precisato che differenti BB possono avere effetti diversi sugli indici glicemici. ${ }^{179}$ I glitazoni, invece, vanno evitati, per l'aumentato rischio di peggioramento dello $\mathrm{SC}$ ed ospedalizzazione per la loro azione di ritenzione idro-salina. ${ }^{180-182}$ La metformina è controindicata nel caso di insufficienza renale o epatica per il rischio di acidosi lattica. ${ }^{183}$

\section{Devices e procedure di cardio-chirurgia}

L'indicazione all'impianto di un devices o a procedure di cardio-chirurgia non è compito né competenza del medico internista, il quale tuttavia deve conoscere le relative indicazioni e saper attivare le consulenze specialistiche del caso.

\section{Defibrillatore impiantabile (ICD)}

La metà dei decessi nei pazienti con SC avviene in concomitanza con un'aritmia ventricolare maligna ed in misura minore per bradiaritmia o asistolia. I farmaci antiaritmici non riducono il rischio di morte, anzi talora lo aumentano per comparsa di effetti proaritmici. L'impianto di un device del tipo $\mathrm{ICD}^{184}$ è indicato in prevenzione primaria (pazienti con $\mathrm{FE} \leq 35 \%$, con cardiopatia dilatativa ischemica o non ischemica, in classe NYHA II-III sotto trattamento farmacologico massimale) ovvero in prevenzione secondaria ${ }^{185,186}$ (pazienti sopravvissuti ad un primo episodio di arresto cardiaco o ad aritmia ventricolare sintomatica, indipendentemente dalla $\mathrm{FE}$, purchè in soddisfacente stato funzionale e con aspettativa di vita $>1$ anno). La terapia antiaritmica con amiodarone non riduce la mortalità in questi pazienti. ${ }^{187}$

\section{Terapia di resincronizzazione cardiaca (RCT)}

L'impianto di un device del tipo RCT, ${ }^{184}$ con o senza $\mathrm{ICD}$ e/o funzione di pace-maker, è efficace nel migliorare la prognosi dei pazienti con $\mathrm{SC}$ in ritmo sinusale, con QRS di durata $>130 \mathrm{~ms}$ e blocco di branca sinistro, $\mathrm{FE} \leq 35 \%$, purchè in buono stato funzionale, con sopravvivenza prevista superiore ad 1 anno. ${ }^{188-191}$

\section{Intervento di rivascolarizzazione coronarica (sia per via percutanea che cardio-chirurgica)}

È indicato in caso di angina pectoris persistente o refrattaria nonostante trattamento farmacologico appropriato, in presenza di cardiopatia ischemica severa; le indicazioni sono di competenza del cardio-chirurgo o del cardiologo interventista dopo valutazione collegiale nell'ambito di uno Heart Team; ${ }^{192}$ analogo approccio integrato è da seguire per una indicazione alla chirurgia valvolare (vedi recenti linea guida europee). ${ }^{193}$

\section{Trapianto di cuore}

Viene abitualmente preso in considerazione in presenza di uno SC avanzato, refrattario alle terapie convenzionali. ${ }^{194,195}$ Indicazioni al trapianto di cuore sono: sintomatologia severa nonostante terapia ottimizzata; prognosi sfavorevole a breve termine; condizioni generali ancora discrete; assenza di alternative terapeutiche; motivazione del paziente dopo adeguata informazione; assenza di controindicazioni. Controindicazioni al trapianto di cuore sono: comorbilità (neoplasia trattata nei 5 anni precedenti, insufficienza renale o epatica, malattie sistemiche multi-organo, malattie psichiatriche); elevate resistenze vascolari polmonari; abuso di alcool o droghe.

\section{Gestione dello scompenso cardiaco}

Lo SC presenta periodiche riacutizzazioni ed è ulteriormente aggravato dalle comorbilità associate e dall'età avanzata, ciò spiega perché siano così frequenti ricoveri ospedalieri ripetuti.

Nello studio Temistocle ${ }^{29}$ il $45 \%$ dei pazienti veniva ricoverato entro 6 mesi dal primo ricovero. In uno studio Medicare su 1.330.157 pazienti il re-ricovero ad un mese 
avveniva nel $24,8 \%$ ma con una frequenza del $61 \%$ entro i primi $15 \mathrm{gg}$ dalle dimissioni. ${ }^{196,197} \mathrm{In}$ un recente studio retrospettivo i re-ricoveri ad un anno per SC risultavano del $67,4 \%$ con una mortalità del $28,7 \% .{ }^{198}$

Il tasso di riospedalizzazione per SC è considerato come uno degli indicatori della qualità delle cure $\mathrm{e}$ della competenza dei diversi centri. ${ }^{197}$ Le cause di ricovero ripetuto, possono essere ricondotte ad una causa cardiaca (progressione della cardiopatia di base, IMA, ipertensione arteriosa non controllata, ischemia miocardica o aritmie) o non cardiaca (BPCO, anemia, infezioni, tireopatie), a scarsa compliance terapeutica, ad errori dietetici (eccessiva introduzione di liquidi, eccesso di sodio nella dieta, fumo di sigaretta, eccesso di alcol), ma una quota rilevante è collegata ad eventi avversi da farmaci (FANS, steroidi, antracicline ed altri chemioterapici, calcio-antagonisti non diidropiridinici). Un rilievo notevole sembra avere lo stato socio-economico ed il livello di scolarità. ${ }^{199}$

Da tutti questi dati è emersa la convinzione che molte delle riospedalizzazioni sono prevenibili, ma i modelli in grado di predire le riospedalizzazioni sono generalmente inadeguati, spesso non tengono conto di molti fattori ambientali e sociali, e non sono sempre in grado di caratterizzare esattamente la severità della malattia e le comorbilità ${ }^{200}$ Per prevenire le riospedalizzazioni sono stati sperimentati modelli di continuità tra ospedale e territorio con ambulatori dedicati, a gestione medica/infermieristica o modelli con assistenza domiciliare; questi programmi hanno dato buoni risultati, tuttavia con un rapporto costo beneficio non documentato. Gonseth ${ }^{201}$ ha evidenziato dati positivi in quasi tutte le esperienze di continuità ospedale territorio riportate in vari studi.

Il controllo a distanza di questi pazienti potrebbe aggirare il problema della loro difficoltà di movimento. La telemedicina con il monitoraggio a distanza, la teleassistenza e il teleconsulto sono stati campi di sperimentazione. Evidenze di moderata qualità mostrano che il telemonitoraggio riduce la mortalità e le ospedalizzazioni per tutte le cause, ma non migliora la qualità di vita né diminuisce le ospedalizzazioni per SC. I dati della letteratura sono molto disomogenei e le linee guida $\mathrm{NICE}^{202}$ non includono tra le raccomandazioni il telemonitoraggio, anche se potenzialmente efficace. Il monitoraggio a lungo termine rimane un'ipotesi affascinante, ma richiede un notevole dispendio di risorse. Evidenze di moderata qualità mostrano una lieve riduzione delle ospedalizzazioni nel medio-lungo termine (9-15 mesi) con una terapia guidata dal BNP, ma non una riduzione della mortalità; inoltre misure riabilitative tendono a ridurre le ospedalizzazioni per SC nei pazienti con bassa $\mathrm{FE}^{203}$

L'evoluzione tecnologica ha fornito dispositivi invasivi di controllo a distanza, come l'impedenza intratoracica, che forniscono informazioni impor- tanti ${ }^{204,205}$ anche nello SC con FE conservata. ${ }^{206}$ Tuttavia, come per tutti gli strumenti diagnostici, gli studi per valutarne l'efficacia sono di difficile progettazione e la corretta interpretazione delle modificazioni dei segnali di impedenza rimane una sfida difficile nella pratica clinica. ${ }^{207}$

Ciò che oggi è fattibile isorisorse, considerando anche il numero rilevante di pazienti affetti da SC ed il loro incremento nel tempo, è rivedere ciò che abitualmente viene, o non viene fatto, e adeguarci a uno specifico programma comprendente: i) un approccio di team multidisciplinare la cui composizione è correlata alle risorse tecniche ed economiche disponibili in ospedale e sul territorio; ii) un piano di continuità stabilito già durante il ricovero; iii) un accesso facilitato alla diagnostica ed al trattamento specialistico; iv) l'ottimizzazione della terapia medica secondo le linee guida, ma centrato sul singolo paziente secondo le eventuali comorbilità; v) un programma di educazione del paziente per ridurre le difficoltà di aderenza alla terapia e, laddove possibile, l'educazione di una figura di sostegno; vi) una precoce attenzione ai segni e ai sintomi; vii) un regime flessibile nell'uso dei diuretici, eventualmente auto-gestito; viii) un follow-up attento ed una prima visita programmata a breve termine dopo la dimissione dall'ospedale; ix) individuazione della popolazione a rischio elevato di riospedalizzazione, prevedendo un piano di gestione specifico.

L'ipotesi di introdurre penalizzazioni di ordine finanziario per gli ospedali con elevato tasso di riospedalizzazione (così come proposto nei pazienti Medicare) appare confondente in quanto rischierebbe di penalizzare le strutture ospedaliere che si occupano dei pazienti più gravi o in condizioni socioeconomiche più disagiate. ${ }^{208}$

In una realtà come quella del Sistema Sanitario Nazionale italiano i fattori che incidono sul tasso di riospedalizzazione vanno riferiti non solo alla realtà ospedaliera, ma anche alle risorse ed agli aspetti organizzativi presenti sul territorio.

\section{Indicatori di qualità}

Nel percorso gestionale dei pazienti con SC si raccomanda l'individuazione di specifici indicatori di qualità (indicatori di struttura, di processo e di risultato), ${ }^{209-214}$ condivisi a livello ospedaliero e territoriale, utili per attivare processi di autovalutazione e di miglioramento continuo della qualità dell'assistenza, nonché per individuare i bisogni formativi emergenti.

\section{Indicatori di struttura}

i) Accesso a linee guida aggiornate e percorsi diagnostico-terapeutici condivisi;

ii) Monitoraggio dell'assistenza erogata e degli esiti; 
iii) Elaborazione di programmi di gestione assistenziale;

iv) Presenza di ambulatori specialistici dedicati;

v) Definizione di percorsi facilitati verso centri ad alta specializzazione ove necessari.

\section{Indicatori di processo}

i) Assessment clinico-strumentale (ricerca della eziologia della cardiopatia e dei fattori scatenanti; valutazione della funzione sistolica del ventricolo sinistro);

ii) Prescrizione di farmaci appropriati, a dosi appropriate, purchè non controindicati;

iii) Prevenzione del rischio tromboembolico con anticoagulanti orali, se non controindicati, nei pazienti con SC e FA;

iv) Educazione sanitaria dei pazienti e loro familiari;

v) Valutazione multidimensionale dei pazienti sopra i 65 anni: stato fisico, capacità cognitive, benessere psichico, livello di autosufficienza;

vi) Compilazione di lettera dettagliata di dimissione (Tabella 1).

\section{Indicatori di esito}

i) Ricoveri ripetuti e/o numero di accessi in PS/DEA a 30 ed a 90 giorni;

ii) Tasso di mortalità (intraospedaliera ed a 30 giorni dalla dimissione);

iii) Consumo di risorse sanitarie (tasso di riospedalizzazione, accessi al Pronto Soccorso, visite specialistiche):

iv) Capacità del paziente a cogestire la malattia, qualità di vita e soddisfazione dei pazienti.

\section{Conclusioni}

Rispetto al quesito 1: Quali modalità di valutazione del rischio e di stratificazione prognostica (anche rispetto alle comorbilità esistenti)? riteniamo di dover rispondere al quesito riportando le seguenti condizioni cliniche associate a prognosi sfavorevole:

- Aspetti clinici: età avanzata, cardiopatia ischemica o ipertensiva, classe NYHA III o IV, scarsa compliance, riospedalizzazioni frequenti, morte improvvisa rianimata;

- Comorbilità: diabete mellito, BPCO e OSAS, insufficienza renale, anemia, depressione, cachessia, valvulopatia mitralica ed aortica;

- Elettrocardiogramma: tachicardia sinusale, fibrillazione atriale, blocco di branca sinistro, onde Q, ipertrofia ventricolare sinistra, aritmie maggiori;

- Ecocardiogramma: ridotta frazione di eiezione, dilatazione dei volumi del ventricolo sinistro, pattern mitralico restrittivo, ridotta funzione del ventricolo destro, ipertensione polmonare;

- Esami di laboratorio: aumento dei seguenti parametri fattori natriuretici (BNP), Tn, creatininemia e/o azotemia, enzimi epatici, uricemia e riduzione di natriemia ed emoglobina

Parametri funzionali: ridotto picco VO2 (volume massimo di consumo di ossigeno nell'unità di tempo), riduzione del test del cammino per 6 minuti (6 min WT), respiro periodico. ${ }^{62}$

Alla domanda n. 2: Quali azioni da implementare, ritagliate sul singolo paziente? riteniamo di rispondere con quanto riportato in Tabella 2.

Tabella 1. Lettera di dimissione (strumento cardine della continuità assistenziale).

Motivo del ricovero, cause di instabilizzazione, modalità di presentazione clinica

Diagnosi eziologica, presenza di comorbilità, stratificazione del rischio

Dati di laboratorio, esami strumentali, consulenze specialistiche

Terapie farmacologiche prescritte durante il ricovero e consigliate alla dimissione

Peso secco, frequenza cardiaca, pressione arteriosa alla dimissione

Programma di follow-up a breve termine (entro 7-10 giorni) ed a medio-lungo termine

Supporto infermieristico ed assistenza sociale a domicilio; contatti telefonici preferenziali

Educazione sanitaria del paziente e familiari per la autogestione

Mathieu G, comunicazione personale. 
Tabella 2. Azioni da implementare per la prevenzione nel singolo paziente.

Il paziente con SC deve essere reso consapevole della sua patologia attraverso un programma educazionale che lo aiuti a migliorare la cura di sé e la propria qualità di vita, prevenendo le riacutizzazioni della malattia e migliorandone la prognosi, in stretta collaborazione con il proprio medico

Interventi non farmacologici irrinunciabili sono costituiti dalle misure dietetiche e comportamentali, come la dieta iposodica, la riduzione dell'introito di liquidi, il training fisico e l'abolizione del fumo di sigaretta

Particolare attenzione va posta alle comorbidità, come le turbe del sonno (OSAS), l'obesità ma anche la cachessia; occorre inoltre valutare attentamente gli effetti collaterali di alcuni farmaci di uso comune (FANS in particolare) o meno comune

Va incoraggiata una vita attiva, coltivando i rapporti sociali e la cura del proprio tempo libero con massima attenzione ai primi segni e sintomi di una eventuale depressione

Di fondamentale importanza sono gli interventi farmacologici, volti a migliorare la sintomatologia legata a congestione e/o bassa portata, a prevenire le ospedalizzazioni ed a ridurre la mortalità

In particolare, nei pazienti con $\mathrm{HFrEF}$, gli ACE inibitori (o gli antagonisti recettoriali dell'angiotensina), i $\beta$-bloccanti e gli anti aldosteronici sono le classi di farmaci antagonisti neuro-umorali di importanza basilare nel modificare il decorso e la prognosi dello SC

Un regime flessibile nell'uso dei diuretici (eventualmente autogestito) viene utilizzato per ridurre segni e sintomi di congestione

La terapia va iniziata, con la maggior parte dei farmaci, a basse dosi con successivo progressivo e graduale incremento fino alla massima posologia indicata dalle linee guida e tollerata dal paziente

Molta attenzione deve essere posta all'interazione tra i vari farmaci adoperati (in particolare l'azione sugli elettroliti) anche in considerazione di alcune possibili comorbidità (come ad esempio l'insufficienza renale)

Peculiare comportamento terapeutico va adottato per quanto concerne lo $\mathrm{HF} p \mathrm{EF}$, per il quale nessun trattamento farmacologico ha dimostrato finora, in maniera incontrovertibile, di ridurne la morbilità e la mortalità

In particolare è opportuno seguire le seguenti avvertenze: cautela nella somministrazione dei diuretici per evitare una riduzione del pre-carico; riduzione della frequenza cardiaca per favorire il riempimento ventricolare, trattamento appropriato dell'ipertensione arteriosa e della cardiopatia ischemica

SC, scompenso cardiaco; OSAS, sindrome da apnee ostruttive nel sonno; FANS, farmaci anti-infiammatosi non steroidei; HFrEF, frazione d'eiezione ridotta; HF $p$ EF, frazione d'eiezione conservata.

\section{Bibliografia}

1. Braunwald E. Heart failure: an overview. New York: Mc Graw Hill; 1997.

2. McMurray JJP, Adamopoulos S, Anker SD, et al. ESC guidelines for the diagnosis and treatment of acute and chronic heart failure 2012: the Task Force for the Diagnosis and Treatment of Acute and Chronic Heart Failure 2012 of the European Society of Cardiology. Eur Heart J 2012;33:1787-847.

3. Yancy CW, Jessup M, Bozkurt B, et al. 2013 ACCF/AHA Guideline for the management of heart failure: a report of the American College of Cardiology Foundation/American Heart Association Task Force on Practice Guidelines. J Am Coll Cardiol 2013 [Epub ahead of print].

4. Fonarow GC, Stough WG, Abraham WT, et al. Characteristics, treatments, and outcomes of patients with preserved systolic function hospitalized for heart failure: a report from the OPTIMIZE-HF Registry. J Am Coll Cardiol 2007;50:768-77.

5. The Criteria Committee of the New York Heart Associ- ation. Nomenclature and criteria for diagnosis of diseases of the heart and great vessels. 9th ed. Boston, MA: Little \& Brown; 1994.

6. Goldman L, Hashimoto B, Cook EF, et al. Comparative reproducibility and validity of systems for assessing cardiovascular functional class: advantages of a new specific activity scale. Circulation 1981;64:1227-34.

7. Madsen BK, Hansen JF, Stokholm KH, et al. Chronic congestive heart failure: description and survival of 190 consecutive patients with a diagnosis of chronic congestive heart failure based on clinical signs and symptoms. Eur Heart J 1994;15:303-10.

8. Mosterd A, Hoes AW. Clinical epidemiology of heart failure. Heart 2007;93:1137-46.

9. Owan TE, Hodge DO, Herges RM, et al. Trends in prevalence and outcome of heart failure with preserved ejection fraction. N Engl J Med 2006;355:251-9.

10. Hogg K, Swedberg K, McMurray JJP. Heart failure with preserved left ventricular systolic function; epidemiology, clinical characteristics, and prognosis. J Am Coll Cardiol 2004;43:317-27.

11. Lam CS, Donal E, Kraigher-Krainer E, Vasan RS. Epi- 
demiology and clinical course of heart failure with preserved ejection fraction. Eur J Heart Fail 2011;13:18-28.

12. Meta-analysis Global Group in Chronic Heart Failure (MAGGIC). The survival of patients with heart failure with preserved or reduced left ventricular ejection fraction: an individual patient data meta-analysis. Eur Heart J 2012;33:1750-57.

13. Remes J, Miettinen H, Reunanen A, et al. Validity of clinical diagnosis of heart failure in primary health care. Eur Heart J 1991;12:315-21.

14. Wheeldon NM, MacDonald TM, Flucker CJ, et al. Echocardiography in chronic heart failure in the community. Q J Med 1993;86:17-23.

15. Lloyd-Jones D, Adams RJ, Brown TM, et al. Heart disease and stroke statistics 2010 Update. Circulation 2010;121:e46-215.

16. Stewart S, Jenkins A, Buchan S, et al. The current cost of heart failure to the National Health Service in the UK. Eur J Heart Fail 2002;4:361-71.

17. Dunlay SM, Shah ND, Shi Q, et al. Lifetime costs of medical care after heart failure diagnosis. Circ Cardiovasc Qual Outcomes 2011;4:68-75.

18. Murdoch DR, Love MP, Robb SD, et al. Importance of heart failure as a cause of death. Changing contribution to overall mortality and coronary heart disease mortality in Scotland 1979-1992. Eur Heart J 1998;19:1829-35.

19. MacIntyre K, Capewell S, Stewart S, et al. Evidence of improving prognosis in heart failure: trends in case fatality in 66547 patients hospitalized between 1986 and 1995. Circulation 2000;102:1126-31.

20. Blackledge HM, Tomlinson J, Squire IB. Prognosis for patients newly admitted to hospital with heart failure: survival trends in 12220 index admissions in Leicestershire 1993-2001. Heart 2003;89:615-20.

21. Schaufelberger M, Swedberg K, Koster M, et al. Decreasing one-year mortality and hospitalization rates for heart failure in Sweden; data from the Swedish Hospital Discharge Registry 1988 to 2000. Eur Heart J 2004; 25:300-7.

22. Cowie MR, Wood DA, Coats AJ, et al. Survival of patients with a new diagnosis of heart failure: a population based study. Heart 2000;83:505-10.

23. Stewart S, MacIntyre K, Hole DJ, et al. More 'malignant' than cancer? Five-year survival following a first admission for heart failure. Eur J Heart Fail 2001;3:315-22.

24. Stewart S, Ekman I, Ekman T, et al. Population impact of heart failure and the most common forms of cancer: a study of 1,162,309 hospital cases in Sweden (1988 to 2004). Circ Cardiovasc Qual Outcomes 2010;3:573-80.

25. Askoxylakis V, Thieke C, Pleger ST, et al. Long-term survival of cancer patients compared to heart failure and stroke: a systematic review. BMC Cancer 2010;10:105.

26. Roger VL. The heart failure epidemic. Int J Environ Res Public Health 2010;7:1807-30.

27. Steinberg BA, Zhao X, Heidenreich PA, et al. Trends in patients hospitalized with heart failure and preserved left ventricular ejection fraction: prevalence, therapies, and outcomes. Circulation 2012;126:65-75.

28. Task Force for the Diagnosis and Treatment of Acute and Chronic Heart Failure. ESC guidelines for the diagnosis and treatment of acute and chronic heart failure 2012. Eur Heart J 2012;33:1787-847.

29. Di Lenarda A, Scherillo M, Maggioni AP, et al. Current presentation and management of heart failure in cardiology and internal medicine hospital unit. A tale of two worlds. Am Heart J 2003;146:735.

30. Biagi P, Gussoni G, Iori I, et al. Clinical profile and predictors of in-hospital outcome in patients with heart failure: The FADOI "CONFINE" Study. Int J Cardiol 2011;152:88.

31. Biagi P, Nardi R, Baldo IC, et al. Clinical characteristics of very old patients hospitalized in internal medicine wards for heart failure: a subanalysis of the FADOI CONFINE Study Group. Ital J Med 2014;8:19-28.

32. Felker GM, Thompson, Joshua MH, et al. Underlying causes and long term survival in patients with initially unexplained cardiomiopathy. NEJM 2000;342:1077-84.

33. Zile MR, Brutsaert DL. New concepts in diastolic or non diastolic HF. Circulation 2002;105:1387-93.

34. Komajda M, Hanon O, Hocadel M, et al. Management of octuagenarians hospitalized for heart failure in Euro Eart failure Survey. Eur Heart J 2006;28:1310-8.

35. Gheorghiade M, Abraham WR, Albert NM, et al. Systolic blood pressure and admission clinical characteristics and outcomes in patients hospitalized with Heart Failure. JAMA 2006;296:2217-26.

36. Wang TJ, Gona P, Larson MG, et al. Multiple biomarkers for the prediction of first major cardiovascular events and death. N Engl J Med 2006;355:2631-9.

37. Latini R, Masson S, Anand IS, et al. Prognostic value of very low plasma concentrations of troponin $\mathrm{T}$ in patients with stable chronic heart failure. Circulation 2007;116: 1242-9.

38. Gheorghiade M, Rossi JS, Cotts W, et al. Characterization and prognostic value of persistent hyponatremia in patients with severe heart failure in the ESCAPE Trial. Arch Int Med 2007;167:1998-2005.

39. Baldasseroni S, Opasich C, Gorini M, et al. Left bundlebranch block is associated with increased 1-year sudden and total mortality rate in 5517 outpatients with congestive heart failure: A report from the Italian Network on Congestive Heart Failure. Am Heart J 2002;143:398-405.

40. Benjamin EJ, Wolf PA, D'Agostino RB, et al. Impact of atrial fibrillation on the risk of death: the Framingham Heart Study. Circulation 1998;98:946-52.

41. Hillege HL, Girbes ARJ, de Kam PJ, et al. Renal function, neurohormonal activation, and survival in patients with chronic heart failure. Circulation 2000;102:203-10.

42. Hillege HL, Nitsch D, Pfeffer MA, et al. Renal function as a predictor of outcome in a broad spectrum of patients with heart failure. Circulation 2006;113:671-8.

43. Smilde TDJ, van Veldhuisen DJ, Navis G, et al. Drawbacks and prognostic value of formulas estimating renal function in patients with chronic heart failure and systolic dysfunction. Circulation 2006;114;1572-80.

44. Jackson CE, Solomon SD, Gerstein HC, et al. Albuminuria in chronic heart failure: prevalence and prognostic importance. Lancet 2009;374:543-50.

45. Rusinaru D, Saaidi I, Godard S, et al. Impact of COPD on longterm outcome of patients hospitalized for heart failure. Am J Cardiol 2008;101:353-8.

46. Macchia A, Monte S, Romero M, et al. The prognostic influence of chronic obstructive pulmonary disease in patients hospitalised for chronic heart failure. Eur $\mathrm{J}$ Heart Fail 2007;9:942-8.

47. Holguin F, Folch E, Red SC, et al. Comorbidity and 
mortality in COPD-related hospitalizations in the United States, 1979 to 2001. Chest 2005;128;2005-11.

48. Sidney S, Sorel M, Quesenberry CP, et al. COPD and incident cardiovascular disease hospitalizations and mortality: Kaiser Permanente Medical Care Program. Chest 2005;128:2068-75.

49. Wang H, Parker JD, Newton GE, et al. Influence of obstructive sleep apnea on mortality in patients with heart failure. J Am Coll Cardiol 2007;49:1625-31.

50. Anand IS, McMurray JJV, Whitmore J, et al. Anemia and its relationship to clinical outcome in heart failure. Circulation 2004;110:149-54.

51. O’Meara E, Clayton T, McEntegart MB, et al. Clinical correlates and consequencies of anemia in a broad spectrum of patients with heart failure. results of the candesartan in heart failure: assessment of reduction in mortality and morbidity (CHARM) program. Circulation 2006;113:986-94.

52. Groenveld HF, Jannuzzi JL, Damman K, et al. Anemia and mortality in heart failure patients. A systematic review and meta analysis. J Am Coll Cardiol 2008;52:818-27.

53. MacDonald MR, Petrie MC, Hawkins MN, et al. Diabetes, left ventricular systolic dysfunction and chronic heart failure. Eur Heart J 2008;29:1224-49.

54. Haas SJ, Vos T, Gilbert RE, et al. Are beta-blockers as efficacious in patients with diabetes mellitus as in patients without diabetes mellitus who have chronic heart failure? A metaanalysis of large-scale clinical trials. Am Heart J 2003;146:848-53.

55. Gerstein HC, Swedberg K, Carlsson J, et al. The HbA1c levels as a progressive risk factor for cardiovascular death, hospitalization for heart failure or death in patients with chronic heart failure. JAMA 2008;168:1699-704.

56. De Denus S, Spinler SA, Jessup M, et al. History of depression as a predictor of adverse outcomes in patients hospitalized for decompensated heart failure. Pharmacotherapy 2004;24:1306-10.

57. Sherwood A, Blumenthal JA, Trivedi T, et al. Relationship of depression to death ot hospitalization in patients with heart failure. Arch Intern Med 2007;167:367-73.

58. Anker SD, Sharma T. The syndrome of cardiac cachexia. Int J Cardiol 2002;85:51-66.

59. Springer J, Filippatos G, Akashi A, et al. Prognosis and therapy approaches of cardiac cachexia. Curr Opin Cardiol 2006;21:229-33.

60. Gheorghiade M, Zannad F, Sopko G, et al. Acute heart failure syndromes. Current state and framework for future research. Circulation 2005;112:3958-68.

61. Nohria A, Lewis E, Stevenson LW. Medical management of advanced heart failure. JAMA 2002;287:628-40.

62. Dickstein K, Cohen-Solal A, Filippatos G, et al. ESC Guidelines for the diagnosis and treatment of acute and chronic heart failure 2008. Eur Heart J 2008;29:2388-442.

63. Boren SA, Wakefield BJ, Gunlock TL, et al. Heart failure self-management education: a systematic review of the evidence. Int J Evid Based Healthc 2009;7:159-68.

64. Gwadry-Sridhar FH, Arnold JM, Zhang Y, et al. Pilot study to determine the impact of a multidisciplinary educational intervention in patients hospitalized with heart failure. Am Heart J 2005;150:982.

65. Koelling TM, Johnson ML, Cody RJ, et al. Discharge education improves clinical outcomes in patients with chronic heart failure. Circulation 2005;111:179-85.
66. VanSuch M, Naessens JM, Stroebel RJ, et al. Effect of discharge instructions on readmission of hospitalised patients with heart failure: do all of the Joint Commission on Accreditation of Healthcare Organizations heart failure core measures reflect better care? Qual Saf Health Care 2006;15:414-7.

67. Aguado O, Morcillo C, Delas J, et al. Long-term implications of a single home-based educational intervention in patients with heart failure. Heart Lung 2010;39:S14-22.

68. Wingham J, Frost J, Britten N, et al. Needs of caregivers in heart failure management: A qualitative study. Chronic Illn 2015;pii:1742395315574765 [Epub ahead of print].

69. Riegel B, Moser DK, Anker SD, et al. State of the science: promoting self-care in persons with heart failure: a scientific statement from the American Heart Association. Circulation 2009;120:1141-63.

70. Taylor RS, Sagar VA, Davies EJ, et al. Exercise-based rehabilitation for heart failure. Cochrane Database Syst Rev 2014;(4):CD003331.

71. McKelvie RS. Exercise training in patients with heart failure: clinical outcomes, safety, and indications. Heart Fail Rev 2008;13:3-11.

72. Pina IL, Apstein CS, Balady GJ, et al. Exercise and heart failure: a statement from the American Heart Association Committee on Exercise, Rehabilitation, and Prevention. Circulation 2003;107:1210-25.

73. Piepoli MF, Davos C, Francis DP, et al. Exercise training meta-analysis of trials in patients with chronic heart failure (ExTraMATCH). BMJ 2004;328:189.

74. Austin J, Williams R, Ross L, et al. Randomised controlled trial of cardiac rehabilitation in elderly patients with heart failure. Eur J Heart Fail 2005;7:411-7.

75. Austin J, Williams WR, Ross L, et al. Five-year followup findings from a randomized controlled trial of cardiac rehabilitation for heart failure. Eur J Cardiovasc Prev Rehabil 2008;15:162-7.

76. Gallager R, Luttik ML, Jaarsma T. Social support and selfcare in heart failure. J Cardiovasc Nurs 2011;26:439-45.

77. Luttik ML, Jaarsma T, Moser D, et al. The importance and impact of social support on outcomes in patients with heart failure: an overview of the literature. J Cardiovasc Nurs 2005;20:162-9.

78. Struthers AD, Anderson G, Donnan PT, et al. Social deprivation increases cardiac hospitalisations in chronic heart failure independent of disease severity and diuretic non-adherence. Heart 2000;83:12-6.

79. Murberg TA, Bru E. Social relationships and mortality in patients with congestive heart failure. J Psychosom Res 2001;51:521-7.

80. Murberg TA. Long-term effect of social relationships on mortality in patients with congestive heart failure. Int $\mathrm{J}$ Psychiatry Med 2004;34:207-17.

81. Lennie TA, Song EK, Wu JR, et al. Three gram sodium intake is associated with longer event-free survival only in patients with advanced heart failure. J Card Fail 2011;17:325-30.

82. Arcand J, Ivanov J, Sasson A, et al. A high-sodium diet is associated with acute decompensated heart failure in ambulatory heart failure patients: a prospective followup study. Am J Clin Nutr 2011;93:332-7.

83. Cody RJ, Covit AB, Schaer GL, et al. Sodium and water balance in chronic congestive heart failure. J Clin Invest 1986;77:1441-52. 
84. Cook NR, Cutler JA, Obarzanek E, et al. Long term effects of dietary sodium reduction on cardiovascular disease outcomes: observational follow-up of the Trials of Hypertension Prevention (TOHP). BMJ 2007;334: 885-8.

85. Paterna S, Gaspare P, Fasullo S, et al. Normal-sodium diet compared with low-sodium diet in compensated congestive heart failure: is sodium an old enemy or a new friend? Clin Sci (Lond) 2008;114:221-30.

86. Basuray A, Dolansky M, Josephson R, et al. Dietary sodium adherence is poor in chronic heart failure patients. J Cardiol Fail 2015;pii:S1071-9164(14)01369-4.

87. He FJ, MacGregor GA. Effect of longer-term modest salt reduction on blood pressure. Cochrane Database Syst Rev 2004;(3):CD004937.

88. The Trials of Hypertension Prevention Collaborative Research Group. Effects of weight loss and sodium reduction intervention on blood pressure and hypertension incidence in overweight people with high-normal blood pressure: the Trials of Hypertension Prevention, phase II. Arch Intern Med 1997;157:657-67.

89. Jula AM, Karanko HM. Effects on left ventricular hypertrophy of longterm nonpharmacological treatment with sodium restriction in mild-tomoderate essential hypertension. Circulation 1994;89:1023-31.

90. Cook NR, Cutler JA, Obarzanek E, et al. Long term effects of dietary sodium reduction on cardiovascular disease outcomes: observational follow-up of the Trials of Hypertension Prevention (TOHP). BMJ 2007;334:885-8.

91. Strazzullo P, D'Elia L, Kandala NB, et al. Salt intake, stroke, and cardiovascular disease: meta-analysis of prospective studies. BMJ 2009;339:b4567.

92. Gupta D, Georgiopoulou VV, Kalogeropoulos AP, et al. Dietary sodium intake in heart failure. Circulation 2012;126:479-85.

93. Paterna S, Parrinello G, Cannizzaro S, et al. Medium term effects of different dosage of diuretic, sodium, and fluid administration on neurohormonal and clinical outcome in patients with recently compensated heart failure. Am J Cardiol 2009;103:93-102.

94. Kenchaiah S, Evans JC, Levy D, et al. Obesity and the risk of heart failure. N Engl J Med 2002;347:305-13.

95. Habbu A, Lakkis NM, Dokainish H. The obesity paradox: fact or fiction? Am J Cardiol 2006;98:944-8.

96. MacDonald M, Fang J, Pittman SD, et al. The current prevalence of sleep disordered breathing in congestive heart failure patients treated with beta-blockers. J Clin Sleep Med 2008;4:38-42.

97. Bradley TD, Logan AG, Kimoff RJ, et al. Continuous positive airway pressure for central sleep apnea and heart failure. N Engl J Med 2005;353:2025-33.

98. Kaneko Y, Floras JS, Usui K, et al. Cardiovascular effects of continuous positive airway pressure in patients with heart failure and obstructive sleep apnea. N Engl J Med 2003;348:1233-41.

99. Mansfield DR, Gollogly NC, Kaye DM, et al. Controlled trial of continuous positive airway pressure in obstructive sleep apnea and heart failure. Am J Respir Crit Care Med 2004;169:361-6.

100. Huerta C, Varas-Lorenzo C, Castellsague J, et al. Nonsteroidal anti-inflammatory drugs and risk of first hospital admission for heart failure in the general population. Heart 2006;92:1610-5.
101. Gislason GH, Rasmussen JN, Abildstrom SZ, et al. Increased mortality and cardiovascular morbidity associated with use of nonsteroidal anti-inflammatory drugs in chronic heart failure. Arch Intern Med 2009;169:141-9.

102. Bouwe PK, Heeringa J, Hofman A, et al. Non steroidal anti-inflammatory drugs and the risk of atrial fibrillation: a population-based follow-up study. BMJ Open 2014;4:e004059.

103. Jackevicius CA, Fan CS, Warner AJ. Clinical outcomes of erythropoietin use in heart failure patients with anemia of chronic kidney disease. Cardiol Fail 2014;20: 327-33.

104. Stewart S, Jenkins A, Buchan S, et al. The current cost of heart failure to the National Health Service in the UK. Eur J Heart Fail 2002;4:361-71.

105. [No authors listed]. Effects of enalapril on mortality in severe congestive heart failure. Results of the Cooperative North Scandinavian Enalapril Survival Study (CONSENSUS). The CONSENSUS Trial Study Group. N Engl J Med 1987;316:1429-35.

106. [No authors listed]. Effect of enalapril on survival in patients with reduced left ventricular ejection fractions and congestive heart failure. The SOLVD Investigators. N Engl J Med 1991;325:293-302.

107. Garg R, Yusuf S. Overview of randomized trials of angiotensin-converting enzyme inhibitors on mortality and morbidity in patients with heart failure. Collaborative Group on ACE Inhibitor Trials. JAMA 1995;273:1450-6.

108. Packer M, Poole-Wilson PA, Armstrong PW, et al. Comparative effects of low and high doses of the angiotensin-converting enzyme inhibitor, lisinopril, on morbidity and mortality in chronic heart failure. ATLAS Study Group. Circulation 1999;100:2312-8.

109. The SOLVD Investigators. Effect of enalapril on mortality and the development of heart failure in asymptomatic patients with reduced left ventricular ejection fractions. N Engl J Med 1992;327:685-91.

110. [No authors listed]. The Cardiac Insufficiency Bisoprolol Study II (CIBIS-II): a randomised trial. Lancet 1999;353:9-13.

111. [No authors listed]. Effect of metoprolol CR/XL in chronic heart failure: Metoprolol CR/XL Randomised Intervention Trial in Congestive Heart Failure (MERITHF). Lancet 1999;353:2001-7.

112. Hjalmarson A, Goldstein S, Fagerberg B, et al. Effects of controlled-release metoprolol on total mortality, hospitalizations, and well-being in patients with heart failure: the Metoprolol CR/XL Randomized Intervention Trial in congestive heart failure (MERIT-HF). MERITHF Study Group. JAMA 2000;283:1295-302.

113. Packer M, Coats AJ, Fowler MB, et al. Effect of carvedilol on survival in severe chronic heart failure. $\mathrm{N}$ Engl J Med 2001;344:1651-8.

114. Packer M, Fowler MB, Roecker EB, et al. Effect of carvedilol on the morbidity of patients with severe chronic heart failure: results of the carvedilol prospective randomized cumulative survival (COPERNICUS) study. Circulation 2002;106:2194-9.

115. Flather MD, Shibata MC, Coats AJ, et al. Randomized trial to determine the effect of nebivolol on mortality and cardiovascular hospital admission in elderly patients with heart failure (SENIORS). Eur Heart J 2005; 26:215-25

116. Packer M, Bristow MR, Cohn JN, et al. The effect of 
carvedilol on morbidity and mortality in patients with chronic heart failure. U.S. Carvedilol Heart Failure Study Group. N Engl J Med 1996;334:1349-55.

117. Pitt B, Zannad F, Remme WJ, et al. The effect of spironolactone on morbidity and mortality in patients with severe heart failure. Randomized Aldactone Evaluation Study Investigators. N Engl J Med 1999;341:709-17.

118. Zannad F, McMurray JJV, Krum H, et al. Eplerenone in patients with systolic heart failure and mild symptoms. N Engl J Med 2011;364:11-21.

119. Fisher ML, Gottlieb SS, Plotnick GD, et al. Beneficial effects of metoprolol in heart failure associated with coronary artery disease: a randomized trial. J Am Coll Cardiol 1994;23:943-50.

120. Metra M, Nardi M, Giubbini R, et al. Effects of shortand long-term carvedilol administration on rest and exercise hemodynamic variables, exercise capacity and clinical conditions in patients with idiopathic dilated cardiomyopathy. J Am Coll Cardiol 1994;24:1678-87.

121. Olsen SL, Gilbert EM, Renlund DG, et al. Carvedilol improves left ventricular function and symptoms in chronic heart failure: a double-blind randomized study. J Am Coll Cardiol 1995;25:1225-31.

122. Krum H, Sackner-Bernstein JD, Goldsmith RL, et al. Double-blind, placebo-controlled study of the longterm efficacy of carvedilol in patients with severe chronic heart failure. Circulation 1995;92:1499-506.

123. Waagstein F, Bristow MR, Swedberg K, et al. Beneficial effects of metoprolol in idiopathic dilated cardiomyopathy: Metoprolol in Dilated Cardiomyopathy (MDC) Trial Study Group. Lancet 1993;342:1441-6.

124. Packer M, Colucci WS, Sackner-Bernstein JD, et al. Double-blind, placebocontrolled study of the effects of carvedilol in patients with moderate to severe heart failure: the PRECISE Trial: Prospective Randomized Evaluation of Carvedilol on Symptoms and Exercise. Circulation 1996;94:2793-9.

125. Colucci WS, Packer M, Bristow MR, et al. Carvedilol inhibits clinical progression in patients with mild symptoms of heart failure: US Carvedilol Heart Failure Study Group. Circulation 1996;94:2800-6.

126. CIBIS II Authors. The Cardiac Insufficiency Bisoprolol Study II (CIBIS-II): a randomised trial. Lancet 1999; 353:9-13.

127. Packer M, Bristow MR, Cohn JN, et al. The effect of carvedilol on morbidity and mortality in patients with chronic heart failure: U.S. Carvedilol Heart Failure Study Group. N Engl J Med 1996;334:1349-55.

128. Australia/New Zealand Heart Failure Research Collaborative Group. Randomised, placebo-controlled trial of carvedilol in patients with congestive heart failure due to ischaemic heart disease. Lancet 1997;349:375-80.

129. Ali A, Fonarow G, Sanders C, et al. Renin-angiotensin inhibition in systolic heart failure and chronic kidney disease. Am J Med 2012;125:399-410.

130. Maggioni AP, Anand I, Gottlieb SO, et al. Effects of valsartan on morbidity and mortality in patients with heart failure not receiving angiotensin-converting enzyme inhibitors. J Am Coll Cardiol 2002;40:1414-21.

131. Zannad F, McMurray JJV, Krum H, et al. Eplerenone in patients with systolic heart failure and mild symptoms. N Engl J Med 2011;364:11-21.

132. Pitt B, Zannad F, Remme WJ, et al. Randomized Aldac- tone Evaluation Study Investigators. The effect of spironolactone on morbidity and mortality in patients with severe heart failure. N Engl J Med 1999;341:709-17.

133. Swedberg K, Komajda M, Bohm M, et al. Ivabradine and outcomes in chronic heart failure (SHIFT): a randomised placebo-controlled study. Lancet 2010;376: 875-85.

134. The effect of digoxin on mortality and morbidity in patients with heart failure. The Digitalis Investigation Group. N Engl J Med 1997;336:525-33.

135. The Captopril-Digoxin Multicenter Research Group. Comparative effects of therapy with captopril and digoxin in patients with mild to moderate heart failure. JAMA 1988;259:539-44.

136. Dobbs SM, Kenyon WI, Dobbs RJ. Maintenance digoxin after an episode of heart failure: placebo-controlled trial in outpatients. Br Med J 1977;1:749-52.

137. Lee DC, Johnson RA, Bingham JB, et al. Heart failure in outpatients: a randomized trial of digoxin versus placebo. N Engl J Med 1982;306:699-705.

138. Guyatt GH, Sullivan MJ, Fallen EL, et al. A controlled trial of digoxin in congestive heart failure. Am J Cardiol 1988;61:371-5.

139. DiBianco R, Shabetai R, Kostuk W, et al. A comparison of oral milrinone, digoxin, and their combination in the treatment of patients with chronic heart failure. N Engl J Med 1989;320:677-83.

140. Uretsky BF, Young JB, Shahidi FE, et al. Randomized study assessing the effect of digoxin withdrawal in patients with mild to moderate chronic congestive heart failure: results of the PROVED trial: PROVED Investigative Group. J Am Coll Cardiol 1993;22:955-62.

141. Packer M, Gheorghiade M, Young JB, et al. Withdrawal of digoxin from patients with chronic heart failure treated with angiotensin-convertingenzyme inhibitors: RADIANCE Study. N Engl J Med 1993;329:1-7.

142. Cohn JN, Archibald DG, Ziesche S, et al. Effect of vasodilator therapy on mortality in chronic congestive heart failure. Results of a Veterans Administration Cooperative Study. N Engl J Med 1986;314:1547-52.

143. Cohn JN, Johnson G, Ziesche S, et al. A comparison of enalapril with hydralazine-isosorbide dinitrate in the treatment of chronic congestive heart failure. N Engl J Med 1991;325:303-10.

144. Taylor AL, Ziesche S, Yancy C, et al. Combination of isosorbide dinitrate and hydralazine in blacks with heart failure. N Engl J Med 2004;351:2049-57.

145. Tavazzi L, Maggioni AP, Marchioli R, et al. Effect of $\mathrm{n}-3$ polyunsaturated fatty acids in patients with chronic heart failure (the GISSI-HF trial): a randomised, doubleblind, placebo-controlled trial. Lancet 2008;372: 1223-30.

146. [No authors listed]. Effect of verapamil on mortality and major events after acute myocardial infarction (the Danish Verapamil Infarction Trial II-DAVIT II). Am J Cardiol 1990;66:779-85.

147. Goldstein RE, Boccuzzi SJ, Cruess D, et al. Diltiazem increases late-onset congestive heart failure in postinfarction patients with early reduction in ejection fraction: the Adverse Experience Committee; and the Multicenter Diltiazem Postinfarction Research Group. Circulation 1991;83:52-60.

148. The Multicenter Diltiazem Postinfarction Trial Research Group. The effect of diltiazem on mortality and 
reinfarction after myocardial infarction. N Engl J Med 1988;319:385-92.

149. Figulla HR, Gietzen F, Zeymer U, et al. Diltiazem improves cardiac function and exercise capacity in patients with idiopathic dilated cardiomyopathy: results of the Diltiazem in Dilated Cardiomyopathy Trial. Circulation 1996;94:346-52.

150. Elkayam U, Amin J, Mehra A, et al. A prospective, randomized, doubleblind, crossover study to compare the efficacy and safety of chronic nifedipine therapy with that of isosorbide dinitrate and their combination in the treatment of chronic congestive heart failure. Circulation 1990;82:1954-61.

151. Packer M, O'Connor CM, Ghali JK, et al. Effect of amlodipine on morbidity and mortality in severe chronic heart failure: Prospective Randomized Amlodipine Survival Evaluation Study Group. N Engl J Med 1996;335: 1107-14

152. Cohn JN, Ziesche S, Smith R, et al. Effect of the calcium antagonist felodipine as supplementary vasodilator therapy in patients with chronic heart failure treated with enalapril: V-HeFT III. Vasodilator-Heart Failure Trial (V-HeFT) Study Group. Circulation 1997;96:856-63.

153. Littler WA, Sheridan DJ. Placebo controlled trial of felodipine in patients with mild to moderate heart failure: UK Study Group. Br Heart J 1995;73:428-33.

154. Udelson JE, DeAbate CA, Berk M, et al. Effects of amlodipine on exercise tolerance, quality of life, and left ventricular function in patients with heart failure from left ventricular systolic dysfunction. Am Heart J 2000;139:503-10.

155. Thackray S, Witte K, Clark AL, et al. Clinical trials update: OPTIMECHF, PRAISE-2, ALL-HAT. Eur J Heart Fail 2000;2:209-12.

156. Mahé I, Chassany O, Grenard AS, et al. Defining the role of calcium channel antagonists in heart failure due to systolic dysfunction. Am J Cardiovasc Drugs 2003;3:33-41.

157. Waldo AL, Camm AJ, deRuyter H, et al. Effect of dsotalol on mortality in patients with left ventricular dysfunction after recent and remote myocardial infarction: the SWORD Investigators: Survival With Oral d-Sotalol. Lancet 1996;348:7-12.

158. Kober L, Torp-Pedersen C, McMurray JJ, et al. Increased mortality after dronedarone therapy for severe heart failure. N Engl J Med 2008;358:2678-87.

159. The Cardiac Arrhythmia Suppression Trial (CAST) Investigators. Preliminary report: effect of encainide and flecainide on mortality in a randomized trial of arrhythmia suppression after myocardial infarction. N Engl J Med 1989;321:406-12.

160. Doval HC, Nul DR, Grancelli HO, et al. Randomised trial of low-dose amiodarone in severe congestive heart failure: Grupo de Estudio de la Sobrevida en la Insuficiencia Cardiaca en Argentina (GESICA). Lancet 1994;344:493-8.

161. Singh SN, Fletcher RD, Fisher SG, et al. Amiodarone in patients with congestive heart failure and asymptomatic ventricular arrhythmia: Survival Trial of Antiarrhythmic Therapy in Congestive Heart Failure. N Engl J Med 1995;333:77-82.

162. Torp-Pedersen C, Moller M, Bloch-Thomsen PE, et al.
Dofetilide in patients with congestive heart failure and left ventricular dysfunction: Danish Investigations of Arrhythmia and Mortality on Dofetilide Study Group. N Engl J Med 1999;341:857-65.

163. Kober L, Bloch Thomsen PE, Moller M, et al. Effect of dofetilide in patients with recent myocardial infarction and left-ventricular dysfunction: a randomised trial. Lancet 2000;356:2052-8.

164. Fuster V, Gersh BJ, Giuliani ER, et al. The natural history of idiopathic dilated cardiomyopathy. Am J Cardiol 1981;47:525-31.

165. Stratton JR, Nemanich JW, Johannessen KA, et al. Fate of left ventricular thrombi in patients with remote myocardial infarction or idiopathic cardiomyopathy. Circulation 1988;78:1388-93.

166. Jafri SM. Hypercoagulability in heart failure. Semin Thromb Hemost 1997;23:543-5.

167. Homma S, Thompson JL, Pullicino PM, et al. Warfarin and aspirin in patients with heart failure and sinus rhythm. N Engl J Med 2012;366:1859-69.

168. Bermingham M, Shanahan MK, O’Connell E, et al. Aspirin use in heart failure: is low-dose therapy associated with mortality and morbidity benefits in a large community population? Circ Heart Fail 2014; 7:243-50.

169. Reiner Z, Catapano AL, De Backer G, et al. ESC/EAS Guidelines for the management of dyslipidaemias: the Task Force for the management of dyslipidaemias of the European Society of Cardiology (ESC) and the European Atherosclerosis Society (EAS). Eur Heart J 2011;32:1769-818.

170. Kjekshus J, Apetrei E, Barrios V, et al. Rosuvastatin in older patients with systolic heart failure. N Engl J Med 2007;357:2248-61.

171. Jessup M, Fox KAA, Komajda M, et al. PARADIGMHF - The Experts' Discussion. N Engl J Med 2014; 371:e15.

172. McMurray JJV, Packer M, Desai AS, et al. Neprilysin inhibition versus enalapril in heart failure. N Engl J Med 2014;371:993-1004.

173. Setaro JF, Zaret BL, Schulman DS, Black HR, Soufer $R$. Usefulness of verapamil for congestive heart failure associated with abnormal left ventricular diastolic filling and normal left ventricular systolic performance. Am J Cardiol 1990;66:981-6.

174. Hung MJ, Cherng WJ, Kuo LT, et al. Effect of verapamil in elderly patients with left ventricular diastolic dysfunction as a cause of congestive heart failure. Int $\mathrm{J}$ Clin Pract 2002;56:57-62.

175. Broder H, Gottlieb RA, Lepor NE. Chemotherapy and cardiotoxicity. Rev Cardiovasc Med 2008;9:75-83.

176. Anker SD, Comin Colet J, Filippatos G, et al. Ferric carboxymaltose in patients with heart failure and iron deficiency. N Engl J Med 2009;361:2436-48.

177. McDonagh T, Macdougall IC. Iron therapy for the treatment of iron deficiency in chronic heart failure: intravenous or oral? Eur J Heart Fail 2015;17:248-62.

178. McMurray JJV, Holman RR, Haffner SM, et al. The NAVIGATOR Study Group. Effect of valsartan on the incidence of diabetes and cardiovascular events. N Engl J Med 2010;362:1477-90.

179. Bakris GL, Fonseca V, Katholi RE, et al. for the GEMINI Investigators. Metabolic Effects of carvedilol vs metoprolol in patients with type 42 diabetes mellitus 
and hypertension-a randomized contolled trial. JAMA 2004;292:2227-36.

180. Komajda M, McMurray JJV, Beck-Nielsen H, et al. Heart failure events with rosiglitazone in type 2 diabetes: data from the RECORD clinical trial. Eur Heart J 2010;31:824-31.

181. Hernandez AV, Usmani A, Rajamanickam A, et al. Thiazolidinediones and risk of heart failure in patients with or at high risk of type 2 diabetes mellitus: a meta-analysis and meta-regression analysis of placebo-controlled randomized clinical trials. Am J Cardiovasc Drugs 2011;11:115-28.

182. Erdmann E, Charbonnel B, Wilcox RG, et al. Pioglitazone use and heart failure in patients with type 2 diabetes and preexisting cardiovascular disease: data from the PROactive study (PROactive 08). Diabetes Care 2007;30:2773-8.

183. MacDonald MR, Eurich DT, Majumdar SR, et al. Treatment of type 2 diabetes and outcomes in patients with heart failure: a nested case-control study from the U.K. General Practice Research Database. Diabetes Care 2010;33:1213-8.

184. Warriner DR, Sheridan PJ. End-stage heart failure nonpharmacological therapy: recent advances in pacemakers, pressure monitors, pumps and other devices. Postgrad Med J 2014;90:164-70.

185. [No authors listed]. A comparison of antiarrhythmicdrug therapy with implantable defibrillators in patients resuscitated from near-fatal ventricular arrhythmias. The Antiarrhythmics versus Implantable Defibrillators (AVID) Investigators. N Engl J Med 1997;337:1576-83.

186. Oseroff O, Retyk E, Bochoeyer A. Subanalyses of secondary prevention implantable cardioverter-defibrillator trials: antiarrhythmics versus implantable defibrillators (AVID), Canadian Implantable Defibrillator Study (CIDS), and Cardiac Arrest Study Hamburg (CASH). Curr Opin Cardiol 2004;19:26-30.

187. Bardy GH, Lee KL, Mark DB, et al. Amiodarone or an implantable cardioverter-defibrillator for congestive heart failure. N Engl J Med 2005;352:225-37.

188. Moss AJ, Hall WJ, Cannom DS, et al. Cardiac resynchronization therapy for the prevention of heart-failure events. N Engl J Med 2009;361:1329-38.

189. Tang AS, Wells GA, Talajic M, et al. Cardiac-resynchronization therapy for mild-to-moderate heart failure. N Engl J Med 2010;363:2385-95.

190. Bristow MR, Saxon LA, Boehmer J, et al. Cardiac-resynchronization therapy with or without an implantable defibrillator in advanced chronic heart failure. $\mathrm{N}$ Engl J Med 2004;350:2140-50.

191. Cleland JG, Daubert JC, Erdmann E, et al. The effect of cardiac resynchronization on morbidity and mortality in heart failure. N Engl J Med 2005;352:1539-49.

192. Wijns W, Kolh P, Danchin N, et al. Guidelines on myocardial revascularization. Eur Heart J 2010;31:2501-55.

193. Vahanian A, Alfieri O, Andreotti F, et al. Guidelines on the Management of Valvular Heart Disease (Version 2012). The Joint Task Force on the Management of Valvular Heart Disease of the European Society of Cardiology (ESC) and the European Association for Cardio-Thoracic Surgery (EACTS). Eur Heart J 2012; 33:2451-96

194. Banner NR, Bonser RS, Clark AL, et al. UK guidelines for referral and assessment of adults for heart transplantation. Heart 2011;97:1520-7.

195. Mehra MR, Kobashigawa J, Starling R, et al. Listing criteria for heart transplantation: International Society for Heart and Lung Transplantation guidelines for the care of cardiac transplant candidates - 2006. J Heart Lung Transplant 2006;25:1024-42.

196. Dharmarajan K, Hsieh AF, Lin Z, et al. Diagnosis and timing of 30-Day readmission after hospitalization of heart failure, acute myocardial infarction or pneumonia. JAMA 2013;309:355-63.

197. Aranda JM Jr, Johnson JW, Conti JB. Current trends in heart failure readmission rates analysis of Medicare data. Cin Cardiol 2009;32:47-52.

198. Dharmarajan K, Hsieh AF, Kulkarni VT, et al. Trajectories of risk after hospedalization for HF, IMA or pneumonia :retrospective cohort study. BMJ 2015;350:h411.

199. Philbin EF, Dech GW, Jenkins PL, et al. Socioeconomic status as an independent risk factor for hospital readmission in human failure. Am J Cardiol 2001;87:1367-71.

200. Ross JS, Mulvey GK, Stauffer B, et al. Statistical models and patient predictors of readmission for heart failure: a systematic review. Arch Intern Med 2008;168: 1371-86.

201. Gonseth J, Guallar-Carillon P, Banegas JR, et al. The effectiveness of disease management programmes in reducing hospital readmission in older patients with Human Failure: a systematic review and meta-analisys published reports. Eur Heart J 2004;25:1570-92.

202. National Institute for Health and Care Excellence (NICE). Management of chronic heart failure in adults in primary and secondary care. NICE guidelines [CG108]; August 2010. Available from: http://www.nice. org.uk/guidance/cg108

203. Mant J, Al-Mohammad A, Swain S, et al. Management of chronic heart failure in adults: synopsis excellence guideline. Ann Int Med 2011;155:252-9.

204. Small RS, Wickemeyer W, Germany R, et al. Changes in intrathoracic impedance are associated with subsequent risk of hospitalizations for acute decompensated heart failure: clinical utility of implanted device monitoring without a patient alert. J Cardiol Fail 2009; $15: 475-81$.

205. Perego GB, Landolina M, Vergara G, et al. Implantable CRT device diagnostics identify patients with increased risk for heart failure hospitalization. J Int Card Electrophysiol 2008;23:235-42.

206. Zile MR, Bourge RC, Bennett TD, et al. Application of implantable haemodynamic monitoring in the management of patients with diastolic heart failure: a subgroup analysis of the COMPASS-HF trial. J Card Fail 2008; 14:816-23.

207. Tang WHW, Francis GS, MD. The year in heart failure. J Am Coll Cardiol 2010;55:688-96.

208. Tsai TC, Joynt KE, Orav RJ, et al. Variation in surgical readmission and quality of hospital care. New Engl J Med 2013;369:1134-42.

209. Di Lenarda A. Consensus Conference. Il percorso assistenziale del paziente con scompenso cardiaco. G Ital Cardiol 2006;7:387-432.

210. Lee DS, Tran C, Flintoft V, et al. CCORT/CCS quality indicators for congestive heart failure care. Can J Cardiol 2003;19 357-64. 
211. Bonow RO, Bennett S, Casey DE Jr, et al. ACC/AHA clinical performance measures for adults with chronic heart failure: a report of the American College of Cardiology/American Heart Association Task Force on Performance Measures (Writing Committee to Develop Heart Failure Clinical Performance Measures) endorsed by the Heart Failure Society of America. J Am Coll Cardiol 2005;46:1144-78.

212. Krumholz HM, Baker DW, Ashton CM, et al. CHF QUERI. Quality enhancement research initiative. Evaluating quality of care for patients with heart failure. Cir- culation 2000;101:e122-40.

213. Gibbons RJ, Smith C, Antman R, et al. American College of Cardiology/American Heart Association clinical practice guidelines: Part II: Evolutionary changes in a continuous quality improvement project. Circulation 2003;107:3101-7.

214. Faxon DP, Schwamm LH, Pasternak RC, et al. Improving quality of care through disease management: principles and recommendations from the American Heart Association's Expert Panel on Disease Management. Circulation 2004;109:2651-64. 


\title{
Prevenzione secondaria degli eventi cerebrovascolari aterotrombotici
}

\author{
Maurizio Paciaroni, ${ }^{1}$ Roberto Frediani, ${ }^{2}$ Michele Stornello, ${ }^{3}$ Giancarlo Agnelli ${ }^{1}$ \\ ${ }^{1}$ Medicina Interna e Stroke Unit, Università degli studi di Perugia, Ospedale Santa Maria della Misericordia, Perugia; ${ }^{2}$ ASL \\ VCO - Ospedali di Verbania e Domodossola; ${ }^{3}$ UO Medicina Interna \& Stroke Unit, Centro per lo studio e la cura della iperten- \\ sione arteriosa e la prevenzione delle cardio e cerebrovasculopatie L. Scapellato, Ospedale Umberto I, Siracusa, Italia
}

\section{Introduzione}

L'ictus è la seconda causa di morte nel mondo (terza negli USA) e la principale causa di invalidità. Ciò si realizza con circa 500 attacchi ischemici e 2400 nuovi ictus per milione di abitanti/anno. Un terzo dei nuovi ictus muore ogni anno mentre poco meno della metà perde la propria indipendenza. ${ }^{1} \mathrm{La}$ prevenzione primaria e secondaria rappresentano un punto strategico fondamentale per evitare l'ictus e ridurne significativamente la ricorrenza. In questa review tratteremo della prevenzione secondaria dell'ictus.

\section{Prevenzione secondaria precoce}

\section{Attacchi ischemici transitori}

L'attacco ischemico transitorio (TIA) è associato ad un alto rischio di ictus ricorrente e precoce con una frequenza che può raggiungere valori significativi dalle prime 24 ore e sino al trentesimo giorno. ${ }^{2-4} \mathrm{La}$ prevenzione secondaria dopo un TIA deve quindi essere iniziata subito. Lo score $\mathrm{ABCD}^{2}$ è stato sviluppato per migliorare la stratificazione precoce del rischio di ictus dopo un TIA e viene anche usato come riferimento nelle linee guida internazionali; la tomografia assiale computerizzata e la risonanza magnetica possono anche dare informazioni prognostiche. ${ }^{2,5-12}$ Gli studi EXPRESS e SOS-TIA dimostrano che una prognosi favorevole si può avere con l'inizio precoce ed intensivo del trattamento preventivo. ${ }^{13,14}$

Corrispondente: Michele Stornello, UO Medicina Interna \& Stroke Unit, Ospedale Umberto I, via Testaferrata 1, 96100 Siracusa, Italia.

E-mail: m.stornello@asp.sr.it

Articolo pubblicato secondo la Creative Commons Attribution NonCommercial 3.0 License (CC BY-NC 3.0).

(C) Copyright M. Paciaroni et al., 2015

Licensee PAGEPress, Italy

QUADERNI - Italian Journal of Medicine 2015; 3:356-363

\section{Ictus ichemico aterotrombotico}

La ricorrenza di ictus ischemico causata dalla aterosclerosi è più frequente di quella sostenuta da altri tipi di ictus ${ }^{15}$ e ciò è stato dimostrato in studi che hanno compreso un periodo che va dal settimo giorno ai tre mesi dall'episodio acuto e per varie cause di ictus. ${ }^{16}$ L'inizio entro 48 ore dalla comparsa dell'ictus della prevenzione secondaria con aspirina (160-300 $\mathrm{mg})$ ha ridotto la mortalità e la morbidità verosimilmente riducendo la recidiva dell'ictus. ${ }^{17} \mathrm{Vi}$ è più di una evidenza che dimostra la validità del trattamento antiaggregante intensivo nella fase acuta di un ictus minore o di un TIA. ${ }^{18-20} \mathrm{La}$ aspirina in associazione al coplidogrel si è rivelata efficace nel ridurre il rischio di recidiva di ictus anche nella stenosi sintomatica della carotide e dei vasi intracranici. ${ }^{21,22}$ La terapia anticoagulante non ha mostrato benefici nella fase acuta dell'ictus. ${ }^{23} \mathrm{La}$ endarteriectomia carotidea nella stenosi severa della carotide si è mostrata efficace quando effettuata entro due settimane dall'episodio acuto ictale non disabilitante e/o dal $\mathrm{TIA}^{24}$ con qualche incertezza se effettuato entro le prime 48 ore. $^{25}$

\section{Prevenzione secondaria cronica}

\section{Controllo severo di tutti i fattori di rischio}

Vi sono varie strategie per prevenire la recidiva di ictus o TIA secondari ad aterosclerosi. Sebbene non vi siano dati che dimostrino la efficacia della cessazione del fumo, della astinenza dall'alcool, del buon controllo glicemico e del peso corporeo, dell'attività fisica, tuttavia non può non essere evidente che questi fattori possono giocare un ruolo rilevante nella prevenzione di recidive di ictus. Può apparire interessante ma non conclusivo che supplementi di vitamine B6 e B12 possano ridurre il rischio di recidive ictali anche se i dati non raggiungono livelli di adeguata significatività. ${ }^{26}$

\section{Ipertensione arteriosa}

L'ipertensione e l'età sono I più potenti fattori di rischio per l'ictus. ${ }^{27}$ L'incidenza di ipertensione nel 
mondo è in crescita sia nei paesi economicamente sviluppati che in quelli in via di sviluppo e si calcola che nel 2025 un terzo della popolazione mondiale sarà affetto da ipertensione arteriosa ${ }^{28,29}$ La riduzione della pressione arteriosa riduce significativamente il rischio del primo ictus e di recidiva dello stesso ma rimane non dimostrato il vantaggio protettivo specifico di un antipertensivo su di un altro. ${ }^{30}$ Pochi sono $i$ trials effettuati con antipertensivi nella prevenzione secondaria dello stroke con risultati differenti dovuti alla diversità dei farmaci valutati. ${ }^{31-35}$ Una metanalisi ha riportato che la terapia antipertensiva è risultata efficace nel ridurre le recidive di ictus (odds ratio 0,76 ), infarto del miocardio (odds ratio 0,79 ) ed eventi vascolari (odds ratio 0,79). ${ }^{36}$ Tuttavia la eterogeneità tra $i$ farmaci è risultata evidente: i) i $\beta$ bloccanti non sembrano ridurre eventi vascolari; ii) i diuretici da soli riducono il rischio di ictus ma non di infarto miocardico; iii) gli ACE-inibitori riducono l'infarto del miocardio ma non lo stroke; iv) la combinazione di ACE-inibitore e diuretico si è rivelata essere il trattamento più efficace con una significativa riduzione di tutti e tre gli outcomes. In questa review sistematica non erano inclusi i sartani poichè i dati sulla prevenzione secondaria dell'ictus con questa classe di farmaci sono pochi; v) i calcioantagonisti, che con i diuretici riducono la variabilità pressoria, indice ormai riconosciuto di aumentato rischio cardiovascolare, sono più efficaci degli inibitori del RAS e soprattutto dei betabloccanti nella prevenzione primaria e scondaria dello stroke; ${ }^{37-39}$ solo in uno studio il bloccante i recettori per l'angiotensina ha mostrato una maggiore efficacia del calcio antagonista nella prevenzione di recidiva di TIA o ictus a parità di efficacia antiipertensiva. ${ }^{34}$

In conclusione la riduzione della pressione arteriosa è un metodo di per sè efficace nel limitare il rischio di recidiva di ictus, e questo vale anche per l'ipertensione lieve moderata. ${ }^{40}$ La maggior parte dei pazienti necessita di due o più farmaci logicamente combinati, tenendo anche conto degli effetti di questi sul sistema renina-angiotensina. ${ }^{41,42}$ Rimane la vexata quaestio di quanto sia necessario abbassare la pressione per quanto riguarda l'ictus: se nella fase cronica dello stroke non è affatto dimostrata una curva $\mathrm{J}$, nella fase acuta il discorso è diverso: uno studio recente ha dimostrato che una bassa pressione arteriosa media $(<100 \mathrm{mmHg})$ al momento del ricovero ed una pressione sistolica inferiore a 120 $\mathrm{mmHg}$ al momento della dimissione aumentano la mortalità totale. ${ }^{43}$

\section{Pressione arteriosa e recidiva di ictus in pazienti con stenosi intracraniche}

In contrasto con gli effetti benefici della riduzione della pressione arteriosa nella riduzione del rischio di recidiva di ictus, in pazienti con stenosi intracraniche sembra essere pratica comune mantenere la pressione $\geq 140 / 90 \mathrm{mmHg}$. Questa pratica sarebbe suggerita dalla opinione di esperti e da alcuni studi che suggerirebbero che la riduzione della pressione arteriosa in alcuni pazienti con stenosi severa della carotide aumenterebbe il rischio di ictus. ${ }^{44}$ I risultati del trial warfarin-aspirin symptomatic intracranial disease (WASID) concludono invece contro la pratica del mantenimento di alti valori pressori dimostrando anche in questi pazienti la validità della riduzione della pressione nella significativa diminuzione del rischio di recidiva ictale. ${ }^{45}$

\section{Uso di estrogeni}

La terapia sostitutiva con estrogeni nelle donne in post menopausa non si è dimostrata efficace nè in prevenzione primaria nè in secondaria. ${ }^{46}$ In uno studio l'uso di estrogeni ha dimostrato un trend peggiorativo ma non significativo verso la recidiva di ictus.

\section{Metabolismo dei lipidi}

Le dislipidemie sono tra i fattori di rischio delle malattie vascolari. Se la relazione tra dislipidemie e coronaropatie è ormai un dato acquisito, quella con l'ictus rimane controversa. Infatti se da una parte una metanalisi condotta su 45 studi prospettici non ha evidenziato alcuna relazione tra colesteroloemia ed ictus $^{47}$ un'altra metanalisi di studi prospettici ha trovato un rischio relativo di ictus pari a 1,2 tra quelli con un colesterolo totale più alto di $220 \mathrm{mg} / \mathrm{dL}{ }^{48}$ Altri studi hanno dimostrato un aumento del rischio di ictus non emorragico correlato a livelli più o meno elevate di colesterolo. ${ }^{49,50}$ Può esserci più di una spiegazione per spiegare questi differenti risultati e per la apparente mancanza di relazione tra i livelli di colesterolo e l'ictus. La maggior parte degli studi sono stati indirizzati ad osservare la relazione tra colesterolo e coronaropatie, pazienti più giovani ed a più alto rischio di infarto piuttosto che di ictus. Per di più non sono stati analizzati i sottotipi di ictus (emorragico o ischemico). Il multiple risk factor intervention trial (MRFIT) mostra che il rischio di morte dovuto ad ictus aumenta con l'aumentare dei livelli di colesterolo e, nello stesso studio, il rischio di ictus emorragico è inversamente correlato ai livelli di colesterolo. Ciò suggerirebbe la presenza di una sorta di curva J. Se realmente presente e confermata questa curva $\mathrm{J}$ potrebbe aver mascherato la diminuzione di ictus ischemici non facendo così rilevare la relazione tra incremento di colesterolo ed ictus, avendo contato gli ictus emorragici insieme agli ischemici. ${ }^{51,52}$ Infine e purtroppo esistono pochi studi sulla correlazione tra colesterolemia e sottotipi di ictus. Le statine sono state ampiamente studiate in pazienti con coronaropatie ed infarto miocardico recente. Sono rilevanti i 
risultati in sottopopolazioni con TIA o ictus nell'ambito della prevenzione primaria. Comunque, dati recenti indicano che le statine si sono rilevate efficaci nel ridurre le recidive di ictus. Questo effetto non sembrerebbe essere dovuto esclusivamente la riduzione del colesterolo ma anche ad effetti pleiotropici non del tutto conosciuti. Tuttavia, dati recenti di letteratura indicano che le statine possono essere efficaci nel ridurre le recidive di stroke. ${ }^{53,54}$ Lo studio stroke prevention by aggressive reduction of cholesterol levels (SPARCL) è stato il primo a valutare gli effetti delle statine in pazienti senza storia di coronaropatia ma che avevano avuto un TIA o un ictus. $\mathrm{Fu}$ rilevata una riduzione del rischio di ictus associata anche ad una significativa diminuzione del rischio di malattia coronarica. ${ }^{55}$ In linea teorica l'uso delle statine potrebbe indure aumento del rischio di emorragia cerebrale ma ciò non è stato osservato in prevenzione primaria. ${ }^{56}$ Nello studio SPARCL, condotto come detto in prevenzione secondaria, vi fu un aumento di emorragie intracerebrali nei pazienti trattati con statina ma in termini di riduzione globale del rischio di ictus il trattamento si è rivelato benefico. Una analisi secondaria dello studio stesso condotta sui pazienti che avevano avuto la emorragia intracerebrale non ha mostrato una relazione tra questa e la riduzione del colesterolo con lipoproteine a bassa densità. ${ }^{57}$

Questi risultati mostrano che le statine hanno un profilo di sicurezza buono ma a dispetto di ciò necessitano ulteriori studi per escludere che statine giochino un ruolo nella comparsa di emorragie intracerebrali in prevenzione secondaria.

\section{Il trattamento della malattia carotidea}

\section{Endoarteriectomia carotidea}

La endoarteriectomia carotidea si è dimostrata efficace nel ridurre significativamente il rischio di ictus ischemico nei pazienti con stenosi delle carotidi sintomatiche. A questo proposito lo studio North American symptomatic carotid endarterectomy trial (NASCET) ha evidenziato che in pazienti con TIA o minor stroke e stenosi ipsilaterale del $70 \%$, la endoarteriectomia si è mostrata più efficace della terapia antiaggregante riducendo significativamente il rischio relativo ed assoluto di ictus. ${ }^{52}$ Nello stesso studio la correzione delle stenosi moderate (50-69\%) ha avuto effetti minori sulla riduzione del rischio, ciò anche in relazione ai concomitanti fattori di rischio. ${ }^{48} \mathrm{Il}$ Veterans Administration cooperative study ed il European carotid surgery trial (ECST) hanno confermato i benefici della endoarteriectomia nelle stenosi maggiori del $50 \%$, mentre nessun beneficio è stato dimostrato per interventi in stenosi inferiori. ${ }^{58}$ Sulla base di tutto ciò si è raggiunto un consenso nell'affermare che, in pazienti con TIA o minor stroke e con una stenosi del $70 \%$ o maggiore, la endoarteriectomia è la migliore opzione preventiva per le recidive di ictus. Di contro l'approccio chirurgico non ha benefici per le stenosi inferiori del $50 \%$ mentre per quelle tra il $50-69 \%$ e sintomi ipsilaterali gli outcomes dell'endoarteriectomia e quindi il suo consiglio, dipende dalla stratificazione del rischio di quel paziente. Si è infine osservato che i trattamenti entro le due settimane dall'episodio acuto hanno un significativo maggior effetto preventivo rispetto agli interventi tardivi e questo soprattutto nelle donne. ${ }^{59}$

\section{Angioplastica e stent}

$\mathrm{La}$ angioplastica ed il posizionamento di stent per il trattamento non chirurgico delle stenosi carotidee attraverso tecniche endovascolari hanno dato esiti controversi. ${ }^{60-63}$ Una metanalisi di tutti i dati disponibili anche senza quelli dello studio ICSS ${ }^{62}$ significativamente sfavorevoli alla procedura endovascolare) ha mostrato un significativo peggioramento degli esiti dopo il trattamento endovascolare. ${ }^{64,65}$

\section{Antiaggreganti}

Il ruolo delle piastrine nella patogenesi dell'aterotrombosi è ormai ben documentato e noto. ${ }^{66} \mathrm{La}$ efficacia della terapia antiaggregante per la prevenzione secondaria dell'ictus è stata dimostrata con più di un trial randomizzato e con metanalisi. L'aspirina, la ticlopidina, il dipiridamolo, il cilostazol ed il trifusal sono stati studiati per le loro peculiarità di antiaggreganti nella prevenzione secondaria dell'ictus.

\section{Aspirina \\ È soprattutto con la metanalisi dell'Antithrombo- tic Trialists' Collaboration che si è dimostrata la ef- ficacia della aspirina in pazienti con TIA o ictus nel ridurre significativamente le recidive senza diffe- renze di genere, di età ed a prescindere dalla presenza o meno di ipertensione e di diabete. Effetti favorevoli sono stati dimostrati anche per quello che riguarda l'infarto del miocardio e gli eventi vascolari. Le dosi raccomandate suggerite per la prevenzione seconda- ria dopo un TIA o un ictus sono di 50-325 mg di aspi- rina e ciò senza significativo aumento del rischio emorragico. ${ }^{67}$}

\section{Ticlopidina}

Il Canadian American ticlopidine study ed il ticlopidine aspirin stroke study, hanno dimostrato la efficacia di questo antiaggregante nella prevenzione secondaria che nel secondo è apparsa maggiore rispetto a quella della aspirina a dosi di $1300 \mathrm{mg} / \mathrm{die}$. Tutto ciò contrasta con i risultati dell' African American antiplatelet stroke study che ha fallito nel dimo- 
strare benefici rispetto alla aspirina in pazienti ad alto rischio. ${ }^{68}$ La ticlopidina è comunque raramente utilizzata a causa dei suoi severi effetti indesiderati tra $\mathrm{i}$ quali la neutropenia.

\section{Dipiridamolo}

Mentre lo European stroke prevention study ha dimostrato la pari efficacia in prevenzione secondaria di $200 \mathrm{mg}$ b.i.d. di dipiridamolo rispetto alla aspirina $25 \mathrm{mg}$ b.i.d. ed un maggiore efficacia della loro combinazione ${ }^{69}$ lo studio ESPRIT non ha confermato questi dati. ${ }^{70}$

\section{Clopidogrel}

Il trial clopidogrel versus aspirin in patients at risk of ischemic events (CAPRIE), $75 \mathrm{mg}$ di clopidogrel in confronto a $325 \mathrm{mg}$ di aspirina, ha mostrato una significativa efficacia di questo antiaggregante nella prevenzione di recidive. ${ }^{71}$

\section{Cilostazol}

Il cilostazol è un antiaggregante che inibisce la fosfodiesterasi 3 ed aumenta il cAMP. Nello studio condotto in Giappone, CSPS-2, in confronto alla aspirina si è mostrato efficace nella riduzione delle recidive di ictus e nelle complicanze emorragiche. ${ }^{72}$

\section{Triflusal}

Il triflusal è simile alla aspirina sia da un punto di vista strutturale sia per ciò che attiene alla sua efficacia per la prevenzione degli eventi vascolari. ${ }^{73}$

\section{Terapie di combinazione}

Il dipiridamolo associato alla aspirina si è dimostrato efficace nella prevenzione secondaria a lungo termine dell'ictus o morte. Il trial management of atherothrombosis with clopidogrel in high-risk patients with recent TIA or stroke (MATCH) ha confrontato il clopidogrel da solo verso la combinazione clopidogrel-aspirina. ${ }^{74}$ Non furono rilevate differenze tra i due trattamenti in termini di efficacia preventiva ma la combinazione aumentò il rischio di emorragie maggiori. Lo studio PROFESS non ha raggiunto i criteri predefiniti per la non inferiorità ma ha mostrato una sovrapponibile frequenza di ictus per la aspirinadipiridamolo verso il clopidogrel. ${ }^{75}$ Non vi sono evidenze che uno dei due trattamenti prevalga sull'altro in termini di prevenzione secondaria.

\section{Anticoagulanti orali}

La efficacia del warfarin nella prevenzione dell'ictus o del TIA di origine cardioembolica è ampiamente documentata. Il warfarin aspirin recurrent stroke study (WARSS) fu effettuato per valutare se la terapia anticoagulante con warfarin fosse anche efficace nel ridurre le recidive di ictus o TIA di origine non cardioembolica. L'international normalized ratio fu mantenuto tra 1,4 e 2,8 e la dose di aspirina usata fu di $325 \mathrm{mg}$. Non furono riscontrate differenze di efficacia tra $i$ due trattamenti nè vi furono differenze nelle complicanze emorragiche. ${ }^{76}$ Nello studio WASID invece, che ha preso in considerazione pazienti con stenosi intracraniche, alla parità di efficacia tra warfarin ed aspirina si è associata una maggiore significativa presenza di complicanze emorragiche con l'anticoagulante..$^{45}$

Stando così le cose, la terapia antiaggregante continua ad essere la terapia di prima scelta per l'ictus o il TIA non cardioembolici.

\section{Terutroban}

Il trial Prevention of cerebrovascular and cardiovascular Events of ischemic origin with teRutroban in patients with a history oF ischemic strOke or tRansient ischeMic attack (PERFORM) ha studiato gli effetti del terutroban, uno specifico antagonista del recettore del trombossano A2, in confronto alla aspirina in pazienti con TIA o ictus ischemico. La efficacia è risultata perfettamente sovrapponibile tra I due trattamenti e lo stesso si può dire per la sicurezza se non si tiene conto di un lieve aumento di sanguinamenti minori con il terutroban. ${ }^{77}$ Lo studio fu interrotto per la futilità dei risultati dal Comitato per il Monitoraggio dei Dati.

\section{Conclusioni}

La prevenzione primaria dell'ictus rappresenta il miglior approccio per la cura dello stesso. Tuttavia la prevenzione primaria registra ritardi significativi, dovuti a molte cause, se ad esempio si guarda alla cura della ipertensione arteriosa nella popolazione. La prevenzione secondaria, dopo un TIA o un episodio ictale, rappresenta dunque un momento cruciale anche se non tempestivo per affrontare gli ulteriori danni che questo evento vascolare può ancora provocare. Le strategie che si debbono adottare comprendono ancora l'approccio ai fattori di rischio quali la pressione arteriosa, il diabete, le dislipidemie, il fumo, la obesità, la sedentarietà. Un ruolo cruciale svolge la terapia antiaggregante e più specifici interventi quali la rivascolarizzazione carotidea.

\section{Quesiti finali}

Due importanti quesiti sono da considerare: i) Quali modalità di valutazione del rischio e di stratificazione prognostica (anche rispetto alle comorbilità esistenti)? (Tabella 1);, ${ }^{2,7}$ ii) Quali azioni da implementare, ritagliate sul singolo paziente? (Tabella 2). 
Tabella 1. Quesito 1.

La valutazione del rischio in un paziente che ha già presentato un TIA si basa sul $\mathrm{ABCD}^{2} \operatorname{score}^{2,7}$

\begin{tabular}{lc}
\hline $\mathbf{A B C \mathbf { D } ^ { 2 } \text { score }}$ & Punteggio \\
\hline Fattore rischio & 1 \\
\hline $\begin{array}{l}\text { Età } \\
>60 \text { aa }\end{array}$ & 1 \\
\hline $\begin{array}{l}\text { Pressione arteriosa } \\
\quad \text { PA sistolica } \geq 140 \mathrm{mmHg} \text { o PA diastolica } \geq 90 \mathrm{mmHg}\end{array}$ \\
\hline Quadro clinico & 2 \\
$\quad$ Ipostenia (debolezza muscolare) di un lato con o senza disturbo di linguaggio & 1 \\
$\quad$ Disturbo del linguaggio senza ipostenia & 2 \\
\hline Durata del TIA & 1 \\
$\quad \geq 60$ min & 1 \\
\hline Da 10 a 59 min & $0-7$ \\
\hline Totale & 1 \\
\hline
\end{tabular}

$\mathrm{ABCD}^{2}$ score: stratificazione del rischio

\begin{tabular}{lllll}
\hline 0-3 rischio di ictus & a 2 giorni & $1,3 \%$ & a $90 \mathrm{gg}$ & $3 \%$ \\
\hline 4-5 rischio di ictus & a 2 giorni & $4,1 \%$ & a $90 \mathrm{gg}$ & $10 \%$ \\
\hline 6-7 rischio di ictus & a 2 giorni & $8,1 \%$ & a $90 \mathrm{gg}$ & $25 \%$
\end{tabular}

L'ospedalizzazione è indicata se $\mathrm{ABCD}^{2}$ score $>5$ o con ripetuti TIA

L'ospedalizzazione è ragionevole nel TIA entro 72 ore:

se $\mathrm{ABCD}^{2}$ score $>3$

se $\mathrm{ABCD}^{2}$ score $0-2$ con incerta diagnosi o con impossibilità a completare indagini entro 48 ore

se $\mathrm{ABCD}^{2}$ score $0-2$ con evento causato da ischemia focale, dimostrabile elettivamente con RM-DWI

Rischio di stroke successivo a TIA (maggiore nelle prime 24 ore): $10 \%$ in $7 \mathrm{gg}$

$12-30 \%$ stroke con storia di TIA pregresso (circa il $25 \%$ nelle ore precedenti)

Trattamento precoce: riduce dell' $80 \%$ il rischio di stroke a $90 \mathrm{gg}$

TIA, attacchi ischemici transitori; PA, pressione arteriosa; RM-DWI, risonanza magnetica-pesata in diffusione.

Tabella 2. Quesito 2.

1. Ospedalizzazione nei casi indicati in funzione dell' $\mathrm{ABCD}^{2}$ score

2. Prevenzione secondaria precoce

TIA: valutazione con $\mathrm{ABCD}^{2}$ score ed inizio terapia antiaggregante subito

Stroke aterotrombotico: inizio terapia antiaggregante entro 48 ore

TEA precoce: entro 2 settimane

3. Prevenzione secondaria cronica

Controllo dei fattori di rischio (ipertensione arteriosa, dislipidemia, diabete, fumo, uso di estrogeni)

Terapia antiaggregante

TEA

4. Terapia

ASA 50-325 mg (secondo esperti $100 \mathrm{mg}$ )

Clopidogrel $75 \mathrm{mg}$

ASA (25 mg) e dipiridamolo (200 mg) bid

TIA, attacchi ischemici transitori; TEA, trombendarteriectomia; ASA, acido acetilsalicilico.

\section{Bibliografia}

1. Lloyd-Jones D, Adams R, Carnethon M, et al. AHA Statistical Update. Heart disease and stroke statistics - 2009 update. A report from the American Heart Association
Statistics Committee and Stroke Statistics Subcommittee. Circulation 2009;119:e21-e181.

2. Rothwell PM, Giles MF, Flossmann E, et al. A simple score $(\mathrm{ABCD})$ to identify individuals at high risk of stroke after transient ischemic attack. Lancet 2005; 366:29-36. 
3. Giles MF, Rothwell PM. Risk of stroke early after transient ischemic attack: a systematic review and metaanalysis. Lancet Neurol 2007;6:1063-72.

4. Chandratheva A, Metha Z, Geraghty OC, et al. Oxford Vascular Study. Population-based study of risk and prediction of stroke in the first few hours after a TIA. Neurology 2009;72:1941-7.

5. Johnston SC, Gress DR, Browner WS, Sidney S. Shortterm prognosis after emergency department diagnosis of TIA. JAMA 2000;284:2901-6.

6. Johnston SC, Rothwell PM, Nguyen-Huynh MN, et al. Validation and refinement of scores to predict very early stroke after transient ischemic attack. Lancet 2007; 369:283-92.

7. Chandratheva A, Geraghty OC, Luengo-Fernandez R, et al. $\mathrm{ABCD} 2$ score predicts severity rather than risk of early recurrent events after transient ischemic attack. Stroke 2010;41:851-6.

8. Coull A, Lovett JK, Rothwell PM. Population based study of early risk of stroke after transient ischemic attack or minor stroke; implications for public education and organisation of services. BMJ 2004;328:326-8.

9. Fairhead JF, Mehta Z, Rothwell PM. Population based study of delays in carotid imaging and surgery and the risk of recurrent stroke. Neurology 2005;65:371-5.

10. Redgrave JN, Coutts SB, Schulz UG, et al. Systematic review of association between the presence of acute ischemic lesions on diffusion-weighted imaging and clinical predictors of early stroke risk after transient ischemic attack. Stroke 2007;38:1482-8.

11. Giles MF, Albers GW, Amarenco P, et al. Addition of brain infarction to the ABCD2 score: a collaborative analysis of unpublished data on 4574 patients. Stroke 2010;41:1907-13.

12. Merwick A, Albers GW, Amarenco P, et al. Addition of brain and carotid imaging to the ABCD2 score to identify patients at early risk of stroke after transient ischemic attack: a multicentre observational study. Lancet Neurol 2010;9:1060-9.

13. Rothwell PM, Giles MF, Chandratheva A, et al. Effect of urgent treatment of transient ischemic attack and minor stroke on early recurrent stroke (EXPRESS study): a prospective population-based sequential comparison. Lancet 2007;370:1432-42.

14. Lavallée PC, Meseguer E, Abboud H, et al. A transient ischemic attack clinic with round-the-clock access (SOS-TIA): feasibility and effects. Lancet Neurol 2007; 6:953-960.

15. Petty GW, Brown RD jr, Whisnant JP, et al. Ischemic stroke subtypes: a population-based study of functional outcome, survival, and recurrence. Stroke 2000;31: 1062-8.

16. Lovett JK, Coull AJ, Rothwell PM. Early risk of recurrence by subtypes of ischemic stroke in populationbased incidence studies. Neurology 2004;62:569-73.

17. Chen ZM, Sandercock P, Pan HC, et al. Indications for early aspirin use in acute ischemic stroke: a combined analysis of 40,000 randomized patients from the Chinese acute stroke trial and the International Stroke Trial. Stroke 2000;31:1240-9.

18. Diener HC, Bogousslavsky J, Brass LM, et al. Aspirin and clopidogrel compared with clopidogrel alone after recent ischemic stroke or transient ischemic attack in high-risk patients (MATCH): randomised, double-blind, placebo-controlled trial. Lancet 2004;364:331-7.

19. Kennedy J, Hill MD, Ryckborst KJ, et al. Fast assessment of stroke and transient ischemic attack to prevent early recurrence (FASTER): a randomized controlled pilot trial. Lancet Neurol 2007;6:961-9.

20. Markus HS, Droste DW, Kaps M, et al. Dual antiplatelet therapy with clopidogrel and aspirin in symptomatic carotid stenosis evaluated using Doppler embolic detection signal detection: the Clopidogrel and Aspirin for reduction of Emboli in Symptomatic Carotid Stenosis (CARESS) trial. Circulation 2005;111:2233-40.

21. Wong KSL, Chen C, Fu J, et al. Clopidogrel plus aspirin versus aspirin alone for reducing embolization in patients with acute symptomatic cerebral or carotid artery stenosis (CLAIR study): a randomized, open-label, blinded-endpoint trial. Lancet Neurol 2010;9:489-97.

22. Rothwell PM, Algra A, Amarenco P. Medical treatment in acute and long-term secondary prevention after transient ischemic attack and ischemic stroke. Lancet 2011;377:1681-92.

23. Algra A, De Schryrer EL, van GJ, et al. Oral anticoagulants versus antiplatelet therapy for preventing further vascular events after transient ischemic attack or minor stroke of presumed arterial origin. Cochrane Database Syst Rev 2006;3 CD001342.

24. Rothwell PM, Eliasziw M, Gutnikov SA, et al. Endarterectomy for symptomatic carotid stenosis in relation to clinical subgroups and timing of surgery. Lancet 2004;363:915-24.

25. Rerkasem K, Rothwell PM. Systematic review of the operative risks of carotid endarterectomy for recently symptomatic stenosis in relation to the timing of surgery. Stroke 2006;40:e564-72.

26. Toole JF, Malinow MR, Chambless LE, et al. Lowering homocysteine in patients with ischemic stroke to prevent recurrent stroke, myocardial infarction, and death: the Vitamin Intervention for Stroke Prevention (VISP) randomized controlled trial. JAMA 2004;291:565-75.

27. Lewington S, Clarke R, Qizilbash N, et al. Age-specific relevance of usual blood pressure to vascular mortality: a meta-analysis of individual data for one million adults in 61 prospective studies. Lancet 2002; 360:1903-13

28. Kearney PM, Whelton M, Reynolds K, et al. Global burden of hypertension: analysis of worldwide data. Lancet 2005;365:217-23.

29. Dahlof B. Prevention of stroke in patients with hypertension. Am J Cardiol 2007;100:17J-24J.

30. Turan TN, Cotsonis G, Lynn MJ, et al. Relationship between blood pressure and stroke recurrence in patients with intracranial arterial stenosis. Circulation 2007;115: 2969-75.

31. Yusuf S, Sleight P, Pogue J, et al. Effects of an angiotensin-converting-enzyme inhibitor, ramipril, on cardiovascular events in high-risk patients. The Heart Outcomes Prevention Evaluation Study Investigators. N Engl J Med 2000;342:145-53.

32. PROGRESS Collaborative Group. Randomized trial of a perindopril based blood-pressure-lowering regimen among 6105 individuals with previous stroke or transient ischaemic attack. Lancet 2001;358:1033-42.

33. Beckett NS, Peters R, Fletcher AE, et al. Treatment of 
hypertension in patients 80 years of age or older. N Engl J Med 2008;358:1887-98.

34. Schrader J, Luders S, Kulschewski A, et al. Mortality and morbidity after stroke - eprosartan compared with nitrendipine for secondary prevention: principal results of a prospective randomized controlled study (MOSES). Stroke 2005;36:1218-26.

35. Diener HC. Preventing stroke: the PROFESS, ONTARGET, and TRASCEND trial programs. J Hypertens 2009;27:S31-6.

36. Rashid P, Leonardi-Bee J, Bath P. Blood pressure reduction and secondary prevention of stroke and other vacular events: a systematic review. Stroke 2003;34:2741-8.

37. Morgan T, Lauri J, Bertram D, Anderson A. Effect of different antihypertensive drug classes on central aortic pressure. Am J Hypertens 2004; 17:118-23.

38. Ichihara A, Kaneshiro Y, Sakoda M, et al. Add-on amlodipine improves arterial function and structure in hypertensive patients treated with an angiotensin receptor blocker. J Cardiovasc Pharmacol 2007;49:161-6.

39. Henry Feugeas MC, De Marco G, Peretti II, et al. Agerelated cerebral white matter changes and pulse-wave encephalopathy: observations with three-dimensional MRI. Magn Reson Imaging 2005;23:929-37.

40. Sundstrom J, Arima H, Jackson R, et al. Effects of blood pressure reduction in mild hypertension a systematic review and metaanalysis. Ann Intern Med 2015; 162:184-91.

41. Zhang H, Thijs L, Staessen JA. Blood pressure lowering for primary and secondary prevention of stroke. Hypertension 2006;48:187-95.

42. Bath PM, Sprigg N. Control of blood pressure after stroke. Hypertension 2006;48:203-4.

43. Wohlfahrta P, Krajcoviechovaa A, Jozifovaa M, et al. Low blood pressure during the acute period of ischemic stroke is associated with decreased survival. J Hyperten 2015;33:339-45.

44. Rothwell PM, Howard SC, Spence JD. Relationship between blood pressure and stroke risk in patients with symptomatic carotid occlusive disease. Stroke 2003; 34:2583-90.

45. Chimowitz MI, Lynn MJ, Howlett-Smith H, et al. Comparison of warfarin and aspirin for symptomatic intracranial arterial warfarin-aspirin symptomatic intracranial disease stenosis. N Engl J Med 2005;352:1305-16.

46. Viscoli C, Brass L, Kernan W, et al. A clinical trial of estrogen-replacement therapy after ischaemic stroke. N Engl J Med 2001;345:1243-9.

47. Prospective Studies Collaboration. Cholesterol, diastolic blood pressure, and stroke. Lancet 1995;346:1647-53.

48. Sacco RL, Benson RT, Kargman DE, et al. High density lipoprotein cholesterol and ischemic stroke in the elderly: the Northern Manhattan Stroke Study. JAMA 2001;285:2729-35.

49. Lindenstrom E, Boysen G, Nyboe J. Influence of total cholesterol, high density lipoprotein cholesterol, and triglycerides on risk of cerebrovascular disease: the Copenhagen City Heart Study. BMJ 1994;309:11-5.

50. Law MR, Wald NJ, Rudnicka AR. Quantifying effect of statins on low density lipoprotein cholesterol, ischemic heart disease, and stroke; systematic review and metaanalysis. BMJ 2003;326:1423-9.

51. Iso H, Jacobs DR, Wentworth D, et al. Serum cholesterol levels and six-year mortality from stroke in 350,977 men screened for the Muliple Risk Factor Intervention Trial. N Engl J Med 1989;320:904-10.

52. Amarenco P, Tonkin AM. Statins for stroke prevention. Disappointment and hope. Circulation 2004;109:III-44III-49.

53. Heart Protection Study Collaborative Group. MRC/BHF Heart protection study of cholesterol lowering with simvastatin in 20,536 high-risk individuals: a randomized placebo-controlled trial. Lancet 2002;360:7-22.

54. Fiorenza AM, Sommariva D, Branchi A. The PROSPER trial. Lancet 2003;361:428.

55. SPARCL Investigators. High-dose atorvastatin after stroke or transient ischemic attack. N Engl J Med 2006;355:549-59.

56. Amarenco P, Labreuche J. Lipid management in the prevention of stroke: review and update meta-analysis of statins for stroke prevention. Lancet Neurol 2009;8:453-63.

57. Sacco R. Extracranial carotid stenosis. N Engl J Med 2001;345:1113-8.

58. ECST Investigators. Interim results for symptomatic patients with severe (70-99\%) or with mild (0-29\%) carotid stenosis. Lancet 1991;337:1235-43.

59. Rothwell PM, Gutnikov SA, Warlow CP. Reanalysis of the final results of the European Carotid Surgery Trial. Stroke 2003;34:514-23.

60. Dietz A, Kerkefeld J, Theron J, et al. Endovascular treatment of symptomatic carotid stenosis using stent placement: Long-term follow-up of patients with a balanced surgical risk/benefit ratio. Stroke 2001;32:1855-9.

61. CAVATAS Investigators. Endovascular versus surgical treatment in patients with carotid stenosis in the Carotid and Vertebral Artery Transluminal Angioplasty Study (CAVATAS): a randomized trial. Lancet 2001; 357:1729-37.

62. Gurm HS, Yadav JS, Fayad P, et al. Long-term results of carotid stenting versus endarterectomy in high risk patients: the Sapphire Study. N Engl J Med 2008;358: 1572-9.

63. Brown MM, Ederle J, Bonati LH, et al. Safety results of the International Carotid Stenting Study. Early outcome of patients randomised between carotid stenting and endarterectomy for symptomatic carotid stenosis. European Stroke Conference 2009, May 22, Stockholm, Sweden.

64. Rothwell PM. Poor outcome after endovascular treatment of symptomatic carotid stenosis: time for a moratorium. Lancet Neurol 2009;8:871-3.

65. Murad MH, Shahrour A, Shah ND, et al. A systematic review and meta-analysis of randomized trials of carotid endarterectomy vs stenting. J Vasc Surg 2011;53: 792-7.

66. Antithrombotic Trialists' Collaboration. Collaborative meta-analysis of randomised trials of antiplatelet therapy for prevention of death, myocardial infarction, and stroke in high risk patients. BMJ 2002;324:71-86.

67. Antithrombotic Trialists' Collaboration. Aspirin in the primary and secondary prevention of vascular disease: a collaborative meta-analysis of individual participant data from randomised trials. Lancet 2009;373:1849-60.

68. Gorelick P, Richardson D, Kelly M, et al. Aspirin and ticlopidine for prevention of recurrent stroke in black patients: a randomized trial. JAMA 2003;289:2947-57. 
69. Diener H, Cunha L, Forbes C, et al. European Stroke Prevention Study - 2. Dipyridamole and acetylsalicylic acid in the secondary prevention of stroke. J Neurol Sci 1996;143:1-13.

70. Halkes PH, van Gijn J, Kappelle LJ, et al. Aspirin plus dipyridamole versus aspirin alone after cerebral ischemia of arterial origin (ESPRIT): randomised controlled trial. Lancet 2006;367:1665-73.

71. CAPRIE Committee. A randomized, blinded, trial of clopidogrel versus aspirin in patients at risk of ischaemic events (CAPRIE). Lancet 1996;348:1329-39.

72. Shinohara Y, Katayama Y, Uchiyama S, et al. Cilostazol for prevention of secondary stroke (CSPS 2): an aspirin-controlled, double-blind, randomized non-inferiority trial. Lancet Neurol 2010;9:959-68.

73. Costa J, Ferro JM, Matias-Guiu G, et al. Triflusal for preventing serious vascular events in people at high risk. Cochrane Database Syst Rev 2005;3:CD004296.

74. Diener HC, Bogousslavsky J, Brass LM, et al. Aspirin and clopidogrel compared with clopidogrel alone after recent ischaemic stroke or transient ischaemic attack in high-risk patients (MATCH): randomised, double-blind, placebo-controlled trial. Lancet 2004;364:331-7.

75. Sacco RL, Diener HC, Yusuf S, et al. Aspirin and extended-release dipyridamole versus clopidogrel for recurrent stroke. N Engl J Med 2008;359:1238-51.

76. Mohr J, Thompson J, Lazar R, et al. A comparison of warfarin and aspirin for the prevention of recurrent ischaemic stroke. N Engl J Med 2001;345:1444-51.

77. Bousser MG, Amarenco P, Chamorro A, et al. A randomized blinded trial of terutroban versus aspirin in patients with cerebral ischemic events: results of the PERFORM study. Lancet 2011;377:2013-22. 


\title{
Prevenzione cardiovascolare secondaria e nefropatia
}

\author{
Dario Manfellotto, ${ }^{1}$ Giorgio Vescovo ${ }^{2}$ \\ ${ }^{1}$ Dipartimento di Medicina Interna, Ospedale Fatebenefratelli, Roma; ${ }^{2}$ Dipartimento di Medicina Interna, Ospedale San Bortolo, \\ Vicenza, Italia
}

\section{Introduzione ed inquadramento epidemiologico}

La malattia renale cronica (MRC) rappresenta un grande problema di salute pubblica, in costante aumento, con un rischio elevato di progressione verso la fase uremica ed il trattamento emodialitico sostitutivo o il trapianto renale. Attualmente vi sono i Italia circa 50.000 pazienti emodializzati, 4000 pazienti in dialisi peritoneale, 20.000 trapiantati renale, 10.000 pazienti incidenti in trattamento dialitico, 5.000.000 affetti da malattia renale cronica, 15 milioni di ipertesi e 8 milioni di diabetici con possibile danno renale.

Le malattie cardiovascolari (MCV) sono frequentemente associate con la MRC, e vi è un rischio elevato di morbosità e mortalità cardiovascolare nei pazienti nefropatici. La MRC è potenzialmente prevenibile e curabile e rappresenta quindi un fattore di rischio prevenibile della MCV. È noto, infatti, che i pazienti nefropatici in genere ammalano e muoiono di complicanze cardiovascolari. La mortalità da $\mathrm{MCV}$ è da 10 a 30 volte più alta nei pazienti dializzati rispetto alla popolazione generale.

Pazienti con MRC devono quindi essere considerati come a maggior rischio di MCV, e quindi le raccomandazioni per il trattamento basate sulla stratificazione del rischio devono tener conto di tale alto rischio.

Secondo la classificazione del Kidney Disease: Improving Global Outcomes (KDIGO) 2012, ${ }^{1}$ in base alla diminuzione del filtrato glomerulare (VFG) [una diminuzione progressiva, da normale $(>90$ $\mathrm{mL} / \mathrm{min} / 1,73 \mathrm{~m}^{2}$ ) fino all'insufficienza renale terminale $\left(<15 \mathrm{~mL} / \mathrm{min} / 1,73 \mathrm{~m}^{2}\right)$ divisa in 6 gradi] la prognosi viene valutata anche in rapporto all'albuminuria, divisa in 3 gradi (A1 normale: $<30 \mathrm{mg} / \mathrm{die}$; A2, mo-

Corrispondente: Dario Manfellotto, Direzione Scientifica Fatebenefratelli, Lungotevere de' Cenci 5, 00186 Roma, Italia. Fax: +39.06 .6837360 .

E-mail: dario.manfellotto@afar.it

Articolo pubblicato secondo la Creative Commons Attribution NonCommercial 3.0 License (CC BY-NC 3.0).

(C) Copyright D. Manfellotto e G. Vescovo, 2015

Licensee PAGEPress, Italy

QUADERNI - Italian Journal of Medicine 2015; 3:364-370 deratamente aumentata: 30-300 mg/die; A3, severa: $>300 \mathrm{mg} / \mathrm{die})$.

Un VFG normale o lievemente diminuito e albuminuria normale hanno un rischio CV basso. Una diminuzione del VFG lieve moderata e un'albuminuria normale hanno un rischio moderato, che diventa però elevato già in presenza di un'albuminuria moderata.

Una diminuzione moderato-severa del VFG (30$44 \mathrm{~mL} / \mathrm{min} / 1,73 \mathrm{~m}^{2}$ ) ha un rischio già elevato, anche con albuminuria normale. Ulteriori diminuzioni del VFG hanno comunque un rischio molto elevato, anche in presenza di albuminuria normale.

Dal punto di vista nosografico, esiste il termine classico di nefroangiosclerosi, che indica quelle condizioni morbose in cui il rene è danneggiato da una permanente ipertensione arteriosa attraverso l'intermediario di una alterazione diffusa del circolo arterioso intrarenale (V. Cagli, 1976).

A sua volta Ascenzi, nel 1976, affermava che con il termine nefrosclerosi si sogliono definire processi di sclerosi renale indotti da vasculopatie arteriose che si sviluppano in istretta connessione con l'arteriosclerosi e l'ipertensione. In tal senso appare giustificata la proposta di sostituire il termine nefrosclerosi con quello più limitato di nefroangiosclerosi. Dette affezioni comprendono l'arteriolonefrosclerosi $o$ nefrosclerosi benigna $o$ rene grinzo primitivo, e la nefrosclerosi maligna $o$ rene grinzo genuino.

Più modernamente, si tende a distinguere la malattia reno-vascolare, ossia una alterazione vascolare a carico dell'arteria renale (o dei suoi rami), prevalentemente di natura aterosclerotica, che può associarsi ad alterazioni della funzione renale e/o ad ipertensione, e la nefropatia ischemica, ossia una alterazione della funzione renale secondaria ad ipoperfusione renale causata da stenosi/ostruzione dell'arteria e/o da stenosi/ostruzione delle arterie intraparenchimali pre-glomerulari. I due termini indicano, quindi, la causa (malattia renovascolare) e l'effetto (nefropatia ischemica).

La coincidenza di danno renale in corso di malattia vascolare sistemica è anche confermata da vari studi ${ }^{2}$ che dimostrano come in corso di coronarografia e di altri esami angiografici, il rilievo di una stenosi arteriosa renale associata ad aterosclerosi coronarica sia presente in più del $50 \%$ dei pazienti.

Pazienti con un danno renale hanno un carico maggiore di malattie vascolari rispetto ai controlli di pari 
età per ogni livello di disfunzione renale. Il rischio è più evidente in età giovanile. Per esempio, un uomo dializzato di 35 anni ha lo stesso rischio cardiovascolare di un uomo di 80 non dializzato.

A questo dato si è associata l'osservazione epidemiologica dell'alta incidenza di cardiopatia ischemica e di malattie cardiovascolari in genere nei pazienti con insufficienza renale cronica, nei quali la mortalità prevalente appare legata alle complicanze cardiovascolari piuttosto che alla insufficienza renale ed alle sue conseguenze dirette. Questa osservazione ha fatto parlare di sindrome cardio-renale ed anche di cardio-renalanemia syndrome, per la frequente presenza di anemia da disordine cronico nei nefropatici e dell'alto rischio ad essa correlato. ${ }^{3-5}$

L'anemia svolge un ruolo importante nello sviluppo della MCV. L'anemia deve essere corretta con differenti target di emoglobinemia in rapporto a differenti sottogruppi di pazienti. È ormai stabilito, comunque, che una completa correzione dell'anemia non si rende necessaria nei pazienti pre-dialisi e in dialisi. ${ }^{3}$

La dislipidemia è molto frequente nella $\mathrm{MRC}$ ed è influenzata sia dalla funzione renale, sia dal grado di proteinuria. Vi è una progressiva riduzione delle concentrazioni di colesterolo totale, colesterolo con lipoproteine ad alta (c-HDL) e bassa densità (c-LDL), con il declino del filtrato glomerulare. $\dot{E}$ anche ben noto che una significativa ipoalbuminemia secondaria ad una grave proteinuria, come per esempio nella sindrome nefrosica, si accompagna a dislipidemia secondaria. ${ }^{6,7}$

Studi condotti nei pazienti emodializzati hanno mostrato una curva ad U nel rapporto fra colesterolemia e mortalità. ${ }^{8}$ Questa associazione poco comprensibile è un esempio di causa inversa: malattie croniche, infiammazione cronica e malnutrizione causano tutte una riduzione della colesterolemia e sono fattori di rischio indipendenti di mortalità.

\section{Premesse fondamentali sui rapporti fra malattia renale cronica e malattie cardiovascolari}

Sui rapporti fra malattia renale cronica e malattie cardiovascolari sono fondamentali alcune premesse:

i) La nefropatia cronica viene suddivisa in cinque stadi secondo la classificazione proposta dalla $\mathrm{Na}$ tional Kidney Foundation;

ii) Una percentuale rilevante (circa il 30\%) dei pazienti ricoverati per sindrome coronarica acuta presenta una riduzione della funzione renale, con un VFG compreso tra 30 e $60 \mathrm{~mL} / \mathrm{min}$;

iii) I pazienti con filtrato glomerulare $<30 \mathrm{~mL} / \mathrm{min}$ presentano una peggiore prognosi intraospedaliera, con una mortalità del $12,2 \%$, dato che risulta significativamente superiore rispetto ai pazienti con valori di filtrato compresi tra 30 e $60 \mathrm{~mL} / \mathrm{min}$ (mortalità del 5,5\%) e ai pazienti con funzione renale normale (mortalità dell' $1,4 \%$ );

iv) La presenza di valori di filtrato glomerulare $<60$ $\mathrm{mL} / \mathrm{min}$ si associa ad una maggiore incidenza di eventi cardiovascolari sfavorevoli dopo sindrome coronarica acuta;

v) Le linee guida nazionali ed internazionali sono concordi nel raccomandare la stima del filtrato glomerulare mediante la formula MDRD (modification of diet in renal disease) in tutti i pazienti affetti da cardiopatia ischemica. Per questa particolare finalità sono disponibili diversi calcolatori on-line (www.mdrd.com, www.nkdep.nih.gov);

vi) La nefropatia cronica (filtrato glomerulare $<60$ $\mathrm{mL} / \mathrm{min}$ ) si associa alla possibile presenza di anomalie del metabolismo minerale ed osseo, che attualmente sono indicate nella letteratura internazionale come chronic kidney disease-mineral and bone disorder. Tale condizione viene definita come la combinazione di: anomalie del metabolismo del calcio (ipocalcemia) e del fosforo (iperfosforemia), anomalie del metabolismo del paratormone (iperparatiroidismo secondario) e della vitamina D (deficit vitaminico).

La MRC interessa circa il 35\% dei pazienti di oltre 65 anni di età, ed è una delle più frequenti comorbidità osservate in ospedale, particolarmente nei pazienti con $\mathrm{MCV}$ acuta. Anche la degenza media nei pazienti con MRC è del $35 \%$ più lunga rispetto agli altri pazienti ricoverati, ma senza MRC.

Pazienti con MRC che sono ricoverati in ospedale con STEMI o nSTEMI ricevono in media procedure meno evidence based rispetto ai pazienti senza danno renale. Anche alla dimissione dall'ospedale, viene previsto un programma diagnostico terapeutico meno completo per il follow-up della loro cardiopatia.

Un danno renale acuto (acute kidney injury [AKI]) colpisce circa il $20 \%$ dei pazienti ricoverati in urgenza. Quelli maggiormente a rischio sono i pazienti con MRC preesistente, con diabete o che hanno effettuato procedure con mezzo di contrasto, come coronarografia o angiografie, o tomografia computerizzata. In un terzo dei casi, la AKI è prevenibile ed evitabile. ${ }^{9}$

Le alterazioni vascolari hanno carattere progressivo ed incidono negativamente sulla prognosi, favorendo ulteriori eventi cardiovascolari avversi.

Tutti i pazienti con pregressa sindrome coronarica acuta devono essere valutati per la possibile presenza di disfunzione renale, mediante la stima del filtrato glomerulare con la formula MDRD.

È anche necessaria una valutazione della proteinuria in quanto la sua presenza modifica in modo sostanziale il profilo di rischio cardiovascolare residuo e di progressione del danno renale.

Come esame di screening è sufficiente l'esecuzione di un esame chimico-fisico delle urine (dipstick urinario), riservando il dosaggio della proteinuria sulle urine delle $24 \mathrm{~h}$ a casi selezionati. Si sottolinea che anche la presenza un solo + al dipstick aumenta significativamente il rischio di successivi eventi avversi nei pazienti con malattia coronarica. In caso di valori di filtrato glomerulare $<60 \mathrm{~mL} / \mathrm{min}$, la valutazione deve essere ripetuta dopo 3 mesi. Se il con- 
trollo conferma la presenza di valori $<60 \mathrm{~mL} / \mathrm{min}$, si dovrà avviare una valutazione complessiva che tenga conto della possibile presenza delle anomalie del metabolismo minerale ed osseo. ${ }^{8}$

Si raccomanda che in tutti i pazienti con MRC, in ognuno dei cinque stadi, venga raccolta una storia clinica che tenga conto dei fattori di rischio $\mathrm{CV}$, allo scopo di porre in atto tutte le misure di prevenzione $\mathrm{CV}$ secondaria: i) angina e infarto del miocardio; ii) pregressa rivascolarizzazione coronarica; iii) ictus o ischemia cerebrale transitoria; iv) pregressa chirurgia carotidea o angioplastica; v) malattia vascolare periferica o pregresso intervento; vi) insufficienza cardiaca; vii) aritmie (sopraventriculari e ventricolari); viii) diabete; ix) etnìa.

\section{Screening per l'albuminuria}

La proteinuria ha un duplice significato. Nei pazienti con nefropatia proteinurica, la velocità di progressione dell'insufficienza renale è determinata dal livello di pressione e dall'entità della proteinuria, e pertanto la riduzione sia della pressione sia della protenuria ha un effetto benefico sul rischio renale. La proteinuria è anche un marker di danno vascolare sistemico e per tale motivo la terapia per ridurre la proteinuria e controllare l'ipertensione mira a migliorare il rischio cardiovascolare e a ridurre la mortalità. ${ }^{10}$

Molti pazienti presentano insufficienza renale associata a complicanze cardiovascolari senza che sia noto un precedente danno renale. Si raccomanda pertanto che le misure di prevenzione secondaria nei pazienti con MRC vengano estese ai soggetti che sono a rischio di insufficienza renale progressiva, anche se non hanno mai sofferto di una nefropatia nota. Questi soggetti possono essere identificati con lo screening per l'albuminuria. Un'albuminuria elevata non soltanto è un indicatore prognostico negativo di malattia renale, ma anche di malattia cardiovascolare. Oltre ai pazienti diabetici, sono a rischio di albuminuria anche gli ipertesi, gli obesi e i fumatori. ${ }^{11}$

Le terapie che si sono rivelate efficaci per ridurre l'escrezione di albumina, come gli ACE inibitori (ACE-I), gli antagonisti recettoriali dell'angiotensina II (ARBs) e le statine, dovrebbero essere prescritte precocemente in tali pazienti, allo scopo di prevenire lo sviluppo di una malattia renale manifesta e delle sue conseguenti complicanze cardiovascolari. ${ }^{12}$

\section{Gli studi clinici disponibili e le evidenze}

La prevenzione secondaria della perdita progressiva della funzione renale in paziente con preesistente nefropatia prevede la dieta ipoproteica, la restrizione di sodio e l'impiego di farmaci attivi sul sistema renina-angiotensina, come gli ACE-I e gli ARBs, insieme ad altri antipertensivi, se necessario.

L'efficacia di vari approcci nella prevenzione primaria dello sviluppo e della progressione dell'albuminuria è al contrario meno chiara.
Sebbene vi siano alcuni studi secondo i quali la progressione dell'albuminuria può essere arrestata nei soggetti diabetici, grazie ad uno stretto controllo della glicemia e l'uso di ACE-I e di ARBs, i trial di prevenzione primaria che dimostrano la possibilità di prevenire la progressione dalla fase di normoalbuminuria alla microalbuminuria sono molto scarsi.

Uno stretto controllo della glicemia può rallentare l'aumento dell'albuminuria sia nel diabete di tipo 1 che in quello di tipo 2, e rimandare l'insorgenza di una nefropatia diabetica conclamata.

Nell'EUCLID study ${ }^{13}$ la terapia con un ACE-I ha prevenuto l'aumento della albuminuria, sebbene questo effetto benefico risultasse più evidente $(49,7 \%)$ nei soggetti con microalbuminuria rispetto ai soggetti con escrezione di albumina di entità normale-alta.

Sono stati pubblicati anche studi sugli effetti dei farmaci ipolipemizzanti sull'albuminuria e sulla reno protezione nel diabete mellito. L'uso di statine nei pazienti con MRC (stadio 1-4) è stato ampiamente studiato e si è rivelato efficace e sicuro nel ridurre la mortalità cardiovascolare. ${ }^{14}$

Le statine sembrano conferire renoprotezione nei diabetici dislipidemici normotesi o ipertesi , così come nella dislipidemia familiare. ${ }^{15}$ questa riduzione della escrezione di albumina appare in larga parte indipendente dalla riduzione del colesterolo LDL. Anche la riduzione dei trigliceridi con gemfibrozil nei diabetici di tipo 2 riduce l'albuminuria. ${ }^{16}$

Alla luce di quanto affermato finora, si sottolinea nuovamente l'importanza di effettuare una prevenzione secondaria non soltanto in pazienti con nefropatia già nota, ma anche in quelli che sono a rischio di insufficienza renale progressiva, compresi quelli con albuminuria normale-alta.

La prognosi dei pazienti con MRC dopo un infarto acuto non è buona ed è correlata con il grado di coinvolgimento renale. ${ }^{17}$

Come già accennato, vi è anche una forte evidenza che in questo gruppo di pazienti vi sia un sottoutilizzo delle terapie basate sulle linee guida, ma questo dato non sembra essere sufficiente per spiegare l'aumento del rischio conferito dalla malattia renale. ${ }^{18}$

\section{Prevenzione farmacologica}

Il ruolo dell'aspirina in prevenzione primaria è ancora controverso in tutti i pazienti, tranne che in quelli ad alto rischio ma con normale funzione renale, in ragione del rischio di stroke emorragico e del rischio relativamente basso di eventi vascolari, rispetto ai pazienti in prevenzione secondaria. Per quanto riguarda però $\mathrm{i}$ pazienti con danno renale, il beneficio sembra controbilanciare il rischio in prevenzione secondaria.

Alcuni studi hanno sollevato dubbi sulla sicurezza della terapia con aspirina nell'insufficienza renale. Lo studio DOPPS-data ${ }^{19}$ mostra che l'aspirina riduce lo stroke ma non la cardiopatia ischemica. In effetti, vi 
era un'alta incidenza di infarto del miocardio nei pazienti in terapia con aspirina, anche se erano presenti fattori confondenti difficili da interpretare.

Altri studi non segnalano problemi di sicurezza. ${ }^{20}$ Non vi sono studi controllati randomizzati sull'uso dell'aspirina nei pazienti con MRC, per cui l'opinione corrente è che l'aspirina vada prescritta a bassa dose (75-150 mg) in tale categoria di pazienti. ${ }^{21}$ Anche per clopidogrel mancano studi clinici randomizzati nei nefropatici, sebbene venga ne segnalata la sicurezza e la possibilità quindi di un impiego in prevenzione secondaria nei pazienti intolleranti all'aspirina. ${ }^{22}$ Anche l'associazione di aspirina e clopidogrel o ticagrelor viene suggerita in prevenzione secondaria fino a 12 mesi dopo angioplastica e nel non STEMI. ${ }^{23}$

Il target della emoglobina glicata $(\mathrm{HbA} 1 \mathrm{c})$ in tutti i pazienti con MRC, dializzati e trapiantati deve essere fra $6,5 \%(48 \mathrm{mmol} / \mathrm{mmol} / \mathrm{HbA} 0)$ e $7,5 \%(58 \mathrm{mmol} /$ $\mathrm{mmol} / \mathrm{HbA} 0)$.

Deve essere ottenuta una riduzione del colesterolo totale del $25 \%$ rispetto al valore basale, e del colesterolo LDL, a digiuno, del $30 \%$.

A tale scopo, le statine devono essere sempre considerate nel trattamento dei pazienti con MRC, in ogni stadio della MRC (stadio 1-5), ed anche nei pazienti dializzati e dopo trapianto di rene.

Le statine non devono essere sospese neanche all' $i-$ nizio della terapia sostitutiva renale (dialisi o trapianto).

Sussiste quindi la raccomandazione che sia i pazienti con MRC stadio 1-3, sia quelli agli stadi 4 e 5 (compresi i soggetti in dialisi o con trapianto di rene) con storia di angina stabile, sindrome coronarica acuta, infarto del miocardio, stroke, arteriopatia periferica, o che siano sottoposti a chirurgia coronarica o rivascolarizzazione angiografia, debbano essere trattati con aspirina, ACE-I, $\beta$-bloccante e statina, a meno che non presentino controindicazioni all'uso di tali farmaci.

Aspirina e clopidogrel sono indicati fino a 12 mesi dopo angioplastica e stenting e nel NSTEMI, ma possono provocare un aumento del rischio di sanguinamenti.

Aspirina è indicata per la prevenzione secondaria delle malattie vascolari nell'insufficienza renale ma non per quella primaria.

Le dosi di ACE-I e $\beta$-bloccanti devono essere adattate fino alla massima dose efficace tollerata.

La MRC viene considerata come un fattore di rischio equivalente di cardiopatia ischemica.

Questo ha portato al definire come obiettivo primario la riduzione di LDL colesterolo. Il colesterolo non-HDL deve invece essere considerato il secondo obiettivo nella gestione di una dislipidemia mista.

L'algoritmo di trattamento deve essere basato sul VFG. Devono essere preferiti farmaci eliminati prevalentemente per via epatica (fluvastatina, atorvastatina, pitavastatina, ezetimibe). Le statine, metabolizzate dal CYP3A4 possono portare ad effetti indesiderati dovuti ad interazioni farmaco-farmaco e pertanto con esse è necessaria una particolare cautela.
Le statine sono generalmente ben tollerate a dosi moderate in soggetti con MRC stadio 1-2. Problemi di sicurezza e adattamento della dose diventano importanti in stadi più avanzati di danno renale (stadi 35) e gli effetti indesiderati sono in genere legati alla dose e dovuti all'aumento della concentrazione plasmatica del farmaco impiegato.

Riassumendo il ruolo delle statine nella malattia renale, possiamo dire che le situazioni sono diverse per quanto concerne gli stadi 1-4 e lo stadio 5 (compresi i pazienti dializzati).

Vi sono due revisioni Cochrane del 2013 che riguardano questi argomenti.

La prima sulla malattia renale stadio 2-4 sostanzialmente afferma che l'insufficienza renale cronica (IRC) è frequente a causa dell'invecchiamento della popolazione. Le complicazioni cardiovascolari (CV) sono la principale causa di mortalità nei pazienti affetti da IRC. In circa il $60 \%$ dei pazienti in IRC (stadi 3-4; VFG $<60$ $\mathrm{mL} / \mathrm{min}=$ pre-dialisi) è presente un'ipercolesterolemia, responsabile delle complicazioni $\mathrm{CV}$ e fattore di rischio nella progressione della malattia renale. Benché le statine siano frequentemente prescritte per il trattamento dell'ipercolesterolemia in pazienti affetti da IRC, pochi sono i dati sulla loro efficacia clinica. Questa revisione sistematica mira a determinare $i$ benefici ed i rischi dell'assunzione quotidiana delle statine nei pazienti predializzati, con o senza comorbidità CV:

i) Sul profilo lipidico;

ii) Sulla mortalità (totale e CV);

iii) Sulla progressione dell'insufficienza renale;

iv) Sugli effetti secondari.

Sono stati identificati ventisei studi (25.017 pazienti affetti da IRC) che confrontano l'assunzione di statine al placebo. L'assunzione quotidiana di statine:

i) Riduce il colesterolo totale $\mathrm{di}-1,1 \mathrm{mmol} / \mathrm{L}$ (intervallo di confidenza [IC] 95\%: $-1,3$ a $-0,9$ ) e il colesterolo LDL di $-1,1 \mathrm{mmol} / \mathrm{L}$ (IC 95\%: $-1,3$ a $-0,9)$;

ii) Diminuisce il rischio di mortalità totale (rischio relativo [RR]: 0,8 ; IC 95\%: 0,7-0,9) e di mortalità CV (RR: 0,8; IC 95\%: 0,7-0,9);

iii) Diminuisce l'escrezione urinaria di proteine $(-0,7$ $\mathrm{g} / 24 \mathrm{~h}$; IC 95\%: -0,95 a -0,5) ma non aumenta la clearance della creatinina $(1,5 \mathrm{~mL} / \mathrm{min}$, IC 95\%:$2,3$ a 5,3$)$;

iv) Non aumenta il rischio di effetti secondari (rabdomiolisi, diminuzione della funzione renale).

Da ricordare che soltanto le statine sono considerate in questa revisione. Pochi erano inoltre gli studi di elevata qualità (informazioni mancanti relative alla randomizzazione, alla cecità ed al metodo di analisi dell'intention to treat); il numero di pazienti era limitato e il periodo di follow up era breve. Lo studio Pravastatine pooling project era quello che aveva un'influenza maggiore sui risultati della metanalisi. Per certi risultati (clearance della creatinina e proteinuria) pochi sono gli studi inclusi nella metanalisi. I dati inclusi in questa revisione provengono spesso da 
analisi di sottogruppi inclusi in grandi studi clinici.

Le statine diminuiscono il rischio di mortalità totale e di mortalità CV e permettono un migliore controllo lipidico nei pazienti affetti da IRC. Malgrado non abbiamo impatto sulla filtrazione glomerulare, sembrano diminuire l'escrezione urinaria di proteine ed avere così un potenziale effetto nefro-protettivo. Le statine causano pochi effetti secondari. A fronte di questi risultati, gli autori concludono che possono essere prescritte nei pazienti affetti da IRC.

Anche per i pazienti dializzati c'è una revisione Cochrane del 2013 che suggerisce che l'uso di statine non migliora la prognosi cardiovascolare.

Gli eventi cardiovascolari sono molto comuni tra i pazienti con malattia renale cronica e decisamente più frequenti nei pazienti sottoposti a dialisi. In considerazione di quest'alto numero di eventi cardiovascolari si è cercato di trovare un legame tra un aumento del colesterolo totale o del c-LDL (responsabili nella popolazione generale delle lesioni aterosclerotiche) e la morte cardiovascolare, ma i risultati sono stati conflittuali, come pure non si sono riscontrate relazioni convincenti sul fatto che le statine (parliamo sempre di malati sottoposti a dialisi) migliorino gli outcomes. E una review sistematica con metanalisi di Palmer e coll. ${ }^{24}$ pubblicata nella Cochrane Database of Systematic Reviews ha confermato questi dati: l'uso delle statine non migliora la mortalità totale e cardiovascolare nei pazienti dializzati.

La metanalisi è stata ripresa da Ali Olyaei su Evid Based Med, il quale, nel confermare le conclusioni di Palmer, ricorda che essendo il primum non nocere la pietra angolare di ogni intervento terapeutico, vista la mancanza di benefici clinici e stante il potenziale di effetti avversi gravi, è giunto il momento di non consigliare le statine nei pazienti dializzati e di investire in altre soluzioni per cercare di ridurre l'elevato onere di eventi cardiovascolari in questi pazienti.

Nei pazienti sottoposti a dialisi, accanto ai tradizionali fattori di rischio per la MCV, quali l'ipertensione, il diabete, l'iperlipidemia ve ne sono altri non tradizionali come l'anemia, le calcificazioni, la proteinuria, lo stress ossidativo e le alterazioni della coagulazione correlate alla uremia. Ed esse verosimilmente rappresentano la quota prevalente: infatti nei pazienti dializzati solo il $25 \%$ dei decessi è legato alle conseguenze delle lesioni aterosclerotiche tradizionali, mentre il restante $75 \%$ è secondario a patologie aritmiche e ad insufficienza cardiaca, che non sono strettamente correlabili alle alterazioni del profilo lipidico.

La metanalisi di Palmer è in linea con questi dati: negli 8289 pazienti in dialisi studiati per valutare l'efficacia di un trattamento con statine per ridurre la morte cardiovascolare e/o la mortalità per tutte le cause l'uso di questi farmaci non ha dato nessun vantaggio rispetto al placebo o a nessun trattamento. In particolare:

i) Non ha avuto alcun effetto significativo sui principali eventi cardiovascolari (RR: 0,95, 95\% IC 0,88-1,03); ii) Non ha dimostrato alcun beneficio della mortalità totale (RR: 0,96, 95\% IC 0,9-1,02);

iii) Non è stato associato ad una riduzione significativa della morte cardiovascolare (RR: 0,94, 95\% IC 0,84-1,06);

iv) Non ha determinato differenze nell'incidenza di ictus e o infarto miocardico acuto (RR: 1,29, 95\% IC 0,96-1,72).

Olyaei, nel commento alla metanalisi, osserva che i suoi risultati sono coerenti con i risultati di precedenti studi clinici randomizzati ${ }^{25,26}$ e con un'altra recente meta-analisi sull'argomento, ${ }^{27}$ ben in linea con le motivazioni patogenetiche sovraesposte. Inoltre, pur non essendo riportata l'incidenza di effetti collaterali connessi con l'utilizzo delle statine in questo particolare setting di pazienti, il dato non va in nessun modo dimenticato, vista la maggiore incidenza della miopatia nei pazienti con funzione renale compromessa che, tra l'altro, necessitano di elevate posologie delle medesime.

Vi è una crescente evidenza che i fibrati fanno aumentare la creatininemia e l'omocisteina. Gli effetti del fenofibrato sono maggiori di quelli del gemfibrozil. Poiché i fibrati non hanno effetti sull'escrezione urinaria di creatinina, ciò costituisce un problema clinico pratico nella stima del VFG. Fenofibrato è peraltro non dializzabile e non dovrebbe essere prescritto al di sotto di un VFG di $50 \mathrm{~mL} / \mathrm{min} / 1,73 \mathrm{~m}^{2}$.

La dose di gemfibrozil raccomandata è di 600 $\mathrm{mg} /$ die se VFG è $60 \mathrm{~mL} / \mathrm{mL} / 1,73 \mathrm{~m}^{2}$, ma il farmaco va evitato se VFG è inferiore a $15 \mathrm{~mL} / \mathrm{min} / 1,73 \mathrm{~m}^{2} .{ }^{28}$

Le linee guida European Society of Cardiology (ESC)/European Atherosclerosis Society (EAS) ${ }^{28}$ riconoscono la MRC come rischio equivalente di malattia coronarica. In questi pazienti la riduzione del c-LDL viene raccomandata come target primario della terapia (classe I, livello A) e l'impiego di statine dovrebbe essere preso in considerazione come monoterapia o con altri farmaci per ottenere un LDL $<70$ mg\% (classe IIa, livello B). Sempre con classi di evidenza elevate (IA) ma con livelli di evidenza variabili si raccomandano l'abbassamento del c-LDL per ridurre il rischio CV e l'utilizzo delle statine che proteggono contro lo sviluppo di end stage renal disease e hanno un effetto protettivo sulla proteinuria.

Le linee guida ESH/ESC 2013/ESC 2013 ci forniscono anche delle indicazioni sul trattamento dei pazienti ipertesi con nefropatia. ${ }^{29}$

Come raccomadazione A e livello di evidenza A si consigliano i farmaci che agiscono sul sistema renina angiotensina (RAS) come i più efficaci nel ridurre le proteinuria; per ottenere i target pressori di solito bisogna comunque associarli (in particolare a diuretici o calcioantagoniati). Sul target pressorio nei pazienti con insufficienza renale non c'è unanimità: si accetta quello di una riduzione della pressione arteriosa sistolica $<140$ $\mathrm{mmHg}$, ma con classe IIa e livello di evidenza B.

Da evitare l'associazione di due bloccanti il RAS e l'uso di antagonisti dell'aldosterone (rischio elevato di iperkaliemia). 


\section{Prevenzione non farmacologica: ruolo dell'alimentazione dell'attività fisica e della riabilitazione}

L'obesità è un fattore di rischio per lo sviluppo di malattia renale e programmi di riduzione di peso possono aiutare i pazienti in fase pre-dialitica a ridurre la proteinuria, la pressione arteriosa e la perdita di funzione renale. ${ }^{30}$

L'attività fisica unita alla prescrizione di farmaci per l'obesità e inteventi dietetici e la chiruriga bariatrica per i gravi obesi possono anche aiutare a migliorare la candidabilità al trapianto di rene. Pochi sono i dati sulla dialisi peritoneale.

\section{Pazienti a rischio cardiovascolare elevato/ molto elevato per contemporanea presenza di danno cardiaco e renale}

È chiaro che quando si considerano pazienti a rischio cardiovascolare elevato/ molto elevato si deve far riferimento quindi a chi ha già in atto una decurtazione della funzione renale secondo la definizione del KDIGO già precedentemente illustrata e coloro che hanno avuto un danno cardiaco che abbia portato ad una compromissione della funzione cardiaca stessa. La terza categoria di pazienti sono i diabetici in cui si rileva un danno renale.

Questi pazienti possono venire inquadrati come portatori di sindrome cardiorenale (CRS), definita da Ronco $^{31}$ come un disordine fisiopatologico del cuore e dei reni nel corso del quale la disfunzione acuta o cronica di uno dei due organi può indurre una disfunzione acuta o cronica dell'altro organo.

La CRS viene classificata in 5 sotto-tipi: sindrome cardiorenale acuta (tipo I) e cronica (tipo II); sindrome renocardiaca acuta (tipo III) e cronica (tipo IV).

Lo scompenso acuto e cronico può essere responsabile di queste due forme.

Esiste poi una sindrome cardiorenale secondaria ad altre patologie, quali diabete e sepsi (tipo V).

Questi pazienti debbono essere considerati a rischio CV elevato/molto elevato e quindi essere trattati come tali. In particolare devono essere considerate le seguenti strategie:

i) Prevenzione della progressione del danno cardiaco; ii) Prevenzione della progressione del danno renale; iii) Trattamento aggressivo dei fattori di rischio CV; iv) Trattamento del diabete e sue complicanze.

La prevenzione della progressione del danno cardiaco deve essere incentrata sulle misure che prevengono la progressione dello scompenso ed il miglioramento dei sintomi legati allo scompenso stesso. ${ }^{32}$

La prevenzione della progressione del danno renale deve avvenire secondo quanto precedentemente illustrato.

I fattori di rischio vanno trattati secondo quanto stabilito dalle linee guida europee ESC 2007.
Il diabete va trattato in accordo con gli standard di cura AMD-SID (Associazione Medici DiabetologiSocietà Italiana di Diabetologia) del 2014 che prevedono anche l'uso dei farmaci di ultima generazione quali inibitori DPP4 ed analoghi GLP1.

Naturalmente vanno corrette le comorbidità quali ad esempio l'anemia.

Da tenere in considerazione l'uso di farmaci che potenzialmente possono aggravare la funzione renale quali diuretici specie tiazidici, gli antagonisti dell'aldosterone, gli ACE-I e gli ARBs.

Infine, va accennato che sempre più si afferma il concetto di interazione e comunicazione tra apparati, che nel nostro caso non coinvolge solo reni e cuore, ma reni, cuore, polmoni, fegato. Oltre alla sindrome cardiorenale (e/o alla sindrome renocardiaca), alla sindrome epatorenale (che però esula lo scopo di questa breve rassegna), si parla ormai anche di una sindrome cardio-polmonare-renale.

Considerati l'elevata mortalità e l'alto tasso di complicanze nel decorso clinico dei ricoveri dei pazienti che presentano un coinvolgimento di tutti e tre gli organi nel processo patologico, è necessario un approccio integrato per l'identificazione dei meccanismi fisopatologici coinvolti, l'individuazione di biomarcatori, che richiede la collaborazione di esperti appartenenti alle diverse discipline al fine di elaborare futuri provvedimenti che possano migliorare la situazione clinica e la sopravvivenza di questi pazienti. ${ }^{33}$

\section{Conclusioni}

In conclusione, è possibile riassumere il discorso su prevenzione cardiovascolare secondaria e nefropatia in 3 punti essenziali:

- Stratificazione prognostica in quanto pazienti già a rischio elevato/molto elevato.

Proposta da 1 a 5 (1 punto per ogni item): i) severità compromissione renale (stage KDIGO e proteinuria); ii) danno d'organo specie cardiaco (ecocardioNTpro-BNP); iii) fattori di rischio CV (ipertensione, diabete, c-LDL, fumo, età, sesso); iv) altre comorbidità (anemia, broncopneumopatia cronica ostruttiva, vasculopatia periferica, ecc.); v) frailty.

- Gerarchizzazione del trattamento e della sua aggressività.

- Rivalutazione clinica e strumentale a seconda della severità della prognosi 1 mese 3 mesi 6 mesi 1 anno con: i) esame clinico, fattori rischio e comorbidità; ii) funzione renale, NTproBNP, emocromo, profilo lipidico, glicidico; iii) aderenza terapeutica.

\section{Bibliografia}

1. Kidney Disease: Improving Global Outcomes (KDIGO) CKD Work Group. KDIGO clinical practice guideline for the evaluation and management of chronic kidney disease. Kidney Intern Suppl 2013;3:1-150. 
2. Harding MB, Smith LR, Himmelstein SI, et al. Renal artery stenosis: prevalence and associated risk factors in patients undergoing routine cardiac catheterization. J Am Soc Nephrol 1992;2:1608-16.

3. Iaina A, Silverberg DS, Wexler D, Iaina Nomy L. The cardio-renal anemia syndrome. Med Pregl 2007;60:145-50.

4. Locatelli F, Covic A, Eckardt K, et al. Anaemia management in patients with chronic kidney disease: a position statement by the Anaemia Working Group of European Renal Best Practice (ERBP). Nephrol Dial Transplant 2009;24:348-54.

5. von Haehling S, Anker SD. Cardio-renal anemia syndrome. Contrib Nephrol 2011;171:266-73.

6. Shurraw S, Tonelli M. Statins for treatment of dyslipidemia in chronic kidney disease. Perit Dial Int 2006;26: 523-39.

7. Hull RP, Goldsmith DJA. Nephrotic syndrome in adults. BMJ 2008;336:1185-9.

8. Goldwasser P, Mittman N, Antignani A, et al. Predictors of mortality in hemodialysis patients. J Am Soc Nephrol 1993;3:1613-22.

9. Colivicchi F, Di Roma A, Uguccioni M, et al. Prevenzione cardiovascolare secondaria dopo sindrome coronarica acuta nella pratica clinica - Documento di Consenso delle Società Scientifiche di Medicina Cardiovascolare e Medicina Interna della Regione Lazio. G Ital Cardiol 2010;11:3S-29S.

10. Bakris GL, Williams M, Dworkin L, et al. Preserving renal function in adults with hypertension and diabetes: a consensus approach. National Kidney Foundation Hypertension and Diabetes Executive Committees Working Group. Am J Kidney Dis 2000;36:646-61.

11. Telmer S, Christiansen JS, Andersen AR, et al. Smoking habits and prevalence of clinical diabetic microangiopathy in insulin dependent diabetes. Acta Med Scand 1984;215:63-8.

12. De Jong PE, Brenner BM. From secondary to primary prevention of progressive renal disease: the case for screening for albuminuria. Kidney Int 2004;66:2109-18.

13. [No authors listed]. Randomised placebo-controlled trial of lisinopril in normotensive patients with insulin-dependentdiabetes and normoalbuminuria or microalbuminuria. The EUCLID Study Group. Lancet 1997;349: 1787-92.

14. Navaneethan SD, Nigwekar SU, Perkovic V, et al. HMGCoA reductase inhibitors (statins) for dialysis patients. Cochrane Database Syst Rev 2009;(3):CD004289.

15. Sinzinger H, Kritz H, Furberg CD, et al. Atorvastatin reduces microalbuminuria in patients with familial hypercholesterolemia and normal glucose tolerance. Med Sci Monit 2003;9:188-92.

16. Smulders YM, van Eeden AE, Stehouwer CD, et al. Can reduction in hypertriglyceridaemia slow progression of microalbuminuria in patients with non insulin-dependent diabetes mellitus? Eur J Clin Invest 1997;27:9971002 .

17. Wright RS, Reeder GS, Herzog CA, et al. Acute myocardial infarction and renal dysfunction: a high-risk combination. Ann Intern Med 2002;137:563-70.

18. Peterson PN, Ambardekar AV, Jones PG, et al. Increased mortality among survivors of myocardial infarction with kidney dysfunction: the contribution of gaps in the use of guideline-based therapies. BMC Cardiovasc Disord 2009;9:29.
19. Ethier J, Bragg-Gresham JL, Piera L, et al. Aspirin prescription and outcomes in hemodialysis patients: the Dialysis Outcomes and practice Patterns Study (DOPPS). Am J Kidney Dis 2007;50:602-11.

20. Baigent C, Blackwell L, Collins R, et al. Aspirin in the primary and secondary prevention of vascular disease: collaborative meta-analysis of individual participant data from randomised trials. Lancet 2009;373:1849-60.

21. Notaro LA, Usman MH, Burke JF, et al. Secondary prevention in concurrent coronary artery, cerebrovascular, and chronic kidney disease: focus on pharmacological therapy. Cardiovasc Ther 2009;27:199-215.

22. Dember LM, Beck GJ, Allon M, et al. Effect of clopidogrel on early failure of arteriovenous fistulas for hemodialysis: a randomized controlled trial. JAMA 2008;299:2164-71.

23. Keltai M, Tonelli M, Mann JFE, et al. Renal function and outcomes in acute coronary syndrome: impact of clopidogrel. Eur J Cardiovasc Prev Rehabil 2007;14: 312-31.

24. Palmer SC, Navaneethan SD, Craig JC, et al. HMG CoA reductase inhibitors (statins) for dialysis patients: a systematic review and meta-analysis. Cochrane Database Syst Rev 2013;(9):CD004289.

25. Wanner C, Krane V, März W, et al. Atorvastatin in patients with type 2 diabetes mellitus undergoing hemodialysis. N Engl J Med 2005;353:238-48.

26. Fellström BC, Jardine AG, Schmieder RE, et al. Rosuvastatin and cardiovascular events in patients undergoing hemodialysis. N Engl J Med 2009;360:1395-407.

27. Hou W, Jicheng LV, Perkovic V, Yang L, et al. Effect of statin therapy on cardiovascular and renal outcomes in patients with chronic kidney disease: a systematic review and meta-analysis. Eur Heart J 2013;34:1807-17.

28. European Association for Cardiovascular Prevention \& Rehabilitation; Reiner Z, Catapano AL, et al. ESC/EAS Guidelines for the management of dyslipidaemias: the Task Force for the management of dyslipidaemias of the European Society of Cardiology (ESC) and the European Atherosclerosis Society (EAS). Eur Heart J 2011;32:1769-818.

29. Mancia G, Fagard R, Narkiewicz K, et al. 2013 ESH/ESC Guidelines for themanagement of arterial hypertension TheTask Force for the management ofarterial hypertension of the European Society ofHypertension (ESH) and of the European Society of Cardiology (ESC). J Hypertens 2013;31:1281-357.

30. Kambham N, Markowitz GS, Valeri AM, et al. Obesityrelated glomerulopathy: an emerging epidemic. Kidney Int 2001;59:1498-509.

31. Ronco C, McCullough P, Anker SD, et al. Cardio-renal syndromes: report from the consensus conference of the acute dialysis quality initiative. Eur Heart J 2010; 31:703-11.

32. McMurray JJ, Adamopoulos S, Anker SD, et al. ESC Guidelines for the diagnosis and treatment of acute and chronic heart failure 2012: The Task Force for the Diagnosis and Treatment of Acute and Chronic Heart Failure 2012 of the European Society of Cardiology. Developed in collaboration with the Heart Failure Association (HFA) of the ESC. Eur Heart J 2012;33:1787-847.

33. Husain-Syed F, McCullough P, Birk HW, et al. Cardiopulmonary-renal interactions. A multidisciplinary approach. JACC 2015;65:2433-48. 


\title{
Arteriopatia obliterante periferica cronica degli arti inferiori
}

\author{
Francesco Dentali, ${ }^{1}$ Antonino Mazzone ${ }^{2}$ \\ ${ }^{1}$ Dipartimento di Medicina Clinica, Università dell'Insubria, Varese; ${ }^{2}$ Medicina Interna, Ospedale di Legnano (MI), Italia
}

\section{Introduzione}

Il termine arteriopatia obliterante periferica (AOP) degli arti inferiori si riferisce generalmente al restringimento o l'ostruzione di arterie provocati da un processo aterosclerotico in atto che riduce il flusso di sangue nelle gambe durante l'attività fisica $\mathrm{o}$ anche a riposo; è un marker di aterosclerosi sistemica e sta emergendo come un forte fattore di rischio per la morbilità e la mortalità cardiovascolare: infatti viene considerata un forte elemento predittivo di altri tipi di malattia cardiovascolare (CVD) [che comprendono la malattia coronarica (CAD) e/o la malattia cerebrovascolare] e di eventi cardiovascolari (CV), come l'infarto del miocardio (MI), gli accidenti cerebrovascolari e la morte. ${ }^{1}$ Una precoce diagnosi e una terapia medica aggressiva possono ridurre significativamente il profilo di rischio cardiovascolare in questi pazienti.

\section{Fattori di rischio}

I principali fattori di rischio associati all'aterosclerosi coronaria contribuiscono anche all'aterosclerosi del circolo periferico; quindi età, fumo di sigaretta, diabete mellito, ipertensione arteriosa, dislipidemia ed iperomocisteinemia aumentano il rischio di AOP.

\section{Età}

L'incidenza di AOP aumenta con l'età: si aggira allo $0,6 \%$ in soggetti di età compresa tra i $45-54$ anni, 2,5\% tra i 55-64 anni, 8,8\% tra i 65-74 anni; ${ }^{2}$ tende ad essere due volte maggiore nei maschi rispetto alle fem-

Corrispondente: Francesco Dentali, Dipartimento di Medicina Clinica, Università dell'Insubria, via Ravasi 2, 21100 Varese, Italia.

E-mail: fdentali@libero.it

Articolo pubblicato secondo la Creative Commons Attribution NonCommercial 3.0 License (CC BY-NC 3.0).

CCopyright F. Dentali e A. Mazzone, 2015

Licensee PAGEPress, Italy

QUADERNI - Italian Journal of Medicine 2015; 3:371-378 mine tra 50 e 70 anni, ma è quasi identica dopo i 70 anni. ${ }^{2-4}$ La maggioranza dei pazienti oltre i 60 anni $^{5} \mathrm{è}$ sintomatica.

\section{Diabete mellito}

Circa il 25\% dei pazienti di una determinata area geografica che si sottopongono a rivascolarizzazione degli arti inferiori sono diabetici, ${ }^{6-9} \mathrm{e}$ hanno un rischio 7 volte maggiore di amputazione degli arti rispetto ai non diabetici. ${ }^{10,11}$ Tuttavia, tale aumentato rischio ha un'origine probabilmente multifattoriale, dipendendo innanzitutto dal processo aterosclerotico più distale $\mathrm{e}$ generalizzato dei diabetici, ma anche dalla concomitante neuropatia sensoriale periferica che può favorire l'ulcerazione traumatica. ${ }^{12} \mathrm{Va}$ inoltre sottolineato che anche un'alterata tolleranza al glucosio è correlata a sviluppo di claudicatio intermittens (CI), con un rischio che aumenta di due volte negli uomini e di quattro nelle donne. ${ }^{13}$

\section{Fumo}

Il fumo è forse il fattore di rischio più importante per lo sviluppo di AOP, ancora più strettamente correlato a tale patologia che alla coronaropatia ${ }^{8}$. Tutti gli studi epidemiologici sulla AOP degli arti inferiori hanno confermato che il fumo contribuisce fortemente allo sviluppo di tale patologia ed alla sua progressione verso le manifestazioni più deleterie: $\mathrm{CI}$ invalidante, ischemia critica, amputazione). ${ }^{14,15} \mathrm{La}$ probabilità d'insorgenza di AOP è circa doppia nei fumatori rispetto ai non fumatori e la gravità della patologia è correlata alla quantità di sigarette fumate e alla durata del tempo in cui si è fumato. ${ }^{16} \mathrm{E}$ tre volte più probabile che i forti fumatori sviluppino CI rispetto ai non fumatori. ${ }^{17}$

\section{Ipertensione arteriosa}

L'ipertensione arteriosa è un fattore di rischio comune e importante di disordini vascolari, compresa 1'AOP. Tra i soggetti ipertesi alla prima visita, circa il $2-5 \%$ presenta CI, e tali percentuali tendono ad aumentare con l'età, mentre il $35-55 \%$ dei pazienti con AOP alla prima visita mostra anche di essere iperteso. ${ }^{18}$ In presenza di ipertensione e di AOP esiste un rischio notevolmente aumentato di infarto del miocardio e di ictus. 


\section{Iperlipidemia}

Circa il 50\% dei pazienti con AOP presenta iperlipidemia. Nello studio Framingham, una colesterolemia a digiuno $>270 \mathrm{mg} / \mathrm{dL}$ è stata associata ad un raddoppio dell'incidenza di CI. ${ }^{19}$ Anche se altri studi non hanno confermato la correlazione AOP/ipercolesterolemia, è stato tuttavia osservato che il trattamento dell'iperlipidemia riduce la progressione dell'aterosclerosi e l'incidenza della CI.

\section{Iperomocisteinemia}

L'iperomocisteinemia è un fattore di rischio di vasculopatia aterosclerotica per vasi periferici, coronarici e cerebrali, su cui si sta concentrando l'interesse degli studiosi. ${ }^{20-22}$ Tra l'altro, è stato osservato che il grado di progressione della CI è significativamente correlato ai livelli di omocisteina plasmatici. ${ }^{23}$

\section{Altri fattori di rischio}

Attualmente sono indagati altri potenziali fattori di rischio che si ritengono correlati, in vario modo e grado, alla patologia vascolare. Studi più recenti hanno identificato come la razza nera e l'insufficienza renale cronica possano essere considerati fattori di rischio associati all'insorgenza di AOP. ${ }^{24,25}$

Elevati markers di infiammazione inclusa la proteina $C$ reattiva ad alta sensibilità, l'interleuchina- 6 , il fibrinogeno, la $\beta-2$ microglobulina, la soluble vascular cell adhesion molecole-1, la soluble intercellular adhesion molecole- 1 e la cistatina $\mathrm{C}$ sono considerati nuovi fattori di rischio, la cui utilità clinica nel predire l'insorgenza e la prognosi di AOP stanno acquistando sempre più interesse. ${ }^{26-35}$ Una recente revisione della letteratura ha dimostrato come alcuni markers di stress ossidativo come il reactive ossigen species siano consistentemente aumentati nei pazienti con $\mathrm{AOP}^{36-38} \mathrm{e}$ in pazienti con CI..$^{39,40}$

Anche la concentrazione di markers di rimodellamento vascolare con come TGF-B1 e sRAGE sembra essere ridotta nei pazienti affetti da AOP. ${ }^{41,42}$ Questi biomarcatori possono essere utili non solo per determinare la presenza o assenza della patologia ma anche nel predire l'outcome e la prognosi del paziente affetto da AOP. Molti studi hanno riportato come livelli elevati di biomarkers di infiammazione (come IL-6, hsCRP, sVCAM1, sICAM-1) siano correlati ad uno stadio più severo di AOP e alle sue complicanze come l'amputazione e il rischio di infarto miocardico, stroke e morte. ${ }^{43-51}$

Alcuni studi hanno dimostrato come le concentrazioni plasmatiche della mieloperossidasi, della proteina galectina 3 e del NT-pro-BNP siano utili per eseguire una stratificazione del rischio di eventi avversi maggiori in pazienti con $\mathrm{AOP}^{52-55} \mathrm{e}$ suggeriscono il loro potenziale utilizzo come markers prognostici.
Comunque sono necessari ulteriori studi per confermare tale dati.

\section{Rischio di successivi eventi}

I dati dal registro REduction of Atherothrombosis for Continued Health (REACH) $)^{56}$ costituiscono un'interessante sorgente di informazioni sul rischio di successive eventi vascolari in pazienti con AOP. Esso ha raccolto 55.499 pazienti al baseline con malattia sintomatica, dei quali 39.675 erano eleggibili per un follow-up di 3 anni.

Di questi, al baseline, 6,3\% (2485) avevano solo AOP; 5,7\% (2271) avevano AOP più CAD; $1,5 \%$ (614) avevano $\mathrm{AOP}$ più malattia cerebrovascolare e 1,9\% (748) avevano $\mathrm{AOP}$ più $\mathrm{CAD}$ più malattia cerebrovascolare.

I pazienti arruolati nel trial con AOP hanno mostrato il più alto tasso di eventi a 3 anni di MI, stroke/morte/ospedalizzazione (40,4\%). Anche il tasso di re-ricovero in ospedale per ogni evento cardiovascolare diverso da MI/stroke era più alto per la popolazione con AOP $(33,6 \%)$, in confronto al $23 \%$ di quelli con CAD e al $18,7 \%$ dei pazienti con malattia cerebrovascolare.

In un periodo di 3 anni, i pazienti con sola AOP al baseline avevano una più alta probabilità di progredire verso il coinvolgimento di altri distretti vascolari: quasi il $10 \%$ progrediva verso una malattia polivascolare, circa il $4 \%$ verso una CAD o una malattia cerebrovascolare. La prevalenza totale di AOP, basata su test non invasivi, era dell' $11,7 \%$, ed aumentava con l'età e con i livelli dei lipidi. Nonostante le forti raccomandazioni delle linee guida, si considera che l'AOP sia ancora sottotrattata in molti paesi. ${ }^{57}$

\section{Quali sono i metodi di valutazione dei rischi e stratificazione prognostica (anche rispetto alle comorbilità esistenti)?}

La diagnosi di AOP inizia dalla storia clinica dei pazienti, dalla loro sintomatologia e dal loro stato di salute generale, dal fatto che fumino o no e dall'eventuale presenza di ipertensione arteriosa e di diabete. L'esame fisico può rivelare estremità fredde e altri segni di malattia delle arterie periferiche subclinica. Sono importanti la presenza di soffi vascolari e l'ipoasfigmia (non dimenticare di controllare anche dietro le ginocchia e i piedi).

\section{Classificazione dell'arteriopatia obliterante periferica: strumenti clinici}

Sono state elaborate delle scale nella valutazione dell'AOP per consentire ai medici di descrivere la condizione clinica dei loro pazienti, utilizzando terminologie e norme comuni per i reports. ${ }^{58,59}$ Sia la 
classificazione di Rutherford che quella di Fontaine non vengono utilizzati di routine nella pratica clinica, ma soprattutto in contesti di ricerca. Tuttavia, come le linee guida dell'American College of Cardiology/American Heart Association (ACC/AHA) 2005 hanno sottolineato, possono essere utili per la comunicazione standardizzata tra i $\operatorname{clinici}^{60}$ (Tabella 1).

\section{Ankle-brachial index negli adulti per la valutazione dell'arteriopatia obliterante periferica}

L'ankle-brachial index (ABI, indice caviglia braccio) è uno dei più comuni test utilizzati per diagnosticare l'AOP: tale indice rappresenta il rapporto tra le pressioni sistoliche misurate alla caviglia e all'arteria brachiale. Si posiziona un manicotto pneumatico attorno alla caviglia, lo si gonfia fino a una pressione sovrasistolica e successivamente lo si sgonfia, mentre si rileva, tramite una sonda Doppler posizionata sulle arterie pedidia e tibiale posteriore, la ricomparsa di flusso, rilevando così la pressione sistolica alla caviglia (scegliendo il valore più alto). La pressione sistolica brachiale può essere rilevata in modo routinario utilizzando uno stetoscopio per ascoltare il primo suono di Korotkoff.

Secondo le 2011 ACCF/AHA Focused Update Recommendations l'ABI a riposo deve essere utilizzato per stabilire la diagnosi di AOP in pazienti sospetti, definiti come soggetti con uno o più dei seguenti elementi: dolori da sforzo alle gambe, ferite che non si rimarginano, età di 65 anni e più, o 50 anni di età con una storia di fumo o di diabete. I risultati dell'ABI dovrebbero essere riportati in modo uniforme con valori non comprimibili definiti come maggiori di 1,40, valori normali da 1,00 a 1,40 , borderline da 0,91 a 0,99 , e anormali 0,90 o meno. ${ }^{61}$ Tra i pazienti con bassi valori di $\mathrm{ABI}(\leq 0,90)$ rilevati sia in coorti basate sulla popolazione che in quelle basate sull' assistenza primaria ad alto rischio, solo il $10-15 \%$ ha claudicatio intermittens. ${ }^{62,63}$ Inoltre, la USPSTF (US Preventive Services Task Force) ha concluso che non c'è sufficiente evidenza per definire il bilancio rischio/beneficio di uno screening per la valutazione tra $\mathrm{AOP}$ e il rischio di CVD con l'ABI. $L a$ USPSTF non ha trovato alcuna prova che lo screening e il trattamento dell' $A O P$ in pazienti asintomatici portI a benefici clinicamente importanti. Essa ha inoltre esaminato i potenziali benefici di aggiungere l'ABI al FRS (Framingham risk score) evidenziando che ciò si traduce in alcune riclassificazioni del rischio del paziente; tuttavia, quanto spesso la riclassificazione sia appropriata o se si traduca in un miglioramento dei risultati clinici non è noto. ${ }^{64}$

\section{Quali sono le azioni da realizzare, su misura per il singolo paziente?}

Poiché l'AOP è una manifestazione di aterosclerosi sistemica nelle estremità inferiori, i suoi obiettivi terapeutici farmacologici sono quasi identici a quelli della malattia cerebrovascolare e della CVD. Smettere di fumare, l'esercizio fisico, e un aggressiva modificazione farmacologica dei fattori rischio $\mathrm{CV}$, tra cui il diabete, l'ipertensione, le dislipidemie, e la terapia antiaggregante per tutta la vita, rappresentano i capisaldi del trattamento. Per ulteriori dettagli su ciascuna malattie ci riferiamo alle corrispondenti sezioni di questo documento.

\section{Il ruolo dell'esercizio nell'arteriopatia obliterante periferica}

L'esercizio fisico, combinato con la prevenzione secondaria globale, è molto utile per i pazienti con

Tabella 1. Classificazioni di Lériche-Fontaine e Rutherford.

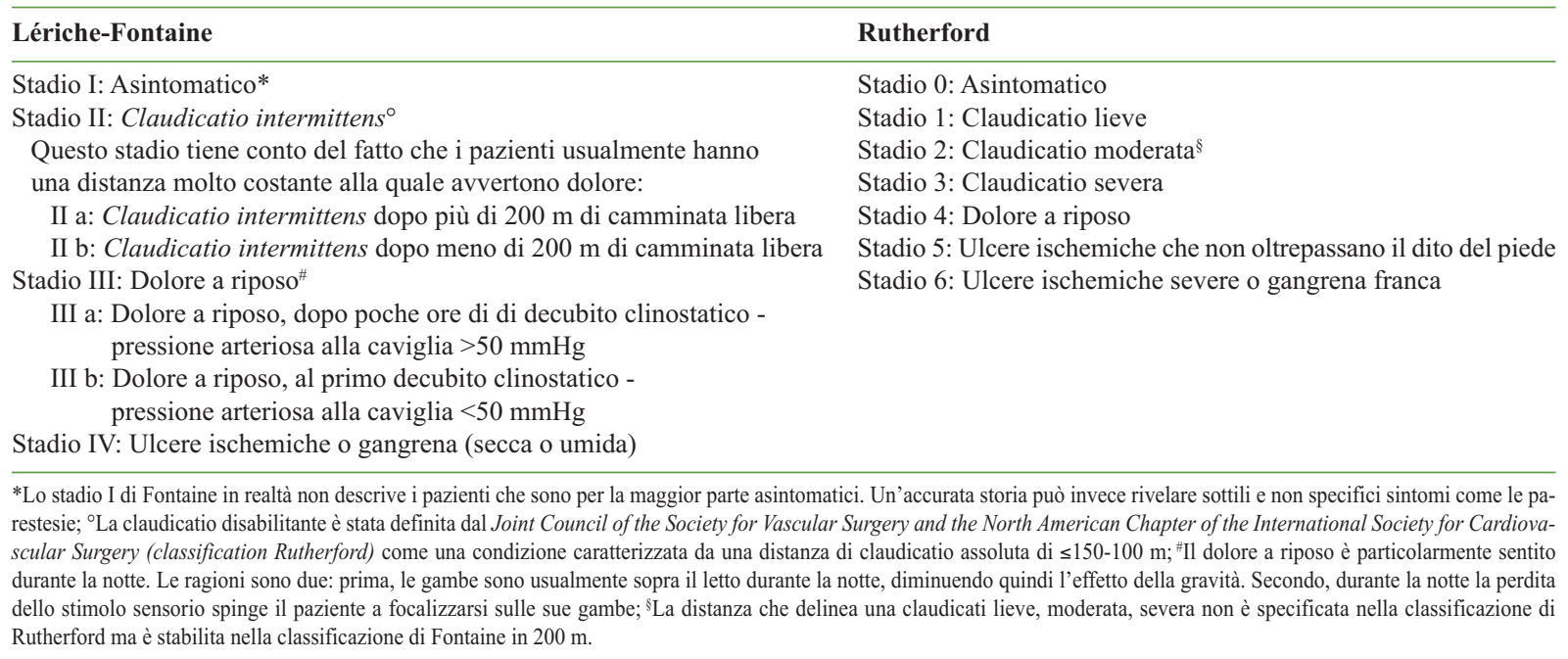


AOP, conservando o migliorando la capacità funzionale e riducendo gli eventi cardiovascolari. Gli effetti dell'esercizio includono alcuni meccanismi potenziali, quali l'aumento del flusso collaterale, una migliore vasodilatazione NO-dipendente ed energetica mitocondriale, una riduzione dell'infiammazione sistemica. ${ }^{65}$

\section{Terapia antitrombotica}

Secondo le linee guida ACCP 2012 per pazienti la prevenzione secondaria in pazienti con AOP sintomatica, viene raccomandato uno dei due seguenti regimi antitrombotici da continuare a lungo termine: aspirina 75-100 $\mathrm{mg}$ al giorno o clopidogrel $75 \mathrm{mg}$ al giorno (entrambe raccomandazioni grado $1 \mathrm{~A}$ ). Si consiglia di non utilizzare la terapia antiaggregante con aspirina più clopidogrel (grado 2B). Per i pazienti con CI refrattaria si consiglia anzitutto l'esercizio fisico e la cessazione del fumo; come farmaco, si può utilizzare il cilostazolo, mentre non è consigliabile l'uso di pentossifillina, eparinoidi o prostanoidi (grado $2 \mathrm{C}$ ). I prostanoidi sono raccomandati nei pazienti con AOP sintomatica e ischemia critica degli arti e/o dolore a riposo che non sono candidati per un intervento vascolare.

Per i pazienti sottoposti a percutaneous transluminal angioplasty (PTA) delle arterie periferiche con o senza stent, viene raccomandato un trattamento a lungo termine con aspirina $(75-100 \mathrm{mg} / \mathrm{die})$ o clopidogrel (75 mg/die): grado $1 \mathrm{~A}$. Per i pazienti sottoposti a PTA periferica dell'arteria con impianto di stent, viene suggerita una terapia con un singolo antiaggregante piuttosto che doppio. ${ }^{66}$ Nonostante queste raccomandazioni, l'utilità dell'aspirina nel prevenire l'AOP non è così certa. ${ }^{67}$ Una metanalisi del Antithrombotic trialists' collaboration non dimostra benefici dell'aspirina nel ridurre gli eventi CV nell'AOP. ${ }^{68}$

L'effetto dell'aspirina sugli eventi cardiovascolari nei pazienti con AOP preclinica è stato studiato nel trial aspirin for asymptomatic atherosclerposis con una randomizzazione a doppio cieco di 3350 soggetti con un ABI basso $(\leq 0,95)$ e un follow-up per 8,2 anni. Non è stata riscontrata una differenza statisticamente significativa negli endpoint tra i soggetti trattati con aspirina $(100 \mathrm{mg})$ e il gruppo placebo. ${ }^{69}$

Alcuni nuovi antiaggreganti come il prasugrel, il ticagrelor e la picotamide sembrano più efficaci dell'aspirina nei pazienti con AOP, particolarmente se diabetici. Un nuovo antagonista del protease-activated receptor -1 , il vorapaxar, riduce significativamente i tassi di ospedalizzazione per l'ischemia acuta della gamba e la rivascolarizzazione arteriosa periferica. ${ }^{70}$

Non vi sono evidenza che la terapia con warfarin riduca l'incidenza di eventi avversi correlati alla sola AOP.

\section{Statine}

L'ipercolesterolemia, che si riscontra dal $45 \%$ al $59 \%$ dei pazienti AOP sintomatici, è un fattore di rischio rilevante per l'AOP. Un beneficio del trattamento con statine sugli eventi cardiovascolari e cerebrovascolari è stato dimostrato nei pazienti con AOP e ipercolestero-lemia, nei quali gli effetti aggiuntivi delle statine, come il miglioramento della distanza percorsa a piedi senza dolore (pain-free walking distance) e la qualità della vita sono stati confermati. $^{71}$

\section{Terapia dietetica}

Alcuni studi hanno dimostrato come un aumentato apporto con la dieta di folati e vitamina $\mathrm{D}$ possono proteggere contro l'insorgenza di AOP. Numerosi studi hanno evidenziato come la prevalenza di AOP sia più alta in coloro che assumono una dieta ricca in grassi saturi, carne e derivati rispetto a coloro che ne fanno un uso più limitato. ${ }^{72-74} \mathrm{Al}$ contrario, coloro che assumevano maggiori quantità di cibi che erano ricchi di grassi poli-insaturi, di fibre e antiossidanti (vitamine $\mathrm{A}, \mathrm{C}$ e E) avevano un rischio significativamente più basso di sviluppare AOP rispetto a coloro che consumavano minori quantità di tali alimenti. . $^{70,71,75-78}$

Una dieta mediterranea ha un effetto protettivo simile contro gli outcomes cardio-vascolari in pazienti con AOP e in pazienti con altre patologie cardio-vascolari. ${ }^{79,80}$

Gli acidi grassi essenziali omega-3 potrebbero avere un effetto anti-infiammatorio e potrebbero avere un effetto protettivo contro il danno endoteliale e l'aterosclerosi. ${ }^{81}$ Comunque la maggior parte degli studi non ha mostrato alcun effetto con la supplementazione di acidi grassi essenziali omega-3 sui sintomi e la progressione della AOP. Una meta-analisi di 10 studi ha dimostrato che la supplementazione con acidi grassi essenziali omega 3 riduce significativamente le misure di rigidità arteriosa (compliance arteriosa, la cardioankel pulse wave velocity (PVW) e brachial-ankle PWV in varie corti. ${ }^{82} \mathrm{E}$ in corso uno studio che sta valutando l'effetto della supplementazione per un anno con acidi grassi essenziali omega-3 in pazienti con AOP e si pensa che si possano evidenziare gli stessi effetti sulla rigidità arteriosa di tali pazienti. ${ }^{83}$

\section{Qualità delle cure nell'arteriopatia obliterante periferica}

La lista della dichiarazione di qualità sviluppata per l'AOP nel 2014 dal National Institute for Health and Care Excellence (NICE) viene riportato nella Tabella $2 .{ }^{84}$ 
Tabella 2. L'elenco degli statements di qualità del National Institute for Health and Care Excellence (NICE) per l'arteriopatia obliterante periferica.

Statement 1

I pazienti con sintomi (o che sono a rischio di svilupparli) di AOP vanno considerati per una valutazione clinica e un ABI

Statement 2

I pazienti con AOP vanno considerati per una valutazione di altre morbilità cardiovascolari e di fattori di rischio modificabili

Statement 3

I pazienti con claudicatio intermittens vanno considerati per un programma di esercizio sotto stretto controllo

Statement 4

I pazienti con AOP candidati alla rivascolarizzazione che necessitano di ulteriori immagini dopo un un'ecografia vanno sottoposti ad angiorisonanza magnetica

\section{Statement 5}

I pazienti con claudicatio intermittens vanno inviati all'angioplastica solo quando le immagini hanno confermato che ciò è appropriato, dopo aver informato i pazienti stessi dei benefici delle modificazioni dei fattori di rischio, e dopo che un intenso programma di riabilitazione fisica non ha migliorato i sintomi

AOP, arteriopatia obliterante periferica; $\mathrm{ABI}$, ankle-brachial index.

\section{Bibliografia}

1. Lin S, Olson C, Johnson E, et al. Screening for peripheral artery disease with ankle brachial index testing: a systematic evidence review for the U.S. Preventive Services Task Force. Evidence Synthesis No. 100. AHRQ Publication No. 12-05162- F-1. Rockville, MD: Agency for Healthcare Research and Quality; 2013.

2. Stoffers HE, Kaiser V, Knottnerus JA. Prevalence in general practice. In: Fowkes FGR, ed. Epidemiology of peripheraI vascular disease. London: Springer-Verlag; 1991. pp 109-115.

3. Cary NRB. Pathology of peripheral arterial disease. In: Tooke JE, Lowe GDO, eds. Texbook of vascular medicine. London: Arnold; 1996. pp 143-148.

4. Vogt MT, Wolfson SK, Kuller LH. Lower extremity arterial disease and the aging process: a review. J Clin Epidemiol 1992;45:529-42.

5. Dormandy JA, Ray S. The natural history of peripheral arterial disease. In: Tooke JE, Lowe GDO, eds. Textbook of vascular medicine. London: Arnold; 1996. pp 162-175.

6. Widmer LK, Biland L, Da Silva A. Risk profile and occlusive periphery artery disease (OPAD). In: Proc. 13th International Congress of Angiology, 9-14 June 1985, Athens, Greece, p 28.

7. Stout RW. Diabetes, atherosclerosis and aging. Diabetes Care 1990;13:20-3.

8. Gordon T, Kannel WB. Predisposition to atherosclerosis in the head, heart, and legs. The Framingham study. JAMA 1972;221:661-6.

9. Farkouh ME, Rihal CS, Gersh BJ, et al. Influence of coronary heart disease on morbidity and mortality after lower extremity revascularization surgery: a populationbased study in Olmsted County, Minnesota (1970-1987). J Am Coll Cardiol 1994;24:1290-6.

10. Jonason T, Ringqvist I. Factors of prognostic importance for subsequent rest pain in patients with intermittent claudication. Acta Med Scand 1985;218:27-33.

11. Hughson WG, Mann JI, Garrod A. Intermittent claudication: prevalence and risk factors. BMJ 1978;1:1379-81.
12. Weitz JI, Byrne J, Clagett GP, et al. Diagnosis and treatment of chronic arterial insufficiency of the lower extremities: a critical review. Circulation 1996;94: 3026-49.

13. Criqui MH, Browner D, Fronek A, et al. Peripheral arterial disease in large vessels is epidemiologically distinct from small vessel disease. An analysis of risk factors. Am J Epidemiol 1989;129:1110-9.

14. Farkouh ME, Rihal CS, Gersh BJ, et al. Influence of coronary heart disease on morbidity and mortality after lower extremity revascularization surgery: a populationbased study in Olmsted County, Minnesota (1970-1987). J Am Coll Cardiol 1994;24:1290-6.

15. Schroll M, Munck O. Estimation of peripheral arteriosclerotic disease by ankle blood pressure measurements in a population study of 60 -year-old men and women. J Chronic Dis 1981;34:261-9.

16. [No authors listed]. Lifestyle measures to tackle atherosclerotic disease. DTB 2001;39:21-4.

17. Powell JT. Smoking. In: Fowkes FGR, ed. Epidemiology of peripheraI vascular disease. London: Springer; 1991. pp 141-154.

18. Makin A, Lip GY, Silverman S, Beevers DG. Peripheral vascular disease and hypertension: A forgotten association? J Hum Hypertens 2001;15:447-54.

19. Kannel WB, Skinner JJ Jr, Schwartz MJ, Shurtleff D. Intermittent claudication. Incidence in the Framingham study. Circulation 1970;41:875-83.

20. Criqui MH. Systemic atherosclerosis risk and the mandate for intervention in atherosclerotic peripheral arterial disease. Am J Cardiol 2001;88:43J-7J.

21. Clarke R, Daly L, Robinson K, et al. Hyperhomocysteinemia: an independent risk factor for vascular disease. N Engl J Med 1991;324:1149-55.

22. Mölgaard J, Malinow MR, Lassvik C, et al. Hyperhomocyst(e)inaemia: an independent risk factor for intermittent claudication. J Intern Med 1992;231:273-9.

23. Taylor LM Jr, DeFrang RD, Harris EJ Jr, Porter JM. The association of elevated plasmahomocyst(e)ine with progression of symptomatic peripheral arterial disease. J Vasc Surg 1991;13:128-36. 
24. Selvin E, Erlinger TP. Prevalence of and risk factors for peripheral arterial disease in the United States: results from the National Health and Nutrition Examination Survey, 1999-2000. Circulation 2004;110:738-43.

25. O'Hare AM, Vittinghoff E, Hsia J, Shlipak MG. Renal insufficiency and the risk of lower extremity peripheral arterial disease: results from the heart and estrogen/progestin replacement study (HERS). J Am Soc Nephrol 2004;15:1046-51.

26. Ridker P, Stampfer MJ, Rifai N. Novel risk factors for atherosclerosis: a comparison of C-reactive protein, fibrinogen, homocysteine, lipoprotein (a), and standard cholesterol screening as predictors of peripheral arterial disease. JAMA 2001;285:2481-5.

27. Wilson AM, Shin DS, Weatherby C, et al. Asymmetric dimethylarginine correlates with measures of disease severity, major adverse cardiovascular events and allcause mortality in patients with peripheral arterial disease. Vasc Med 2010;15:267-74.

28. Wilson AM, Kimura E, Harada RK, et al. B2-microglobulin as a biomarker in peripheral arterial disease: proteomic profiling and clinical studies. Circulation 2007;116:1396-403.

29. Hiatt WR, Zakharyan A, Fung ET, et al. A validated biomarker panel to identify peripheral artery disease. Vasc Med 2012;17:386-93.

30. Joosten MM, Pai JK, Bertoia ML, et al. b2-microglobulin, cystatin C, and creatinine and risk of symptomatic peripheral artery disease. J Am Heart Assoc 2014;3: e000803.

31. Joosten MM, Pai JK, Bertoia ML, et al. $\beta 2$-microglobulin, cystatin $\mathrm{C}$, and creatinine and risk of symptomatic peripheral artery disease. J Am Heart Assoc 2014;3: e000803.

32. Cheng CH, Chen YS, Shu KH, et al. Higher serum levels of soluble intracellular cell adhesion molecule-1 and soluble vascular cell adhesion molecule predict peripheral artery disease in haemodialysis patients. Nephrology (Carlton) 2012;17:718-24.

33. Gardner AW, Parker DE, Montgomery PS, et al. Impaired vascular endothelial growth factor a and inflammation in patients with peripheral artery disease. Angiology 2014;65:683-90.

34. Pradhan AD, Rifai N, Ridker PM. Soluble intercellular adhesion molecule-1, soluble vascular adhesion molecule-1, and the development of symptomatic peripheral arterial disease in men. Circulation 2002;106:820-5.

35. McDermott MM, Liu K, Ferrucci L, et al. Relation of interleukin-6 and vascular cellular adhesion molecule1 levels to functional decline in patients with lower extremity peripheral arterial disease. Am J Cardiol 2011;107:1392-8.

36. Pipinos II, Judge AR, Zhu Z, et al. Mitochondrial defects and oxidative damage in patients with peripheral arterial disease. Free Radic Biol Med 2006;41:262-9.

37. Belch JJ, Mackay IR, Hill A, et al. Oxidative stress is present in atherosclerotic peripheral arterial disease and further increased by diabetes mellitus. Int Angiol 1995; 14:385-8.

38. Kals J, Kampus P, Kals M, et al. Inflammation and oxi- dative stress are associated differently with endothelial function and arterial stiffness in healthy subjects and in patients with atherosclerosis. Scan J Clin Lab Investig 2008;68:594-601.

39. Hickman P, Harrison DK, Hill A, et al. Exercise in patients with intermittent claudication results in the generation of oxygen derived free radicals and endothelial damage. Adv Exp Med Biol 1994;361:565-70.

40. Silvestro A, Scopacasa F, Oliva G, et al. Vitamin C prevents endothelial dysfunction induced by acute exercise in patients with intermittent claudication. Atherosclerosis 2002;165:277-83.

41. Signorelli SS, Anzaldi M, Fiore V, et al. Patients with unrecognized peripheral arterial disease (PAD) assessed by ankle-brachial index $(\mathrm{ABI})$ present a defined profile of proinflammatory markers compared to healthy subjects. Cytokine 2012;59:294-8.

42. Catalano M, Cortelazzo A, Santi R, et al. The Pro12Ala polymorphism of peroxisome proliferator-activated receptor- $\gamma 2$ gene is associated with plasma levels of soluble rage (receptor for advanced glycation endproducts) and the presence of peripheral arterial disease. Clin Biochem 2008;41:981-5.

43. Signorelli SS, Anzaldi M, Fiore V, et al. Patients with unrecognized peripheral arterial disease (PAD) assessed by ankle-brachial index (ABI) present a defined profile of proinflammatory markers compared to healthy subjects. Cytokine 2012;59:294-8.

44. Catalano M, Cortelazzo A, Santi R, et al. The Pro12Ala polymorphism of peroxisome proliferator-activated receptor- $\gamma 2$ gene is associated with plasma levels of soluble rage (receptor for advanced glycation endproducts) and the presence of peripheral arterial disease. Clin Biochem 2008;41:981-5.

45. Wildman RP, Muntner P, Chen J, et al. Relation of inflammation to peripheral arterial disease in the national health and nutrition examination survey, 1999-2002. Am J Cardiol 2005;96:1579-83.

46. De Haro Miralles J, Martinez-Aguilar E, Florez A, et al. Nitric oxide: Link between endothelial dysfunction and inflammation in patients with peripheral arterial disease of the lower limbs. Interact Cardiovasc Thorac Surg 2009;9:107-12.

47. Murabito JM, Keyes MJ, Guo CY, et al. Cross-sectional relations of multiple inflammatory biomarkers to peripheral arterial disease: the Framingham offspring study. Atherosclerosis 2009;203:509-14.

48. McDermott MM, Guralnik JM, Corsi A, et al. Patterns of inflammation associated with peripheral arterial disease: the inchianti study. Am Heart J 2005;150:276-81.

49. Cassar K, Bachoo P, Ford I, et al. Markers of coagulation activation, endothelial stimulation and inflammation in patients with peripheral arterial disease. Eur J Vasc Endovasc Surg 2005;29:171-6.

50. Owens CD, Ridker PM, Belkin M, et al. Elevated Creactive protein levels are associated with postoperative events in patients undergoing lower extremity vein bypass surgery. J Vasc Surg 2007;45:2-9; discussion 9.

51. McDermott MM, Liu K, Ferrucci L, et al. Circulating blood markers and functional impairment in peripheral 
arterial disease. J Am Geriatr Soc 2008;56:1504-10.

52. Fan J, Jouni H, Khaleghi M, et al. Serum N-terminal pro-B-type natriuretic peptide levels are associated with functional capacity in patients with peripheral arterial disease. Angiology 2012;63:435-42.

53. Hewing B, Parathath S, Barrett T, et al. Effects of native and myeloperoxidase-modified apolipoprotein A-I on reverse cholesterol transport and atherosclerosis in mice. Arterioscler Thromb Vasc Biol 2014;34:779-89.

54. Liu C, Desikan R, Ying Z, et al. Effects of a novel pharmacologic inhibitor of myeloperoxidase in a mouse atherosclerosis model. PLoS One 2012;7:50767.

55. Van Kimmenade RR, Januzzi JL Jr. Emerging biomarkers in heart failure. Clin Chem 2012;58:127-38.

56. Alberts M, Bhatt D, Mas J, et al. Three-year follow-up and event rates in the international reduction of Atherothrombosis for Continued Health Registry. Eur Heart J 2009;30:2318-26.

57. Müller-Bühl U, Laux G, Szecsenyi J. Secondary pharmacotherapeutic prevention among german primary care patients with peripheral arterial disease. Int J Vasc Med 2011;2011:316496.

58. Becker F. La classification de l'insuffisance artérielle des membres inférieurs de Leriche et Fontaine: une réactualisation nécessaire. Méd Hyg 1991;49:143-9.

59. Rutherford RB, Baker JD, Ernst C, et al. Recommended standards for reports dealing with lower extremity ischemia: revised version. J Vasc Surg 1997;26:517-38.

60. Hirsch AT, Haskal ZJ, Hertzer NR, et al, ACC/AHA 2005 Practice Guidelines for the management of patients with peripheral arterial disease (lower extremity, renal, mesenteric, and abdominal aortic): a collaborative report from the American Association for Vascular Surgery/Society for Vascular Surgery, Society for Cardiovascular Angiography and Interventions, Society for Vascular Medicine and Biology, Society of Interventional Radiology, and the ACC/AHA Task Force on Practice Guidelines (Writing Committee to Develop Guidelines for the Management of Patients With Peripheral Arterial Disease): endorsed by the American Association of Cardiovascular and Pulmonary Rehabilitation; National Heart, Lung, and Blood Institute; Society for Vascular Nursing; TransAtlantic Inter-Society Consensus; and Vascular Disease Foundation. Circulation 2006;113: e463-654.

61. Rooke TW, Hirsch AT, Misra S, et al. 2011 ACCF/AHA focused update of the guideline for the management of patients with peripheral artery disease (updating the 2005 guideline): a report of the American College of Cardiology Foundation/American Heart Association Task Force on Practice Guidelines. Circulation 2011; 124:2020-45.

62. Feigelson HS, Criqui MH, Fronek A, et al. Screening for peripheral arterial disease: the sensitivity, specificity, and predictive value of noninvasive tests in a defined population. Am J Epidemiol 1994;140:526-34.

63. Hirsch AT, Criqui MH, Treat-Jacobson D, et al. Peripheral arterial disease detection, awareness, and treatment in primary care. JAMA 2001;286:1317-24.

64. Moyer VA; U.S. Preventive Services Task Force. Scree- ning for peripheral artery disease and cardiovascular disease risk assessment with the ankle-brachial index in adults: U.S. Preventive Services Task Force Recommendation Statement. Ann Intern Med 2013;159:342-8.

65. Hamburg NM, Baldy GJ. Exercise rehabilitation in peripheral artery disease: functional impact and mechanisms of benefits. Circulation 2014;123:87-97.

66. Alonso-Coello P, Bellmunt S, McGorrian C, et al. American College of Chest Physicians, antithrombotic therapy in peripheral artery disease: antithrombotic therapy and prevention of thrombosis, 9th ed: American College of Chest Physicians Evidence-Based Clinical Practice Guidelines. Chest 2012;141:e669S-90S.

67. Poredos P, Jezovnik MK. Do the effects of secondary prevention of cardiovascular events in PAD patients differ from other atherosclerotic disease? Int J Mol Sci 2015; 16:14477-89.

68. Baigent C, Blackwell L, Collins R, et al. Aspirin in the primary and secondary prevention of vascular disease: Collaborative meta-analysis of individual participant data from randomised trials. Lancet 2009;373:849-1860.

69. Fowkes FG, Price JF, Stewart MC, et al. Aspirin for prevention of cardiovascular events in a general population screened for a low ankle brachial index: a randomized controlled trial. JAMA 2010;303:841-8.

70. Bonaca MP, Scirica BM, Creager MA, et al. Vorapaxar in patients with peripheral artery disease results from TRA2 ${ }^{\circ}$ P-TIMI 50. Circulation 2013;127:1522-9.

71. Heart Protection Study Collaborative Group. MRC/BHF heart protection study of cholesterol lowering with simvastatin in 20,536 high-risk individuals: a randomised placebo-controlled trial. Lancet 2002;360:7-22.

72. Donnan PT, Thomson M, Fowkes FG, et al. Diet as a risk factor for peripheral arterial disease in the general population: the Edinburgh Artery Study. Am J Clin Nutr 1993;57:917-21.

73. Gimeno SG, Hirai AT, Harima HA, et al. Fat and fiber consumption are associated with peripheral arterial disease in a cross-sectional study of a Japanese-Brazilian population. Circ J 2008;72:44-50.

74. Katsouyanni K, Skalkidis Y, Petridou E, et al. Diet and peripheral arterial occlusive disease: the role of poly-, mono-, and saturated fatty acids. Am J Epidemiol 1991;133:24-31.

75. Antonelli-Incalzi R, Pedone C, McDermott MM, et al. Association between nutrient intake and peripheral artery disease: results from the InCHIANTI study. Atherosclerosis 2006;186:200-6.

76. Lane JS, Magno CP, Lane KT, et al. Nutrition impacts the prevalence of peripheral arterial disease in the United States. J Vasc Surg 2008;48:897-904.

77. Leng GC, Horrobin DF, Fowkes FG, et al. Plasma essential fatty acids, cigarette smoking, and dietary antioxidants in peripheral arterial disease. A population-based casecontrol study. Arterioscler Thromb 1994;14:471-8.

78. Tornwall ME, Virtamo J, Haukka JK, et al. Prospective study of diet, lifestyle, and intermittent claudication in male smokers. Am J Epidemiol 2000;151:892-901.

79. De Lorgeril M, Salen P. Mediterranean diet in secondary prevention of CHD. Public Health Nutr 2011;14:2333-7. 
80. Hardin-Fanning F. The effects of a Mediterranean-style dietary pattern on cardiovascular disease risk. Nurs Clin North Am 2008;43:105-15.

81. Holy EW, Forestier M, Richter EK, et al. Dietary $\alpha$-linolenic acid inhibits arterial thrombus formation, tissue factor expression, and platelet activation. Arterioscler Thromb Vasc Biol 2011;31:1772-80.

82. Pase MP, Grima NA, Sarris J. Do long-chain n-3 fatty acids reduce arterial stiffness? A meta-analysis of randomised controlled trials. Br J Nutr 2011;106:974-80.
83. Leyva DR, Zahradka P, Ramjiawan B, et al. The effect of dietary flaxseed on improving symptoms of cardiovascular disease in patients with peripheral artery disease. Rationale and design of the FLAX-PAD randomized controlled trial. Contemp Clin Trials 2011;32:724-30.

84. National Institute for Health and Care Excellence (NICE). Peripheral arterial disease. Issued: January 2014. NICE quality standard 52. Available from: http://www.nice.org.uk/guidance/qs52/resources/guidance-peripheral-arterial-disease-pdf 


\title{
La prevenzione cardiovascolare secondaria nel paziente con diabete mellito
}

\author{
Giuseppe Augello, ${ }^{1}$ Luigi Magnani ${ }^{2}$ \\ ${ }^{1}$ UOC Medicina Interna, Ospedale di Canicattì, ASP 1 Agrigento; ${ }^{2}$ UOC Medicina Interna, Ospedale di Voghera, AO Provincia \\ di Pavia, Italia
}

\section{Introduzione}

Le malattie cardiovascolari sono la causa principale di mortalità e di morbilità dei soggetti affetti da diabete tipo 2, per i quali la spettanza di vita, rispetto ai soggetti non diabetici, si riduce di 10-15 anni a partire dai 50 anni. ${ }^{1,2}$

In Italia la popolazione diabetica presenta un eccesso di mortalità pari al $30-40 \%$ rispetto alla popolazione non diabetica e ogni anno si registrano più di 70.000 ricoveri per diabete principalmente dovuti a complicanze cardiovascolari quali infarto del miocardio, ictus cerebrale, insufficienza renale e amputazioni degli arti inferiori. ${ }^{3-6}$

Ne consegue che il consumo di risorse sanitarie per le persone con diabete è 2,5 volte maggiore rispetto a quello per le persone non diabetiche di pari età e genere.

Nel nostro Paese la spesa totale stimata dall'International Diabetes Federation nel 2010 è stata di 11 milioni di dollari con una previsione di crescita entro il 2030 di oltre il 14\%: attualmente il diabete occupa il secondo posto tra le patologie per i più alti costi diretti. In assenza di complicanze, questi sono pari a circa 800 euro/anno a persona, mentre in presenza di complicanze possono variare tra i 3000 e i 36.000 euro/anno a persona.

L'impatto socioeconomico del diabete si avvia, quindi, a essere sempre più difficile da sostenere per la comunità, in assenza di un'efficace prevenzione primaria e secondaria, ${ }^{7-9}$ ed è necessario che il diabete mellito tipo 2 venga considerato uno stato di malattia cardiovascolare accelerato, associato ad iperglicemia

Corrispondente: Giuseppe Augello, UOC Medicina Interna, Ospedale di Canicattì, ASP 1 Agrigento, Italia.

E-mail: dr.augello@alice.it

Articolo pubblicato secondo la Creative Commons Attribution NonCommercial 3.0 License (CC BY-NC 3.0).

CCopyright G. Augello e L. Magnani, 2015

Licensee PAGEPress, Italy

QUADERNI - Italian Journal of Medicine 2015; 3:379-389 perché è ormai chiaro che la riduzione del rischio cardiovascolare deve avere la precedenza sulla riduzione della glicemia.

\section{Studi clinici ed evidenze}

Lo storico studio UKPDS (United Kingdom prospective diabetes study), ${ }^{10}$ terminato nel 1997 si era concluso con l'evidenza che una terapia intensiva fosse in grado di ritardare l'insorgenza e ridurre la progressione dell'albuminuria e della retinopatia, ma non di diminuire il rischio di infarto miocardico (IMA); risultati, questi, simili a quelli evidenziati dal DCCT (diabetes control and complications trial). ${ }^{11}$ Tali risultati si sono mantenuti negli anni, anche quando è cessato il controllo intensivo del diabete e i valori di $\mathrm{HbA1c}$ sono diventati sovrapponibili tra il gruppo trattato più intensamente e quello trattato meno intensamente. ${ }^{12}$ Per spiegare questo fenomeno è stato invocato il cosiddetto effetto eredità o meglio memoria metabolica: nel diabetico di nuova diagnosi il processo ateromasico è ancora in una fase precoce per cui un buon controllo glicemico eseguito precocemente comporta una ridotta esposizione ai prodotti della glicosilazione, influenzando per decenni, con un trend positivo, il decorso della malattia aterosclerotica. ${ }^{13}$ Visti questi presupposti, si è sempre più convinti che bisogna agire sulla memoria metabolica non solo con un trattamento aggressivo precoce dell'iperglicemia, ma anche con il contemporaneo utilizzo di composti efficaci sulla formazione degli AGE (prodotti finali avanzati della glicosilazione) e sui prodotti reattivi a livello mitocondriale che sono direttamente implicati nello sviluppo delle complicanze vascolari della malattia diabetica. Questa potrebbe essere, in un prossimo futuro, una strategia vincente per la prevenzione delle complicanze del diabete. ${ }^{14}$

Bisogna però ricordare che risultati dell'UKPDS sono apparentemente in contrasto con quelli dei più recenti studi $\mathrm{ACCORD}^{15}$ (action to control cardiovascular risk in diabetes), ADVANCE (action in diabetes and vascular disease: preterax and diamicron $\mathrm{mr}$ controlled evaluation) ${ }^{16}$ e VADT (veterans affairs dia- 
betes trial $)^{17}$ che se pure non hanno evidenziato alcun aumento della mortalità, non hanno tuttavia dimostrato alcun effetto benefico da parte di un controllo intensivo della glicemia sugli outcome macrovascolari compositi. Inoltre, il controllo glicemico intensivo ha determinato un aumento di peso ed episodi di ipoglicemia grave da 2 a 3 volte superiori.

Da sottolineare, però, che le popolazioni degli studi erano diverse: nell'UKPDS i pazienti erano diabetici di nuova diagnosi, negli altri studi erano diabetici di vecchia data, e molti con patologia cardiovascolare già in atto e comunque fragili. Ciò confermerebbe che $\mathrm{i}$ benefici del controllo glicemico sono massimi solo se la terapia ipoglicemizzante aggressiva viene attuata all'inizio della malattia, mentre si perdono o si attenuano quando la malattia ha già avuto il tempo di produrre danni più o meno manifesti. ${ }^{18,19}$

La Cochrane Collaboration ${ }^{20}$ ha rivisitato le evidenze riguardo alle due strategie e gli autori della revisione, pur evidenziando che i bias degli studi sono numerosi, sono giunti alla conclusione che uno stretto controllo glicemico riduce le complicanze microvascolari, ma non quelle macrovascolari.

Le ultime linee guida dell'American Diabetes Association e dell'European Association for the Study of Diabetes, non prendono una posizione definita, e pur consigliando un target di emoglobina glicosilata di $6,5 \%$, riconoscono che la terapia ipoglicemizzante va modulata in base al tipo di paziente, considerando le comorbilità, l'aspettativa di vita e il rischio di ipoglicemie gravi. ${ }^{21}$

Le linee guida, recependo le indicazioni provenienti dalle evidenze scientifiche, consigliano un approccio personalizzato: un controllo glicemico più stringente e valori di emoglobina glicosilata inferiori a $7 \%$, preferibilmente attorno al $6,5 \%$ sono indicati per pazienti diabetici di nuova diagnosi e/o giovani e/o con lunga aspettativa di vita. Valori di emoglobina glicosilata meno stringenti $(7,5-8 \%)$ e un controllo glicemico meno rigido sono indicati per pazienti diabetici con lunga storia di diabete e/o anziani e/o con una aspettativa di vita ridotta e/o affetti da precedenti patologie cardiovascolari e/o fragili e/o affetti da diverse comorbilità e/o con frequenti e severe ipoglicemie. Inoltre, secondo i risultati dello studio di Yau e coll., 22 potrebbe essere ragionevole nei soggetti particolarmente anziani mantenere un valore di glicosilata compreso tra 8 e $9 \%$.

Certamente ogni organizzazione professionale ribadisce l'importanza dell'individuazione di obiettivi glicemici, ma fornisce scarse indicazioni su come questa dovrebbe essere fatta. Stabilire un obiettivo individuale richiede la considerazione di 2 set di variabili, le caratteristiche cliniche e quelle psico-socio-economiche, entrambe specifiche per ogni paziente, laddove l'impostazione psico-socio-economica svolge spesso un ruolo determinante nella definizione degli obiettivi.
A tal proposito, numerose linee-guida per la prevenzione cardiovascolare nel paziente diabetico sottolineano l'importanza della valutazione del rischio cardiovascolare globale (età, genere, familiarità per coronaropatia o morte improvvisa, attività fisica, fumo, peso corporeo e distribuzione del grasso corporeo, durata della malattia diabetica, controllo glicemico, pressione arteriosa, microalbuminuria, lipidi plasmatici), nonché della stratificazione del rischio mediante l'utilizzo di specifici algoritmi. ${ }^{23-28}$ Gli algoritmi attualmente disponibili per il calcolo del rischio cardiovascolare presentano, però, dei limiti sostanziali in quanto non tengono conto della durata della malattia diabetica, né del grado di compenso metabolico. Inoltre non sono ottimali perché sono stati disegnati per popolazioni a più elevato rischio cardiovascolare rispetto alla popolazione italiana. ${ }^{4,726,29,30}$

Numerosi studi hanno correlato il rischio cardiovascolare al compenso glicemico. In essi, sebbene numerosi elementi di criticità (possibile publication bias, esiguo numero di studi disponibili, eterogeneità degli studi stessi) suggeriscano la necessità di ulteriori conferme di tali dati, ${ }^{31}$ è interessante l'osservazione di un'associazione tra compenso glicemico e macroangiopatia, meno forte di quella riscontrata con la microangiopatia, che conferma i risultati dell'UKPDS. . $^{32-36}$

Nel 2001 i ricercatori del UKPDS hanno formulato un algoritmo nel quale vengono presi in considerazione sia la durata della malattia sia il valore di HbAlc. ${ }^{37}$ Le linee-guida dell'International Diabetes Federation (IDF), ${ }^{38}$ sulla base delle considerazioni sopra esposte, considerano questo algoritmo come il più idoneo per la popolazione diabetica.

Un modello di screening è stato sviluppato nell'ambito del Framingham Heart Study a partire dal concetto di fattore di rischio di coronary heart disease (CHD) inteso come ogni singolo fattore che abbia evidenziato una relazione causale diretta e indipendente con la coronaropatia, che è comunemente presente nelle popolazioni e che, quando modificato, ha reso possibile la riduzione del rischio di CHD. Nel Framingham i fattori causali modificabili (fumo, ipertensione, ipercolesterolemia e diabete) giustificano circa il 50\% della variabilità del rischio nell'ambito dei gruppi ad alto rischio per CHD; inoltre, questi quattro fattori possono giustificare, nel confronto con i soggetti a basso rischio, più del $90 \%$ dell'eccesso di rischio per CHD. ${ }^{39}$

Nell'ambito dello studio DAI è stata valutata l'entità del rischio di cardiopatia ischemica in un campione di pazienti diabetici di tipo 2 inclusi nello studio. ${ }^{39}$ Erano pazienti tra 40 e i 70 anni e si è visto che la popolazione più giovane presentava percentualmente un rischio molto minore che però nella proiezione a 60 anni diventava molto più alto: in conclusione la popolazione studiata era soggetta ad un elevato rischio di cardiopatia ischemica sia nel breve che nel lungo termine. 
Pertanto, sulla base di quanto sopra, si può convenire che è giusto effettuare una stratificazione del rischio cardiovascolare anche nei pazienti diabetici senza evidenza di complicanze cardiovascolari in base all'età (>40 anni) e alla presenza di uno o più fattori di rischio. In alternativa, si possono utilizzare i vari algoritmi a disposizione (score, Carte del rischio italiane) preferendo l'UKPDS Engine, che ha il vantaggio di considerare sia la durata del diabete che il grado di compenso e che recentemente è stato validato anche in una popolazione di diabetici italiani. ${ }^{40}$

Certamente il diabete tipo 2 è un fattore di rischio indipendente per le patologie macrovascolari, ma condizioni coesistenti quali, per es., l'ipertensione, la dislipidemia, il fumo di sigaretta, sono altrettanti fattori di rischio cardiovascolari indipendenti. Perciò, considerando la molteplicità dei fattori di rischio cardiovascolare presenti nel diabete e le loro interazioni, è importante sottolineare che i risultati più importanti, in termini di riduzione della mortalità totale, della mortalità cardiovascolare e degli eventi cardiovascolari (oltre il 50\%) si sono ottenuti con un intervento intensivo teso all'ottimizzazione di tutti i fattori di rischio cardiovascolare. ${ }^{41-43}$ Gaede $^{42}$ e collaboratori hanno evidenziato, in un periodo di follow-up medio di 7,8 anni, che a fronte di cure standard, una combinazione intensiva di interventi comportamentali e farmacologici nel diabete di tipo 2 riduceva l'incidenza di malattie cardiovascolari del 53\%, del 61\% di nefropatia, del $58 \%$ di retinopatia e del $73 \%$ di neuropatia autonomica.

\section{Ictus}

Nelle recenti linee guida congiunte dell'American Heart Association (AHS)/American Stroke Association (ASA) ${ }^{44}$ per la prevenzione primaria dell'ictus, una sezione è specificamente dedicata alle persone con diabete che viene definito un fattore di rischio indipendente per patologia cardiovascolare e in particolare per l'ictus. Il paziente diabetico, in particolare, presenta un rischio 2-3 volte superiore, rispetto al non diabetico, di andare incontro a evento cerebrovascolare. Secondo una recente metanalisi, però, stratificando i pazienti diabetici sottoposti a trattamento glicemico intensivo si otteneva una riduzione significativa dell'incidenza di stroke nel sottogruppo degli obesi con indice di massa corporea $>30$. Da qui la necessità di considerare l'ictus, accidente cerebrovascolare acuto, esito della progressione di molteplici fattori sui quali dobbiamo intervenire più precocemente possibile con un approccio più tempestivo ed aggressivo. Nello studio Steno- $2^{45}$ è stato confrontato l'effetto di un mirato ed intensificato intervento multifattoriale con un trattamento convenzionale sui fattori di rischio cardiovascolare modificabili nei pazienti con diabete di tipo 2 e microalbuminuria. Il calo dei valori di emo- globina glicosilata, della pressione sistolica e diastolica, dei livelli di colesterolo e trigliceridi e il tasso di escrezione di albumina erano tutti significativamente maggiore nel gruppo ad alta intensità di terapia che nel gruppo terapia convenzionale.

Pertanto, solo un approccio globale alla malattia, non limitato al solo controllo glicemico, ma esteso alle diverse componenti del rischio cardiovascolare, può consentirci di ridurre in maniera clinicamente significativa l'impatto delle complicanze macroangiopatiche sulla mortalità e morbilità del diabete.

\section{Prevenzione non farmacologica: ruolo dell'alimentazione e dell'attività fisica}

\section{Terapia nutrizionale (dieta)}

È opportuno passare dal concetto di dieta, con prescrizione al paziente diabetico di uno schema alimentare più o meno standard, a quello di terapia nutrizionale: individualizzata per ogni paziente, che tenga conto dell'età e delle comorbilità, prescritta da operatori specificamente competenti, accompagnata da un intervento educazionale sul paziente e/o su un eventuale caregiver.

Ciò costituisce una componente fondamentale della terapia e della gestione del paziente diabetico, sia in prevenzione primaria che secondaria, con un rilevante impatto sul controllo glicemico (riduzione di HbAlc di $0,3-1 \%$ nel diabete tipo $1,{ }^{46-48}$ e di $0,5-2 \%$ nel tipo 2). ${ }^{49-52}$

Nei pazienti diabetici sovrappeso-obesi è raccomandata la riduzione dell'intake calorico; i dati di letteratura indicano però che un significativo calo ponderale si ottiene solo affiancando all'approccio dietetico un intervento intensivo sullo stile di vita che preveda un follow up molto stretto. ${ }^{53,54}$

Vari approcci dietetici si sono dimostrati efficaci nel diabete, ${ }^{54-59} \mathrm{ma}$ in realtà è necessario che le diete vengano modellate sul singolo paziente tenendo conto non solo degli obiettivi metabolici (target di HbAlc e frazioni lipidiche), ma anche, se possibile, di preferenze, abitudini alimentari e possibilità di aderire alle indicazioni. ${ }^{46,60}$

\section{Intake proteico}

Nei pazienti con nefropatia diabetica e albuminuria la riduzione dell'intake proteico al di sotto del normale non è raccomandata, in quanto ciò non ha effetti sul peggioramento della filtrazione glomerulare, sul controllo glicemico o sul rischio cardiovascolare. ${ }^{61,62}$

\section{Intake lipidico}

Intake lipidico può essere riassunto come segue: i) l'intake ideale di lipidi nel paziente diabetico non è chiaramente definibile sulla base di evidenze; può essere preso come riferimento un valore del $20-35 \%$ delle 
calorie totali, ${ }^{63}$ ii) il tipo di acidi grassi assunti (monoinsaturi meglio dei polinsaturi) ${ }^{54,64,65}$ pare influire più della quantità totale sui parametri metabolici e sul rischio cardiovascolare, ${ }^{54,63,66}$ iii) la supplementazione con acidi grassi omega-3 ad alto dosaggio riduce la trigliceridemia nei diabetici di tipo $2,{ }^{67}$ ma non è efficace in prevenzione $\mathrm{CV}$ primaria o secondaria. ${ }^{68-70}$

\section{Intake sodio}

L'apporto di sodio raccomandato nel paziente diabetico dev'essere, secondo gli standard di cura 2015 dell'American Diabetes Association, ${ }^{71}$ inferiore a 2,3 $\mathrm{g} / \mathrm{die}$, analogamente a quanto consigliato nella popolazione generale (grado di evidenza: B); nel paziente ad alto rischio va considerata l'opportunità di una ulteriore riduzione individualizzata.

\section{Attività fisica}

L'attività fisica viene fortemente raccomandata nel paziente diabetico. L'esercizio fisico regolare migliora il controllo glicemico e i fattori di rischio cardiovascolare, facilita la perdita di peso, migliora il benessere soggettivo. ${ }^{72-74}$

Un diabetico adulto dovrebbe svolgere almeno 150 $\mathrm{min} / \mathrm{settimana} \mathrm{di} \mathrm{attività} \mathrm{fisica} \mathrm{aerobica} \mathrm{di} \mathrm{intensità} \mathrm{mo-}$ derata (al 50-70\% della frequenza massima teorica), suddivisi in almeno tre giorni diversi. È inoltre importante tentare di ridurre la sedentarietà, in particolare evitando di rimanere seduti a lungo (più di $90 \mathrm{~min}$ ) in attività come lavorare al computer o guardare la TV..$^{75}$

In prevenzione secondaria l'esercizio fisico strutturato come intervento terapeutico, anche nel paziente diabetico, costituisce da tempo una delle componenti fondamentali. ${ }^{76,77}$ Per la maggior parte dei pazienti è raccomandato l'esercizio fisico di tipo aerobico, di intensità bassa o moderata, adattato all'età ed al diverso livello di capacità di esercizio di ciascun paziente. Naturalmente pazienti con cardiopatia clinicamente instabile dovrebbero essere esclusi da un programma di esercizio fisico.

Nella maggior parte dei casi la stratificazione del rischio clinico basata sulla storia clinica, l'esame fisico, l'elettrocardiogramma (ECG) a riposo ed un test di capacità funzionale (es., il 6 minute walking test) sono sufficienti per avviare in sicurezza un programma di attività fisica. ${ }^{78,79}$

Sono però da considerare ad alto rischio i pazienti: i) con IM complicato da scompenso cardiaco, shock cardiogeno e/o aritmie ventricolari complesse; ii) con angina o dispnea a basso livello di attività fisica (es., entro i primi quattro minuti del 6 minute walking test; iii) con una depressione del segmento ST maggiore o uguale ad $1 \mathrm{~mm}$ nell'ECG a riposo.

Nei diabetici inoltre esistono condizioni particolari che impongono grande cautela: i) un'attività fi- sica intensa è controindicata nei diabetici di tipo 1 chetosici, in quanto può peggiorare la chetosi; ${ }^{80}$ ii) nei diabetici in terapia con insulina o secretagoghi l'attività fisica può determinare ipoglicemia se la posologia dei farmaci non viene ridotta o la dose di carboidrati non viene aumentata in vista dell' esercizio fisico (utile consumare carboidrati prima dell'esercizio se la glicemia è $<100 \mathrm{mg} / \mathrm{dL}$ ); iii) in pazienti con retinopatia proliferativa o non proliferativa severa l'attività fisica vigorosa è controindicata per il rischio di indurre distacco di retina o emorragia intravitreale, ${ }^{81}$ iv) nei pazienti con neuropatia periferica non grave $150 \mathrm{~min} / \mathrm{settimana}$ di attività fisica moderata migliorano gli outcomes: ${ }^{81}$ è però necessario l'utilizzo di calzature adeguate e un esame giornaliero del piede per evidenziare eventuali lesioni; v) la neuropatia autonomica è un fattore di rischio per morte cardiovascolare ed ischemia silente, ed i pazienti che ne sono affetti presentano ridotta risposta cardiovascolare all'esercizio, ipotensione posturale, ridotta capacità di termoregolazione e di visione notturna, maggiore suscettibilità all'ipoglicemia. È perciò necessaria una attenta valutazione cardiologica prima di avviare eventualmente il paziente all'attività fisica; ${ }^{82,83} \mathrm{vi}$ ) l'attività fisica può in acuto aumentare la proteinuria, ma non vi è alcuna dimostrazione che ciò aggravi la progressione della nefropatia diabetica, per cui in questi soggetti non sono necessarie restrizioni particolari. ${ }^{81}$

\section{Prevenzione farmacologica}

\section{Terapia dell'iperglicemia}

Alla luce dei tre grandi studi di intervento quali ACCORD,${ }^{15}$ ADVANCE ${ }^{16}$ e VADT,,${ }^{17}$ appare non opportuno e addirittura molto rischioso ricercare uno stretto controllo glicemico con un trattamento farmacologico intensivo in pazienti diabetici ad elevato rischio cardiovascolare, probabilmente a causa di un aumentato rischio di ipoglicemie gravi, capaci di indurre eventi di tipo ischemico e/o aritmico.

Pertanto, nel paziente con diabete tipo 2, in prevenzione secondaria, spesso un paziente complesso, polipatologico, anziano e fragile, il target va individualizzato attraverso un ragionamento clinico articolato, che tenga conto nel singolo paziente di molteplici fattori.

Due sono i punti fondamentali da considerare: i) la corretta stima dell'aspettativa di vita del paziente, in considerazione dell'età, della gravità della patologia cardiovascolare, del numero e gravità delle comorbilità; ii) il rischio di ipoglicemia, in considerazione della terapia ipoglicemizzante in atto (ottimizzata cercando di privilegiare l'uso di farmaci che non comportano rischio di ipoglicemia) e della aderenza 
terapeutica, della capacità di autogestione, della presenza ed adeguatezza di un caregiver.

Una proposta di identificazione del target di $\mathrm{HbA1c}$ nel paziente diabetico in prevenzione secondaria è indicata nella Tabella 1.

Per quanto riguarda i vari farmaci ipoglicemizzanti, in prevenzione cardiovascolare secondaria è ovviamente da preferire, per le ragioni già sottolineate, l'utilizzo di farmaci a basso rischio di ipoglicemia. Per quanto concerne la safety cardiovascolare dei farmaci ipoglicemizzanti, nessuno di essi ha dimostrato di poter di per sé ridurre il rischio cardiovascolare nel diabetico, se non la metformina, che nello studio UKPDS (peraltro ormai datato, e su una piccola casistica di pazienti naive) ha ridotto la mortalità coronarica rispetto alla terapia convenzionale. ${ }^{11}$

Essa rimane perciò il farmaco di prima scelta in prevenzione secondaria se non sono presenti controindicazioni al suo impiego (insufficienza renale, scompenso cardiaco e/o condizioni che predispongano all'acidosi lattica).

Il pioglitazone ha invece mostrato in varie osservazioni di aumentare il rischio di scompenso cardiaco, ${ }^{84}$ anche se non la mortalità per scompenso cardiaco.

Anche un inibitore del DPP-IV, il saxagliptin, ha mostrato nello studio SAVOR-TIMI 53 di aumentare il rischio di ospedalizzazione per scompenso cardiaco (ma non la mortalità) ${ }^{85}$

Dati neutri sul rischio CV sono disponibili con l'insulina glargine. ${ }^{86}$

È proprio di questi giorni la pubblicazione dello studio TECOS ${ }^{87}$ che valuta l'aggiunta di sitagliptin alle terapie tradizionali e che, dai risultati ottenuti, non sembra aumentare il rischio di eventi avversi cardiovascolari maggiori, ospedalizzazione per insufficienza cardiaca, o altri eventi avversi.

Sono attualmente in corso numerosi trial che dovrebbero chiarire in modo definitivo gli aspetti relativi alla safety cardiovascolare dei più recenti ipoglicemizzanti (inibitori del DPP-IV, agonisti del GLP-1, SGLT2 inibitori).

\section{Terapia dell'ipertensione arteriosa}

I trial di intervento hanno chiaramente dimostrato il beneficio di ridurre la pressione arteriosa nel diabetico a valori inferiori a $140 \mathrm{mmHg}$ per la pressione sistolica (PAS) e a $90 \mathrm{mmHg}$ per la pressione diastolica (PAD), in termini di riduzione di eventi coronarici, stroke, nefropatia diabetica ${ }^{88} \mathrm{Vi}$ sono invece limitate evidenze di beneficio per target pressori inferiori.

Sulla base dei dati dello studio ACCORD e delle metanalisi dei principali megatrial di intervento, ${ }^{89-91}$ le linee guida internazionali hanno recentemente rivisto al rialzo i target pressori nel paziente diabetico, fino al 2013 individuati in $<130 / 80 \mathrm{mmHg}$. ${ }^{92-94}$

Le linee guida ESH/ESC del 2013 raccomandano un target di $<140 \mathrm{mmHg}$ per la PAS nei pazienti diabetici, indipendentemente dal livello di rischio $\mathrm{CV}$ (Raccomandazione di grado IA).

L'obiettivo raccomandato per la PAD è $<85 \mathrm{mmHg}$ (verificando che valori di PAD tra 80 e $85 \mathrm{mmHg}$ siano ben tollerati e privi di effetti collaterali). ${ }^{95}$

Le recentissime indicazioni degli standard di cura 2015 dell'American Diabetes Association raccomandano per i pazienti diabetici ipertesi un target $<140$ mmHg per la PAS e $<90 \mathrm{mmHg}$ per la PAD (Grado di evidenza: A). Target inferiori ( $<130 \mathrm{mmHg}$ PAS, $<80$ $\mathrm{mmHg}$ PAD) possono essere perseguiti in alcuni soggetti (ad es. soggetti giovani, con lunga aspettativa di vita, o soggetti ad elevato rischio di stroke-PAS o con malattia renale cronica ed albuminuria-PAD), soprattutto se possono essere raggiunti con l'impiego di pochi farmaci e senza effetti collaterali della terapia. ${ }^{71}$

Indicazioni sostanzialmente analoghe giungono dall'Eighth Joint National Committee (diabetici $>18$ anni: PAS $<140 \mathrm{mmHg}$, PAD $<90 \mathrm{mmHg}){ }^{96}$

Le linee guida suggeriscono che la terapia farmacologica dell'ipertensione nel paziente diabetico debba comprendere un ACE-inibitore o un recettore dell'angiotensina (Grado di evidenza: C), ${ }^{71,93,97,98} \mathrm{ma}$ in realtà l'identificazione del farmaco antipertensivo di prima scelta da impiegare nel paziente diabetico è,

Tabella 1. Target di emoglobina glicosilata nel paziente diabetico in prevenzione secondaria.

\begin{tabular}{llll}
\hline $\begin{array}{l}\text { Giudizio clinico sull'aspettativa } \\
\text { di vita* }\end{array}$ & $\begin{array}{l}\text { Terapia solo con farmaci a basso } \\
\text { rischio di ipoglicemia }\end{array}$ & $\begin{array}{l}\text { Terapia con farmaci ad alto } \\
\text { rischio di ipoglicemia }\end{array}$ & $\begin{array}{l}\text { Terapia con farmaci ad alto rischio } \\
\text { di ipoglicemia }^{\#}\end{array}$ \\
$\begin{array}{l}\text { Questo paziente probabilmente } \\
\text { vivrà... }\end{array}$ & $\begin{array}{l}\text { In pazienti con buona capacità } \\
\text { di autogestione (o presenza di } \\
\text { caregiver adeguato) }\end{array}$ & $\begin{array}{l}\text { In pazienti con scarsa capacità } \\
\text { di autogestione (e assenza di } \\
\text { caregiver adeguato) }\end{array}$ \\
\hline$<5$ anni & $<\%$ & $8-8.5 \%$ & $8-9 \%^{\S}$ \\
\hline $5-10$ anni & $<7 \%$ & $7-8 \%$ & $8-8.5 \% \%^{\S}$ \\
\hline 10 anni & $<7 \%$ & $<7 \%$ & $7-8 \%$ \\
\hline
\end{tabular}

*In base ad età, gravità della patologia cardiovascolare, numero e gravità delle comorbilità; ${ }^{\circ}$ metformina, acarbose, DPP-IV antagonisti, GLP-1 agonisti, SGLT-2 inibitori; ${ }^{*}$ sulfaniluree, glinidi, insulina; ' una A1C di 8,5\% equivale ad una glicemia media stimata di circa $200 \mathrm{mg} / \mathrm{dL}$; target più elevati espongono al rischio di glicosuria, disidratazione, sindrome iperglicemica iperosmolare. 
dal punto di vista clinico, poco rilevante: $i$ target pressori infatti sono quasi sempre difficilmente raggiungibili e solo con una combinazione di più farmaci: nei principali trials clinici su diabetici la media dei farmaci utilizzati è $>3 .{ }^{99}$

\section{Terapia dell'ipercolesterolemia e delle dislipidemie}

Numerosi trial clinici hanno definitivamente dimostrato l'efficacia della terapia con statine sugli outcomes $\mathrm{CV}$ in prevenzione secondaria, ed in particolare nei pazienti diabetici..$^{71,100-109}$

La Tabella 2 presenta una proposta di trattamento con statine per il paziente diabetico in prevenzione secondaria o con significativi fattori di rischio $\mathrm{CV}$ associati (derivata dall'American Diabetes Association ${ }^{71}$ ed adattata alla realtà italiana).

Se con le dosi massime tollerate di statine non si raggiunge una significativa riduzione del colesterolo con lipoproteine a bassa densità ( $-30 \%$ dal baseline) l'aggiunta di niacina, fenofibrato, ezetimide o sequestranti degli acidi biliari può determinare una ulteriore riduzione; non vi è però chiara evidenza che ciò comporti un beneficio in termini di riduzione di eventi CV. ${ }^{110}$

Bassi valori di colesterolo con lipoproteine ad alta densità ed elevati livelli di trigliceridi costituiscono le alterazioni lipidiche più tipiche nel diabete tipo 2 . Non vi è chiara evidenza che il loro specifico trattamento farmacologico sia utile, ${ }^{111,112}$ in particolare nei pazienti ad alto rischio $\mathrm{CV}$ o in prevenzione secondaria. ${ }^{113}$

\section{Terapia con antiaggreganti piastrinici}

È controverso se l'aspirina in prevenzione primaria abbia un effetto protettivo sugli eventi CV, sia nei dia- betici che nei non diabetici. È viceversa assodato l'effetto di riduzione degli eventi e della mortalità $\mathrm{CV}$ della terapia con aspirina in prevenzione secondaria (pregresso IMA o stroke), sia nei diabetici che nei non diabetici. ${ }^{14-117}$

Le recentissime raccomandazioni dell'American Diabetes Association ${ }^{71}$ prevedono che in prevenzione primaria la terapia con aspirina (75-162 $\mathrm{mg} / \mathrm{die})$ venga presa in considerazione nel diabete tipo 1 o tipo 2 per pazienti con un rischio $\mathrm{CV}$ elevato ( $>10 \%$ a 10 anni), cioè nella massima parte di maschi $>50$ anni e femmine $>60$ anni con almeno un altro fattore di rischio maggiore cioè familiarità per CVD, ipertensione, fumo, dislipidemia, albuminuri (Forza della raccomandazione: C). In pazienti diabetici a minor rischio CV la terapia con aspirina non è raccomandata in quanto il rischio di sanguinamento verosimilmente supera i potenziali benefici (Forza della raccomandazione: $\mathrm{C}$ ).

In prevenzione secondaria (pazienti diabetici con pregresso evento $\mathrm{CV}$ ) la terapia con aspirina (75-162 $\mathrm{mg} / \mathrm{die}$ ) è invece fortemente raccomandata (Forza della raccomandazione: A); in caso di documentata allergia all'aspirina va utilizzato il clopidogrel (75 $\mathrm{mg} /$ die) (Forza della raccomandazione: B). La doppia antiaggregazione è considerata ragionevole fino ad un anno dopo una sindrome coronarica acuta (Forza della raccomandazione: $\mathrm{C}$ ).

Le dosi di aspirina utilizzate nei trial su pazienti diabetici variano fra 100 e $325 \mathrm{mg} / \mathrm{die}$; non vi sono evidenze che inducano a preferire un particolare dosaggio, e verosimilmente l'utilizzo di una dose più bassa riduce il rischio di effetti collaterali. ${ }^{118}$

È noto che la funzione piastrinica è alterata nei pazienti diabetici, nei quali il fenomeno della resistenza all'aspirina è di frequente riscontro; queste osserva-

Tabella 2. Raccomandazioni per il trattamento con statine nei pazienti diabetici a rischio cardiovascolare.

\begin{tabular}{|c|c|c|c|}
\hline Età & Rischio CV & Dose di statina raccomandata & Monitoraggio di c-LDL \\
\hline$<40$ anni & $\begin{array}{l}\text { Presenti significativi fattori } \\
\text { di rischio } \mathrm{CV}^{*}\end{array}$ & Moderata & $\begin{array}{l}\text { Quando necessario a monitorare } \\
\text { l'aderenza al trattamento }\end{array}$ \\
\hline Prevenzione secondaria $^{\circ}$ & & Elevata & $\begin{array}{l}\text { Quando necessario a monitorare } \\
\text { l'aderenza al trattamento }\end{array}$ \\
\hline 40-75 anni & $\begin{array}{l}\text { Presenti significativi fattori } \\
\text { di rischio } \mathrm{CV}^{*}\end{array}$ & Elevata & $\begin{array}{l}\text { Quando necessario a monitorare } \\
\text { l'aderenza al trattamento }\end{array}$ \\
\hline Prevenzione secondaria ${ }^{\circ}$ & & Elevata & $\begin{array}{l}\text { Quando necessario a monitorare } \\
\text { l'aderenza al trattamento }\end{array}$ \\
\hline$>75$ anni & $\begin{array}{l}\text { Presenti significativi fattori } \\
\text { di rischio } \mathrm{CV}^{*}\end{array}$ & Moderata & $\begin{array}{l}\text { Quando necessario a monitorare } \\
\text { l'aderenza al trattamento }\end{array}$ \\
\hline Prevenzione secondaria $^{\circ}$ & & Elevata & $\begin{array}{l}\text { Quando necessario a monitorare } \\
\text { l'aderenza al trattamento }\end{array}$ \\
\hline
\end{tabular}

$\mathrm{CV}$, cardiovascolare; c-LDL, colesterolo con lipoproteine a bassa densità. *Uno o più fra: c-LDL $>100 \mathrm{mg} / \mathrm{dL}$, ipertensione, fumo, obesità; ${ }^{\circ}$ pregresso evento cardiovascolare maggiore o sindrome coronarica acuta. Modificata da American Diabetes Association, 2015. ${ }^{71}$ 
zioni non sono però di per sé sufficienti, in assenza di evidenze cliniche, a supportare la raccomandazione di utilizzare un dosaggio più elevato di aspirina.

Dopo una sindrome coronarica acuta in un paziente diabetico va utilizzata per almeno un anno la doppia antiaggregazione con aspirina + un agonista del recettore P2Y12 (ticagrelor o clopidogrel se non è stata eseguita un'angioplastica, altrimenti clopidogrel, ticagrelor o prasugrel). ${ }^{119}$

\section{Take home messages}

\section{Quali modalità di valutazione del rischio e di stratificazione prognostica}

I pazienti diabetici con evidenza clinica o strumentale di complicanze cardiovascolari sono da considerare a rischio cardiovascolare elevato/molto elevato.

I pazienti diabetici senza evidenza clinica o strumentale di complicanze cardiovascolari sono da considerare a rischio cardiovascolare elevato in base all'età (>40 anni), alla durata del diabete e alla presenza di uno o più fattori di rischio cardiovascolare.

Le linee guida consigliano un approccio personalizzato al paziente diabetico con alto rischio $\mathrm{CV}$ che tenga conto dell'età del paziente, della gravità della patologia cardiovascolare, del numero e gravità delle comorbilità, del rischio di ipoglicemia.

Quali azioni da implementare, ritagliate sul singolo paziente?

\section{Target glicemici}

Controllo glicemico stretto e valori di emoglobina glicosilata preferibilmente attorno al $6,5 \%$ sono indicati per pazienti diabetici di nuova diagnosi e/o giovani e/o con lunga aspettativa di vita.

Valori di emoglobina glicosilata meno stringenti (7,5-8\%) e un controllo glicemico meno stretto sono indicati per pazienti diabetici con lunga storia di diabete e/o anziani e/o affetti da precedenti patologie cardiovascolari e/o fragili e/o affetti da importanti comorbidità e/o con frequenti e severe ipoglicemie.

Valori di emoglobina glicosilata ancora meno stringenti (8-9\%) sono indicati per pazienti diabetici ultraottantenni e con perdita dell'autonomia con grave rischio di ipoglicemia.

\section{Modificazioni dello stile di vita}

È preferibile mirare non ad una dieta ma da una terapia nutrizionale individualizzata.

L'attività fisica è fortemente raccomandata nel paziente diabetico. L'esercizio fisico regolare migliora il controllo glicemico e i fattori di rischio cardiovascolare, facilita la perdita di peso, migliora il benessere soggettivo.

\section{Terapia di alcune condizioni cliniche associate (per altre condizioni cliniche associate si rimanda ai rispettivi capitoli)}

\section{Ipertensione arteriosa}

L'iperteso diabetico necessita normalmente di un trattamento antiipertensivo aggressivo, con un target $<140 / 85-90 \mathrm{mmHg}$, valutando sempre la tollerabilità individuale. Nella maggior parte dei casi è necessaria una terapia di associazione.

Non esiste una classe di farmaci antipertensivi con una dimostrata superiorità rispetto ad un'altra classe in termini di riduzione degli eventi cardiovascolari, ma gli inibitori del sistema renina angiotensina andrebbero sempre impiegati, quando possibile.

\section{Dislipidemie}

IIn prevenzione secondaria le statine andrebbero sempre impiegate e a dosaggi adeguati.

\section{Aspirina e terapia antiaggregante}

In prevenzione primaria la terapia con aspirina $(75-162 \mathrm{mg} / \mathrm{die})$ è da prendere in considerazione nel diabete tipo 1 o tipo 2 per pazienti con un rischio $\mathrm{CV}$ elevato ( $>10 \%$ a 10 anni) (Forza della raccomandazione: $\mathrm{C}$ ). In pazienti diabetici a minor rischio $\mathrm{CV}$ la terapia con aspirina non è raccomandata in quanto il rischio di sanguinamento verosimilmente supera i potenziali benefici (Forza della raccomandazione: $\mathrm{C}$ ).

In prevenzione secondaria è fortemente raccomandata (Forza della raccomandazione: A).

\section{Bibliografia}

1. Haffner SM, Lehto S, Ronnemaa T, et al. Mortality from coronary heart disease in subjects with type 2 diabetes and in nondiabetic subjects with and without prior myocardial infarction. N Engl J Med 1998;339:229-34.

2. Clarke R, Emberson J, Fletcher A, et al. Life expectancy in relation to cardiovascular risk factors: 38 year followup of 19,000 men in the Whitehall study. BMJ 2009;339: b3513.

3. Bruno G, Picariello R, Petrelli A, et al. Direct costs in diabetic and non diabetic people: the population-based Turin study, Italy. Nutr Metab Cardiovasc Dis 2012; 22:684-90.

4. Istituto Superiore di Sanità. Il Progetto Cuore; 2015. Disponibile su: http://www.cuore.iss.it

5. Bruno G, Merletti F, Boffetta P, et al. Impact of glycaemic control, hypertension and insulin treatment on general and cause-specific mortality: an Italian population-based cohort of type II (non-insulin-dependent diabetes mellitus. Diabetologia 1999;42:297-301.

6. Brun E, Nelson RG, Bennett PH, et al. Verona Diabetes Study. Diabetes duration and cause-specific mortality in the Verona Diabetes Study. Diabetes Care 2000;23:1119-23.

7. Associazione Medici Diabetologi (AMD), Società Ita- 
liana di Diabetologia (SID). Standard italiani per la cura del diabete mellito 2014; 28 maggio 2014. Disponibile su: http://www.standarditaliani.it

8. Vaccaro O, Eberly LE, Neaton JD, et al. Impact of diabetes and previous myocardial infarction on long-term survival: 25 -year mortality follow-up of primary screenees of the Multiple Risk Factor Intervention Trial. Arch Intern Med 2004;164:1438-43.

9. Sattar N. Revisiting the links between glycaemia, diabetes and cardiovascular disease. Diabetologia 2013;56: 686-95.

10. U.K. Prospective Diabetes Study 1998. Intensive blood glucose control with sulfonylureas or insulin compared with conventional treatment and risk for complications in patients with type 2 diabetes (UKPDS 33). Lancet 1998;352:837-53.

11. [No authors listed]. The effect of intensive treatment of diabetes on the development and progression of longterm complications in insulin-dependent diabetes mellitus. The Diabetes Control and Complications Trial Research Group. N Engl J Med 1993;329:977-86.

12. Holman RR, Paul SK, Bethel MA, et al. 10-year followup of intensive glucose control in type 2 diabetes. $\mathrm{N}$ Engl J Med 2008;359:1577-89.

13. Aschner PJ, Ruiz AJ. Metabolic memory for vascular disease in diabetes. Diabetes Technol Ther 2012;14:S68-74.

14. Ceriello A, Ihnat MA, Thorpe JE. Clinical review 2: the "metabolic memory": is more than just tight glucose control necessary to prevent diabetic complications? J Clin Endocrinol Metab 2009;94:410-5.

15. The Action to Control Cardiovascular Risk in Diabetes Study Group. Effects of intensive glucose lowering in type 2 diabetes. N Engl J Med 2008;358:2545-59.

16. The ADVANCE Collaborative Group. Intensive blood glucose control and vascular outcomes in patients with type 2 diabetes. N Engl J Med 2008;358:2560-72.

17. Hayward RA, Reaven PD, Wiitala WL, et al. Follow-up of glycemic control and cardiovascular outcomes in type 2 diabetes. N Engl J Med 2015;372:2197-206.

18. Del Prato S. Mega-trials in type 2 diabetes. From excitement to frustration? Diabetologia 2009;52:1219-26.

19. Inzucchi SE, Bergenstal RM, Buse JB, et al. Management of hyperglycemia in type 2 diabetes: a patient-centered approach. Position statement of the American Diabetes Association (ADA) and the European Association for the Study of Diabetes (EASD). Diabetes Care 2012;35:1364.

20. Hemmingsen B, Lund SS, Gluud C, et al. Targeting intensive glycaemic control versus targeting conventional glycaemic control for type 2 diabetes mellitus. Cochrane Database Syst Rev 2013;(11):CD0081433.

21. Skyler JS, Bergenstal R, Bonow RO, et al. Intensive glycemic control and the prevention of cardiovascular events: Implications of the ACCORD, ADVANCE, and VA Diabetes trials: a position statement of the American Diabetes Association and a scientific statement of the American College of Cardiology Foundation and the American Heart Association. Diabetes Care 2009;32:187-92.

22. Yau CK, Eng C, Cenzer IS, et al. Glycosylated hemoglobin and functional decline in community-dwelling nursing home-eligible elderly adults with diabetes mellitus. J Am Geriatr Soc 2012;60:1215-21.

23. Linee-guida per la prevenzione cardiovascolare nel pa- ziente diabetico. A cura di AMD, SID, FAND, SIIA, FIC, SIMG, Forum per la prevenzione delle Malattie Cardiovascolari, SISA, Gruppo Cochrane Collaboration Italia. Il Diabete 2002;14:359-317. Disponibile su: http://sid.logicsolution.it/pubblicazioni/linee-guida/427--25032003prevenzione-delle-malattie-cardiovascolari-nel-diabete-ti po-1-e-tipo-2/download.html Accesso: 9/3/2015.

24. Canadian Diabetes Association Clinical Practice Guidelines Expert Committee. Canadian Diabetes Association 2008 - Clinical practice guidelines for prevention and management of diabetes in Canada. Canadian J Diabet 2008;32:S95-8.

25. National Collaboration Center for Chronic Conditions. Type 2 diabetes: national clinical guidelines for management in primary and secondary care (update). London: Royal College of Physicians; 2008.

26. Ministry of Health, New Zealand Guidelines Group. Management of type 2 diabetes best practice evidence based guideline; March 2003.

27. Pearson TA, Blair SN, Daniels SR, et al. AHA Guidelines for Primary Prevention of Cardiovascular Disease and Stroke: 2002 Update: Consensus Panel Guide to Comprehensive Risk Reduction for Adult Patients Without Coronary or Other Atherosclerotic Vascular Diseases. American Heart Association Science Advisory and Coordinating Committee. Circulation 2002;136:161-72.

28. Australian Centre for Diabetes Strategies Prince of Wales Hospital, Sydney for the Diabetes Australia Guideline Development Consortium. National evidence based guidelines for the management of type 2 diabetes mellitus; March 2004.

29. De Marco R, Locatelli F, Zoppini G, et al. Cause specific mortality in type 2 diabetes. The Verona Diabetes Study. Diabetes Care 1999;22:756-61.

30. Bruno G, Merletti F, Moffetta P, et al. Impact of glycemic control, hypertension and insulin-treatment on general and cause-specific mortalità. An Italia population-based cohort of type 2 diabetes. Diabetologia 1999;42:297-301.

31. Selvin E, Marinopoulos S, Berkenblit G, et al. Metaanalysis: glycosilated haemoglobin and cardiovascular disease in diabetes mellitus. Ann Inter Med 2004;141: 421-31.

32. Wei M, Gaskill SP, Haffner SM, Stern MP. Effects of diabetes and level of glycaemia on all-cause and cardiovascular mortality. The San Antonio Heart Study. Diabetes Care 1998;21:1167-72.

33. Uusitupa M, Niskanen LK, Siitonen O, et al. 5-year incidence of atherosclerotic vascular disease in relation to general risk factors, insulin level and abnormalities in lipoprotein composition in non-insulin-dependent diabetic and non diabetic subjects. Circulation 1990;82:27-36.

34. Mykkanen L, Laakso M, Uusitupa M, et al. Prevalence of diabetes and impaired glucose tolerance in elderly subjects and their association with obesity and family history of diabetes. Diabetes Care 1990;13:1099-105.

35. Lehto S, Ronnemaa T, Haffner SM, et al. Dyslipidemia and hyperglycaemia predict coronary heart disease events in middle-aged people with NIDDM. Diabetes 1997;46:1354-9.

36. Hu FB, Stampfer MJ, Solomon CG, et al. The impact of diabetes mellitus on mortality from all causes and coronary heart disease in woman. Arch Intern Med 2001; 161:1717-2173. 
37. The Oxford Centre for Diabetes, Endocrinology and Metabolism. UKPDS Risk Engine. Oxford: Isis Innovation Ltd; 2009. Available from: http://www.dtu.ox.ac.uk/ riskengine/download.php

38. International Diabetes Federation. Global guidelines for type 2 diabetes; 2005. Available from: http://www.idf. org/global-guideline-type-2-diabetes-2005

39. Grassi G, Monge L. Gli score di rischio cardiovascolare e la loro applicazione nello studio DAI. Ann Ist Super Sanità 2003;39:139-44.

40. Pagano E, Gray A, Rosato R, et al. Prediction of mortality and macrovascular complications in type 2 diabetes: validation of the UKPDS outcomes Model in the Casale Monferrato Survey, Italy. Diabetologia 2013;56:1726-34.

41. BARI 2D Study Group; Frye RL, August P, et al. A randomized trial of therapies for type 2 diabetes and coronary artery disease. N Engl J Med 2009;360:2503-15.

42. Gaede P, Vedel P, Larsen N, et al. Multifactorial intervention and cardiovascular disease in patients with type 2 diabetes. N Engl J Med 2003;348:383-93.

43. Gaede P, Lund-Andersen H, Parving HH, et al. Effect of multifactorial intervention on mortality in type 2 diabetes. N Engl J Med 2008;358:580-91.

44. Meschia JF, Bushnell C, Boden-Albala B, et al. Guidelines for the primary prevention of stroke: a statement for healthcare professionals from the American Heart Association/American Stroke Association. Stroke 2014;45:3754-832.

45. Nakagami T. Steno2 Study. Nihon Rinsho 2010;68:99106.

46. DAFNE Study Group. Training in flexible, intensive insulin management to enable dietary freedom in people with type 1 diabetes: dose adjustment for normal eating (DAFNE) randomized controlled trial. BMJ 2002; 325:746.

47. Laurenzi A, Bolla AM, Panigoni G, et al. Effects of carbohydrate counting on glucose control and quality of life over 24 weeks in adult patients with type 1 diabetes on continuous subcutaneous insulin infusion: a randomized, prospective clinical trial (GIOCAR). Diabetes Care 2011;34:823-7.

48. Scavone G, Manto A, Pitocco D, et al. Effect of carbohydrate counting and medical nutritional therapy on glycaemic control in type 1 diabetic subjects: a pilot study. Diabet Med 2010;27:477-9.

49. Wolf AM, Conaway MR, Crowther JQ, et al. Translating lifestyle intervention to practice in obese patients with type 2 diabetes: improving control with activity and nutrition (ICAN) study. Diabetes Care 2004;27: 1570-6.

50. Nield L, Moore H, Hooper L, et al. Dietary advice for treatment of type 2 diabetes mellitus in adults. Cochrane Database Syst Rev 2007;3:CD004097.

51. Coppell KJ, Kataoka M, Williams SM, et al. Nutritional intervention in patients with type 2 diabetes who are hyperglycaemic despite optimized drug treatment-Lifestyle Over and Above Drugs in Diabetes (LOADD) study: randomised controlled trial. BMJ 2010;341:c3337.

52. Franz MJ, Monk A, Barry B, et al. Effectiveness of medical nutrition therapy provided by dietitians in the management of non-insulindependent diabetes mellitus: a randomized, controlled clinical trial. J Am Diet Assoc 1995;95:1009-17.
53. Pi-Sunyer X, Blackburn G, Brancati FL, et al. Reduction in weight and cardiovascular disease risk factors in individuals with type 2 diabetes: one-year results of the Look AHEAD trial. Diabetes Care 2007;30: 1374-83.

54. Estruch R, Ros E, Salas-Salvadò J, et al. Primary prevention of cardiovascular disease with a Mediterranean diet. N Engl J Med 2013;368:1279-90.

55. Elhayany A, Lustman A, Abel R, et al. A low carbohydrate Mediterranean diet improves cardiovascular risk factors and diabetes control among overweight patients with type 2 diabetes mellitus: a 1-year prospective randomized intervention study. Diabetes Obes Metab 2010;12:204-9.

56. Azadbakht L, Fard NRP, Karimi M, et al. Effects of the dietary approaches to stop hypertension (DASH) eating plan on cardiovascular risks among type 2 diabetic patients: a randomized crossover clinical trial. Diabetes Care 2011;34:55-7.

57. Turner-McGrievy GM, Barnard ND, Cohen J, et al. Changes in nutrient intake and dietary quality among participants with type 2 diabetes following a lowfat vegan diet or a conventional diabetes diet for 22 weeks. J Am Diet Assoc 2008;108:1636-45.

58. Stern L, Iqbal N, Seshadri P, et al. The effects of lowcarbohydrate versus conventional weight loss diets in severely obese adults: one-year follow-up of a randomized trial. Ann Intern Med 2004;140:778-85.

59. Thomas D, Elliott EJ. Low glycaemic index, or low glycaemic load, diets for diabetes mellitus. Cochrane Database Syst Rev 2009;1:CD006296.

60. Delahanty LM, Nathan DM, Lachin JM, et al. Association of diet with glycated hemoglobin during intensive treatment of type 1 diabetes in the diabetes control and complications trial. Am J ClinNutr 2009;89:518-24.

61. Pan Y, Guo LL, Jin HM. Low-protein diet for diabetic nephropathy: a meta-analysis of randomized controlled trials. Am J ClinNutr 2008;88:660-6.

62. Robertson L, Waugh N, Robertson A. Protein restriction for diabetic renal disease. Cochrane Database Syst Rev 2007;4:CD002181.

63. Institute of Medicine of the National Academies. Dietary reference intakes for energy, carbohydrate, fiber, fat, fatty acids, cholesterol, protein, and amino acids; 2002. Available from: https://www.nal.usda.gov/fnic/DRI/DRI Energy/energy full report.pdf

64. Brehm BJ, Lattin BL, Summer SS, et al. Oneyear comparison of a high-monounsaturated fat diet with a highcarbohydrate diet in type 2 diabetes. Diabetes Care 2009;32:215-20.

65. Shai I, Schwarzfuchs D, Henkin Y, et al. Weight loss with a lowcarbohydrate, Mediterranean, or low-fat diet. N Engl J Med 2008;359:229-41.

66. Ros E. Dietary cis-monounsaturated fatty acids and metabolic control in type 2 diabetes. Am J ClinNutr 2003;78:617S-25S.

67. Wheeler ML, Dunbar SA, Jaacks LM, et al. Macronutrients, food groups, and eating patterns in the management of diabetes: a systematic review of the literature, 2010. Diabetes Care 2012;35:434-45.

68. Crochemore ICC, Souza AFP, de Souza ACF, Rosado EL. v-3 polyunsaturated fatty acid supplementation does not influence body composition, insulin resistance, and 
lipemia in women with type 2 diabetes and obesity. Nutr Clin Pract 2012;27:553-60.

69. Kromhout D, Geleijnse JM, de Goede J, et al. n-3 fatty acids, ventricular arrhythmia-related events, and fatal myocardial infarction in postmyocardial infarction patients with diabetes. Diabetes Care 2011;34:2515-20.

70. Bosch J, Gerstein HC, Dagenais GR, et al. n-3 fatty acids and cardiovascular outcomes in patients with dysglycemia. N Engl J Med 2012;367:309-18.

71. American Diabetes Association. Standards of medical care in diabetes - 2015. Diabetes Care 2015;38:S4.

72. Boulè NG, Haddad E, Kenny GP, et al. Effects of exercise on glycemic controland body mass in type 2 diabetes mellitus: a meta-analysis of controlled clinical trials. JAMA 2001;286:1218-27.

73. Sigal RJ, Kenny GP, Wasserman DH, Castaneda-Sceppa C. Physical activity/exercise and type 2 diabetes. Diabetes Care 2004;27:2518-39.

74. Church TS, Blair SN, Cocreham S, et al. Effects of aerobic and resistance training on hemoglobinA1c levels in patients with type 2 diabetes: a randomized controlled trial. JAMA 2010;304:2253-62.

75. Katzmarzyk PT, Church TS, Craig CL, Bouchard C. Sitting time and mortality from all causes, cardiovascular disease, and cancer. Med Sci Sports Exerc 2009;41:9981005 .

76. Coates A, McGhee H, Slokes H, Thompson D, eds. BACR Guideline for Cardiac Rehabilitation. Oxford: Blackwell Scientific; 1995.

77. US Department for Health and Human Services. Cardiac rehabilitation. Clinical Practice Guideline No 17. AHCPR Publication no. 96-0672. Rockville (MD): Agency for Healthcare Policy and Research; 1995.

78. ATS Committee on Proficiency Standards for Clinical Pulmonary Function Laboratories. ATS statement: guidelines for the six-minute walk test. Am J Respir Crit Care Med 2000;166:111-7.

79. Pollock ML, Wilmore JH. Exercise in health disease: evaluation and prescription and rehabilitation, 2nd ed. Philadelphia: W. B. Sounders Company; 1990.

80. Chu L, Hamilton J, Riddell MC. Clinical management of the physically active patient with type 1 diabetes. Phys Sports Med 2011;39:64-77.

81. Colberg SR. Exercise and diabetes: a clinician's guide to prescribing physical activity, 1st ed. Alexandria, VA: American Diabetes Association; 2013.

82. Pop-Busui R, Evans GW, Gerstein HC, et al. Effects of cardiac autonomic dysfunction on mortality risk in the Actionto Control Cardiovascular Risk in Diabetes (ACCORD) trial. Diabetes Care 2010;33:1578-84.

83. Spallone V, Ziegler D, Freeman R, et al. Cardiovascular autonomic neuropathy in diabetes: clinical impact, assessment, diagnosis, and management. Diabetes Metab Res Rev 2011;27:6392653.

84. Dormandy JA,Charbonnel B, Eckland DJA, et al. Secondary prevention of macrovascular events in patients with type 2 diabetes in the PROactive Study (PROspectivepioglitAzone Clinical Trial In macroVascular Events): a randomised controlled trial. Lancet 2005;366:1279-89.

85. Scirica BM, Bhatt DL, Braunwald E, et al. Saxagliptin and cardiovascular outcomes in patients with type 2 diabetes mellitus. N Engl J Med 2013;369:1317-26.

86. ORIGIN Trial Investigators, Gerstein H, Yusuf S, et al.
Rationale, design, and baseline characteristics for a large international trial of cardiovascular disease prevention in people with dysglycemia: the ORIGIN Trial (Outcome Reduction with an Initial Glargine Intervention). Am Heart J 2008; 155:26-32.

87. Green JB, Bethel MA, Armstrong PW, et al. Effect of sitagliptin on cardiovascular outcomes in type 2 diabetes. N Engl J Med 2015 [Epub ahead of print].

88. Arguedas JA, Leiva V, Wright JM. Blood pressure targets for hypertension in people with diabetes mellitus. Cochrane Database Syst Rev 2013;10:CD008277.

89. McBrien K, Rabi DM, Campbell N, et al. Intensive and standard blood pressure targets in patients with type 2 diabetes mellitus: systematic review and meta-analysis. Arch Intern Med 2012;172:1296-303.

90. ACCORD Study Group; Cushman WC, Evans GW, et al. Effects of intensive blood-pressure control in type 2 diabetes mellitus. N Engl J Med 2010;362:1575-85.

91. Zanchetti A, Grassi G, Mancia G. When should antihypertensive drug treatment be initiated and to what levels should systolic blood pressure be lowered? A critical reappraisal. J Hypertens 2009;27:923-34.

92. American Diabetes Association. Standards of Medical Care for patients with diabetes mellitus. Diabetes Care 2002;25:S33-49.

93. Chobanian AV, Bakris GL, Black HR, et al. National Heart, Lung, Blood Institute; National High Blood Pressure Education Program Coordinating Committee. Seventh report of the Joint National Committee on Prevention, Detection, Evaluation, and Treatment of High Blood Pressure. Hypertension 2003;42:1206-52.

94. Mancia G, De Backer G, Dominiczak A, et al. 2007 Guidelines for the management of arterial hypertension: The Task Force for the Management of Arterial Hypertension of the European Society of Hypertension (ESH) and of the European Society of Cardiology (ESC) Eur Heart J 2007;28:1462-536.

95. Mancia G, Fagard R, Narkiewicz K, et al. 2013 ESH/ESC Guidelines for the management of arterial hypertension. The Task Force for the management of arterial hypertension of the European Society of Hypertension (ESH) and of the European Society of Cardiology (ESC). J Hypertens 2013;31:1281-357.

96. James PA, Oparil S, Carter BL, et al. 2014 evidencebased guideline for the management of high blood pressure in adults: report from the panel members appointed to the Eighth Joint National Committee (JNC 8). JAMA 2014;311:507-20.

97. Patel A; ADVANCE Collaborative Group; MacMahon $\mathrm{S}$, et al. Effects of a fixed combination of perindopril and indapamide on macrovascular and microvascular outcomes in patients with type 2 diabetes mellitus (the ADVANCE trial): a randomised controlled trial. Lancet 2007;370:829-40.

98. Psaty BM, Smith NL, Siscovick DS, et al. Health outcomes associated with antihypertensive therapies used as first-line agents. A systematic review and meta-analysis. JAMA 1997;277:739-45.

99. Bakris GL, Williams M, Dworkin L, et al. Preserving renal function in adults with hypertension and diabetes: a consensus approach. National Kidney Foundation Hypertension and Diabetes Executive Committees Working Group. Am J Kidney Dis 2000;36:646-61. 
100. Collins R, Armitage J, Parish S, et al. MRC/BHF Heart Protection Study of cholesterol lowering with simvastatin in 5963 people with diabetes: a randomised placebo-controlled trial. Lancet 2003;361:2005-16.

101. Shepherd J, Barter P, Carmena R, et al. Effect of lowering LDL cholesterol substantially below currently recommended levels in patients with coronary heart disease and diabetes: the Treating to New Targets (TNT) study. Diabetes Care 2006;29:1220-6.

102. Sever PS, Poulter NR, Dahlöf B, et al. Reduction in cardiovascular events with atorvastatin in 2,532 patients with type 2 diabetes: Anglo-Scandinavian Cardiac Outcomes Trial lipid lowering arm (ASCOT-LLA). Diabetes Care 2005;28:1151-7.

103. Colhoun HM, Betteridge DJ, Durrington PN, et al. Primary prevention of cardiovascular disease with atorvastatin in type 2 diabetes in the Collaborative Atorvastatin Diabetes Study (CARDS): multicentre randomized placebo-controlled trial. Lancet 2004;364:685-96.

104. Kearney PM, Blackwell L, Collins R, et al. Efficacy of cholesterol-lowering therapy in 18,686 people with diabetes in 14 randomised trials of statins: a meta-analysis. Lancet 2008;371:117-25.

105. Taylor F, Huffman MD, Macedo AF, et al. Statins for the primary prevention of cardiovascular disease. Cochrane Database Syst Rev 2013;1:CD004816.

106. Carter AA, Gomes T, Camacho X, et al. Risk of incident diabetes among patients treated with statins: population based study. BMJ 2013;346:f2610.

107. Cannon CP, Braunwald E, McCabe CH, et al. Pravastatin or atorvastatin evaluation and infection therapy-thrombolysis in myocardial infarction 22 investigators. Intensive versus moderate lipid lowering with statins after acute coronary syndromes. N Engl J Med 2004;350: 1495-504.

108. De Lemos JA, Blazing MA, Wiviott SD, et al. Early intensive vs a delayed conservative simvastatin strategy in patients with acute coronary syndromes: phase $\mathrm{Z}$ of the A to Z trial. JAMA 2004;292:1307-16.

109. Nissen SE, Tuzcu EM, Schoenhagen P, et al. Effect of intensive compared with moderate lipid-lowering therapy on progression of coronary atherosclerosis: a randomized controlled trial. JAMA 2004;291:1071-80.
110. Ginsberg HN, Elam MB, Lovato LC, et al. Effects of combination lipid therapy in type 2 diabetes mellitus. N Engl J Med 2010;362:1563-74.

111. Singh IM, Shishehbor MH, Ansell BJ. Highdensity lipoprotein as a therapeutic target: a systematic review. JAMA 2007;298:786-98.

112. Keech A, Simes RJ, Barter P, et al. Effects of long-term fenofibrate therapy on cardiovascular events in 9795 people with type 2 diabetes mellitus (the FIELD study): randomised controlled trial. Lancet 2005;366:1849-61.

113. Boden WE, Probstfield JL, Anderson T, et al. Niacin in patients with low HDL cholesterol levels receiving intensive statin therapy. N Engl J Med 2011;365:2255-67.

114. Baigent C, Blackwell L, Collins R, et al. Aspirin in the primary and secondary prevention of vascular disease: collaborative meta-analysis of individual participant data from randomised trials. Lancet 2009;373:1849-60.

115. Perk J, De Backer G, Gohlke H, et al. European Guidelines on cardiovascular disease prevention in clinical practice (version 2012). The Fifth Joint Task Force of the European Society of Cardiology and Other Societies on Cardiovascular Disease Prevention in Clinical Practice (constituted by representatives of nine societies and by invited experts). Eur Heart J 2012;33:1635-701.

116. Ogawa H, Nakayama M, Morimoto T, et al. Low-dose aspirin for primary prevention of atherosclerotic events in patients with type 2 diabetes: a randomized controlled trial. JAMA 2008;300:2134-41.

117. Belch J, MacCuish A, Campbell I, et al. The prevention of progression of arterial disease and diabetes (POPADAD) trial: factorial randomized placebo controlled trial of aspirin and antioxidants in patients with diabetes and asymptomatic peripheral arterial disease. BMJ 2008;337:a1840.

118. Campbell CL, Smyth S, Montalescot G, Steinhubl SR. Aspirin dose for the prevention of cardiovascular disease: a systematic review. JAMA 2007;297:2018-24.

119. Vandvik PO, Lincoff AM, Gore JM, et al. Primary and secondary prevention of cardiovascular disease: antithrombotic therapy and prevention of thrombosis, 9th ed: American College of Chest Physicians Evidence-Based Clinical Practice Guidelines. Chest 2012;141:e637S$68 \mathrm{~S}$. 


\section{LINEE GUIDA PER GLI AUTORI}

I Quaderni dell 'Italian Journal of Medicine (Quaderni ITJM), costituiscono una collana supplementare solo online annessa alla rivista Italian Journal of Medicine contenente lavori solo in lingua italiana.

I Quaderni ITJM pubblicano:

- Monografie ad hoc individuate dal Presidente FADOI, dal Consiglio Direttivo, dal Board Scientifico o dall'Editor in Chief dell'Italian Journal of Medicine, in funzione del contesto scientifico-istituzionale attuale.

- Monografie ad hoc su temi di particolare rilevanza scientifica a cura della Commissione FADOI Giovani, coordinata dalla Dr.ssa Paola Gnerre.

- Traduzioni in italiano di alcuni lavori pubblicati sui numeri standard dell'Italian Journal of Medicine, di particolare interesse per la comunità scientifica. La selezione è a cura dell'Editor-inChief, Prof. Roberto Nardi.

\section{STESURA DEI LAVORI}

I lavori dovranno essere redatti in modo conforme alle linee guida sotto riportate:

- I manoscritti devono essere scritti interamente in lingua italiana, su documento di Word, con carattere Times New Roman/Arial, dimensione 12, formato $\mathrm{A} 4$, interlinea doppia e margini $2,54 \mathrm{~cm}$. Parole totali: max 4000; Sommario/Abstract: $\max 250$ parole; Bibliografia: $\min 40$ voci; Tabelle e Figure: $3 / 5$ totali (le tabelle non devono superare n. 1 pagina del documento in Word).

- La strutturazione del contenuto deve attenersi agli standard internazionali per la Rassegna (Review): i) Abstract riassuntivo dell'intero lavoro; ii) Introduzione al tema trattato; iii) Criteri e strumenti di ricerca (criteri di inclusione/esclusione, banche dati consultate, ...); iv) i successivi paragrafi devono illustrare le più recenti scoperte scientifiche nel settore; v) Conclusioni; vi) Bibliografia.

- La prima pagina deve riportare: i) titolo (in stampatello minuscolo), senza acronimi; ii) nome e cognome per esteso di ciascun autore; iii) affiliazione(i) di ciascun autore, numerate con numeri arabi; iv) eventuali ringraziamenti; v) nome e indirizzo postale completi dell'autore corrispondente, corredati da telefono, fax, e-mail; vi) da 3 a 5 parole chiave, separate da virgola. La seconda pagina può riportare: i) contributi degli autori, e.g. informazioni relative a contributi sostanziali delle persone coinvolte nello studio (http://www.icmje.org/\#author); ii) dichiarazione relativa a potenziali conflitti d'interesse; iii) ulteriori informazioni (e.g. fondi, esposizioni durante conferenze...).

- In caso di utilizzo di tabelle, queste devono essere tutte numerate con numeri arabi e citate nel testo in ordine consecutivo (e.g. NON nominare le tabelle come Tabella $1 \mathrm{~A}, 1 \mathrm{~B}, \ldots$ o $1.0,1.1, \ldots)$. Le tabelle devono essere presentate in formato editabile. Ciascuna tabella deve essere corredata da una breve didascalia; in caso di abbreviazioni, riportare una nota a piè di CIASCUNA tabella che spieghi TUTTE le abbreviazioni presenti in ognuna.

- In caso di utilizzo di figure, queste devono essere inviate in formato .tiff o .jpg, allegate al manoscritto in singoli files, secondo le seguenti specifiche:

i) a colori (salvate in modalità CMYK): minimo 300 dpi di risoluzione;

ii) in bianco e nero: minimo $600 \mathrm{dpi}$ di risoluzione;

iii) minimo $17,5 \mathrm{~cm}$ di larghezza.

Ciascuna figura deve essere corredata da una breve didascalia.

NB: In caso di Tabelle/Figure riprese e/o modificate da altri lavori già pubblicati, sarà cura degli autori accertarsi se tali materiali siano o meno coperti da copyright e procurarsi i permessi necessari per la riproduzione. Tali permessi dovranno essere allegati alla versione definitiva del lavoro. L'ufficio editoriale si riserva la facoltà di rimuovere Tabelle/Figure coperte da copyright, se sprovviste dei necessari permessi.

- In caso di utilizzo di abbreviazioni, la prima volta che esse sono citate è necessario scrivere per esteso la definizione+abbreviazione tra parentesi tonde [e.g. risonanza magnetica $(\mathrm{RMN})]$, a seguire si dovrà riportare solo l'abbreviazione (unica eccezione: nei titoli e nelle didascalie di tabelle e figure NON si utilizzano abbreviazioni).

\section{BIBLIOGRAFIA}

Le voci bibliografiche devono essere formattate secondo lo stile Vancouver.

Nella sezione Bibliografia, le voci bibliografiche devono essere numerate consecutivamente nell'ordine in cui appaiono per la prima volta nel testo (NON in ordine alfabetico) e, nel testo, devono essere indicate con numeri arabi in apice. Voci bibliografiche riferite a comunicazioni personali o dati non pubblicati devono essere incorporate nel testo e NON inserite tra le voci numerate [e.g. (Wright 2011, dati non pubblicati) o (Wright 2011, comunicazione personale)]. Le voci bibliografiche nella sezione Bibliografia devono tassativamente essere preparate come segue:

i) più di 3 autori, citare 3 autori, et al. Se il lavoro contiene solo 4 autori, citarli tutti e 4 ;

ii) titolo del lavoro in stampatello minuscolo;

iii) nome della rivista, senza punti, abbreviato secondo gli standard internazionali; in caso di dubbi sulla corretta abbreviazione, fare riferimento ai seguenti siti:

a. ISI Journal Abbreviations Index (http://library.caltech.edu/ reference/abbreviations/);

b. Biological Journals and Abbreviations (http://home.ncifcrf. gov/research/bja/);

c. Medline List of Journal Titles (ftp://ftp.ncbi.nih.gov/pubmed/J_Medline.txt);

iv) inserire l'anno di pubblicazione subito dopo il nome della rivista, seguito da punto e virgola;

v) NON inserire giorno o mese di pubblicazione;

vi) citare solo il volume, seguito dai due punti (NON citare il fascicolo tra parentesi);

vii) abbreviare le pagine, e.g. 351-8.

Per accertarsi di aver correttamente formattato le voci bibliografiche, confrontarle con le citazioni in PubMed (http://www.ncbi. nlm.nih.gov/pubmed).

Esempi (prestare attenzione anche alla punteggiatura):

Articolo standard su Rivista

Halpern SD, Ubel PA, Caplan AL. Solid-organ transplantation in HIV-infected patients. N Engl J Med 2002;347:284-7.

\section{Proceedings}

Christensen S, Oppacher F. An analysis of Koza's computational effort statistic for genetic programming. In: Foster JA, Lutton E, Miller J, Ryan C, Tettamanzi AG, eds. Genetic programming. EuroGP 2002: Proceedings of the 5th European Conference on Genetic Programming, 2002 Apr 3-5, Kinsdale, Ireland. Berlin: Springer; 2002. pp 182-91.

Articoli i cui autori sono Organizzazioni

Diabetes Prevention Program Research Group. Hypertension, insulin, and proinsulin in participants with impaired glucose tolerance. Hypertension 2002;40:679-86.

Libri

Murray PR, Rosenthal KS, Kobayashi GS, Pfaller MA. Medical microbiology. 4th ed. St. Louis, MO: Mosby; 2002. (CITAZIONE DEL LIBRO INTERO)

Meltzer PS, Kallioniemi A, Trent JM. Chromosome alterations in human solid tumors. In: Vogelstein B, Kinzler KW, eds. The genetic basis of human cancer. New York, NY: McGraw-Hill; 2002. pp 93113. (CITAZIONE DI UN CAPITOLO)

\section{MODALITÀ D'INVIO DEI LAVORI}

\section{Monografie}

Gli Autori dovranno fare riferimento all'Editor-in-Chief o alle persone da lui designate nelle lettere di invito a scrivere gli articoli programmati.

Per gli articoli inviati da giovani internisti, gli Autori dovranno fare riferimento alla Dr.ssa Paola Gnerre (e-mail: pgnerre@yahoo.it)e agli eventuali altri referenti da lei designati, nelle prime fasi di stesura dei manoscritti, revisioni e correzioni. 


\section{QUADERNI}

La Dr.ssa Gnerre raccoglierà poi le versioni definitive dei lavori di ciascuna monografia e provvederà all'invio di tutti i materiali all'ufficio editoriale.

I lavori solo nella loro versione definitiva e approvata dalla Commissione FADOI Giovani dovranno pervenire all'ufficio editoriale già pronti per l'impaginazione e immediata pubblicazione (già corredati da eventuali permessi per la riproduzione di tabelle e immagini).

\section{Traduzioni}

Previo invito dell'Editor-in-Chief, gli Autori dovranno far pervenire all'ufficio editoriale la versione tradotta in italiano, al seguente indirizzo e-mail: paola.granata@pagepress.org

Il file in formato Word dovrà essere formattato secondo gli standard editoriali della rivista ufficiale ed essere già pronto per impagina- zione e immediata pubblicazione (corredato da eventuali permessi per la riproduzione di tabelle e immagini).

Si prega di inviare le eventuali tabelle in formato editabile e le figure in alta definizione secondo gli standard sopra riportati.

\section{NOTA PER GLI AUTORI}

I lavori pubblicati sui Quaderni ITJM non verranno indicizzati, ma saranno liberamente disponibili in un'apposita sezione del sito FADOI (http://www.fadoi.org/).

Gli Autori i cui lavori siano accettati per la pubblicazione sui Quaderni ITJM e che fossero interessati a vederli pubblicati anche sulla rivista ufficiale, dovranno sottomettere attraverso il sito dell'ITJM (www.italjmed.org) la versione (già tradotta) in inglese e redatta in modo conforme alle linee guida della rivista; seguiranno poi la procedura di selezione tramite peer review e, se accettati, saranno inseriti nel piano editoriale standard.

\section{STAFF EDITORIALE}

Paola Granata, Journal Manager

paola.granata@pagepress.org

Selvaggia Stefanelli, Marketing Manager

marketing@pagepress.org

Claudia Castellano, Production Editor

Tiziano Taccini, Technical Support

\section{QUADERNI - ITALIAN JOURNAL OF MEDICINE}

Tutti gli articoli pubblicati sui QUADERNI - Italian Journal of Medicine sono redatti sotto la responsabilità degli Autori. La pubblicazione o la ristampa degli articoli della rivista deve essere autorizzata per iscritto dall'editore. Ai sensi dell'art. 13 del D.Lgs 196/03, i dati di tutti i lettori saranno trattati sia manualmente, sia con strumenti informatici e saranno utilizzati per l'invio di questa e di altre pubblicazioni e di materiale informativo e promozionale. Le modalità di trattamento saranno conformi a quanto previsto dall'art. 11 del D.Lgs 196/03. I dati potranno essere comunicati a soggetti con i quali PAGEPress intrattiene rapporti contrattuali necessari per l'invio delle copie della rivista. Il titolare del trattamento dei dati è PAGEPress Srl, via Belli 7 - 27100 Pavia, al quale il lettore si potrà rivolgere per chiedere l'aggiornamento, l'integrazione, la cancellazione e ogni altra operazione di cui all'art. 7 del D.Lgs 196/03.

Editore: PAGEPress srl, via G. Belli 7, 27100 Pavia, Italy www.pagepress.org
PUBBLICATO DA

PAGEPress Publications

via G. Belli 7

27100 Pavia, Italy

T. +39.0382 .1751762$

F: +39.0382 .1750481$

\section{pagepress}

www.pagepress.org

info@pagepress.org
Pubblicato: novembre 2015. 
PAGEPress - Scientific Publications

Via G. Belli, 7 - 27100 Pavia - Italy

Phone +390382 1751762 - Fax +390382 1750481

www.pagepress.org 